\title{
Affektpoetik der \\ Forschungsmemoiren
}

\section{1 Übermenschen und Menschenaffen: Edgar Rice Burroughs' Tarzan of the Apes (1912/14)}

\begin{abstract}
Ihr habt den Weg vom Wurme zum Menschen gemacht, und Vieles ist in euch noch Wurm. Einst wart ihr Affen, und auch jetzt noch ist der Mensch mehr Affe, als irgendein Affe. ${ }^{1}$
\end{abstract}

\subsubsection{Irritation zwischen Affekt und Text}

Im Vorangegangenen ging es anhand der primatologischen Forschungsmemoiren um die Darstellung einer neuen Form der Forschung im Feld, die nicht nur auf der Partizipation von Emotionalität an epistemologischen Vorgängen, sondern auf der Methode einer teilnehmenden und empathischen Beobachtung beruht. Im Sinne von Breithaupts bereits angeführter Empathie-Theorie ist dies eine über eine primär kognitive Perspektivübernahme in der ,Theory of Mind“ hinausgehende Form der Beobachtung, die nicht nur das „Verhalten und die Emotionen eines anderen [registriert], ,als wäre er selbst der Handelnde “", 2 sondern auch eine Parteinahme ohne notwendige Handlungsintention bedeutet. ${ }^{3}$ Jane Goodall selbst verteidigt die Methode in der ausführlichsten und zugleich formal ,wissenschaftlichsten' Darstellung ihrer langjährigen Forschungsarbeit, The Chimpanzees of Gombe, wie folgt:

\footnotetext{
${ }^{1}$ Nietzsche, Friedrich: Also sprach Zarathustra. Hg. von Giorgio Colli und Mazzino Montinari. München 1999 (Kritische Studienausgabe Bd. 4), S. 14.

${ }^{2}$ Breithaupt: Kulturen der Empathie, S. 152.

${ }^{3}$ Vgl. Breithaupt: Kulturen der Empathie, S. 152 ff.
} 
$[\mathrm{M}]$ any of us who have worked at Gombe over the years have developed a degree of empathy with the individuals studied. In itself, this is not a bad thing. Subtle communication cues denoting slight changes in ,mood ' or attitude toward other chimpanzees are more readily detected once empathy of this kind has been established and this can aid us in the understanding of complex social processes. [...] Intuitive interpretations, which may be based on an understanding stemming directly from empathy with the subject, can be tested afterwards against the facts set out in the data. ${ }^{4}$

Emotionalität wird also, so ließ sich anhand einzelner Szenen darlegen, von den Autor/innen als Teil ihrer Methodik kultiviert und nutzbar gemacht - durchaus im Einklang mit tradierten Formen der epistemologisch und intersubjektiv nutzbaren Affektivität.

Im nun folgenden Teil soll der Blick auf das ,Andere" einer solchen emotionalen Öffnung in der Feldforschungsarbeit gerichtet werden. Die Annäherung an das Forschungstier und die Erschließung einer Forschungs- als Beobachtungs- und Partizipationsbeziehung wird in den Forschungsmemoiren eindrücklich in Szenen der Begegnung zugespitzt. Hierbei wird eine Emotionsarbeit aufgenommen und beschrieben, die sich einem ,positiven' affektiven Spektrum zuordnen lässt. Die affektive Sondierung des epistemischen Gegenstandes in der primatologischen Feldforschung führt lange Zeit zu relativ stabilen Forschungsbeziehungen und -situationen. In diesen lassen sich jedoch, das schildern alle Autor/innen gleichermaßen in den hier behandelten Texten, mit der Zeit zunehmend neue Verhaltensweisen oder Verhaltensänderungen beobachten, die affektlogisch als problematisch aufgefasst werden: ${ }^{5}$ Gerade dort, wo in den 1950er bis 1970er Jahren die Menschenaffen als Modell einer relativ friedfertigen und ,einfachen" menschlichen Frühform konzipiert und beobachtet wurden, führen neue Erkenntnisse über das Sexualverhalten oder gewalttätige

\footnotetext{
${ }^{4}$ Goodall, Jane: The Chimpanzees of Gombe. Patterns of Behavior. Cambridge, Mass./London 1986, S. 58. Im Folgenden, wenn zur Unterscheidung nötig, als $C G$ geführt; Seitenangaben im Text. Zu einer normativen Vorstellung von Empathie als ethischer Praxis im Umgang mit Tieren siehe auch Gruen, Lori: Sich Tieren zuwenden: Empathischer Umgang mit der mehr als menschlichen Welt. In: Schmitz, Friederike (Hg.): Tierethik. Grundlagentexte. Berlin 2014, S. 390-404.

${ }^{5}$ Der Begriff ,Affektlogik' geht auf den Schweizer Psychiater Luc Ciompi zurück. Das Ausgangspostulat Ciompis betrifft die untrennbare Verbindung und gesetzmäßige Zusammenarbeit emotionaler und kognitiver Komponenten in sämtlichen psychischen Leistungen. Vgl. Ciompi: Die emotionalen Grundlagen des Denkens, S. 46. In der Übertragung auf soziale Prozesse hat Elke Endert danach gefragt, auf welche Weise Emotionen nicht nur die Wahrnehmung und das Denken beeinflussen, sondern auch das Handeln bedingen, unter der Voraussetzung, dass logische Verknüpfungen affektgeleitet sind. Vgl. Endert, Elke: Über die emotionale Dimension sozialer Prozesse. Die Theorie der Affektlogik am Beispiel der Rechtsextremismus- und Nationalsozialismusforschung. Konstanz 2006, S. 13, 37. Affektlogiken sollen im Folgenden mit Endert verstanden werden als individuelle, durch Erfahrung und soziale Interaktion entstandene, affektiv geprägte und eingeübte Denk- und Wissenssysteme, die das Handeln und Verhalten bestimmen. Affektlogisch problematisch ist folglich etwas, das als außerordentlich (bzw. unsystematisch) wahrgenommen wird, darin gegen die eingeübten und tradierten affektiven Wissensbestände und individuellen Verhaltensregeln antritt und daher auch auf affektiver Ebene irritierend ist.
} 
Auseinandersetzungen $\mathrm{zu}$ so bahnbrechenden wie für das forschende Subjekt verstörenden neuen Erkenntnissen. Wie Jane Goodall 1986 rückblickend schreibt:

Had my colleagues and I stopped after a mere ten years, we should have had a very different picture of the Gombe chimpanzees than we do today. We would have observed many similarities in their behaviour and ours, but we would have been left with the impression that chimpanzees were far more peaceful than humans. $(C G, 3)$

Ich möchte in diesem Zusammenhang zunächst von affektiven Irritationsmomenten sprechen, die sich angesichts verschiedener Szenarien für die Verhaltensforscher/innen auftun - dort wo die eingeübte Affektlogik dieser Form der Feldforschung auf neue Sachverhalte stößt, die affektiv herausfordernd sind. ,Irritation" lässt sich dabei in einem ebenso mehrschichtigen Sinn verwenden, wie ihn die etymologische Herleitung erlaubt: Als eine Form der (physisch-materiellen) Störung (irritare), ,to vex or provoke, but also to inflame and physically irritate“, 6 wie Metelmann und Loren dies als ästhetisch-ethisches Phänomen definieren, und als affektives Phänomen, wie Sianne Ngai dies u. a. in Bezug auf Aristoteles umreißt als ein ,,minor [...] dysphoric affect". ${ }^{7}$ Metelmann und Loren weisen in ihrer Analyse zudem auf die lateinische Wurzel irratus hin, aus der heraus sich Irritation auch als Hinweis darauf verstehen lässt, ,that something is invalid or void“. 8 Eine Ästhetik der Irritation, wie sie die beiden Autoren konzipieren, lässt dramatisch-affektive Versenkung und eine Entfremdung durch ästhetisch-narrative Formen aufeinandertreffen. $^{9}$

Eine solche Ästhetik der Irritation lässt sich m. E. in Teilen bereits in einem frühen Text finden, der von einem Menschen unter Affen handelt: Edgar Rice Burroughs Tarzan of the Apes ${ }^{10}$ (1912/14). Der erstaunlich erfolgreiche Tarzan-Stoff hat besonders in der originären literarischen Roman-Form von Burroughs ${ }^{11}$ und in

${ }^{6}$ Loren, Scott/Metelmann, Jörg: Irritation of Life. The Subversive Melodrama of Michael Haneke, David Lynch and Lars von Trier. Marburg 2013, S. 14.

${ }^{7}$ Ngai: Ugly Feelings, S. 181. Ngai konzeptualisiert Irritation als eine Stimmung im und am Text, die indexikalisch auf einen (emotionalen) Missstand hinweist und als affektives Text-Phänomen meist in Verbindung mit einer inadäquaten Äußerung von Wut über ein Unrecht steht. Vgl. S. 175.

${ }^{8}$ Loren/Metelmann: Irritation of Life, S. 14.

${ }^{9}$, ,T] $\mathrm{The}$ aesthetic of irritation we outline in what follows sets affective dramatic engulfment and defamiliarizing aesthetico-narrative forms in an anatagonistic but densely interdependent bind with one another". Loren/Metelmann: Irritation of Life, S. 21.

${ }^{10}$ Burroughs, Edgar Rice: Tarzan of the Apes [1914]. Hg. von John Haslam. Oxford 2010. Im Folgenden, wenn zur Unterscheidung nötig, als TA geführt; Seitenangaben im Text.

${ }^{11}$ Burroughs veröffentlichte Tarzan of the Apes zunächst im Oktober 1912 im All-Story Magazine. Zwei Jahre später erschien der Text mit Änderungen in Buchform bei McClurg. Zwischen 1914 und 1967 erschienen 25 weitere Bände. 
den Filmen mit Johnny Weissmüller ${ }^{12}$ bereits vielfach auch wissenschaftliche Aufmerksamkeit erfahren. Dies mag daran liegen, dass Burroughs' Werk mit Edward Said gesprochen als ,an unimaginable, totally unlikely hodgepodge of polymorphous perversity"13 viele Ansatzpunkte für eine kulturwissenschaftliche Analyse bietet. Dabei stand im Mittelpunkt vor allem der Roman als Kolonial-, Bildungsund Evolutionsroman, Tarzan als Grenz- und (prä-)ödipale Figur, als amerikanisches Männlichkeitsideal und sogar als Immigrant. ${ }^{14}$ Auch die Rolle von Medien und Frauen (oder Frauen als Medien der Zivilisierung) wurde untersucht. ${ }^{15}$ Bislang wenig Beachtung hat dabei der affektive Gehalt dieses Stoffes über den von Affen aufgezogenen, sich primatisch sozusagen selbst evoluierenden englischen Lord gefunden. Dabei scheint besonders der erste Burroughs-Roman Tarzan of the Apes dank der von Said bescheinigten , hodgepodge'-Artigkeit ein irritierendes Spiel mit der Affektlogik zumindest seines postmodernen Publikums zu spielen.

\subsubsection{Ich Mensch, Du Affe? Tarzan und die Affen}

Edgar Rice Burroughs' Tarzan of the Apes erzählt von einem zufälligen Aufzuchtexperiment und nutzt Affen, um die Vorzüglichkeit des menschlichen Protagonisten zu betonen. Burroughs’ erster Tarzan-Roman lässt sich daher durchaus mit Blick auf seinen primatologischen Gehalt interpretieren. Ihm ist die Tradition der Auseinandersetzung mit der Verwandtschaft von Mensch und Affe ebenso anzumerken wie die Kenntnis der zeitgenössischen Forschung am Affen. In seiner Einführung hat Jason Haslam bereits darauf hingewiesen, dass Burroughs zwar vor allem durch ,popular descriptions from exploration narratives“16 wie Paul Du

\footnotetext{
${ }^{12}$ Die ersten beiden Verfilmungen des Stoffes sind Stummfilme aus dem Jahr 1918. Ab 1932 spielte Weissmüller Tarzan in elf Tonfilmen und führte die Figur zu enormer Bekanntheit. Die anhaltende Popularität zeigt sich an den neueren Verfilmungen: Hudson, Hugh: Greystoke: The Legend of Tarzan, Lord of the Apes. Warner Bros. 1984; Lima, Kevin/Buck, Chris: Tarzan. Walt Disney Pictures/Buena Vista Pictures 1999; Yates, David: The Legend of Tarzan. Warner Bros. Pictures 2016.

${ }^{13}$ Said, Edward: Jungle Calling. On Johnny Weissmuller's Tarzan. In: Ders.: Reflections on Exile and Other Essays. Cambridge, Mass. 2000, S. 327-336, hier S. 329.

${ }^{14}$ Vgl. Krüger/Mayer/Sommer: Figuren des Dazwischen; Richter: Literature after Darwin, S. 80 ff.; Bederman, Gail: Manliness and Civilization: A Cultural History of Gender and Race in the United States, 1880-1917. Chicago u. a. 1995, S. 221 f.; Griem: Monkey Business, S. 167 f.; Said: Jungle Calling.

${ }^{15}$ Siehe Krüger, Gesine: Schrift und Gewalt bei Tarzan. Meuchelmorde - Liebesbriefe - (unmögliche) Utopien. In: Gesine Krüger/Ruth Mayer/Marianne Sommer (Hg.): „Ich Tarzan. “ Affenmenschen und Menschenaffen zwischen Science und Fiction. Bielefeld 2008, S. 21-47; Hediger: Was will Jane im Dschungel?

${ }^{16}$ Haslam, John: Introduction. In: Edgar Rice Burroughs: Tarzan of the Apes. Hg. von John Haslam. Oxford 2010, S. vi-xxvii, hier S. xix.
} 
Chaillus Explorations and Adventures inspiriert war, sich jedoch die Differenzierung von Affe und Mensch bei Burroughs auf frühe ethologische Beschreibungen wie jene des Völkerkundlers und Naturforschers Robert Hartmann in Die menschenähnlichen Affen und ihre Organisation im Vergleich zur menschlichen (1883) beziehen lasse. ${ }^{17}$ Es ist auffällig, wie viel Wert Burroughs in Tarzan of the Apes darauf legt, seine Affen physisch und behavioral realistisch zu gestalten: Der Affe steht hier, ,in a semi-erect position and then placing its backs of its closed fists upon the ground“ (25). Die Fortbewegung wird folgendermaßen beschrieben: „when they walked it was with a rolling, awkward motion, placing the knuckles of their closed hands upon the ground and swinging their ungainly bodies forward“ (31); ,,[u]sing his long arms as a man uses crutches, and rolling his huge carcass from side to side with each stride" (33). Auch die brachiale Fortbewegung findet Erwähnung: „But when the way was through the lower trees they moved swiftly, swinging from branch to branch with the agility of their small cousins, the monkeys“ (31). ${ }^{18}$ Sie äußern ,,deep guttural growls and an occasional low barking sound“ (24). Auch dass der Affennachwuchs dem Menschenkind in den ersten Jahren in der Entwicklung weit überlegen (,far advanced“, 36) ist, wird festgehalten. Die Sozialstruktur des „tribe of anthropoids“ wird, einem patriarchalen Ideal folgend, von ,some six or eight families, each family consisting of an adult male with his wives and their young“" (30) gebildet. ${ }^{19}$

Das Verhalten der männlichen Affen ist bei Burroughs deutlich von Gewalt geprägt: „With a wild scream he was upon her, tearing a great piece from her side with his mighty teeth, and striking her visciously upon her head and shoulders with a broken tree limb until her skull was crushed to jelly“ (29). Verantwortlich dafür sind aufbrausende Emotionen: „,hatred“ (25); ,on a rampage of rage“, ,,in one of his fits of uncontrollable anger", ,infuriated brute“, „the mighty male's temper“, "his fits of demoniacal rage“ (29); ,,in the insanity of his fury“ (86); „his madness“ (89); ,foaming with rage and hatred“, „nursing his spite“, „his pent anger“ (150). Die mangelnde Beherrschung von Emotionen gesellt sich zu einer genauen physischen Beschreibung, die häufig einer physiognomischen

\footnotetext{
${ }^{17}$ Haslam, John: Introduction, S. xx. Im Folgenden wird die englische Übersetzung Hartmanns von 1886 verwendet. Hartmann bezieht sich seinerseits auf die Berichte von Afrika- und Forschungsreisenden. So referiert er beispielsweise ausgiebig auf Paul Du Chaillu, Hugo von Koppenfels und Alfred Russel Wallace. Siehe Hartmann, Robert: Anthropoid Apes. New York 1886 (dt. 1883), S. $241 \mathrm{f}$.

${ }^{18}$ Bei Hartmann heißt es über Gorillas: „In walking they place the backs of their closed fingers on the ground“; "the body, which is never in an upright position as in man, bent forward, rolls to some extent from one side to another“; „the gorilla also throws his arms forward, sets his hands upon the ground, then gives a half-swinging, half-springing motion to his body“. Hartmann: Anthropoid Apes, S. $231 \mathrm{f}$.

${ }^{19}$ Vgl. Hartmann: Anthropoid Apes, S. 237: „The chimpanzee either lives in separate families or in small groups of families".
} 
(Ab-)Wertung dient: ${ }^{20}$ Die Affen haben ,close set, wicked eyes, gleaming beneath shaggy brows“ (19), und ,great canine fangs [...] bared in a horrid snarl“ (25); ihre Stirnen sind „extremely low and receding“ (30). Zudem haben sie eine „flat nose“" und ihre Ohren sind ,large and thin“ (30).

Die Affen, unter denen Tarzan aufwächst, werden jedoch an keiner Stelle als Spezies identifiziert. Obwohl explizit von „your genus“ (206) die Rede ist, wenn der Franzose D’Arnot Tarzan auf die menschliche Vorzüglichkeit hinweist, werden die Affen zwar differenziert, bleiben aber unklassifiziert. Sie sind schlicht „great anthropoid ape[s]“ (24) und verschieden von ,their smaller cousins, the monkeys“ (33). Besonders in der Beschreibung von Tarzans äffischer Ziehmutter Kala wird diese Differenzierung mit der Verweigerung der taxonomischen Klassifikation deutlich. Sie wird vom Gorilla abgegrenzt, aber nicht als Art benannt: Trotz ihrer ungewöhnlich großen „,capacity for mother love and mother sorrow“ ist sie „still an ape, a huge, fierce, terrible beast of a species closely allied to the gorilla, yet more intelligent; which, with the strength of their cousin, made her kind the most fearsome of those awe-inspiring progenitors of man" (30, Hervorheb. MS). ${ }^{21}$ Man macht es sich zu einfach, wenn man dies, wie John Taliaferro in seiner Burroughs-Biografie, schlicht mit den Wissenslücken des Autors begründet. ${ }^{22}$ Denn diese taxonomische Uneindeutigkeit verleiht Burroughs die Lizenz, die unbenannte Art mit allerlei Eigenschaften auszustatten, die aus den Affen eine Ethnie machen: Die Affen haben ein Gemeinschaftsgefühl, das sich vor allem gegenüber Außenseitern bemerkbar macht; sie sprechen eine eigene Sprache (36), kennen Rituale (,the queer rites“, 53), Musik (,those strange earthen drums“ 53; ,the fierce, mad, intoxicating revel of the Dum-Dum") und Tanz (,the mad whirl of the Death Dance“, 56). In der Kombination ergibt dies bei Burroughs eine Zeremonie: „The rites of the Dum-Dum marked important events in the life of the tribe - a victory, a capture of a prisoner, the killing of some large fierce denizen of the jungle, the death or accession of a king, and were conducted with set ceremonialism“ (54). Die Affen haben darüber hinaus eine eigene Rechtsvorstellung (,the inalienable rights of the jungle folk“, 36) und praktizieren sogar rituellen Kannibalismus (56 f.).

\footnotetext{
${ }^{20}$ Dass in diesem Roman ganz besonders Wert darauf gelegt wird, Charakter qua Physis zu markieren, zeigt sich nicht nur an der rassistischen Attribuierung der schwarzen und der sozial unterprivilegierten Figuren, sondern auch daran, dass Tarzans durchweg positiv konnotierte äffische Ziehmutter Kala ,,a splendid, clean-limbed animal, with a round, high forehead, which denoted more intelligence than most of her kind possessed" (TA, S. 30) ist.

${ }^{21}$ Hartmanns Anthropoid Apes listet 1886 unter den ,Anthropoid Apes“ bereits „Gorilla, Chimpanzee, Orang, and Gibbon“ (vgl. S. 290 ff.) auf. Während frühere Tarzan-Verfilmungen die Affen meist entweder mit Schimpansen oder Gorillas besetzten (eine Ausnahme bildet Derek, John: Tarzan, the Ape Man. United Artists/Metro-Goldwyn-Mayer 1981, die einen Orang-Utan einsetzt), nutzt die jüngste filmische Tarzan-Interpretation The Legend of Tarzan die neuen Computertechnologien, um mittels CGI einen Hybrid zu fabrizieren: Ein Gorillakörper mit einem Schimpansengesicht. Allerdings spielen die Affen in dieser Verfilmung eine untergeordnete Rolle.

${ }^{22}$ Siehe Taliaferro, John: Tarzan Forever. The Life of Edgar Rice Burroughs, Creator of Tarzan. New York 1999, S. 76.
} 
Burroughs' Blick in die wissenschaftliche Fachliteratur lässt sich zudem daran erkennen, dass die Formulierungen der eingesprengten zoologischen, ethologischen und ethnologischen Informationen in ihren Generalisierungen ein professionelles Verständnis suggerieren: „A furious leap [...] - a perilous chance which apes seldom if ever take, unless so closely pursued by danger that there is no alternative" (29, Hervorheb. MS); „She did not run; but, after the manner of her kind when not aroused, sought rather to avoid than to escape" (67, Hervorheb. MS). Zum Ritual heißt es geradezu abhandlungsartig: „From this primitive function [the Dum-Dum] has arisen, unquestionably, all the forms and ceremonials of modern church and state" (54). Die ethologischen Informationen und ihre Darstellung gewinnen von derartigen stilistischen Anklängen an wissenschaftliche Äußerungsweisen ihre Autorität und grundieren die Geschichte Tarzans unter bzw. „of the Apes“.

Tarzan wird als Säugling von einer Äffin vor dem Tod gerettet und wächst unter den Affen ihrer Gruppe (hier konsequent: „tribe“) auf. Doch sein Verhältnis zu den Affen ist ein affektiv schwieriges. Zwar schildert uns die Erzählinstanz Tarzans Kindheit ,among these fierce apes“ als „happy“ (37). Doch, wie es in Tarzan heißt: „Kala, alone, he was glad to have with him“ (48). Seine Affenmutter Kala verdient sich mit ihrer „unselfish and sacrificing devotion“ (48) für das Menschenjunge einen Platz in dessen Herz und Achtung. Sobald Tarzan mit zehn Jahren die anthropologische Differenz erkennt - „He was nearly ten before he commenced to realize that a great difference existed between himself and his fellows" (37) - treibt ihn jedoch ein so affektiver wie kognitiver Vektor fort aus dem äffischen Mikrokosmos des Dschungels.

Ein erster Schritt hierzu ist die Selbst-Beobachtung, die bezeichnenderweise in einer anti-narzisstischen Spiegelszene kulminiert. ${ }^{23}$ Tarzan nimmt seinen Körper als „entirely hairless, like some low snake, or reptilian“ (37) wahr. Der Blick in die spiegelnde Oberfläche eines kleinen Sees lässt ihn dann erstmals sein eigenes Gesicht sehen (das er auch sogleich als solches erkennt). Der Erzähler setzt den Auftakt für die Passage mit der Gegenübersetzung von ,[t]he fierce and terrible features of the ape beside those of the aristocratic scion of an old English house" (38). Doch Tarzan selbst sieht hier ganz im Gegenteil:

[T]hat tiny slit of a mouth and those puny white teeth! [...] the little pinched nose of him; so thin was it that it looked half starved. [...] But when he saw his own eyes; ah, that was the final blow - a brown spot, a gray circle and then blank whiteness! Frightful! not even the snakes had such hideous eyes as he. (38)

Tarzans ,personal appraisement of his features“ (38) vis-à-vis der äffischen Norm, mit der er aufgewachsen ist, verursacht ihm durchweg negative Gefühle aus dem Schamspektrum: „feelings of intense shame“ (37), „Tarzan was appalled“, „[h]e turned red“ (38), kurz: „It had been bad enough to be hairless, but to own such

\footnotetext{
${ }^{23} \mathrm{Vgl}$. zu dieser „intriguing inversion of the Lacanian mirror scene“ Richter: Literature after Darwin, S. 82 f.
} 
a countenance! He wondered that the other apes could look at him at all" (38). Wie diese letzte Formulierung zeigt, nimmt sich Tarzan hier selbst noch als Affe unter Affen wahr. In dieser kurzen Phase der Selbst-Wahrnehmung ist es für Tarzan schambehaftet, anders auszusehen als die anderen, d. h. der ästhetischen, integrativen Norm nicht genügen zu können. Hierin zeigt sich jene Scham, wie sie die neuere Forschung konzipiert:

In shame, we take it that we exemplify a specific disvalue that strikes us as an indication of our incapacity to exemplify a self-relevant value even to a minimal degree. This experience $[\ldots]$ affects the self in a distinctive way. Our identity being constituted by the values to which we are attached, it is shaken precisely insofar as we experience our inability to honor even minimally the demands that go with this value. ${ }^{24}$

Dieses Anderssein schlägt in Tarzan jedoch rasch um in eine Auszeichnung: Uns wird anschließend an diese Spiegelszene eine Situation der Gefahr vorgeführt der Angriff durch eine Löwin -, in der Tarzan seine „,self-confidence“, „higher intelligence“, „quickness of mental action far beyond the powers of the apes" und „resourcefulness which were the badges of his superior being“ (alles 39) entdeckt. Lag zuvor schon ein zwar der Introspektion zugeneigter, aber doch distanzierter Blick auf den Affen, erscheinen sie fortan nur noch als Folie, vor der sich Tarzan als Mensch beweisen kann. Von nun an driftet Tarzan fort von den Affen und wird zum Einzelgänger, der durch Beobachtung und Experiment erkennt und lernt und seine Fertig- und Fähigkeiten ausbaut. Dies hilft ihm zunächst in einer geradezu hegelianischen Szene der Anerkennungsgewinnung bei der Unterwerfung des äffischen Gegners, ${ }^{25}$ lässt ihn seine rass(ist)ische Überlegenheit über Afrikaner erkennen und macht ihn schließlich, zum Ende des Romans, zum bis zur distinguierten Melancholie hin vollendeten Gentleman.

\subsubsection{Tarzan of the Apes als primatologische Studie}

Beobachtung ist ein zentraler Darstellungs- und Erzählmodus des Romans. John und Alice Clayton, Lord und Lady Greystoke, betrachten als Zeugen die Meuterei an Bord des sie transportierenden Schiffes (,The sight which met his eyes confirmed his worst fears“, 14) und die Abreise der Meuterer von der Küste (,Clayton and his wife stood silently watching their departure“, 18). Sie selbst sind jedoch bereits Objekt einer anderen Beobachtung: „And behind them, over the edge

\footnotetext{
${ }^{24}$ Deonna, Julien A./Rodogno, Raffaele/Teroni, Fabrice: In Defense of Shame. The Faces of an Emotion. Oxford/New York 2011, S. 122.

${ }^{25}$ Als Tarzan im ,Endkampf‘ mit seinem Adoptivbruder Terkoz dessen Nacken brechen könnte, reflektiert er mit ,,a man's reasoning power“: ,If I kill him [...] what advantage will it be to me? [...] [I]f Terkoz be dead, he will know nothing of my supremacy, while alive he will ever be an example to the other apes“ (TA, S. 93). Zur anerkennungstheoretischen Implikation einer solchen Szene vgl. Abschn. 3.3.3.2.
} 
of a low ridge, other eyes watched“ (19). Clayton und seine Frau „,kept a sharp lookout", doch durch den Wechsel zur tierlichen Perspektive im vierten Kapitel „The Apes“ (29 ff.) werden sie selbst zu den Beobachteten. Später ist es vor allem Tarzan, der die in das Gebiet der Affen einwandernden afrikanischen Menschen in ihrem Dorf ebenso beobachtet und studiert wie die neuen Weißen am Strand (William Cecil Clayton, Jane Porter und ihr Vater sowie dessen Assistent) und die nächste Schiffsladung an Meuterern, die an der Küste ihren Schatz vergraben.

Man könnte versucht sein, Tarzan derart als Beobachterfigur und sein Aufwachsen unter Affen sowie seine Selbstentwicklung als radikal teilnehmende Beobachtung zu lesen. Schließlich ist er in der privilegierten Position, in einer Umkehrung der entwicklungspsychologischen Aufzuchtexperimente ${ }^{26}$ mit Affen von klein an am äffischen Leben und Sein zu partizipieren und zu erlernen, was es heißt, Affe zu sein. Doch dieser Lesart steht zweierlei entgegen: Erstens nutzt Tarzan die Beobachtung instrumentell anders als die Ethologie. Die Beobachtung seines Umfelds sowie die Beobachtung neuer Phänomene bringen ihm neues Wissen, neue Kenntnisse und schließlich neue Fertigkeiten. Burroughs lässt allerdings deutlich werden, dass Tarzan seine Beobachtungsgabe nicht dazu nutzt, sein Gegenüber ethologisch zu erfassen. Tarzan nutzt sie rein dazu, sich selbst von anderen zu scheiden, diese zu manipulieren und $\mathrm{zu}$ seinem eigenen Nutzen zu handeln. Wenn man Tarzan überhaupt als einen teilnehmenden Beobachter beurteilen möchte, kennt er im Gegensatz zu den Feldforschenden nur die Empathie eines Psychopathen. ${ }^{27}$ Seine Beobachtungen als ,interested spectator“ (70) zeigen ihn zwar auch auf der Suche nach Einsicht in das Verhalten anderer, sie sind aber befreit von Mitgefühl. ${ }^{28}$ Damit entsprechen sie einem neueren Begriff der Empathie als menschlicher Fähigkeit, die, so Fritz Breithaupt, ,zunächst

\footnotetext{
${ }^{26}$ Siehe bspw. Yerkes, Robert M.: Almost Human. New York/London 1925; Kellogg, Winthrop N.: Humanizing the Ape. In: Psychological Review 38 (1931), S. 160-176; Kellogg, Winthrop N.: Chimpanzees in Experimental Homes. In: The Psychological Record 18 (1968), S. 489498.

${ }^{27}$ Während Psychopathie/Soziopathie klinisch zunächst mit einem Mangel an Empathie assoziiert wurde, suggerieren neuere Studien ein Missverhältnis unterschiedlicher affektiver und kognitiver Prozesse. Vgl. Hare, Robert D.: The Hare Psychopathy Checklist - Revised. Toronto 1991; Meffert, Harma u. a.: Reduced spontaneous but relatively normal deliberate vicarious representations in psychopathy. In: Brain 136 (2013), S. 2550-2562; Blair, R. J. R. u. a.: Emotion Attributions in the Psychopath. In: Personality and Individual Differences 19/4 (1995), S. 431-437; Blair, R. J. R. und D. G. Mitchell: Psychopathy, Attention and Emotion. In: Psychological Medicine 39/4 (2009), S. 543-555; Keysers, Christian: Inside the Mind of a Psychopath - Empathic, But Not Always. In: Psychology Today (2013), https://www.psychologytoday.com/intl/blog/the-empathic-brain/201307/inside-the-mind-psychopath-empathic-not-always (13.04.2018).

${ }^{28}$ Tarzan ist somit trotz der Ähnlichkeit der Formulierung auch eben nicht mit Adam Smiths ,impartial spectator“ zu assoziieren, der, so hat Ronge gezeigt, ,internalisierte[n] Repräsentation eines mit uns mitfühlenden Fremden“. Ronge, Bastian: Sympathetische Alterität. Die Figur des impartial spectator bei Adam Smith. In: Elisabeth Johanna Koehn u. a. (Hg.): Andersheit um 1800. Figuren - Theorien - Darstellungsformen. München 2011, S. 107-121, hier S. 112.
} 
und vor allem demjenigen [dient], der Empathie empfindet, und nicht dem, in den man sich einfühlt. "29 Miterleben und Mitfühlen sind für Breithaupt allererst ästhetische und epistemische Bereicherungen für den empathischen Menschen. Auch die jüngste, neurowissenschaftlich inspirierte Empathieforschung definiert Empathie nach Amy Coplan als ,a complex imaginative process in which an observer simulates another person's situated psychological states while maintaining clear self-other differentiation“. ${ }^{30}$ Im Evolutionsnarrativ des Romans dienen Tarzans Beobachtungen vorwiegend der Selbstexploration, der Selbsterkenntnis und der Selbstbereicherung. Gerade dies ist hier von Interesse, denn zweitens ist Tarzan der Protagonist dieses Romans und der Held ${ }^{31}$ seiner Erzählinstanz. Er ist dadurch zugleich das Objekt der narrativen Beobachtung. Das primatische Wesen, das hier von Geburt an beobachtet wird, ist: Tarzan. Tarzan of the Apes ist eine Tarzan- und damit auch eine primatologische Fallstudie.

Das Erkenntnisinteresse dieser Studie wird m. E. vor allem von seiner experimentellen Exposition geleitet. Wie bei realen Aufzuchtexperimenten lautet die Forschungsfrage, die Burroughs stellt: Was setzt sich durch - das genetische Erbe (,heredity“) oder die Aufzucht (,training“), ,nature“ oder ,nurture"? Diese Forschungsfrage besitzt bereits eine gewisse Ironie, da doch Tarzans „pedigree“, also seine Abstammung, durch privilegierte Bildung, Vermögen und Klasse entsteht und bestimmt ist. Seine ,nature' ist ohne die britische, viktorianische Klassengesellschaft nicht denkbar, somit durch ausgiebige ,nurture " entstanden. Umgekehrt ist Tarzans ,nurture' das bildungs- und kulturferne Aufwachsen im Tierreich unter Affen, durch welches er ganz auf sein ,natürliches ' Wesen zurückgeworfen ist. Tarzan ist als Figur bereits unvermeidlich ein Produkt jenes spätindustriellen Scheiterns der Scheidung von ,Natur' und ,Kultur' als gegenseitige Konstruktionsgestelle. ${ }^{32}$

\footnotetext{
${ }^{29}$ Breithaupt, Fritz: Die dunklen Seiten der Empathie. Berlin 2017, S. 14.

${ }^{30}$ Coplan, Amy: Understanding Empathy: Its Features and Effects. In: Dies./Peter Goldie (Hg.): Empathy. Philosophical and Psychological Perspectives. Oxford/New York 2011, S. 3-18, hier S. 5. Ein solcher Empathie-Begriff bietet sich an, um die besondere Befähigung einer psychopathischen Persönlichkeit zur ,Theory of Mind“ ohne affektive Übertragungsphänomene zu fassen.

${ }^{31}$ Tarzan ist nicht nur literarischer Titelheld sondern auch jener ältere Heros, der ,,von der Natur mit einer ansehnlichen Gestalt und ausnehmender Leibesstärcke begabet, durch tapffere Thaten Ruhm, erlanget, und sich über den gemeinen Stand derer Menschen erhoben“. Zedler, Johann Heinrich: Held, lat. Heros. In: Ders.: Grosses vollständiges Universal-Lexicon aller Wissenschaften und Künste. Bd. 12. Halle und Leipzig 1731-1754, S. 630-631.

${ }^{32}$ In Tarzan dämmert bereits - nur vom anderen Ende des politischen Spektrums her - Haraways Cyborg als Figur der Grenzauflösung, der „breached boundary“: „The cyborg appears in myth precisely where the boundary between human and animal is transgressed. [...] [C]yborgs signal disturbingly and pleasurably tight coupling“. Wie noch zu zeigen sein wird, ist Tarzan dieser Cyborg-Ontologie näher als Burroughs lieb sein kann: „The cyborg is resolutely committed to partiality, irony, intimacy, and perversity. It is opposition, utopian, and completely without innocence. [...] Nature and culture are reworked; the one can no longer be the resource for appropriation or incorporation by the other". Haraway, Donna: The Cyborg Manifesto. In: David Bell/Barbara M. Kennedy (Hg.): The Cybercultures Reader. London/New York 2001, S. 291-324, hier S. 292 f.
} 
Diese kategoriale Vermengung bildet sich in Tarzan ab. Durch das Aufzuchtexperiment ist Tarzans taxonomischer Status immer wieder fraglich. Zwar ist es auffällig, dass dieser Roman kein Enthüllungsroman sein will. Für die Leser/innen ist von Anfang an klar, dass Tarzan nicht nur ein Mensch, sondern auch dessen soziale Sonderform, ein Adliger, ist. Trotz dieses gleichsam expositionellen (Ab-) Sicherungsmechanismus stellt der Roman in der ,Wild Child'-Tradition ${ }^{33}$ ein interessantes entwicklungspsychologisches Problem auf: „Tarzan of the Apes had a man's figure and a man's brain, but he was an ape by training and environment"

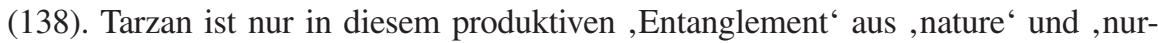
ture" denkbar.

Die Mischung von menschlicher Hard- und äffischer Software schlägt sich in Burroughs' Roman in einer Bezeichnungsuneindeutigkeit auch bei seinem Protagonisten nieder. Tarzan wird vom ,tiny man-child“ (28) zum ,ape-child“ (59) und ,,ape-man“ (87), ist zugleich ,the young lord“ (87) und ,only a beast of the jungle“ (180). In seiner Selbstwahrnehmung ist Tarzan ,their [der Affen] human king“. Für Jane ist er „the forest man“ (178) und „the wood man“ (179), dann ,a gentleman“ (180), für Clayton jedoch gleichzeitig ,a strange, half-savage creature of the jungle“ (179), wesentlich pejorativer noch ,our carrion-eating acquaintance“ und ,some half-demented castaway“ (180). Tarzan steht nicht nur zwischen den Arten, sondern gleich außerhalb der binären Tier-Mensch-Ordnung: So ist Tarzan den französischen Soldaten ,the black haired Giant“" (226) und für Jane in der Stunde der Not ein ,wild demi-god“ (180), „her wood-god“ (180) und ,the forest god“" (185). Doch der französische Captain ist es dann, der auf den Punkt bringt, weshalb Tarzan als Fallstudie des Menschlichen interessant wird: weil er, in den Worten des Captains, ,this super-man of yours“ (195) ist.

Dass Tarzans mensch-äffische Disposition ihn in Verbindung mit dem MissingLink-Diskurs und dem Verdacht der Diffundierung der Tier-Mensch-Grenze durch das biologisch Hybride stellt, macht der Text recht explizit. Sowohl die obigen Bezeichnungen verweisen darauf als auch handlungsintern die Tatsache, dass Tarzan selbst lange glaubt, Kala sei auch biologisch seine Mutter. Er bemüht sogar die Hilfe einer neuen kriminaltechnischen Errungenschaft - des Fingerabdrucks -, um der Wahrheit auf den Grund zu gehen und seine ,Reinartigkeit belegt zu bekommen. ${ }^{34}$ Tarzans einmalige physische und behaviorale Disposition

\footnotetext{
${ }^{33}$ Vgl. hierzu Douthwaite, Julia V: The Wild Girl, Natural Man, and the Monster: Dangerous Experiments in the Age of Enlightenment. Chicago 2002, S. $11 \mathrm{f}$.

${ }^{34}$ Dies wurde bereits mehrfach in der Forschung thematisiert. Es ist wieder D'Arnot, der Tarzan hier anhand des Fingerabdrucks versichert: „you are a pure man, and, I should say, the offspring of highly bred and intelligent parents“ (TA, S. 207). Siehe Griem, Julika: ,The answer to his life's riddle lay in these tiny marks'. Forensische Spuren in E. R. Burroughs', Tarzan of the Apes“. In: Gesine Krüger/Ruth Mayer/Marianne Sommer (Hg.): „Ich Tarzan. “ Affenmenschen und Menschenaffen zwischen Science und Fiction. Bielefeld 2008, S. 73-86; Abate, Michelle Ann: ,An Axe in the Hands of a Burly Negro Cleft the Captain from Forehead to Chin': Tarzan of the Apes and the American Urban Jungle. In: Annette Wannamaker/Michelle Ann Abate (Hg.): Global Perspectives on Tarzan: From King of the Jungle to International Icon. New York/ London 2012, S. 13-27.
} 
lässt ihn jedoch zu einem vor allen anderen ausgezeichneten Menschen werden: eine übersteigernde Veränderung der menschlichen Art, ein Übermensch. ${ }^{35}$ Durch den Text hindurch wird zu diesem Zweck mit einiger Redundanz Tarzans Überlegenheit hervorgehoben: „his superior being“ (39), „his superior intelligence and cunning“ (41), „,superior wisdom“ (88), ,,superior intellect“ (86), sein „healthy mind endowed by inheritance with more than ordinary reasoning powers" (52), gar „his divine power of reasoning“ (42). Dies lässt sich einerseits im Kontext der Sicherungsmechanismen lesen, die der Erzähler gegen die ,anthropological anxiety“ stemmt, welche aus der Nähe von Affe und Mensch erwächst. ${ }^{36}$ Die emphatische Erwähnung seiner Überlegenheit scheidet Tarzan offensichtlich von den Affen (und in deren Verlängerung von den Afrikaner/innen und den Angehörigen sozial niederer Stände). Doch sendet sie in ihrer Redundanz der Verwendung der Vokabeln ,superior' und ,supreme' auch andererseits ein Signal, weshalb uns dieser besondere Primat überhaupt $\mathrm{zu}$ interessieren hat. Der Roman Tarzan ist nicht nur eine Feier der anthropologischen Differenz; die Figur Tarzan ist auch ein von Burroughs entworfenes anthropologisches Ideal. Für dieses Ideal ist die äffische Prägung zugleich problematisch und unabkömmlich: Die Grundierung als ,primeval man“ garantiert zwar den „type of the strongly masculine“ (160) und ,the embodiment of physical perfection“ (111). Seine ,supremacy“ gründet sich aber nicht nur auf körperlicher Stärke, sondern auch auf einer Erbmasse an vorzüglichen physischen Charakteristika, ,the natural outcropping of many generations of fine breeding“"37 (eben jener, pedigree'), und,, an hereditary instinct of graciousness“ (166). Sowohl die überlegene menschliche Intelligenz als auch die Unverdorbenheit durch zivilisatorische Einflüsse, vor allem jedoch das mit den Affen verbundene Gewaltpotenzial lassen Tarzan schließlich zum „mighty fighter“, „mighty hunter“ (59), zum „,conqueror“ (87) und „human king“ (91) werden.

\footnotetext{
${ }^{35}$ Bislang wurde Tarzans idealisierter Ausnahmecharakter in der Forschung vorwiegend nur in Bezug auf seine Rolle in einem zeitgenössisch neuen Männlichkeitsideal und der damit verbundenen Zivilisationskritik untersucht. Siehe aber Haslam: Introduction, S. xxv; Abate, Michelle Ann: Bloody Murder: The Homicide Tradition in Children's Literature. Baltimore 2013, S. 99.

${ }^{36}$ Diese anthropologische Angst umfasst für Richter die beiden von Susan Bernstein vorgeschlagenen Angst-Formen: die ,anxiety of assimilation“ als „,distress of fusing of divisions“, „the fear of literally becoming the despised/desired Other", und die , anxiety of simianation" als „discomfort over evolutionary ties between humans and other primate species“, als ,, loss of difference in time, a return to a former evolutionary stage, cast as the - physical or mental assumption of ape-like qualities“. Richter: Literature after Darwin, S. 14, Hervorheb. im Original; siehe auch Bernstein, Susan: Ape Anxiety: Sensation Fiction, Evolution, and the Genre Question. In: Journal of Victorian Culture 6/2 (2001), S. 250-271, hier S. 255.

${ }^{37} \mathrm{Zu}$ den Eugenik-Bezügen von Tarzan vgl. Holtsmark, Erling: Tarzan and Tradition: Classical Myth in Popular Literature. Westport 1981, S. 147; Holtsmark, Erling: Edgar Rice Burroughs. Boston 1986, S. 49; Nies, Betsy L.: Eugenic Fantasies: Racial Ideology in the Literature and Popular Culture of the 1920's. New York 2002, S. 37 ff.
} 


\subsubsection{Im Affekt? Gewalt und moralische Ambivalenz}

Tarzans Charakterisierung als ,super-man ${ }^{638}$, als Übermensch, scheint hauptsächlich dafür verantwortlich, dass Edward Said von Tarzan als ,hodgepodge of

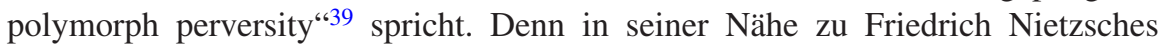
Übermensch als umwertendem Erneuerer der Menschheit fällt Tarzan als erstaunlich schwieriger Protagonist auf. ${ }^{40}$ Wie Ernst Benz in seinem Überblick zum Begriff des Übermenschen zusammenfasst, entsteht die post-christliche (Benz nennt es als Theologe die anti-christliche) Idee des Übermenschen nicht erst bei Friedrich Nietzsche. Schon bei Jean Paul findet sich die Idee einer revolutionären Hochmensch-Figur, die, so Benz, „mit ,Kraft' die Entwicklung der Verhältnisse $\mathrm{zu}$ beschleunigen und aus einer modrigen faulenden Welt eine grünende emporzutreiben weiß“.41 Hier wird gefordert, dem Übermenschen eine eigene Ethik zuzuerkennen. Eine weitere Gemeinsamkeit von Jean Paul und Nietzsche sieht Benz in der ,stille[n] Kälte des Herzens, mit dem ihn die Gewißheit seiner Sendung und der Glaube an seine Kraft erfüllt, die ihn instand setzt, von sich selbst und von anderen um seiner Idee und Sendung willen das höchste Opfer zu verlangen. ${ }^{642}$ Benz definiert daher in seiner Geschichte des Begriffs die Gestalt des Übermenschen nicht allein als ,,das schaffende Genie, das seine eigene Moral und seine eigenen sittlichen Geniezüge aufweist". ${ }^{43}$ Mit dem Eindringen des naturwissenschaftlichen Entwicklungsgedankens in die Anthropologie des 19. Jahrhunderts wird dieser idealistische Begriff angereichert, z. B. in der Form von Ralph Waldo Emersons ,plus-man“, der Tarzan präfiguriert als „,der Mann mit der überschüssigen Gesundheit, mit der strotzenden Vitalität, der Vollblütige [...]

\footnotetext{
${ }^{38}$ Jerry Siegels und Joe Shusters Superman (in: Action Comics 1. New York 1938) zieht zwar keine direkten Bezüge aus Nietzsches Übermensch-Begriff, ist aber wiederum nachweislich durch Edgar Rice Burroughs' Ideen beeinflusst. Vgl. Andrae, Thomas: Of Supermen and Kids with Dreams: A Rare Interview with the Creators of Superman: Jerry Siegel and Joe Schuster. In: Nemo, The Classic Comics Library 2, S. 6-19. Supermans Gesicht ist sogar dem seit 1932 als Tarzan besetzten Johnny Weissmüller nachempfunden. Vgl. Ricca, Brad: Super Boys: The Amazing Adventures of Jerry Siegel and Joe Shuster - The Creators of Superman. New York 2014.

${ }^{39}$ Said: Jungle Calling, S. 329.

${ }^{40}$ Burroughs bezieht sich intratextuell nicht explizit auf den späten Nietzsche. Es lässt sich jedoch auch nicht einfach mit Taliaferro behaupten, Burroughs „had never heard of Rousseau's noble savage or Nietzsche's Übermensch". Taliaferro: Tarzan Forever, S. 14, Hervorheb. im Original. Denn Nietzsches Gedanken werden spätestens seit der Jahrhundertwende auch für eine angelsächsische Öffentlichkeit diskursiv. Vgl. Abate: Bloody Murder, S. 99 f.

${ }^{41}$ Benz, Ernst: Das Bild des Übermenschen in der europäischen Geistesgeschichte. In: Ders. (Hg.): Der Übermensch. Eine Diskussion. Zürich/Stuttgart 1961, S. 19-162, hier S. 81.

${ }^{42}$ Benz: Das Bild des Übermenschen, S. $82 \mathrm{f}$.

${ }^{43}$ Benz: Das Bild des Übermenschen, S. 83.
} 
[,] der Erfolgreiche“. ${ }^{44}$ Nietzsches Übermensch ist zunächst in der vor-zarathustrischen Verwendung ein Ausnahme-Heros unter Verdacht des Geniekults. Beim späteren Nietzsche, vor allem im Zarathustra, greift der Übermensch „höher in die Sphäre des Übermenschlichen und tiefer in die Schicht des Untermenschlich-Dämonischen“ 45 hinein, und wird unter dem Eindruck der Evolutionstheorie das Produkt der freien Schöpfung und bewussten Züchtung unter Ausmerzung vor allem jener Eigenschaften, die mit der christlichen Sittenlehre zusammenhängen. Dies bedeutet für Nietzsche auch: „[D]as Böse ist des Menschen beste Kraft. ,Der Mensch muss besser und böser werden' [...] [.] Das Böseste ist nöthig zu des Übermenschen Bestem." ${ }^{6} 6$ Schließlich tritt der Übermensch unter der Frage „Wie wird der Mensch überwunden?“47 als, wie Benz zusammenfasst, „das innere Modell der gesamten Entwicklung des Lebens“ hervor, in der Vorstellung, „daß der zukünftige Schritt des Menschen zum Übermenschen bereits von Anfang an als Ziel der Gesamtentwicklung vorschwebte, die vom Tier zum Menschen, zum Übertier führte. “48

Ein solches inneres Modell der gesamten Entwicklung des Lebens zeigt uns Burroughs anhand von Tarzans Entwicklung vom Übertier (dem vor allen anderen Affen ausgezeichneten ,Überaffen') zum Übermenschen (der allen anderen Menschen überlegene Ausnahmeheros). Allerdings scheint die Transzendenz einer christlich-abendländischen Moral bei Burroughs weniger intendierte Botschaft als irritierender Begleiteffekt seiner Konzeption eines neuen Männlichkeits- als Menschheitsideals zu sein. Burroughs Erzähler versucht uns immer wieder der ethischen Vorzüglichkeit und der moralischen Überlegenheit dieses Helden zu versichern, bietet uns dabei aber Beobachtungsmomente, die dem vehement widersprechen. Dies macht sich vor allem bemerkbar an Tarzans Vorliebe für Gewalt und Verbrechen. Gesine Krüger geht so weit, Burroughs als „geradezu besessen von den Themen Grausamkeit, Tod und Gewalt “49 zu sehen und konstatiert eine lustvolle Darstellungsweise von Gewaltszenen. Michelle Anne Abate hat bereits herausgearbeitet, wie instrumental Gewalt für die Entwicklungsgeschichte Tarzans

\footnotetext{
${ }^{44}$ Benz: Das Bild des Übermenschen, S. 108. Dass Burroughs durch die amerikanischen Transzendentalisten Emerson und Thoreau inspiriert wurde, erwähnt Haslam: Introduction, S. xxf. Emersons ,plus man' unterscheidet sich jedoch von Nietzsches Übermenschen gerade darin, dass er innerhalb einer eher demokratisch zu nennenden Vorstellung seinen Mitmenschen dient und einer höheren Ethik folgt. Vgl. Soressi, Beniamino: Europe in Emerson and Emerson in Europe. In: Jean Mclure Mudge (Hg.): Mr. Emerson's Revolution. Cambridge 2015, S. 325-372, hier S. $345 \mathrm{ff}$.

${ }^{45}$ Benz: Das Bild des Übermenschen, S. 118.

${ }^{46}$ Nietzsche: Zarathustra, S. 359.

${ }^{47}$ Nietzsche: Zarathustra, S. 357.

${ }^{48}$ Benz: Das Bild des Übermenschen, S. 131.

${ }^{49}$ Krüger: Schrift und Gewalt, S. 31.
} 
ist: „[F]ar from a merely sensational element in the narrative" 50 spielen die Gewalt-Episoden eine entscheidende Rolle für die Formierung des Titelhelden, die Entwicklung des Romanplots und die Konstruktion seiner Themen: „Tarzan of the Apes is presented as a superior being, and he is never more physically powerful or socially impressive than when he is engaged in the act of murder. [...] [H] is status as a great man is predicated on his ability as a fierce killer" ${ }^{51}$ Abates These lautet, dass Tarzan sich nicht allein aufgrund seiner Abstammung von den ,höheren“ Rassen als ,superior being ' erweist, sondern auch, weil er keines der atavistischen Elemente zeigt, die der Kriminalanthropologe Cesare Lombroso zeitgenössisch mit chronischen Dieben und Gewohnheitsmördern assoziierte. Für Abate ist Tarzan daher als ,the anti-Lombrosian man“52 zu untersuchen.

Doch was bedeutet es, dass Tarzan als Anti-Lombrosianer so handelt, wie er handelt; dass sein Verhalten sich nicht in einem , untermenschlichen“ Äußeren manifestiert? ${ }^{53}$ Es lässt sich weiterdenken: Gerade durch seine nicht an zeitgenössische Erklärungsmuster gebundenen Gewalthandlungen stemmt sich Tarzan als besonderer Primat gegen das innovative Wissen der sozialdarwinistischen Kriminalanthropologie. Er transzendiert nicht nur das Wissen über Kriminalität, sondern auch die damit verbundenen Moralvorstellungen. Der Übermensch gerät Burroughs so gefährlich in die Nähe der Psychopathie; er ist nicht Robert Musils Moosbrugger sondern Bret Easton Ellis' Patrick Bateman. ${ }^{54}$

Es ist gerade Burroughs' übermäßige Betonung nicht nur von Tarzans Herkunft (,pedigree'), sondern vor allem seines genetischen Erbes (,hereditary'), die den Verdacht einer solchen Persönlichkeitsstörung nahelegt. ${ }^{55}$ Tarzan setzt seine ,superior intelligence and cunning“ (41) überwiegend ein, um anderen zu schaden. Gewalt ist bereits früh in Tarzans Biografie angelegt. So erlaubt ihm seine höhere Intelligenz, in den Worten des Erzählers, „to invent a thousand diabolical tricks“ (41). Sobald Tarzan bspw. die Einsicht und Fähigkeit erlangt hat, Seile herzustellen, daraus

\footnotetext{
${ }^{50}$ Abate: Bloody Murder, S. 94.

${ }^{51}$ Abate: Bloody Murder.

${ }^{52}$ Abate: Bloody Murder, S. 95.

${ }^{53}$ In der englischen Übersetzung von Lombrosos L'uomo deliquente heißt es u. a. ,the criminal an atavistic being who reproduces in his person the ferocious instincts of primitive humanity and the inferior animals“ sowie: „Thus was explained the origin of the enormous jaws, strong canines, prominent zygomæ, and strongly developed orbital arches [...], for these peculiarities are common to carnivores and savages [...]. The other anomalies exhibited by criminals - the scanty beard as opposed to the general hairiness of the body, prehensile foot, diminished number of lines in the palm of the hand, cheek-pouches, [...] flattened nose and angular or sugar-loaf form of the skull, common to criminals and apes“. Lombroso, Cesare: Criminal Man, According to the Classification of Cesare Lombroso. New York/London 1911 (ital. 1876), S. xv, 7.

${ }^{54}$ Siehe Musil, Robert: Der Mann ohne Eigenschaften. Bd. 1. Hg. von Adolf Frisé. Reinbek bei Hamburg 1978 (Gesammelte Werke); Ellis, Bret Easton: American Psycho. New York 1991.

${ }^{55}$ Neuere Studien aus dem Bereich der Psychopathie-Forschung verweisen immer stärker auf genetische Faktoren der Persönlichkeitsstörung. Vgl. Hare, Robert D. und Craig S. Neumann: Psychopathy as a Clinical and Empirical Construct. In: Annual review of Clinical Psychology 4 (2008), S. $217-$ 246, hier S. 238.
} 
Schlingen zu formen und sie als Strick zu nutzen, ist ihm dies nicht nur ein ,new game, a fine game“" (42), sondern er wendet diesen neuen ,Trick' sogleich darauf an, seinen ungeliebten äffischen Stiefvater zu foltern: „In sleep, upon the march, night or day, he never knew when that quiet noose would slip about his neck and nearly choke the life out of him“ (42). Für die endgültige Selbst-Findung Tarzans ist schließlich ein besonderer Tötungsakt notwendig: Tarzan wird zu seinem titelgebenden Selbst - „Tarzan of the Apes“ (58) - erst durch die Tötung eben jenes Stiefvaters. Tarzan erkennt sich selbst mit den Worten: „I am Tarzan. [...]. I am a great killer“ (58). Hier wird in Tarzan durch den ersten Mord - den (Stief-)Vatermord - eine essenzielle Blutlust geweckt, die sich fortan durch die Handlung(en) des Romans ziehen wird, als handle es sich um die Erzählung von einem Serienmörder. ${ }^{56}$ Die nächste Tötung wird noch auf der gleichen Seite geplant: „But into the mind of Tarzan a great plan sprang. He had killed the fierce Tublat, so was he not therefore a mighty fighter? Now he would track down the crafty Sabor and slay her likewise. He would be a mighty hunter, also“" (58).

Es gibt ein affektives Fundament für Tarzans Gewalthandlungen, sie könnten also rechtfertigend als Affekttaten gelesen werden. Doch Burroughs macht uns dies nicht ganz einfach. Als Kulonga, einer der Afrikaner, Tarzans äffische Mutter Kala tötet, ist ,Tarzan's grief and anger [...] unbounded. He roared out his hideous challenge time and again. He beat upon his great chest with his clenched fists, and then he fell upon the body of Kala and sobbed out the pitiful sorrowing of his lonely heart" (68). Diese schmerzliche Trauer und das damit verbundene Rachegelüst angesichts des „dead body of his slain mother“ (68) erscheinen nur allzu verständlich. Doch dann wird uns berichtet:

After the first outbursts of grief Tarzan controlled himself [...]. His desire to kill burned fiercely in his wild breast, but his desire to learn was even greater. He would follow this savage creature for a while and know from whence he came. He could kill him at his leisure later [...]. (70, Hervorheb. MS)

Diese Melange aus Mordlust und Mäßigung ist ausschlaggebend: Der vorausschauende Aufschub der affektiven Befriedigung des Rachebedürfnisses macht aus heutiger kriminalrechtlicher Perspektive betrachtet - die Tötung zum Mord. ${ }^{57}$

\footnotetext{
${ }^{56}$ Tatsächlich ist Tarzans Dezimierung von Mbongas Dorf als das Wirken eines Serienmörders oder ,one-man lynchmob“ lesbar, wie Abate vorführt. Vgl. Abate: Bloody Murder, S. 97 ff. Der betreffende Kapitel-Titel scheint dessen Pseudonym anzubieten: „The Fear-Phantom“ (TA, S. 74 ff.).

${ }^{57}$ Das Schweizer Strafrecht unterscheidet den Totschlag vom Mord durch die Handlung ,in einer nach den Umständen entschuldbaren heftigen Gemütsbewegung“. Schweizerisches Strafgesetzbuch, Art. 112 Tötung/Todschlag, siehe https://www.admin.ch/opc/de/classified-compilation/19370083/index.html\#a112, (12.05.2018). Das deutsche Strafgesetzbuch beinhaltet noch immer den Heimtücke-Hinweis in seiner Mord-Definition. Strafgesetzbuch, § 211 Mord, siehe https://www.gesetze-im-internet.de/stgb/_211.html, (12.05.2018). Das US-Strafrecht definierte Mord einst durch „malice aforethought“, mittlerweile als „,criminal homicide [that] is committed purposely, knowingly, or recklessly under circumstances manifesting extreme indifference to the value of human life"; ,manslaughter" ist dagegen „,comitted under the influence of extreme mental or emotional disturbance“, siehe Section 210.3., Model Penal Code. Offical Draft and Explanatory Notes. Philadelphia 1985, S. 117 ff.
} 
Dass Tarzan sich dann auch noch der Kleidung und Waffen des getöteten Kulongas bemächtigt (72), verstärkt den Verdacht nur.

Wie sich im Verlauf der Handlung zeigt, werden Habgier und Rassismus zunehmend zu Hauptmotiven für Tarzans ,killing spree‘. Gerade das Töten der afrikanischen ,Eingeborenen“ geht Tarzan besonders leicht von der Hand, und immer geht es um die Eroberung von Waffen oder Nahrung; in diesem Zusammenhang heißt es: „Tarzan of the Apes was no sentimentalist. He knew nothing of the brotherhood of man“ (74) - ein deutlicher Verweis auf den abolitionistischen Slogan „Am I not a man and a brother?" ${ }^{58}$ Burroughs scheint jedoch selbst einen Erklärungsbedarf für Tarzans Morden zu sehen und versucht dieses erzählerisch in mehreren Passagen einzuhegen. In diesen steht Tarzans Emotionalität in Zusammenhang mit seiner Gewaltausübung und seiner Physis im Mittelpunkt. Zu Beginn des Kapitels, das seine wiederholten Angriffe auf Mbongas Dorf schildert, heißt es über Tarzan, er empfinde ,[a]ll things outside his tribe“ als „deadly enemies“ (74), und weiter:

\begin{abstract}
And he realized all this without malice or hatred. To kill was the law of the wild world he knew. Few were his primitive pleasures, but the greatest of these was to hunt and kill, and so he accorded to others the right to cherish the same desire as he, even though he himself might be the object of their hunt.

His strange life had left him neither morose nor bloodthirsty. That he joyed in killing, and that he killed with a joyous laugh upon his handsome lips betokened no innate cruelty. He killed for food most often, but, being a man, he sometimes killed for pleasure, a thing which no other animal does; for it has remained for man alone among all creatures to kill senselessly and wantonly for the mere pleasure of inflicting suffering and death.

And when he killed for revenge, or in self-defense, he did that also without hysteria, but it was a very businesslike proceeding which admitted no levity. (74, Hervorheb. MS)
\end{abstract}

Die innere Widersprüchlichkeit dieser Textstelle ist bemerkenswert. Zunächst ist das Töten normativ: „To kill was the law of the wild world he knew“, dann jedoch ein Vergnügen, die größte der ,primitive pleasures“ ist es „to hunt and kill“". Dass er also ,,joyed in killing“, ist Tarzan aber nicht als ,innate cruelty“ auszulegen. Gleichzeitig tötet Tarzan manchmal „for pleasure“; das Vergnügen ist nun aber prompt als Differenzkriterium nicht mehr tierlich-natürlich, sondern menschlich-kulturell. Eigentlich ist sogar, so impliziert die Textstelle, „to kill senselessly and wantonly for the mere pleasure of inflicting suffering and death" zu kritisieren. Zudem wird positiv hervorgehoben, dass Tarzan nicht etwa im Status unberechenbarer und unbeherrschbarer Emotionalität (,hysteria“) tötet, sondern selbst wenn aus Rache oder Selbstverteidigung (als gegensätzliche Konzepte der Rechtfertigung) immer geschäftsmäßig (,,businesslike“), also routiniert aber, das überrascht angesichts seiner Lustfähigkeit, ohne Ungezwungenheit (,levity“).

\footnotetext{
${ }^{58}$ Zudem setzt Burroughs auch mit der von Tarzan für schwarze Menschen und Tiere favorisierten Tötungsweise, dem Lynching, einen deutlichen Hinweis. Vgl. Bederman: Manliness and Civilization, S. 224 f.; Abate: Bloody Murder, S. 83 f.
} 
Die zweite hier als Beispiel dienende Passage ist logisch ähnlich herausfordernd. Als Jane Porter zunächst von Tarzans Adoptivbruder Terkoz entführt, dann von Tarzan befreit, aber weiterhin verschleppt wird, wächst Janes Begehren für Tarzan mit der Nähe des Blicks. Burroughs sieht hier die Notwendigkeit, die Diskrepanz zwischen Tarzans moralisch ambivalentem Verhalten und seinem lombrosianisch unverdächtigen Äußeren zu erläutern:

\begin{abstract}
A perfect type of the strongly masculine, unmarred by dissipation, or brutal or degrading passions. For, though Tarzan of the Apes was a killer of man and of beasts, he killed as the hunter kills, dispassionately, except on those rare occasions when he had killed for hate - though not the brooding, malevolent hate which marks the features of its own with hideous lines.

When Tarzan killed he more often smiled than scowled, and smiles are the foundation of beauty. (160, Hervorheb. MS)
\end{abstract}

Wieder scheinen hier Moral und Affektivität in einem merkwürdigen Widerstreit zu stehen: Tarzan ist „unmarred“ zugleich von den Zerstreuungen der Zivilisation (,disspiation“) und den ungeschliffenen „passions“. Er wird als „,killer of man and of beasts" anerkannt, aber für diese Argumentation ist es wichtig - im Widerspruch zum vorherigen Zitat - hervorzuheben, er töte ,as the hunter“ leidenschaftslos (,dispassionately“). Sogleich wird jedoch eine Ausnahme (,except“) hergestellt, die hochaffektiv scheint: „When he had killed for hate“. Doch, wie uns versichert wird, es handelt sich um die ,gute“ Art von Hass und „,not the brooding, malevolent hate". Denn diese, und damit soll der Bogen wieder geschlossen werden, entstelle das Gesicht - und Tarzan ist nun einmal vollständig schön. Das non sequitur des letzten Satzes - ,he more often smiled than scowled, and smiles are the foundation of beauty“, zeigt uns das Bild eines lächelnden Mörders. Dies ist an dieser Stelle ein umso irritierenderes Bild, als das Motiv einer Vergewaltigung über der Handlung schwebt. Nicht nur Terkoz entführt Jane, um sie zu einer seiner Frauen zu machen. Während Jane selig in Tarzans Armen durch die Baumwipfel fliegt und in der ,vision of that handsome, winning face“ (160) aufgeht, denkt Tarzan darüber nach, ob er sie nun seinerseits vergewaltigen soll:

\footnotetext{
As Tarzan moved steadily onward his mind was occupied with many strange and new thoughts. [...] He knew why the ape had not killed her, and he commenced to compare his intentions with those of Terkoz.

True, it was the order of the jungle for the male to take his mate by force; but could Tarzan be guided by the laws of the beasts? Was not Tarzan a Man? But how did men do? He was puzzled; for he did not know. (161, Hervorheb. MS)
}

Die Paarung „by force“ wird von Burroughs doppelt legitimiert, als „order of the jungle" und „laws of the beasts“, denn als Option wird sie von Tarzan durchaus in Betracht gezogen (,his intentions“). Zugleich wird die beidseitig freiwillige Paarung hier als mögliches anthropologisches Differenzkriterium aufgebaut. Wieder betont Burroughs, dass letztlich ,heredity spoke louder than training“ (166), denn: „He had not in one swift transition become a polished gentleman from a savage ape-man, but at last the instincts of the former predominated" (166). 
Tarzan bedeutet Jane wortlos, sie habe von ihm nichts zu befürchten. Diese Instinkte des Gentlemans, das ist hier nicht unwichtig, sind jedoch im Text nicht deutlich moralische. Tarzan handelt nicht etwa aufgrund eines angeborenen menschlichen Moralempfindens. Tarzan ist noch ratlos (,,puzzled“) darüber, „how did men do". Aber er liest aus Janes Körpersprache, dass sie wohl hier nicht unbedingt vergewaltigt werden will. Dies dürfte eine der wenigen Passagen im Roman sein, in denen Empathie als ,das Aufschlüsseln der Emotionen, Affekte und Aktionen des anderen als anderen"59 zu positivem Ausgang eingesetzt wird. Überwiegend nutzt Tarzan seine Form von Empathie schlicht als Technik, die, mit Coplans Definition, zu Folgendem dient: ,[It] provides an observer with knowledge of another person's thoughts, feelings, and behaviour - knowledge that may (though need not) subsequently figure into the explanations, predictions, and even the actions of the observer". ${ }^{60}$ Als Teil von ,superior intelligence and cunning“ (41) ist dies in Tarzan eine Fähigkeit, deren hedonistische Anteile größer als ihre affektiven sind. Tarzan schafft es ausnehmend gut, Empathie von Mitgefühl zu trennen. ${ }^{61}$

Der literarischen Figur soll hier nicht unnötig eine Persönlichkeitsstörung diagnostiziert werden. Doch Tarzan steht im besten Fall jenseits einer zeitgenössischen menschlichen Ethik und diesseits einer ,jungle ethics“ (72). In Tarzan werden wir mit einer Ethik konfrontiert, die zwischen einem (ambigen) angeborenen Ehrverständnis und einem zivilisatorischen Rechtskorsett unterscheidet. Konsequenterweise wird Tarzans Töten erst ,in der Zivilisation“ in Amerika handlungsintern als Verbrechen thematisiert. Als er Janes Zwangsverlobten Cranler angreift, weiß Jane, „that murder lay in that savage heart“, und „,realized the stern retribution which justice metes to the murderer" (235). Sie unterbindet das Geschehen mit den Worten „I do not wish you to become a murderer“ (235), als sei dies der erste Mord, den Tarzan begehen würde. Trotz diverser Verweise auf die ,rights ', ,laws ‘ , order' oder ,jungle ethics“ wird dadurch nachträglich enthüllt, dass alle anderen Tötungen in einem rechtsfreien Raum - dem Dschungel - begangen

\footnotetext{
${ }^{59}$ Breithaupt: Kulturen der Empathie, S. 31.

${ }^{60}$ Coplan: Understanding Empathy, S. 17.

${ }^{61}$ Besonders deutlich wird dies wieder anhand seiner Begegnungen mit Mbongas Dorf. Die kannibalistischen Zeremonien der Einwohner (TA, S. 81 ff.) beobachtet und beurteilt Tarzan („He saw that these people were more wicked than his own apes, and as savage and cruel as Sabor, herself“,S. 81). Er betrachtet sie jedoch „,with complacency“ (S. 174) und sieht keinerlei Veranlassung den Opfern zu helfen. Im Gegenteil, „only occasionally interfering for the pleasure of baiting the blacks" (S. 174), nutzt er hier empathische Einsicht in das Verhalten des Stammes, um aus Lust, Habgier oder rassistischem Vorurteil zu töten und sich zu bereichern. Erst als es der Franzose D'Arnot ist, der Opfer des Kannibalismus zu werden droht, wird in Tarzan Anteilnahme geweckt. Und Burroughs macht den Grund dafür explizit: „Heretofore their victims had been man of their own color. Tonight it was different - white men, men of Tarzan's own race - might be even now suffering the agonies of torture" (S. 174). Selbst wenn Tarzan seinen weißen Artgenossen zur Hilfe eilt - Clayton, dem Professor, Jane, den Franzosen - gleitet die Erzählperspektive tendenziell zur externen Fokalisierung und verschließt sich einer Erkundung von Tarzans Affektivität jenseits von Hass (auf seine ,Feinde') und Begehren (für Jane und für Waffen).
} 
worden sind. Der Ethik folgend, die Burrough hier propagiert, wird der Mord erst im Raum des Rechts zu einem solchen. Die ,jungle ethics` dagegen gewährt der Mordlust ihren Lauf, sofern ihre Opfer Tiere, schwarze Menschen oder ,Asoziale“ sind. Die ideale Verkörperung einer solchen Ethik ist in diesem Roman eine Figur, die sich besser - aber nicht weniger irritierend - erschließt, wenn man sie als vom ,Recht der Natur' legitimierten, kaum gezügelten Psychopathen betrachtet. ${ }^{62}$

\subsubsection{Hodgepodge: Irritierende Vielfalt der Formen im Tarzan}

Sianne Ngai beschreibt Irritation als ,a strangely cutaneous feeling located at the border between emotional and bodily experience, and thus ,superficial ' in more ways than one". ${ }^{63}$ Das Gefühl der Irritation erscheint in dieser Konzeption ähnlich wie in Burroughs’ Bild von ,brooding, malevolent hate which marks the features of its own with hideous lines“ (160) als die zeichenhafte Verbindung zweier Systeme - des emotional-affektiven und des körperlich-physischen. Mit Ngai lässt sich diese Konzeption auch auf den Text anwenden: Irritation kann dann ,gelesen“ werden als zugleich ein Merkmal des Textes selbst, der keine geschlossene Form mehr anbietet, sondern durch Formvielfalt oder -konkurrenz auffällig, uneben, scheckig etc. wird, und als Wirkung bzw. Reaktion die durch solche Dissonanz hervorgerufen wird, als ein affektives Signal, das schlicht darauf verweist, hier ,stimme etwas nicht'. Versteht man ,Irritation“im/am Text als Anzeichen einer Art Bruch in der Affektlogik, so lässt sich eine derartige Störung sowohl ,subkutan“ auf der Ebene affektiver Gestimmtheit als auch gleichsam ,kutan“ auf der Textoberfläche untersuchen. Die, subkutane“ Irritation, die Edgar Rice Burroughs' Tarzan durch den Widerstreit der vorzüglichen Behandlung des Protagonisten durch den Erzähler mit der moralischen Ambivalenz, die das Handeln dieser Figur bestimmt, und die darin inhärente Aussage des Textes (als Entwurf eines neuen Männlichkeits-/Menschenideals jenseits einer zivilisatorischen Ethik) hervorruft, trifft auf ein ,kutanes' Phänomen, das im Sinne von Metelmanns und Lorens Ästhetik der Irritation einen entfremdenden Effekt hat: Auf der Textoberfläche streiten verschiedene Genres miteinander, reiben sich und erzeugen als Versatzstücke Irritationen.

Denn was ist dieser Roman auf der Ebene des Genres? Im Rahmen der vorliegenden Arbeit interessierte eine inhaltlich fokussierte Lesart als primatologische und ethologische (Fall-)Studie. Aber Tarzan hat wesentlich mehr Genre-Merkmale und ist darin mindestens so polymorph wie sein Protagonist. Er ist deutlich als

\footnotetext{
${ }^{62}$ Die Hare Psychopathy Checklist überprüft 20 Persönlichkeitszüge, von denen Tarzan etliche aufweist: Von einem erheblich übersteigerten Selbstwertgefühl und einem Stimulationsbedürfnis über betrügerisches und manipulatives Verhalten, den Mangel an Schuldbewusstsein, Gefühlskälte und einen parasitischen Lebensstil, frühe Verhaltensauffälligkeiten und wenig Verhaltenskontrolle, bis hin zu Impulsivität, Verantwortungslosigkeit und krimineller Vielseitigkeit.

${ }^{63}$ Ngai: Ugly Feelings, S. 185.
} 
Abenteuerroman angelegt, dabei zu Teilen der Robinsonade und (mit dem Motiv der wiederholten Meuterei und der Schatzsuche) den „printed stories of the sea“ (7) verschrieben, die er selbst erwähnt. Er ist zugleich eine Erzählung von der Evolution des Menschen (eine Primatographie) und ein Bildungsroman. ${ }^{64}$ Durch sein Setting und Teile des Plots ist er aber auch ein Kolonialroman: „From the records of the Colonial Office [...] we learn that a certain young English nobleman, whom we shall call John Clayton, Lord Greystoke, was commissioned to make a peculiarly delicate investigation of conditions in a British West Coast African Colony“ (5). Durch die Figurenkonstellation Jane-Tarzan handelt es sich um einen Liebesroman - und durch die Ergänzung um William Cecil Clayton hat dieser das Potenzial zu einem Eifersuchtsdrama. Der ,marriage plot ' gegen Ende des Romans (230 ff.) scheint zudem aus viktorianischen Gesellschaftsromanen entlehnt. Die Einführung kriminaltechnischer Methoden (und Tarzans ,killing spree“) verleiht ihm Züge des Kriminalromans oder der Mystery Story. In seinem Fokus auch auf die Perspektive der Affen nimmt Tarzan die Tiergeschichte auf. Es gibt zudem Elemente der Jagdliteratur (das Kapitel „The Tree Top Hunter“, 58 ff.), der Kriegs-Erzählung (in der Schlacht zwischen den französischen Soldaten und den afrikanischen ,Eingeborenen'), des ödipalen Dramas, der ,Buddy'-Motivik des Westerns (die Freundschaft zwischen D'Arnot und Tarzan und ihre Ankunft im Dorf) und der rassistischen Farce (die Figur Esmeralda scheint allein zu diesem Zweck zu existieren). Zudem werden uns Tagebuchaufzeichnungen (28; 207 f.) und Briefe (141 ff.; 187) als Mittel der Erzählung untergeschoben und nicht einmal der ,Ton` dieser Erzählung ist einheitlich: So schwankt Burroughs' Erzählinstanz zwischen der vermeintlichen Sprache der, high culture ${ }^{65}$ und den expliziten Details der, pulp fiction'. ${ }^{6}$

Allein schon die Erzählstimme in Tarzan ist irritierend: Im ersten Satz des Romans wird durch ein „I“ ein Ich-Erzähler eingeführt, der eine Rahmenhandlung andeutet. Dieser homodiegetische Ich-Erzähler verschwindet dann jedoch im weiteren Romantext zugunsten des heterodiegetischen, unfokalisierten Erzählens, dessen Instanz eben diese Rahmenhandlung wieder zu vergessen scheint. Der Roman-Einstieg wird dabei genutzt, um Uneindeutigkeit über die Verlässlichkeit

\footnotetext{
${ }^{64}$ Tarzans (Selbst-)Bildungsgeschichte, in der er sich eigenständig das Lesen und Schreiben beibringt, sowie aus der Lesefibel Rassismus lernt, wurde bereits in den Fokus gerückt, vgl. Romören, Rolf: The Light of Knowledge - In the Midst of the Jungle: How Tarzan Became a Man. In: CREArTA 2/2 (2001), S. 60-67.

${ }^{65}$ So verwendet Burroughs bspw. altertümelnde Formen und Wendungen wie „builded“ (TA, S. 114) statt , built".

${ }^{66}$ Besonders deutlich wird dies in der ,Pathosgeste' der Frau, um die sich zwei Männer - Terkoz und Tarzan - bis auf den Tod bekämpfen: „Jane Porter - her lithe, young form flattened against the trunk of a great tree, her hands tight pressed against her rising and falling bosom, and her eyes wide with mingled horror, fascination, fear, and admiration - watched the primordial ape battle with the primeval man for possession of a woman - for her“ (TA, S. 153); „He did what no red-blooded man needs lessons in doing. He took his woman in his arms and smothered her upturned, panting lips with kisses" (S. 154).
} 
der Erzählung herzustellen. Das Ich ,had this story from one who had no business to tell it to me, or to any other“ (5). Der „narrator“ ist hier jemand anderes, der weinselig berichtete, während der Ich-Erzähler sich selbst durch seine „skeptical incredulity“ und ,doubtfulness“ nicht nur auszeichnet, sondern ,,written evidence“ erlangt. Dem „strange tale“ soll ,,in the form of musty manuscripts, and dry official records of the British Colonial Office“ und ,[t]he yellow, mildewed pages of the diary of a man long dead“ (5) Authentizität verliehen werden. Doch der Erzähler lässt hier nach wie vor Raum dafür, dass es sich schlicht um eine schillernde Fiktion handelt:

I do not say the story is true, for I did not witness the happenings which it portrays, but the fact that in the telling of it to you I have taken fictitious names for the principal characters quite sufficiently evidences the sincerity of my own belief that it may be true. [...] If you do not find it credible you will at least be as one with me in acknowledging that it is unique, remarkable, and interesting. (5, Hervorheb. im Original)

Selbst hier scheint Burroughs' verquere Logik auf: Denn weshalb sollte ausgerechnet die Tatsache der „fictitious names“ ein Indiz für Faktualität statt Fiktionalität sein?

\subsubsection{Affektpoetik der irritierenden Form}

Saids Kritik lässt sich also sowohl in Bezug auf Tarzans Protagonisten wie auf die formale Oberfläche des Textes aufgreifen. Was für den Roman als „hodgepodge of polymorph perversity" problematisch wird, ist, so sollen die folgenden Kapitel zeigen, auch bei den primatologischen Forschungsmemoiren signifikant. Dabei stehen die Textform bzw. das Genre als Arten des Erzählens von wissenschaftlicher Forschung und emotionaler Herausforderung im Mittelpunkt. Die Vielfalt an Text(sub-)formen soll als Verweis auf eine affektive Irritation gelesen werden. Es wird jedoch nicht ihr Potenzial als Entfremdungsinstrumente untersucht, wie Metelmann und Loren dies ansetzen und wie Burroughs Tarzan of the Apes es verdeutlicht. Im Gegenteil soll im Folgenden mit der neueren Genre-Forschung ,Genre“ nicht länger nur als ,form and text type“67 entlang einer fryeschen ,Theory of Modes' gelesen werden, die trotz aller Offenheit für Mischformen doch deutlich ideologische $\mathrm{Zu}$ - und Einordnungen vornimmt. ${ }^{68}$ Mit Amy Devitt

\footnotetext{
${ }^{67}$ Devitt: Generalizing about Genre, S. 573.

${ }^{68}$ Trotz dieser grundsätzlichen Vorbehalte soll das Potenzial von Fryes Genre-Theorie weiterhin genutzt werden, denn sie bietet mit ihrem Verständnis von Genre als ,intention of producing a specific kind of verbal structure“, „determined by the conditions established between the poet and his public“, die Grundlage für die produktionsästhetische Weiterentwicklung von Genre-Konzepten. Frye: Anatomy of Criticism, S. 347 f.
} 
möchte ich Genre/Textformen als „dynamic patterning of human experience, as one of the concepts that enable us to construct our writing world",69 und mit Burkhard Meyer-Sickendiek als „Medien, in denen sich menschliche Affekte artikuliert, transformiert oder kanalisiert haben“, ${ }^{70}$ verstehen. Devitt setzt aus produktionsästhetischer Perspektive Genre als ,,response to recurrent rhetorical situations ${ }^{\text {"71 }}$ an, die sowohl die semiotische Situation als auch den sozialen Kontext erfasst. Die Wahl des Genres ist dann sowohl ein Erkennen und Einordnen einer Situation als auch eine Form der adäquaten und effizienten Verarbeitung der Erfahrung dieser Situation. ${ }^{72}$ Burkhardt Meyer-Sickendieks Affektpoetik ergänzt dies um ein Verständnis der (literarischen) Gattungen als kulturelle Speichermedien, deren Adaption bei der Bildung ,emotionaler Kreativität‘ eine Rolle spielt und somit auch eine modellierende Wirkung auf die zugrunde liegenden erfahrenen Affekte hat, sowie Wissen von diesen Affekten transportiert. ${ }^{73}$ Zusammengenommen entspricht dieses Verständnis den Befunden aus den Forschungsmemoiren: Manches Mal erscheint dort die Wahl des Genres, in dem bestimmte Passagen gehalten sind, zunächst irritierend. Dieser Eindruck entsteht, weil diese Passagen meist affektiv negativ konnotierte Gegenstände und Situationen betreffen, über die die Forschenden keine Kontrolle haben und die bestimmte Textformen zu provozieren scheinen, welche in der Konstruktion der Forschungsmemoiren als eigene Gattung wiederum auffällig sind. Die Wahl der Textform dient der Einordnung und Verarbeitung von affektiv irritierenden Gegenständen in das eigene Forschungsnarrativ.

Dank eines (affekt-)poetologischen, weiten Formbegriffs lässt sich also, so die übergeordnete These für die nachfolgenden Kapitel, nachvollziehen, welche emotionale Textarbeit zu welchem Zweck an diverse stark affizierende ethologische Gegenstände angelegt wurde. Drei solche Gegenstände lassen sich exemplarisch anhand des Textkorpus untersuchen: Dies sind primatische Sexualität (Abschn. 3.2), primatische Gewalt (Abschn. 3.3) und primatisches Sterben (Abschn. 3.4). Alle drei verdienen bzw. erfordern eigene literarische Formen, die sich im wissenschaftlich-autobiographischen Bericht auf der Textoberfläche als Formveränderung - als (sub)kutane Irritation - bemerkbar machen und die ihrerseits auf Irritationen in der Affektlogik der methodisch kultivierten epistemologischen Emotionalität der primatologischen Feldforschung in ihrer zweiten historischen Periode hinweisen.

\footnotetext{
${ }^{69}$ Devitt: Generalizing about Genre, S. 573.

${ }^{70}$ Meyer-Sickendiek: Affektpoetik, S. 9.

${ }^{71}$ Devitt: Generalizing about Genre, S. 576.

${ }^{72}$ Siehe Devitt: Generalizing about Genre, S. 576.

${ }^{73}$ Vgl. Meyer-Sickendiek: Affektpoetik, S. 77.
} 


\title{
3.2 Vom Verhalten geschlechtsreifer Affen zu Zeiten der Feldbeobachtung: Das primatologische Melodrama
}

\subsubsection{Ethologische Schaulust}

\begin{abstract}
SEX APPEAL, that strange mystery, is a phenomenon as inexplicable and as obvious among chimpanzees, as human beings. (SM, 79)
\end{abstract}

,Sex‘ und ,Gender' nehmen als Konzepte und Gegenstände viel Raum in der Primatologie ein. Sie sind die Bausteine, aus denen größere Strukturen gebildet werden, welche man primatischen Gesellschaften zugrunde legen kann. So werden auch Formen menschlicher Sexualität und menschlicher Gesellschaften in diesem Zuge als Fortsetzung jener diskursiven Wissens-Explosion um den Sex herum, die Foucault als ,Scientia Sexualis ${ }^{674}$ fasst, mittels ethologischer Beobachtungen an Affen evolutionär verwurzelt. ${ }^{75}$ Zwar etablieren die ,Trimates" Goodall, Fossey und Galdikas (aber auch andere Forschende dieser Periode der primatologischen Feldforschung) einen Fokus auf weibliche Biographien und Biologien und können damit Fortpflanzungsprozesse und -verhalten unterschiedlicher Primaten zu vollen Narrativen von (weiblicher) Sexualität als Reproduktion formen. ${ }^{76}$ Das Vorhandensein von tierlicher Sexualität kann in der Forschung jedoch auch ein Problem sein, zunächst vor allem dort, wo sie vom ordnungsstiftenden Reproduktionsnarrativ abweicht. Biruté Galdikas verweist

\footnotetext{
${ }^{74}$ Vgl. Foucault, Michel: Der Wille zum Wissen. Sexualität und Wahrheit, Bd. 1. Frankfurt a. M. 1977 (frz. 1976), S. 55 ff.

${ }^{75}$ Bereits Solly Zuckermans Studie aus dem Jahr 1932 konzentriert sich vor allem auf eine systematisierende Untersuchung von Sexualität und Reproduktion. Vgl. SL, S. v-vi, siehe auch Abschn. 4.1. Fedigan widmet mit Primate Paradigms der Rolle von Sexualität im Paradigmenwechsel der Primatologie eine ganze Metastudie. Besonders zeigt sich eine Festschreibung der Gattung auf Basis von Analysen der Sexualität auch am Narrativ der Demonic Males, wie Richard Wrangham und Dale Peterson ihre populärwissenschaftliche Studie nennen. Diese formt basierend auf einem Studium der Berichte und Studien aus der Langzeitforschung eine Erzählung von der evolutionären primatischen männlichen Gewalt und damit zugleich eine Tragödie des Menschenmannes formt, der als Gewalt-Geschlecht seiner Genetik ausgeliefert auf den (unverschuldeten) ,Fehler' zuzulaufen droht (siehe Abschn. 3.3). Vgl. Wrangham, Richard W./Peterson, Dale: Demonic Males: Apes and the Origins of Human Violence. Boston 1996. Im Folgenden, wenn zur Unterscheidung nötig, als $D M$ geführt; Seitenangaben im Text.

${ }^{76}$ Diese Narrative halten als eigenständige Kapitel Einzug in die Forschungsmemoiren, z. B. als „Flo's Sex Life“ (SM, S. 79 ff.), „Flo and Her Family“ (SM, S. 101 ff.), „Mother and Child“ (SM, S. 225 ff.), „Mothers and Daughters“ (TW, S. 27 ff.), „Sex“ (TW, S. 72 ff.), „Sons and Mothers" (TW, S. 95 ff.), „Melissa“ (TW, S. 136 ff.) bei Goodall; „Beth“ (RE, S. 92 ff.), „Cara“ ( $R E$, S. 109 ff.), „Georgina“ (RE, S. 164 ff.), „Cara's Fate“ (RE, S. 235 ff.) bei Galdikas; „Peggy“ (AH, S. 38 ff.) bei Strum; sogar „Reproductive Features of the Limiting Sex“ in Hrdy, Sarah Blaffer: The Langurs of Abu. Female and Male Strategies of Reproduction. Cambridge, Mass./London 1977, S. 49 ff. Im Folgenden, wenn zur Unterscheidung nötig, als LA geführt; Seitenangaben im Text.
} 
an mehreren Stellen ihrer Forschungsmemoiren anekdotisch auf die sexuellen Handlungen von Affen (an Menschen), die außerhalb eines solchen Referenzrahmens liegen. So masturbieren gefangene Orang-Utans in Reflections of Eden unter Zuhilfenahme menschlicher Körperteile: „They inserted their little penises between her [Mrs. Alex's] fingers, first looking at her intently, then gazing off somewhere in their own private reverie. They were using her hands as tools, manipulating her fingers into positions for maximum pleasure" (137). Auch der schlüsselfigurhafte Orang-Utan Sugito verkompliziert das ödipale Dreieck, das Galdikas in Camp Leakey mit ihm und ihrem Mann pflegt (vgl. Abschn. 2.4.3), dadurch, dass er versucht, seinen Penis in Rod Brindamours Ohr einzuführen, und „also had tried to use Rod's hand to masturbate“ (130). Galdikas beschreibt diese Vorgänge nüchtern, legt aber Wert darauf, die affektiven Reaktionen der betroffenen Menschen festzuhalten. So verzeichnet Rods Gesicht „a look of disgust“ (130), Mrs. Alex zeigt „visible embarrassment“ (137) und „discomfort“, sowie, in der Suche nach einer Antwort auf die Frage „Are they perverted? Why do they do this?"“, ,a perplexed look“ (137). Dies sind jedoch Vorgänge, die Galdikas in diesem Sinne nicht selbst geschehen. Sie beobachtet, wie die Orang-Utans die anderen Menschen zu deren affektivem Unwohlsein instrumentalisieren, und dies - das sticht hervor - nicht zu Reproduktionszwecken, sondern in einer Formulierung der Anerkennung tierlicher Lust, zu Orang-Utan-,,pleasure" (137).

Auch Dian Fossey beobachtet in Gorillas in the Mist einen masturbierenden Gorilla und verweist auf tierlichen Lustgewinn. Sie sieht

Puck actively masturbating. His head was flexed backward, his eyes were closed, and he wore a semismile expression while using his right forefinger to manipulate his genital area. For about two minutes Puck appeared to be obtaining great pleasure from his actions before he stopped, self-groomed, and followed the others down the trail. (81)

Diese Szene folgt auf eine Interaktion zwischen diesem jungen Gorilla-Männchen Puck, Fossey und dem Silberrücken Beethoven. Pucks Selbstbefriedigung lässt sich also auch als durch Fossey inspiriert lesen. Sie selbst impliziert dies durchaus: „That Puck had apparently enjoyed the consequences of his actions [sie zu bedrohen und anzugreifen, sowie dadurch die Aufmerksamkeit eines Silberrückens $\mathrm{zu}$ erhalten] was obvious" und wertet die Masturbation im Sinne eines psychischen Mechanismus als ,,an unusual means of self-gratification“ (82).

Es geht mir im Folgenden jedoch um mehr als um tierliche Sexualität als Irritationsmoment für die normalerweise recht asexuelle, da entsubjektivierte Denkfigur , Tier ${ }^{6}{ }^{77}$ Aus literaturwissenschaftlicher Perspektive interessanter ist der der Verhaltensforschung eingeschriebene Akt der Beobachtung als Akt des Schauens, sowie seine Darstellung und Ausgestaltung im Bericht von der Feldforschung.

\footnotetext{
${ }^{77}$ Zur Funktion des Tieres als Wissens- und Denkfigur für den Menschen siehe Bühler, Benjamin/ Rieger, Stefan: Vom Übertier - Ein Bestiarium des Wissens. Frankfurt a. M. 2006, S. 9 ff.
} 
Der Akt der Beobachtung sexueller Handlungen ist im Bereich menschlichen Verhaltens deutlich und kategorial unterschieden von anderen möglichen Akten und Beobachtungen. Findet diese Beobachtung zwischen Menschen statt, so ist sie belegt mit Begriffen wie Voyeurismus und Pornografie und einem Affektspektrum von Scham bis zu Lust. Dabei wird die Schaulust häufig als „Modus“78 und - unter Rückführung auf die psychoanalytische Urszene der Beobachtung des elterlichen Geschlechtsaktes - als „Ursprung der Faszination“79 für jegliche Kulturrezeption betrachtet. Als solche muss sie vom Voyeurismus, der durch Anbindung an psychiatrische Diskurse pathologisierten Spezialform, und von der psychoanalytisch präzisierten Perversion abgegrenzt werden. ${ }^{80}$ Wird die Schaulust, ,als Grundelement der Sinnlichkeit“ als Indiz für den ,unauflösbaren Zusammenhang von Ästhetik, Erotik und Kreativität ${ }^{“ 81}$ betrachtet, so ist die Beobachtungssituation des Voyeurismus dagegen eine gewaltvolle. Denn, so Ulrich Stadler, die ,voyeuristische Person maßt sich eine Vorrangstellung an, indem sie sich das Recht auf Anonymität herausnimmt, das sie zugleich ihrem Gegenüber verweigert. “82 In diesem Sinn lässt sich das ethologische Beobachtungsverhältnis strukturell eher als voyeuristisch denn als ,nur' schaulustig lesen, wie folgende Passage aus Jane Goodalls In the Shadow of Man zeigt: ${ }^{83}$

The next day Flo arrived very early in the morning. Her suitors of the previous day [Goliath, David und Evered] were with her; once again they courted and mated with her before eating their bananas [...]. And then, from the corner of his eye, Hugo [van Lawick] saw another black shape in the bushes. As we peered we saw another, and another, and another. Quickly we withdrew into the tent and looked through binoculars into the vegetation. Almost immediately I recognized old Mr. McGregor. Then Mike and J.B. Also there were Huxley, Leakey, Hugh, Rodolf, Humphrey - and just about all the males I knew. [...] We remained inside the tent. Soon Flo moved up into the bushes and there was mated by every male in turn. ( 83 f., Hervorheb. MS)

\footnotetext{
${ }^{78}$ Stadler, Ulrich: Schaulust und Voyeurismus. Ein Abgrenzungsversuch. Mit einer Skizze zur Geschichte des verpönten Blicks in Literatur und Kunst. In: Ders./Karl Wagner (Hg.): Schaulust. München 2005, S. 9-37, hier S. 9.

${ }^{79}$ Roloff, Volker: Anmerkungen zum Begriff der Schaulust. In: Lydia Hartl/Yasmin Hoffmann/ Walburga Hülk/Volker Roloff (Hg.): Die Ästhetik des Voyeur. L'Esthétique du voyeur. Heidelberg 2003, S. 26-31, hier S. 26.

${ }^{80}$ Vgl. Stadler: Schaulust und Voyeurismus, S. 23; Roloff: Schaulust, S. 27 f.

${ }^{81}$ Roloff: Schaulust, S. 28.

${ }^{82}$ Stadler: Schaulust und Voyeurismus, S. 23.

${ }^{83}$ Inwiefern das (sexuelle) voyeuristische Verhältnis auch als ethologisches gelesen werden kann bzw. von seinen Akteur/innen als solches begriffen wird, zeigt der Fall des Voyeurs Gerald Foos, der ein Motel als Beobachtungsstation umrüstete, sich selbst als ,Sexualforscher ' bezeichnete und ein detaillierte Aufzeichnungen seiner Beobachtungen zur menschlichen Sexualität erstellte. Siehe Talese, Gay: The Voyeur's Motel. In: The New Yorker vom 11.04.2016, https://www.newyorker.com/magazine/2016/04/11/gay-talese-the-voyeurs-motel (16.05.2018).
} 
Eher zufällig begonnen (,from the corner of his eye“), wird das Schauen bei Goodall zur intensiven Tätigkeit (,,as we peered“). Je interessanter das ,Schauspiel' im sexuellen Sinn, desto weiter ziehen die beiden Beobachtenden sich hier in die Unsichtbarkeit (das Zelt) zurück. Sie verwenden aber zudem ein Medium, und zwar das Medium des Voyeurismus, das Fernglas, um das Geschehen genau beobachten und die daran Teilnehmenden identifizieren zu können: Goodall erkennt (,recognized“) alle Affen, ,all the males I knew“. Wie diese Passage jedoch auch zeigt, soll in Bezug auf die Beobachtung kopulierender Tiere durch ein menschliches Auge gerade ein emotionsloses Wahrnehmen, Beobachten, Aufzeichnen und Analysieren möglich sein, unter Ausschluss all jener Charakteristika der Aisthesis als (auch im Doppelsinne) sinnlicher Wahrnehmung. ${ }^{84}$ Nicht nur aber ist gerade der tierliche Körper von besonderer und medialer Faszination, wie Linda Williams in ihrer Studie zur Pornografie und der Lust am Schauen anhand der Konvergenz von der Lust an tierlicher Bewegung und jener an menschlichen Körpern in der Geburtsstunde des Films darlegt. ${ }^{85}$ Auch das Geschehen selbst kreiert einen affektiv bewertbaren Eindruck: Die Östrus-Zeit der Schimpansin Flo beschert Goodall und Ihrem Ehemann etliche neue Erkenntnisse und dokumentierbare neue Beobachtungen. Aber:

On the eight day of her swelling Flo arrived in camp with a torn and bleeding bottom. The injury must have just occured. [...] She looked somewhat tattered and exhausted by then and we were relieved for her sake that everything was over. [...] She looked worn out, faded, incredibly tattered after her strenuous five weeks. There were two extra pieces torn from her ears and a variety of cuts and scrapes all over her body. That day she just lay around camp for several hours, looking utterly exhausted. (87)

Die Schimpansin Flo erreicht, so stellt Goodall es hier dar, mit letzter Kraft das Camp und gleicht in ihrer körperlichen Verfassung dem Opfer eines besonders schweren sexuellen Übergriffs - und die einzige affektiv konnotierte Anmerkung, die sich Goodall in ihrem Bericht erlaubt, ist die, man sei ,for her sake" erleichtert, dass diese Zeit nun vorbei sei. Eine affektive Aisthesis in der Verhaltensforschung scheint zunächst im Rahmen des vorherrschenden Wissenschaftsethos undenkbar. Und doch drängt sich zumindest der Gedanke an das Problem der Schaulust und der kategorialen Bewertung obiger Szenen sowohl in Bezug auf seinen sexuellen wie den Rezeptionskontext auf. Wie bereits angerissen, wird in den Berichten der Feldforschung an Primaten häufig erstmals der Blick auf weibliche Primaten und deren Lebensläufe (,Life Stories') und Verhalten gerichtet. Gerade dort, wo die ,Life Story` zur Schilderung (der Beobachtung) eines sexuellen Aktes wird, tritt die Schaulust in Widerstreit mit dem Beobachteten und in der Folge in Form einer Irritation auf der Textoberfläche zutage, die nicht zuletzt Darstellungslust offenbart.

\footnotetext{
${ }^{84} \mathrm{Vgl}$. Roloff: Schaulust, S. 28.

${ }^{85}$ Vgl. Williams, Linda: Hard Core. Power, Pleasure, and the „Frenzy of the Visible“. Berkeley/ Los Angeles 1989, S. $34 \mathrm{ff}$.
} 
Die Passagen in den hier untersuchten Forschungsmemoiren fallen dadurch auf, dass ihre Teile auffallend miteinander streiten. Es sind meist Erzählungen davon, wie sich die Kopulationen gestalten, wenn eine weibliche Protagonistin in den Östrus kommt oder wie ein männlicher Protagonist seinen Reproduktionserfolg erlangt. Der Ton der Episoden ist vorgeblich nüchtern bis affirmativ, erzählerisch munter und er hebt seine Protagonist/innen durch Charakterisierungen und Wortwahl hervor. Inhaltlich aber wird dabei von Gruppensex über physische und psychische Nötigung bis zur Vergewaltigung kaum ein - nach menschlicher Norm- und Wertvorstellung - mehr oder minder problematischer Sexualakt ausgelassen. Diese werden aber nur selten so benannt.

Galdikas beobachtet z. B. in Reflections of Eden die Interaktion der OrangUtan-Jugendlichen Georgina mit dem subadulten Männchen BWC. BWC taucht im Geäst neben Georgina auf, die ihn ignoriert: „But then BWC grabbed her roughly by both legs and lifted up her bottom, which he began nuzzling with great interest. Clinging to a higher branch by her arms, Georgina squealed sharply then produced a hybrid whimper-squeal“ (172). BWC lässt Georgina daraufhin los und verschwindet; doch bald sieht Galdikas Georgina und BWC "traveling together" (172), denn ,,[a]lthough she had rejected him sexually, she did not reject him as a friend“" (172). Die Szene wirkt nun friedlich, die beiden Orang-Utans freundschaftlich und vertraut. Doch gerade als Galdikas in ihrem Text mit anderen Überlegungen befasst ist, geschieht Folgendes:

\begin{abstract}
BWC hastily constructed a nest and lay down. Only a few minutes later, he sat up and strode along a branch toward Georgina, low rumbles emanating from his throat. Georgina started whimpering. This time ignoring her protests, BWC wrapped his legs around Georgina's torso. She tried to push him away, but he brushed her hands aside and kept trying to position her with his legs. She continued to whimper and push him away. They grappled this way for about five minutes. BWC's movements were slow and deliberate. There was nothing rough. He acted as if he had all the time in the world. Finally, she was facing away, squeaking and breaking twigs. He was holding her by the legs, and adjusting himself under her. He pulled and pushed at her body until she was half facing him, but she kept twisting away. He finally achieved penetration, and pumped rhythmically for about a minute. But again, Georgina twisted away. (174)
\end{abstract}

Galdikas gestaltet diese Szene(n) genau aus, fasst nicht zusammen, sondern schildert jede einzelne Beobachtung bis hin zur Repetition. Im Anschluss gibt sie ihre Überlegungen zu Orang-Utan-Sexualität an: Diese „relationship“ habe ihr die Augen geöffnet (,opened my eyes“). Zuvor sei sie davon ausgegangen, ,that mating took place within the context of ongoing consortships between ,consenting adults““ (174). Nun sehe sie eine ,ambiguous, teenage quality to their relationship, as if they were just fooling around“ (174). Galdikas vergleicht das zuvor Geschilderte mit menschlichem Adoleszenz-Verhalten („Like many adolescent girls and boys“, 174), in dem die Partner/innen unterschiedliche Vorstellungen davon haben, was die Beziehung ausmacht: „Their last encounter resembled the stereotypical scene in which the teenage girl protests ,Don't, don't', while the teenage boy instists ,Why not, why not?““ (174). Dieser Verharmlosung des 
soeben Geschilderten - schließlich handelt es sich nicht um eine verbale, sondern um eine körperliche Auseinandersetzung - folgt dann jedoch eine deutliche Kategorisierung: „In effect, BWC had committed date rape“ (175, Hervorheb. MS). Wie um diese anthropomorphisierende Kategorisierung des Sexualaktes wieder abzuschwächen, folgt sogleich die Entschuldigung, man wolle nicht ,in any way [...] trivialize date rape among human beings" (175), aber:

[S]ex does not have the same meaning for orangutans that it does for humans. We view sex through cultural and moral lenses. For an orangutan, sex has no more meaning than eating. It is an expression of biological urges. An adolescent orangutan female may not enjoy forcible copulation, but neither does she suffer guilt and self-recrimination afterward. (175)

An dieser Aussage und ihren Zuschreibungen ist etliches auffallend, nicht zuletzt die Konzentration auf weibliche ,guilt“ und „self-recrimination“. Innerhalb des Textgefüges dieser Passage über Georgina und BWC reibt sich aber gerade die Darlegung der ,care-free'-Haltung der Orang-Utan-Sexualität mit der genauen Darstellung des Sexualkontakts und des Abwehr- und Verteidigungsverhaltens des Orang-Utan-Weibchens. Die Zuspitzung der Erzählung hin zum Begriff „date rape“ und die dezidierte Korrelation mit menschlichen Altersgenoss/innen kollidiert mit der Antiklimax der Abschwächung derartiger Akte in der OrangUtan-Sexualität. Deren Botschaft (,Bloß nicht anthropomorphisieren!') steht im Widerspruch sowohl zu der vorangegangenen Darstellung als auch zu den Mitteln der Argumentation, die sehr deutlich vorgeben, genau zu wissen, was Orang-Utans denken und fühlen (nämlich wohl: nichts).

Sowohl Goodalls als auch Galdikas’ Passagen präsentieren hier Vorgänge, die einen Leseeindruck generieren, der im Gegensatz zur expliziten Botschaft - so vielfältig und aufschlussreich, aber von uns nicht moralisch zu bewerten ist primatische, nicht-menschliche Sexualität - steht. Mehr noch aber: In der Schilderung sexueller Affenbegegnungen wird die Erzählung vom Forschen auch textformal auffällig, denn sie wird melodramatisch ausgestaltet.

\subsubsection{Primatologie im melodramatischen Modus}

Es soll nun nicht behauptet werden, die primatologischen Texte würden Melodramen mit Affenbesetzung aufführen. ${ }^{86}$ Es soll auch nicht eine schlichte Analogie hergestellt werden zwischen den häufig weiblichen primatischen ,Life Stories“ und der (historischen) größtenteils filmwissenschaftlichen Einordnung

\footnotetext{
${ }^{86}$ Vinzenz Hediger sieht jedoch zu Recht die primatologische Wissensvermittlung gerade im Dokumentarfilm bei Jane Goodall „durch die Form eines romanhaften Dramas“ vollzogen, „,das zugleich als reflexive Schule des Fühlens und der Anteilnahme strukturiert ist". Hediger: Was will Jane im Dschungel?, S. 58.
} 
des Melodrams als Genre des Femininen. ${ }^{87}$ Der melodramatische Modus, wie ihn die Forschung konzipiert, lässt sich stattdessen als analytisches Konzept heranziehen, um die Form bestimmter Passagen in diesen Texten, die sich um beobachtete Sexualität drehen, und ihre Rolle im Kontext primatologischer Emotionalität zu untersuchen. ${ }^{88}$

\subsubsection{Die ästhetische Form Melodrama}

Wie bereits der Theaterwissenschaftler Eric Bentley ausführt, bedeutet etwas als ,dramatisch" wahrzunehmen, ,both to perceive elements of conflict and to respond emotionally to these elements of conflict " ${ }^{89}$ Reale Ereignisse selbst sind für Bentley nicht an sich dramatisch. Sie können jedoch Elemente des ,Dramas' beinhalten, welche mithilfe von Narrativ - darunter versteht Bentley „suspense“ und „the arrangement of incidents in chronological order" ${ }^{\circ 90}$ - und Plot, ,,which is narrative with something ,done to it “", nämlich ,a rearrangement of the incidents in the order most calculated to have the right effect", 91 zutage gefördert werden können. Dass auch Forschungstexte die berichteten Ereignisse chronologisch und dramaturgisch anordnen und Gebrauch von narrativen Strukturen machen, dürfte augenfällig sein. ${ }^{92}$ Dass die hier betrachteten primatologischen Forschungsmemoiren auch auf das (melodramatische) Plot-Element zurückgreifen, soll im Folgenden aufgezeigt werden.

Peter Brooks' einflussreiche Studie zur melodramatischen Imagination vertieft Bentleys Denklinie und sieht das Melodrama - ganz im Sinne von Amy Devitts Genre-Konzeption - als ,a form of conception and expression“, ,a certain fictional system for making sense of experience, as a semantic field of force“, gar als ,a

\footnotetext{
${ }^{87}$ Siehe zum Kontext dieser Zuschreibung und ihres Wandels Williams, Linda: Melodrama Revisited. In: Nick Browne (Hg.): Refiguring American Film Genres. Theory and History. Berkeley/Los Angeles 1998, S. 42-88.

${ }^{88}$ Dass das primatologische Darstellen und Erzählen von einer anglo-amerikanischen Vorliebe für das Melodramatische geprägt ist, zeigt sich allein schon an Überschriften wie „Larder, Nursery and Seraglio" ( $L A$, S. $91 \mathrm{ff}$.) in der sich von der Tradition der ,Trimates" stilistisch und forschungsethisch distanzierenden Schrift Hrdys oder „Capture and Release“ (AH, S. 229 ff.) und „Desperation - and a Happy Ending“ (AH, S. 218 ff.) bei Shirley C. Strum.

${ }^{89}$ Bentley, Eric: The Life of the Drama. London 1965, S. 4, Hervorheb. im Original.

${ }^{90}$ Bentley: The Life of the Drama, S. 14.

${ }^{91}$ Bentley: The Life of the Drama, S. $14 \mathrm{f}$.

${ }^{92}$ Mit Hayden White, der sich auf Northrop Fryes Gattungstheorie bezieht, wäre hier die Rede von der Organisation der zu erörterten Ereignisse (der ethologischen Daten) in zeitlicher Reihenfolge (,chronicle'), der Aufbereitung der Ereignisse in einem dramaturgisch strukturierten ,Schauspiel' (,spectacle'; dieses produziert bei White die ,story') und der narrativen Strukturierung mittels gattungstypischer motivischer Verschlüsselung und Erzählstrategien. Vgl. White, Hayden: Metahistory. The Historical Imagination in Nineteenth-Century Europe. Baltimore/London 1973, S. 5 f. Siehe auch Myers: Writing Biology, S. 35.
} 
sense-making system“ “93 In Hinblick auf den hier propagierten Konnex von affektiver und Textform-Irritation ist es besonders aufschlussreich, dass Brooks seine Ideen zum melodramatischen Modus aus der Literatur (und nicht dem Theater oder Film) ableitet. Es sind die Romane Henry James' und Honoré de Balzacs, in denen ,we can observe the narrator pressuring the surface of reality (the surface of his text) in order to make it yield the full, true terms of his story. "94 Der melodramatische Modus presst, wenn man Brooks und Bentley hier folgen mag, den realen Ereignissen durch diverse kutane und subkutane (literarische) Verfahren der An- und Umordnung, der Explizitmachung und Typisierung sowie auch der Polarisierung zugespitzte Bedeutung ab, ja lässt eine Form - ,,a certain imaginative complex and a set of dramatic conventions“95 - entstehen, in der diese Bedeutung vermittelbar und ablesbar wird. ${ }^{96}$ Als ästhetische Form ist der melodramatische Modus wie andere Ausdrucks- und Repräsentationsmodi „means for interpreting and making sense of experience. “97

Brooks und Bentley sehen die (melo-)dramatische Form somit als zutiefst menschliches Charakteristikum, als Verfahren des homo significans ${ }^{98}$, um Erfahrungen und Erlebnisse vorstellbar und interpretierbar zu machen. Robert Heilman spricht in ähnlicher Linie davon, Tragödie und Melodrama seien, Versionen' von Erfahrungen, eine Art Perspektive, unter der das Material (die Ereignisse) mittels , a certain generic conformation“99 $\mathrm{zu}$ einer bestimmten Erfahrung geordnet werde. Dabei besteht für Heilman zwischen literarischer Imagination und ,realer' Erfahrungskonzeption nicht nur eine Analogie, sondern auch eine Interaktion:

Both dramatists and ordinary men set forth versions of experience, and there are analogies between popular attitudes and literary formulations. What is more, they interact [....]. In ordinary life we frequently act, think, and feel as if we were participating in a tragedy or melodrama, or as if our experience had taken on one generic form or another. ${ }^{100}$

\footnotetext{
${ }^{93}$ Brooks, Peter: The Melodramtic Imagination. Balzac, Henry James, Melodrama, and the Mode of Excess. Yale/New Haven 1995, S. xvii.

${ }^{94}$ Brooks: The Melodramatic Imagination, S. 1 f.

${ }^{95}$ Brooks: The Melodramatic Imagination, S. 202.

${ }^{96}$ Vgl. Brooks' Charakterisierung von Balzacs und James' Prämisse für die Darstellung von Welt in ihrer Literatur: „, [T] he surface of the world - the surface of manners, the signifiers of the text are indices pointing to hidden forces and truths, latent signifieds“. Brooks: The Melodramatic Imagination, S. 199.

${ }^{97}$ Bentley: The Life of the Drama, S. 206.

${ }^{98}$ Vgl. Brooks: The Melodramatic Imagination, S. 206; Bentley: The Life of the Drama, S. 216.

${ }^{99}$ Heilman, Robert Bechtold: Tragedy and Melodrama. Versions of Experience. Seattle/London 1968, S. ix.

${ }^{100}$ Heilman: Tragedy and Melodrama, S. ix.
} 


\subsubsection{Melodramatische Motive, moralisches Residuum und Gefühlsarbeit}

In der Forschungsliteratur zum Melodrama ist viel vom „mode of excess“"101 und Tendenzen zu einer ,inflated and sententious"102 Rhetorik die Rede - Züge, die selbst der Sentimentalismus-Vorwurf nur bedingt an die Forschungsmemoiren der Primatologie herantragen würde. ${ }^{103}$ Der melodramatische Modus zeichnet sich jedoch durch einige Charakteristika aus, die treffend die Verfahren beschreiben, mit denen diese Texte von herausfordernden Szenarien und Erfahrungen berichten. ${ }^{104}$ Dies sind erstens melodramatische Motive auf der Plot-Ebene, wie Heilman sie zusammenfasst als ,pursuit and capture, imprisonment and escape, false accusation, cold-blooded villain, innocence beleaguered, virtue triumphant, eternal fidelity, mysterious identity, lovers reconciled, fraudulence revealed, threats survived, enemies foiled“105 sowie der nur teilweise ins Symbolische überführte Widerstreit zwischen femininer Liebessuche und maskulinem Sexualitätsdrang als „Instanzen sentimentaler Phantasie“ in einem „Antagonismus von bedrohlicher Sexualität und zivilisierender Liebe“, ${ }^{106}$ wie Hermann Kappelhoff dies markiert. Liest man z. B. Jane Goodalls Beschreibungen der Östrus-Zeiten bei den Schimpansen, so finden sich diese Motive leicht darin wieder: In ihren zweiten Forschungsmemoiren Through a Window wird recht schnell zu Beginn des Kapitels „Sex“ (72 ff.) der jüngst aus seiner Stammgruppe vertriebene Schimpanse Evered als Protagonist installiert. Er wird von einem Mitarbeiter Goodalls im Gebüsch entdeckt, in der Begleitung eines Weibchens im Östrus: „Here was no lonely exile!“", heißt es triumphierend, und weiter: „Not only was Evered in the company of a female, but a highly desirable female, at the height of her sexual receptivity“ (72). Evereds Status als Held einer Vertreibungs- und Eroberungserzählung wird durch die Frage „How many such sexual dalliances had Evered enjoyed during the months that he was driven from his community [...]?“(72) noch unterstrichen. Nicht nur fällt die seltsam viktorianisch

\footnotetext{
${ }^{101}$ Brooks: The Melodramatic Imagination, S. xiii.

${ }^{102}$ Brooks: The Melodramatic Imagination, S. 40.

${ }^{103}$ Vgl. zur Geschichte und Entwicklung dieser Abwertung in der Filmwissenschaft Williams: Melodrama Revisited, S. 43 f.

${ }^{104}$ Dass der melodramatische Modus auch herangezogen werden kann, um zu untersuchen, wie die primatologischen Texte Emotionen erzeugen, soll an dieser Stelle vorausgesetzt, aber zu Gunsten seiner poetologischen, produktionsästhetischen Funktion nicht weiter untersucht werden. Gerade aus filmwissenschaftlicher Perspektive liegt der Fokus überwiegend rezeptionsästhetisch und historisch und an theatertheoretische Überlegungen von Aristoteles bis Lessing anknüpfend auf dieser emotionserzeugenden Kraft des Melodramas. Vgl. Williams: Melodrama Revisited, S. 44, oder Kappelhoff, Hermann: Matrix der Gefühle. Das Kino, das Melodrama und das Theater der Empfindsamkeit. Ber$\operatorname{lin} 2004$.

${ }^{105}$ Heilman: Tragedy and Melodrama, S. 76.

${ }^{106}$ Kappelhoff: Matrix der Gefühle, S. 233. Kappelhoff erweitert diesen Grundkonflikt um die Umkehrung jener Geschlechterrollen in die (über-)sexualisierte Frau und den lichten, nämlich tugendhaften Helden - eine Figurenkonstellation, die sich auch in den primatologischen Texten häufig dort zeigt, wo adoleszente, gerade in den ersten Östrus getretene Weibchen die Aufmerksamkeit eines älteren Alpha-Männchens auf sich zu ziehen versuchen und von diesem vorbildhaft bis desinteressiert zurückgewiesen werden.
} 
anmutende Wortwahl „,sexual dalliances“ auf; bald danach endet auch „Evered's persecution“ (73, Hervorheb. MS) durch den Alpha-Schimpansen, und Evered „,was able to return and take up his position in the Kasakela community“ (73, Hervorheb. MS). Dort kann er seine ,periodic romantic adventures“" weiter verfolgen und den beobachtenden Forschenden seine „consummate skill[s]“ und „techniques“ (73) zeigen, später ist sogar die Rede von „Ploys“ (78). Diese sind „,coercing females to follow him" und ,preventing their escape before he has a chance to impregnate them“ (73). Goodall bezeichnet dies als ,short-and-sweet method“ (78). Eine andere ,Methode“ ist die Entführung eines Weibchens für einen ganzen Zyklus mit folgendem Vorteil: „He can mate her whenever he likes, without fear of interruptions by his superiors“ (78). Beides sind Strategien, sich nicht den Zugang zum weiblichen Genital mit anderen Männchen teilen zu müssen. Als Beispiel für Evereds Talente in diesem Wettstreit wird seine Anbandelung mit Winkle geschildert:

\begin{abstract}
It began one morning when Evered came upon Winkle and her son Wilkie [...]. She was just beginning a sexual swelling, and Evered was immediately interested, examining her posterior carefully, then sniffing his finger. [...] After ten minutes Evered moved away, then turned, and staring at Winkle, began, with quick jerky movements, to shake a leafy branch. Roughly translated this meant: ,Come, follow me!' (If the branch shaking is accompanied by a penile erection it means ,Come here! I want to copulate with you."). [...] Over the next twenty minutes, Evered kept on repeating his summons and, as Winkle continued to ignore him, his shaking of the vegetation became more and more violent. It was obvious that his patience was gradually wearing thin, and finally he gave out altogether. With hair bristling, lips compressed, he leapt onto Winkle, pounding and dragging her until she pulled free and ran off screaming. Evered, panting from his exertions, once more summoned her, but still she refused to obey. She just sat looking at him, her screams gradually giving place to little squeaks, then whimpers. (73 f.)
\end{abstract}

Evered bleibt auch weiterhin in Goodalls Schilderung der ethologisch les- und übersetzbare Protagonist und Fokus dieser Szene, so ist z. B. seine ,patience [...] quite remarkable“ (74). Weitere Schläge bescheren ihm Erfolg:

Now at last, when he stopped his pounding and summoned her to approach she responded instantly. Hastening to crouch before him, with nervous panting grunts, she pressed her mouth to his thigh, kissing him. And then, as is the way of male chimpanzees after aggression, Evered reassured her, grooming her until she relaxed under the gentle caress of his fingers. Once punishment has been handed out, then it is time to make amends, to restore social harmony. (74)

Auch hier fällt die Zusammenstellung von lustvollem Darstellungsreichtum der ausgeübten Gewalt und harmonisierenden Einordnungen in ein durchweg mit anthropomorphisierendem Vokabular markiertes sozialphilosophisches Schema (,punishment", ,amends', ,social harmony') auf. Diese Zusammenstellung leitet eine melodramatische Formulierung weiblicher ,victimhood“ ein: ${ }^{107}$ „Wilkie

\footnotetext{
${ }^{107}$ Zur subjektkonstituierenden Funktion der Anerkennung von und Identifizierung mit ,female suffering ' und ,victimhood' im klassischen Melodrama und dessen Rezeption siehe Williams: Melodrama Revisited, S. 47 f. und Williams, Linda: Film Bodies: Gender, Genre, and Excess. In: Film Quarterly 44/4 (1991), S. 2-13.
} 
[Winkles Sohn] shared his mother's nest as usual, and surely the contact with his small, familiar body gave her some comfort after the bruisings and batterings of the long day“ (75). Dieser Satz sticht umso mehr hervor, als in der Folge weiterhin Evered als Fokus dieser Erzählung eines Reproduktionserfolgs dient. In der Abgeschiedenheit kann Evered seine milde Seite zeigen (,safe from discovery [...] Evered became benign and tolerant“ 75); er begattet Winkle „seldom - never more than five times in one day“ (75). Alles hier scheint für Goodall wohl eingerichtet: „It was all so peaceful, like some idyllic honeymoon“ (75). Um das Drama einer Entführung und Nötigung als romantische Erzählung abzurunden, wird schließlich ein Kind geboren: ,eight months after Winkle returned from her honeymoon with Evered she gave birth to a daughter" (76). ${ }^{108}$ Die Ereignisse des reproduktiven Geschehens werden so nicht nur im Narrativ angeordnet, sondern auch als Plot zu einem bestimmten Effekt - dem lobenswerten Reproduktionserfolg - hin strukturiert und ge- bzw. überformt.

Auffallend ist dabei weiter, wie gegensätzliche Topoi im szenischen Bericht von einem reproduktiven Verhalten miteinander vereinbart werden müssen. Einerseits ist Evered der bewundernswerte Held einer Erzählung, die ihn aus der Ausgestoßenheit und dem sicheren Niedergang zu einem der reproduktiv erfolgreichsten Schimpansenmännchen werden lässt. Sex wird von Goodall abschließend sogar ausschließlich in diese Richtung gelesen: ,[H]e will have a good chance, in this peaceful setting, of siring a child, propagating his genes, - which, after all, is what sex is all about“ (78). Andererseits schildert Goodall hier deutlich Szenen einer gewalttätigen Beziehung, in denen die weibliche Figur Winkle in eine klassische Opferrolle gedrängt wird. Goodall erkennt dies auch sichtlich, wenn sie einräumt: „Even though the female is seldom the victim [of group aggression during mating], the situation clearly subjects her to a certain amount of stress" (76, Hervorheb. MS). Eine derartige Text-Irritation kulminiert häufig sogar innerhalb eines Satzes, so wenn Goodall schreibt: „The aggressive bullying ceases once the male has achieved his goal, and he is then prepared to adjust his daily routine to that of his lady" (75, Hervorheb. MS). Der erste Teilsatz scheint dem ethologischen Beschreibungskodex $\mathrm{zu}$ entsprechen (das Verhalten „ceases once the male has achieved his goal“), das Verhalten selbst wird aber als ,aggressive bullying“ bereits zur anthropomorphen Kategorie pathologischer Formen. Der zweite Teilsatz fügt dann eine Vermutung über den tierlichen Geisteszustand und Willen (,he is then prepared to adjust“) mit einer ironisch der Hohen Minne verschriebenen Vokabel zusammen (,his lady“).

Hier scheint sich abzubilden, was Christof Decker als „Dialektik von Pathos und Aktion“109 im melodramatischen Modus bezeichnet. Dieser Darstellungsstil

\footnotetext{
${ }^{108}$ Hier erinnert die erzählerische Einhegung motivisch an Heinrich von Kleists Novelle Die Marquise von $O \ldots$, auf deren narrative Doppelstruktur schon verschiedentlich hingewiesen wurde, siehe z. B. Kotin Mortimer, Armine: The Devious Second Story in Kleist's Die Marquise von O.... In: The German Quarterly 67/3 (1994), S. 293-303.

${ }^{109}$ Decker, Christof: Hollywoods kritischer Blick. Das soziale Melodrama in der amerikanischen Kultur 1840-1950. Frankfurt a. M./New York 2003, S. 9; in Rückgriff auf Williams: Melodrama Revisited, S. 42.
} 
ist besonders dort wichtig, wo ,[i]ntensive Gefühle und mitreißende Handlungen [...] in einen Reflexionsprozeß einbezogen werden“, 110 um kontroverse Themen aufzunehmen und bestimmte Anliegen zu verbreiten. Unterschiedliche Affektqualitäten wie „Mitleid, Mitgefühl, Sympathie, Schock oder Anklage“ tragen dazu bei, „daß Wertkonflikte ausagiert werden, für die mit fiktionalen Mitteln nach neuen Wahrnehmungs- und Verhaltensmustern gesucht wird“. ${ }^{111}$

Ein weiteres Charakteristikum des melodramatischen Modus ist also zweitens das moralische Residuum. Mit diesem Begriff bezieht sich Decker auf Peter Brooks' Konzept des moralisch Okkulten. ${ }^{112}$ Brooks spielt in seiner Untersuchung des melodramatischen Modus mit dem Bild einer Sitten- und Textoberfläche, der subkutan verborgene und daher, okkulte' Bedeutung abgepresst werden kann. Er bezieht sich dabei wiederum auf I. A. Richards Metapherndefinition, ${ }^{113}$ die in Brooks' Ausführung eine Metapher zur geradezu osmotischen Transaktion zwischen Kontexten werden lässt: ,[P] ressure on the primary context is such that things and gestures are made to release occult meanings, to transfer significance into another context". ${ }^{114}$ Der melodramatische Modus ist daher bei Brooks insofern metaphorisch strukturiert, als ,to the melodramatic imagination, significant things and gestures are necessarily metaphoric in nature because they must refer to and speak of something else". ${ }^{115}$ Was dieses , something else" sein könnte, ergibt sich aus der Genese des Melodramas: Decker wie Brooks leiten seine Entstehung aus einem historisch-politischen Kontext ab und untersuchen die Instrumentalisierung des Melodramas als Vermittlungsorgan moralisch-ethischer Werte und eines Wissens darum - eines tradierten ,Wertefundus', der durch politische und epistemische Umwälzungen erschüttert wurde, aber gerade auf emotionaler Ebene weiterwirkt. ${ }^{116}$

\footnotetext{
${ }^{110}$ Decker: Hollywoods kritischer Blick, S. 9.

${ }^{111}$ Decker: Hollywoods kritischer Blick, S. 11. Ähnlich verfährt die sentimentale Literatur des 19. Jahrhunderts, die sich vergleichbarer Abwertung ausgesetzt sah wie das Melodrama im 20. Jahrhundert. Emotionalität wird für ethische Anliegen instrumentalisiert, um „Gefühle zu erzeugen, um Handlungen oder Ideen zu beeinflussen und moralische Korrekturen vorzunehmen“. Decker: Hollywoods kritischer Blick, S. 11. Siehe auch Tompkins, Jane: Sensational Designs. The Cultural Work of American Fiction 1790-1860. New York/Oxford 1985, S. 122 ff.

${ }^{112} \mathrm{Vgl}$. Brooks: The Melodramatic Imagination, S. 5.

${ }^{113}$ Brooks verweist auf Richards, Ivor Armstrong: The Philosophy of Rhetoric, New York 1936, S. 94.

${ }^{114}$ Brooks: The Melodramatic Imagination, S. 9.

${ }^{115}$ Brooks: The Melodramatic Imagination, S. 9 f.

${ }^{116}$ Decker sieht ähnlich wie Brooks das Melodrama als instrumental für die Verbreitung demokratischer Kultur. Bei Brooks betrifft dies die Genese des melodramatischen Modus aus den Umwälzungen der Französischen Revolution, bei Decker im Rahmen der Reformästhetik des Kinos die Modellierung demokratischer Gefühlsstrukturen vor allem im Zusammenhang amerikanischer Populärkultur. Vgl. Brooks: The Melodramatic Imagination, S. 14 ff.; Decker: Hollywoods kritischer Blick, S. 9 ff.
} 
Für beide findet im Melodrama daher vor allem drittens eine Gefühlsarbeit statt. Das moralische Residuum stellt, so Decker mit Raymond Williams, ${ }^{117}$

eine spezifische Gefühlsstruktur dar, die sich in der Vergangenheit herausgebildet hat und Erfahrungen, Werte oder Sinndimensionen umfaßt, für die es innerhalb der dominanten Kultur keinen entsprechenden Ausdruck mehr gibt - auch wenn sie teilweise in diese eingegangen sind. Was sich dahinter verbirgt, bleibt so lange offen, bis das Melodrama zu seiner Aufdeckung und Entzifferung beiträgt. ${ }^{118}$

Melodramatische Formen gewinnen kulturellen Sinn erst aus einer solchen Arbeit am moralischen Residuum, ,jener Gefühlsstruktur, die Restbestände anachronistisch gewordener Bindungskräfte mit neuen Erfahrungsgehalten zusammenführt." "119

Hier lässt sich die primatologische Verhaltensforschung analog setzten: In ihr drängen kulturell bedingte Erzählkonventionen, anthropozentrisches Vorstellungsvermögen und anthropogene Moralvorstellungen gegen das Reglement neuer (wissenschaftlicher) Ordnungen, in denen das Tier als evolutionäre Black Box und Verhalten als arterhaltend, ökologisch oder reproduktiv ableitbar und daher unbedingt sinnvoll angesehen wird. Der affektive Tumult der Beobachtung einer Szene gewalttätiger Sexualität trifft auf die ethologische Prämisse, als regelhaftes Verhalten müsse diese Form der Sexualität evolutionär sinnvoll und (in Hinblick auf den Reproduktionserfolg) zweckerfüllend sein.

Gefühlsarbeit muss in den primatologischen Berichten jedoch auch im arbeitssoziologischen Sinn verstanden werden, wie ihn Hochschild definiert hat als Arbeit an der eigenen Emotionalität im Arbeitsfeld, „damit die äußere Haltung gewahrt bleibt, die bei anderen die erwünschte Wirkung hat". ${ }^{120}$ Hochschild betont in ihrer Studie zur Gefühlsarbeit die indikatorische Funktion (,Signalfunktion") von Gefühlen: Gefühle sind hier Ausdruck innerer Einstellungen, die die Wahrnehmung beeinflussen, wenn nicht sogar steuern. Emotionalität fungiert für Hochschild als „Orientierungsrahmen für die persönliche Bedeutung“ von „Wahrnehmungen, Erinnerungen oder Vorstellungen“: „,[d]ie Gefühlswelt gibt dem Betrachter einen Standpunkt" ${ }^{121}$ In den hier besprochenen Passagen stößt ein solcher individuell affektiv konstituierter Orientierungsrahmen für die Bewertung des Beobachteten auf die normative Vorgabe einer Wertneutralität und evolutionärer Funktionalität allen beobachteten Verhaltens. Im melodramatischen

\footnotetext{
${ }^{117}$ Williams, Raymond: Marxism and Literature, Oxford: Oxford University Press 1977, S. $121 \mathrm{ff}$.

${ }^{118}$ Decker: Hollywoods kritischer Blick, S. 36.

${ }^{119}$ Decker: Hollywoods kritischer Blick, S. 39.

${ }^{120}$ Hochschild, Arlie Russell: Das gekaufte Herz. Zur Kommerzialisierung der Gefühle. Frankfurt a. M./New York 1990 (engl. 1983), S. 29.

${ }^{121}$ Hochschild: Das gekaufte Herz, S. 48.
} 
Modus findet diese durch den Beruf der Feldforschenden geforderte Gefühlsarbeit ein Instrument, dialektisch ausagiert zu werden - und dabei irritative Textphänoneme zu erzeugen, die wieder indikatorisch auf diese Gefühlsarbeit verweisen.

\subsubsection{Die Lesbarkeit von Affen und Affekten}

Der melodramatische Modus ist nicht nur Austragungsmedium dieser Gefühlsarbeit, sondern stellt auch eine Art affektiver Erleichterung zur Verfügung, indem er die stimulierende Kanalisierung emotionaler Ambivalenz oder Pluralität anbietet. Heilman bietet hierfür den Begriff monopathy an. ${ }^{122}$ Im kulturellen Kontext ist dies eine Intensivierung durch Konzentration: Die ungeteilte Aufmerksamkeit auf dem Erleben nur einer emotionalen Dimension steigert den ,Genuss' der affektiven Erfahrung. Weil melodramatische Figuren in gewisser Weise eindimensional sind, müssen wir nicht mit widersprüchlichen Emotionen in ihrer Einschätzung und Bewertung kämpfen, sondern können sie affektiv einheitlich wahrnehmen: ${ }^{123},[$, [ ] n monopathy we are spared all contradictions and contingencies“"124. Durch diese Reduktion, bzw. Konzentration des vorherrschenden Gefühls ermöglicht der melodramatische Modus mittels einer simulierten ,wholeness ‘ oder , oneness ' Urteils- und Handlungsfähigkeit, ${ }^{125}$ die auch für die primatologischen Beobachtenden und Aufzeichnenden zum Tragen kommt. Diese monopathy ist das Angebot des melodramatischen Modus an

\footnotetext{
${ }^{122}$ Heilman: Tragedy and Melodrama, S. 96.

${ }^{123}$ Heilmans Konzeption des Melodramatischen muss komplementär zum Tragischen verstanden werden: Das Tragische bietet den Raum für widerstrebende affektive Bewegungen, es ist inklusiver und zugleich macht es den Exzess erfahrbar. Das Melodramatische dagegen bietet zum Beispiel ,the joy of conquest“ ohne die realen Komplemente dieser Emotion, „,sense of exhaustion, fear of injustice to others, shock at our own ruthlessness, disillusionment with the ends achieved“. Heilmans Modell von Gefühlen konzipiert diese also als vielschichtige und eigentlich (real) unisolierbare Phänomene: „An incomplete emotion is one which mirrors only part of the actual or possible human engagement in the given circumstances". Heilman: Tragedy and Melodrama, S. 96.

${ }^{124}$ Heilman: Tragedy and Melodrama, S. 95.

${ }^{125}$ Heilman entwirft hier ein affektives Handlungsmodell, das in der Reduktion und Konzentration auf Einheitlichkeit besteht: „In the structure of melodrama, man is essentially ,whole“; this key word implies [...] an absence of the basic inner conflict that, if it is present, must inevitably claim our primary attention. Melodrama accepts wholeness without question [...]. He [man] is not troubled by motives that would distract him from the outer struggle in which he is engaged. He may indeed be humanly incomplete; but his incompleteness is not the issue. It is in tragedy that man is divided; in melodrama, his troubles, though they may reflect some weakness or inadequacy, do not arise from the urgency of unreconciled impulses“. Heilman: Tragedy and Melodrama, S. 79. ,,[M] an has to assume wholeness to act in the world. He cannot act if he is beset by guilt or by conflicting impulses that make choice exhausting or impossible [...]. Wholeness $[\ldots]$ is morally neutral; it is an artificial unity - the simple condition of action with or without others" (S. 98).
} 
eine Forschungsform, die Empathie instrumentalisiert und Situationen begegnet, in denen gerade diese kultivierte Empathie das ,neutrale " Forschungssubjekt als erzählendes und als bewertendes Ich bedroht, indem sie potenziell vielfältige Gefühle produziert. ,[T]o perceive elements of conflict and to respond emotionally to these elements of conflict", ist Bentleys Beschreibung der dramatischen Wahrnehmungsweise. Sie trifft deutlich zu auf die Art und Weise, wie menschliche Beobachtende sexuelle Akte und Verhaltensweisen unter Affen wahrnehmen, diesen Tieren, die als menschenähnlich betrachtet werden können und dennoch außerhalb menschlicher Norm- und Wertesysteme stehen sollen. Zusammen mit der melodramatischen Bedeutungskonstitution, die übertragen in die primatologische Epistemologie aus jeder Geste, aus jedem Verhalten einen okkult-evolutionären Sinn liest, ermöglicht sie eine Lesbarkeit von Körpern und eine Verarbeitung von konfliktiver Emotionalität, die wesentlich auf moralischen Vorstellungen beruht.

Der melodramatische Modus fügt sich jedoch vor allem gut in primatologische Erzählformen, weil er seine Figuren auf „ever more concentrated and totally expressive gestures and statements" ${ }^{126}$ und auf Stereotypen der Familienaufstellung reduziert. In der Primatologie geht es - auch in ihrer empathischen Form - ebenfalls nicht darum, individuelle Charaktere zu identifizieren, sondern anhand von Individuen generelle, modellhafte Aussagen über die Gattung und ihre Exemplare treffen zu können. „In melodrama we accept the part for the whole; this is a convention of the form", ${ }^{127}$ wie Heilman schreibt. In der Ethologie steht das Individuum für die Gattung, und sein Verhalten metaphorisch für evolutionäre Zusammenhänge oder kognitives Vermögen: auch hier Konventionen der (Forschungs-)Form. Wenn Stereotypen im Bereich der melodramatischen Fiktion als „cultural shorthand“ 128 enorme Mengen kultureller Informationen in extrem kondensierter Form sowie emotionale Assoziationen transportieren können, wie Jane Tompkins für die ,Sentimental Novel' festhält, so lassen sich Affen in den primatologischen Berichten leicht äquivalent als figurale Verdichtungen in einer Erzählung von der Evolution begreifen.

Gerade als äffisches ist das Personal der Primatologie zudem ideal für die melodramatische Imagination: Wie Brooks zuspitzt, werden im Melodrama ,[s]ubjects [...] evidently conceived for their plastic figurability, the dramatic interplay of posture and gesture. The spoken word is rarely used toward the formulation of significant messages; it is largely confined to emotional utterances, outburst, expressive cadenzas." ${ }^{29}$ Der melodramatische Modus steht explizit in Zusammenhang mit der Geste in der Sprachphilosophie und der Pantomime als

\footnotetext{
${ }^{126}$ Brooks: The Melodramatic Imagination, S. 4.

${ }^{127}$ Heilman: Tragedy and Melodrama, S. 79.

${ }^{128}$ Tompkins: Sensational Designs, S. xvi.

${ }^{129}$ Brooks: The Melodramatic Imagination, S. 65.
} 
theatrale Ausdrucksform, ${ }^{130}$ einer körperlichen Beredsamkeit in der Tradition der eloquentia corporis. ${ }^{131}$ Wenn Jane Goodall das Verhältnis zwischen den Schimpansinnen Flo und Olly beschreibt, dient Individualität zwar zur Auszeichnung der einzelnen Figur, aber dies geschieht im Sinne einer wissenschaftlichen Konzeption vom Sozialleben und -gefüge der Schimpansen, in der die eine (Flo) das dominante, die andere (Olly) das rangniedrige Weibchen ist. Ihre Interaktion, vor allem ihr Konflikt, wird immer auf diese hierarchische Anordnung hin gelesen und die Geste ist dabei Einfallspunkt der theoriegestützten Spekulation. Die Geste wird innerhalb eines theoretischen Rahmens gelesen als Zeichen in einer Aufführung von Sozialverhalten. ${ }^{132}$ Wenn bei Goodall in In the Shadow of Man Flo Ollys Sohn Evered abstraft, eilt Olly herbei, ,uttering threatening barks and looking extremely agitated, but she did not dare join in, and so contented herself when all was over with approaching and, as though to appease the dominant female, laying a hand gently on Flo's back“ (SM, 81 f., Hervorheb. MS). Ebenso wird auch Winkles oben bereits angeführte herbeigeprügelte Unterwerfung und Evereds Antwort darauf in Through a Window als überindividuelle Geste gelesen: „Hastening to crouch before him, with nervous panting grunts, she pressed her mouth to his thigh, kissing him. And then, as is the way of male chimpanzees after aggression, Evered reassured her, grooming her until she relaxed under the gentle caress of his fingers“" (TW, 74, Hervorheb. MS).

Mit Vorliebe bieten die Forschungsmemoiren solch pantomimische Dramen rund um den weiblichen Östrus und sexuelle Begegnungen dar:

After seizing a heap of bananas but before taking a single bite, Goliath stood upright with all his hair on end, stared at Flo [who was flaunting a large sexual swelling], and staggered from foot to foot. As Flo appeared, clutching some bananas herself, Goliath raised one arm in the air and made a sweeping gesture through the air with his banana-filled hand. Flo crouched to the ground, presenting Goliath with her pink posterior, and he mated her in the typical nonchalant manner of the chimpanzee, squatting in an upright position, one fruit-laden hand laid lightly on Flo's back and the other resting on the

\footnotetext{
${ }^{130}$ Brooks verweist in seiner Studie unter dem Begriff ,Aesthetic of Muteness ' sowohl auf die Limitierung des Sprechtheaters im 18. Jahrhundert als auch auf Diderots theaterreformatorische Schriften und Rousseaus Essai sur l'origine des langues. Vgl. Brooks, The Melodramatic Imagination, S. 65 ff.

${ }^{131}$ Zur Theorie und Geschichte der körperlichen Beredsamkeit als Brückenschlag zwischen Anthropologie und Schauspielkunst im 18. Jahrhundert vgl. Košenina, Alexander: Anthropologie und Schauspielkunst. Studien zur ,eloquentia corporis “im 18. Jahrhundert. Tübingen 1995.

${ }^{132}$ Damit erweitert die primatologische Geste den Begriff und die Rolle der Geste, wie sie aus anderen Kontexten bekannt ist: Kappelhoff weist darauf hin, dass die Geste unter der historischen Entwicklung bürgerlicher Individualität einen Bedeutungswandel von der Handlungsbewegung über das Instrument einer gestischen Rhetorik zum „unmittelbaren, körperlichen und stummen Ausdruck[] individueller Empfindungen“ vollzogen habe. Kappelhoff: Matrix der Gefühle, S. 63 f. Košeninas Analyse lenkt den Blick auf die rhetorische Wirkintention der Geste in der Schauspielkunst und deren Wandel im 18. Jahrhundert vom formalisierten Ausdruckund Sprachmittel zum, mit Fokus auf Lessings Empfindungsdramaturgie, Gefühlserzeugungsinstrument. Vgl. Košenina: Anthropologie und Schauspielkunst, S. 31 ff.; 85 ff.
} 
ground beside him. [...] [B] efore Goliath had done with Flo, Fifi was there. Racing up she hurled herself against Goliath, shoving his head with both hands, trying to push him off her mother. I expected Goliath to threaten the child, to hit at her, or at least to brush her aside. Instead he merely turned his head away and appeared to try to ignore Fifi altogether. As Flo moved away, Fifi followed, one hand laid over her mother's swelling, looking back over her shoulder at Goliath, who sat eating his bananas. (SM, 82 f., Hervorheb. MS)

Allein durch die Beschreibung der Handlungs- und Ausdrucksgesten in dieser Passage folgt die Darstellung einem dramatischen Muster: der Anbandelung zwischen Mann und Frau und der Rettung der Mutter durch das Kind, welches den (potenziell gefährlichen) Liebhaber verscheucht. ${ }^{133}$

Hier trifft nun der anthropologische Gehalt im melodramatischen Gestus auf seine Anwendung im Feld seiner Überprüfbarkeit: Wie Brooks anführt, werden der unartikulierte Schrei und die Geste zur Entstehungszeit des (Melo-)Dramas sprachphilosophisch bereits als ,language of nature, the language to which all creatures instinctively have recourse to express their primal reactions and emotions" ${ }^{134}$ konzipiert. Affen scheinen so nicht nur geradezu prädestiniert dazu, Figuren melodramatischer Inszenierung zu sein. An ihnen lässt sich sogar gleichsam die im Melodrama verwirklichte sprachphilosophische These überprüfen, dass der unartikulierte Schrei und die Geste, befreit von aller maskierenden sozialen Konvention, eine ursprüngliche Sprache bilden, die keinen symbolischen, sondern einen emotionalen Gehalt vermittelt. ${ }^{135}$ Als Jane Goodalls ,language far more ancient than words, a language that we shared with our prehistoric ancestors, a language bridging our two worlds“ (SM, 80 f.), ermöglicht diese ursprüngliche Sprache es, primatologische Erkenntnis qua melodramatischem Modus zu gewinnen. Somit werden Affen in einer, Ästhetik der Stummheit" (Brooks) durch die Genre-Irritation in den Forschungsmemoiren dreifach lesbar - als Stellvertreter ihrer Gattung, als Belege sprachphilosophischer Überlegungen und als Illustration affektiver primatischer Gemeinsamkeit, wie sie schließlich in der Pathosformel des berührenden Artkontakts kulminiert (siehe Abschn. 2.2).

Der melodramatische Modus leistet im autobiographischen primatologischen Forschungsbericht also zusammengefasst dreierlei: Erstens macht er schaulustige Beobachtungen sowie Erfahrungen und Erlebnisse von affektiver Herausforderung für die Verschriftlichung in den Forschungsmemoiren vorstell- und interpretierbar. Er liefert eine Perspektive, unter der diverse und ambivalente Ereignisse durch (Genre-)Konventionen zu einer (auch für die Leserschaft) schlüssigen Beobachtungserfahrung geordnet werden können. Zweitens bietet der melodramatische Modus als Darstellungsstil ein moralisches Residuum

\footnotetext{
${ }^{133} \mathrm{Hrdy}$ macht diesen Handlungsakt später zu einer eigenen Sequenz, kategorisiert diese ethologisch als ,sexual harassment" und verweist auf eine (von ihr zurückgewiesene) freudianisch-familiendramatische Deutung des Phänomens durch Harold Gouzoules. Vgl. LA, S. 154 ff.; Gouzoules, Harold: Harassment of sexual behaviour in the stumptail macaque (Macaca arctoides). In: Folia Primatologica 17 (1974), S. 1-19.

${ }^{134}$ Brooks: The Melodramatic Imagination, S. 67 f.

${ }^{135}$ Zur Problematisierung dieser These siehe ebenfalls Brooks: The Melodramatic Imagination, S. $69 \mathrm{ff}$.
} 
an, um Wert- und Affektkonflikte (bspw. jene zwischen der Schaulust der Beobachtungs- als Zuschauer/innen-Position und dem wissenschaftlichen Objektivität- und Neutralitätssauftrag) dank einer erprobten Gefühlsstruktur austragen zu können. Er dient als Instrument, Gefühlsarbeit dialektisch auszuagieren, die aus dem Aufeinandertreffen von individuellem affektiven Orientierungsrahmen, professionellem Verhaltensethos und kulturell bedingten Bewertungsnormen für das mitunter als problematisch wahrgenommene primatische Verhalten erwächst. Der Einsatz eines solchen Modus bietet darüber hinaus eine monopathische affektive Bündelung an, die der Ambivalenz des Beobachteten entgegenwirkt und diese Gefühlsarbeit erleichtern kann. Drittens ermöglicht er durch figurale Zuspitzungen und Stereotypisierungen ebenso wie durch den Einsatz primatologischer Gesten die Lesbarkeit des äffischen Verhaltens sowohl für die Primatologie selbst als auch für die nicht-wissenschaftlichen Rezipient/innen der Forschungsmemoiren. Dass dabei durch die möglichen textuellen Irritationsmomente auch eine Lesbarkeit primatologischer Affektivität entsteht, kann als subversiver Effekt des melodramatischen Modus in den Forschungsmemoiren betrachtet werden.

\title{
3.3 Die Grenzen der Gemeinschaft: Verhaltensforschung zwischen Fallstudie, Kriegsbericht, Trauma und Zeugenschaft
}

\begin{abstract}
This is already a long time ago, I can remember the feelings but I can't still have them. A common prayer for the overattached: You'll let it go sooner or later, why not do it now? Memory print, voices and faces, stories like filament through a piece of time, so attached to the experience that nothing moved and nothing went away. ${ }^{136}$
\end{abstract}

The memory, surely, will always be lurking there, ready to erupt in nightmares in times of sickness, loneliness or depression. (TW, 56)

\subsubsection{Das Problem, Primatische Gewalt ${ }^{\prime}$}

Gewalt ist zugleich „Handlungsoption“ und „,verbunden mit zentralen Momenten der Conditio Humana wie Tod, Macht, Angst, Mut, Verlust, Rausch, Lust, Trauma, Terror, Recht, Unrecht, Freiheit, Zwang“" 137 so Gudehus und Christ in ihrem interdisziplinären Handbuch zur Gewalt. Das macht Gewalt als Gegenstand und Begriff notorisch ,vielfältig und facettenreich“. ${ }^{138}$ Im Rahmen der vorliegenden

\footnotetext{
${ }^{136}$ Herr, Michael: Dispatches. New York 1991, S. 28 f.

${ }^{137}$ Gudehus, Christian/Christ, Michaela: Vorwort und Einleitung. In: Dies. (Hg.): Gewalt. Ein interdisziplinäres Handbuch. Stuttgart/Weimar 2013, S. VII-VIII, hier S. VII.

${ }^{138}$ Gudehus/Christ: Vorwort und Einleitung, S. VII.
} 
Studie interessiert, wie schon an Burroughs' Tarzan-Roman diskutiert, an Gewalt vor allem ihr affektiv ambivalenter Wert: Auf der einen Seite wird Gewalt, vor allem in der ebenfalls ausfransenden Begrifflichkeit des Krieges, in der Politischen Theorie und Philosophie sowie nicht zuletzt in der philosophischen Anthropologie ein epistemischer und identitätsstiftender Gehalt zugesprochen. ${ }^{139}$ Auf der anderen kann gerade der Krieg, aber potenziell auch jeder Akt der Gewalt verstörend, ordnungsauflösend und in der Erfahrung am eigenen Leib traumatisch wirken. In der Anschauung von Gewalt droht, wie u. a. Susan Sontag, Judith Butler und Slavoj Žižek anmerken, im Extremfall eine empathische Überwältigung durch Leid, ${ }^{140}$ zumindest aber affektives ,entrainment ${ }^{6}{ }^{141}$ Nicht zufällig tritt in der Wiederentdeckung des Erhabenen im 20. Jahrhundert Gewalt als Möglichkeit der Erfahrung des Erhabenen in den Diskurs ein. ${ }^{142}$

Dabei wird Gewalt in der Forschung trotz aller Debatten um ihre begriffliche Fassung $^{143}$ fast ausschließlich als menschliches Phänomen definiert, ${ }^{144}$ etwa bei Nunner-Winkler wertneutral als ,absichtvolle physische Verletzung von Menschen

\footnotetext{
${ }^{139}$ Vgl. Münkler, Herfried: Über den Krieg. Stationen der Kriegsgeschichte im Spiegel ihrer theoretischen Reflexion. Weilerswirst 2002, S. $107 \mathrm{ff}$.

${ }^{140}$ Susan Sontags Essay beklagt das Problem der (Kriegs- und Gewalt-)Fotografie, zeitweilig zu bewegen, aber mangels Deutungsangeboten nicht zum politischen Handeln zu aktivieren und so politische Ohnmacht zu produzieren; siehe Sontag, Susan: Regarding the Pain of Others. New York 2003. Judith Butler verweist anhand einer Diskussion von Sontags Thesen auf die Möglichkeit der fotografisch induzierten, zum Handeln aktivierenden Wut; vgl. Butler, Judith: Krieg und Affekt. Hg. u. übers. von Judith Mohrmann/Juliane Rebentisch/Eva von Redecker. Zürich/Berlin 2009, S. 53 ff. Slavoj Žižek hingegen sieht ,something inherently mystifying“ in der direkten Auseinandersetzung mit Gewalt: ,[T]he overpowering horror of violent acts and empathy with the victims inexorably function as a lure which prevents us from thinking“. Žižek, Slavoj: Violence. Six Sideways Reflections. London 2009, S. 3.

${ }^{141}$ Randall Collins versteht unter einem solchen affektiven Einschwingen die menschlich angeborene ,propensity to become caught up in a shared focus or attention and the emotional rhythms of other people“. Collins, Randall: Violence. A Micro-sociological Theory. Princeton/ Oxford 2008, S. 27.

${ }^{142}$ Die Erfahrung des Erhabenen als gewaltvoller Akt einer schockhaften Überwältigung und die Erfahrung von Gewalt als Selbst-transzendierendes ,violent sublime“ in der Folge Edmund Burkes untersuchen u. a. Nieraad, Jürgen: Die Spur der Gewalt. Zur Geschichte des Schrecklichen in der Literatur und ihrer Theorie. Lüneburg 1994, S. 96 und Gomel, Elana: Bloodscripts. Writing the Violent Subject. Columbus 2003, S. xxvii.

${ }^{143}$ Vergleiche zur Vielfältigkeit der Gewaltforschung Heitmeyer, Wilhelm/Soeffner, Hans-Georg (Hg.): Gewalt. Entwicklungen, Strukturen, Analyseprobleme. Frankfurt a. M. 2004.

${ }^{144}$ Eine Ausnahme ist hier z. B. Buschka, Sonja/Gutjahr, Julia/Sebastian, Marcel: Gewalt an Tieren. In: Christian Gudehus/Michaela Christ (Hg.): Gewalt. Ein interdisziplinäres Handbuch. Stuttgart/Weimar: 2013, S. 75-82.
} 
durch Menschen“.145 Spätestens seit Thomas Hobbes' Konzeption des Naturzustands als ,bellum omnium contra omnes “ wird Gewalt gesellschaftsphilosophisch als Kondition des Menschlichen betrachtet. ${ }^{146} \mathrm{Im}$ 20. Jahrhundert wird sie auch ethologisch und phylogenetisch im Menschen verankert und in diesem Sinne nicht nur naturalisiert, ${ }^{147}$ sondern auch normalisiert. ${ }^{148}$ Diese auch der ,Nature vs. Nurture'-Debatte entsprungene Verankerung verdankt sich nicht nur der experimentellen Gewalt- und Aggressionsforschung, die z. B. Hannah Arendt in den 1960ern kritisiert hat, ${ }^{149}$ und die nicht zuletzt durch die Erfahrung der beiden Weltkriege und der Shoah ins Leben gerufen wurde, sondern auch den anschlussfähigen Beobachtungen der Primatologie im Feld.

Die ersten Feldforschenden erleben in ihren Langzeitstudien mehrfach menschliche und tierliche Gewalt, als Opfer, Täter/innen oder Zeug/innen: Jane Goodalls Camp wird in den 1970ern von zairischen Rebellen überfallen. Dian Fossey wird

\footnotetext{
${ }^{145}$ Nunner-Winkler, Gertrud: Überlegungen zum Gewaltbegriff. In: Wilhelm Heitmeyer/HansGeorg Soeffner (Hg.): Gewalt. Entwicklungen, Strukturen, Analyseprobleme. Frankfurt a. M. 2004, S. 21-61, hier S. 28. Nunner-Winkler schließt dabei unter Berufung auf Max Webers Bestimmung von Begriffen als theoretische Konstruktionen, die nicht Ziel, sondern Mittel zum Zweck der Erkenntnis sein dürfen, an eine Definition der Gewaltkommission der deutschen Bundesregierung von 1990 an. Vgl. Weber, Max: Die ,Objektivität' sozialwissenschaftlicher Erkenntnis [1904]. In: Ders.: Soziologie, Weltgeschichtliche Analysen, Politik. Stuttgart 1956, S. 186-262, hier S. 255.

${ }^{146}$ Siehe Hobbes, Thomas: Leviathan or The Matter, Forme and Power of a Common-Wealth Ecclesiasticall and Civil. London 1651.

${ }^{147}$ Vgl. Lorenz, Konrad: Das sogenannte Böse. Zur Naturgeschichte der Aggression. Wien 1965.

${ }^{148}$ Vgl. Meyer, Peter: Grundlagen menschlicher Gewaltbereitschaft. Beiträge evolutionärer Forschung. In: Wilhelm Heitmeyer/Hans-Georg Soeffner (Hg.): Gewalt. Entwicklungen, Strukturen, Analyseprobleme. Frankfurt a. M. 2004, S. 383-410; Euler, Harald A.: Die Beitragsfähigkeit der evolutionären Psychologie zur Erklärung von Gewalt. In: Wilhelm Heitmeyer/Hans-Georg Soeffner (Hg.): Gewalt. Entwicklungen, Strukturen, Analyseprobleme. Frankfurt a. M. 2004, S. 411435. Ein Beispiel für die zugleich naturalisierende und positivistische Gewaltforschung ist Steven Pinkers 2011 vorgelegte Darstellung der gegenwärtigen Epoche als „Neuer Frieden“, siehe Pinker, Steven: Gewalt. Eine neue Geschichte der Menschheit. Frankfurt a. M. 2011 (engl. 2011). $\mathrm{Zu}$ einer fundierten Kritik dieser „Performanz von Modernität durch eine spezifische Kodierung von Gewalt" siehe Martschukat, Jürgen: Gewalt: Kritische Überlegungen zur Historizität ihrer Formen, Funktionen und Legitimierungen. In: Body Politics 1/2 (2013), S. 185-198, hier S. 187.

${ }^{149}$ Arendt stellt an dem ,all-out effort to solve the riddle of ,aggressiveness“ in human behavior" die Übertragung von tierlichem auf menschliches Verhalten epistemologisch und moralisch infrage: ,[T]he research results of both the social and the natural sciences tend to make violent behavior even more of a , natural ${ }^{6}$ reaction than we would have been prepared to grant without them. Aggressiveness, defined as an instinctual drive, is said to play the same functional role in the household of nature as the nutritive and sexual instincts [...]. But unlike these instincts [...] lack of provocation apparently leads to instinct frustration, to ,repressed ' aggressiveness, which according to psychologists causes a damming up of ,energy “ whose eventual explosion will be all the more dangerous. [...] In this interpretation, violence without provocation is ,natural'; if it has lost its rationale, basically its function in self-preservation, it becomes ,irrational', and this is allegedly the reason why men can be more beastly than other animals". Arendt, Hannah: On Violence. San Diego u. a. 1970, S. 59, 62, Hervorheb. im Original.
} 
zu Beginn ihrer Forschungsarbeit im Kongo von Soldaten entführt und - vermutlich - vergewaltigt. Sie liefert sich eine Art bewaffneten Guerilla-Krieg mit den Wilderern in Virunga und wird schließlich ermordet. Biruté Galdikas wird Zeugin und ,Teilnehmerin“ der Vergewaltigung einer Köchin durch einen Orang-Utan. Sie fällt zudem fast einem Tötungsversuch durch ein Orang-Utan-Weibchen zum Opfer. Es ist jedoch die beobachtete intraspezifische Gewalt der Affen, die in der Forschung einen Paradigmenwechsel im Bild unserer nächsten ,Verwandten ‘ einleitet und im Text Spuren der Irritation hinterlässt.

Eine Rolle spielt hier das bereits erwähnte affektive menschliche Verhältnis zum Phänomen der Gewalt: Wie Randall Collins in seiner mikrosoziologischen Analyse und Konflikt-Theorie umreißt, ist Gewalt verbunden mit sowohl einer interaktionalen emotionalen Komponente als auch einer sprachlichen Problematik: In Gewaltsituationen geht es um das ,,intertwining of human emotions of fear, anger, and excitement, in ways that run right against the conventional morality of normal situations“. ${ }^{150}$ Folgt man Collins, so sind Menschen auf ,interactional entrainment and solidarity"151 abgestimmt, sodass konfrontative Situationen ein affektives Gemisch von Anspannung und Furcht (,tension/fear") hervorrufen, das nicht nur die Beteiligten (Kontrahent/innen, potenzielle Täter/innen und potenzielle Opfer) befällt, sondern sich als emotionale Energie auch auf Zuschauer/ innen und Beobachtende überträgt. ${ }^{152}$ Zugleich bietet jedoch der ,ordinary discourse“"153 nicht die Sprache, welche nötig wäre, um Mikrointeraktionen gut zu beschreiben: ,instead it offers a set of clichés and myths that predetermine what people will say“. ${ }^{154}$ Obwohl auch die primatologische Verhaltensforschung Klischees und Mythen verwendet, wie sie im Rückgriff auf den melodramatischen Modus zeigt, ist ihr durch ethologische Schulung eine sprachliche Beschreibung von Gewaltsituationen durchaus möglich. Das affektive Problem stellt sich der Feld-Ethologie eher, wenn sie von bislang ungeahnten gewalttätigen Situationen und Verläufen ,neutral` berichten muss. Hier zeigt sich die große Herausforderung

\footnotetext{
${ }^{150}$ Collins: Violence, S. 4.

${ }^{151}$ Collins: Violence, S. 27. Collins kritisiert die evolutionspsychologische ,Orthodoxie“, mit einem Modell des Menschen als „egotistical gene propagator“ zu operieren (siehe zu diesem Modell auch Abschn. 4.2.1). Dieses Modell gehe von der irrigen Annahme aus, Gewalt sei ,leicht' auszuüben, und vernachlässige dabei empirische Erkenntnisse, denn „humans have evolved to have particularly high sensitivities to the micro-situational signals given off by other humans. Humans are hard-wired to get caught in a mutual focus of intersubjective attention, and to resonate emotions from one body to another in common rhythms. This is an evolved biological propensity; humans get situationally caught up in the momentary nuances of each other's nervous and endocrinological systems in a way that makes them prone to create interaction rituals and thus keep up face-to-face solidarity. [...] We have evolved to be hyper-attuned to each other emotionally, and hence to be especially susceptible to the dynamics of interactional situations" (S. 26 f.).
}

${ }^{152}$ Collins: Violence, S. $19 \mathrm{f}$.

${ }^{153}$ Collins: Violence, S. 4.

${ }^{154}$ Collins: Violence, S. 4. 
der primatischen Gewalt für die menschlichen Beobachter/innen in der Verhaltensforschung.

Im Folgenden soll es um die Beschreibung, Darstellung und Interpretation solcher Gewaltsituationen bei Jane Goodall gehen. Obwohl auch die Funde der anderen Primatolog/innen dazu beitragen, hat die Primatenforschung in den 1970er Jahren nichts so sehr erschüttert wie zwei Phänomene, die Jane Goodalls Forschungspopulationen betrafen: die "cannibalistic attacks of Passion and Pom“ $(T W, 92)$ und der „four-year war“ (TW, 87) der Schimpansen in Gombe. Die „Annihilation of a Community" $(C G, 503)$ und ,the gruesome feasting on the flesh of newborn babies“ (TW, 91) koinzidieren in den Jahren 1974-1977 mit dem Überfall auf das Forschungscamp und der Entführung mehrerer Promovierender durch zairische Rebellen. Diese drei Erfahrungen sind in Goodalls Texten nach 1977 nicht nur untrennbar miteinander verbunden. ${ }^{155}$ Sie sind auch textuelle Wiedergänger. Aufgrund ihrer epistemischen Bedeutung für die Forschung selbst - den Wandel vom ,friedlichen“ Bild der Schimpansen als besserer Version des Menschen (,for so many years I had believed that chimpanzees, while showing uncanny similarities to humans in many ways were, by and large, rather ,nicer" than us"; $T W$, 92) hin zur jenem des menschenähnlich brutalen Mörders (,Suddenly I found that under certain circumstances they could be just as brutal, that they also had a dark side to their nature"; $T W, 92$ ) - tauchen Beobachtungen und Erläuterungen der kannibalistischen Attacken und des Schimpansenkrieges unter Relativierung des Kidnapping-Überfalls mehrfach in Goodalls Werk auf. Sie wirken in den Forschungsmemoiren wie eine epistemische Wunde, die immer wieder aufbricht: „[T]hose events changed for ever my view of chimpanzee nature. [...] And it hurt" (TW, 92). Im Text selbst nimmt die Erfahrung vielfältige Formen an, die wie Narben auf den epistemischen und den emotionalen Schock verweisen.

Im Folgenden soll nun Goodalls textueller Umgang mit diesen beiden erschütternden Beobachtungen unter Schimpansen - dem Kannibalismus und dem ,Krieg ${ }^{6}$ - genauer untersucht werden. Warum sind die Passagen auffällig? Wie verändern sie sich? Was unterscheidet sie? Wie wird in ihnen aus Erfahrungen, Eindrücken und Überlegungen Text gemacht?

Es lassen sich, so die diese Untersuchung bestimmenden Thesen, in Goodalls zweiten Forschungsmemoiren Through a Window zunächst zwei Auffälligkeiten der betreffenden Passagen beobachten. Dies ist erstens die rhetorische Strategie der Darstellung der Geschehnisse, die durch ,fingierte Augenzeugenschaft' eine besondere Eindrücklichkeit der Ereignisse entstehen lässt (Abschn. 3.3.2). Zweitens erfolgt die Narrativierung in je nach Verhaltens-Kategorie verschiedenen Formen (Abschn. 3.3.3). An diesen kann man eine Einordnung der Phänomene auf einem ethologischen Spektrum angemessenen Verhaltens beobachten: hier die Ausnahme des Kannibalismus, die sich der Erklärung entzieht und als Anomalie in der Form des Fallberichts pathologisiert wird (Abschn. 3.3.3.1); dort

${ }^{155} \mathrm{Vgl} . C G$, S. 54 f.; $T W, \mathrm{~S} .91$ und RH, S. 117. 
der schimpansische Auslöschungskrieg, der aufgrund der menschlichen Vertrautheit mit dem Phänomen (und durch den Vergleich mit anderen Forschungsstationen) als speziesgerechtes normales Verhalten im aus dem Kriegsbericht bekannten Detail berichtet wird (Abschn. 3.3.3.2). Das affektive Problem der Gewalt(-Anschauung) lässt sich angesichts solcher (Text-)Wunden und Narben und der Wiederkehr einzelner Details in weiteren Texten Goodalls zudem hinsichtlich einer Trauma-Erfahrung und ihrer Bearbeitung in Form einer Strategie aus der neueren Geschichtsschreibung deuten (Abschn. 3.3.4). Zuletzt soll in den Fokus genommen werden, wie aus der Pluralität von Goodalls Darstellungsweisen von und Reaktionen auf Gewalt in der theoriebildenden Ausdeutung durch Richard Wranghams und Dale Petersons Metastudie Demonic Males eine strategische Homogenisierung primatischer Gewalt vorgenommen wird, die Goodalls Normalisierungstendenz zwar folgt, dem Phänomen intraspezifischer primatischer Gewalt, wie Goodall es auffaltet, m. E. jedoch nicht ganz gerecht wird (Abschn. 3.3.5).

\subsubsection{Wer zeugt für die Affen? (1): Fingierte Augenzeugenschaft als rhetorische Strategie}

Der Anfang einer Erzählung vom Schrecklichen ${ }^{156}$ wird von Goodall deutlich markiert: „Passion amemwua na amemla mtoto wa Gilka“ - Passion has killed and eaten Gilka's infant“" (62, Hervorheb. im Original). Mit dieser Aussage in Swahili beginnt in Through a Window am Ende des Kapitels „Change“ (55 ff.) das Erzählen vom Kannibalismus in Gombe. Die Fallhöhe in dieser Nachricht entsteht durch den vorherigen Verweis auf Goodalls Freude, ,that Gilka had given birth“ (62): „I was delighted“, heißt es hier, „for her first baby had mysteriously disappeared when he was just under a month old.“ Doch dann erreicht sie ,another radio message about Gilka“, und diese ist nicht nur ,distorted and indistinct“, sondern bringt „horrifying news“ (62). Die erschreckende Fremdheit des abjekten Mitgeteilten scheint für Goodall nur in ihrem (für das Publikum), fremden' Wortlaut und der Dopplung durch die Übersetzung adäquat repräsentiert zu sein. Um den epistemischen und emotionalen Gehalt der Nachricht zu verdeutlichen, zitiert Goodall sich

\footnotetext{
${ }^{156}$ Es ist verführerisch, aber nicht unproblematisch, das ,Schreckliche ' hier mit ästhetischen Theorien fassen zu wollen. Denn weder entstammen die Forschungsmemoiren jenem Bereich einer Ästhetisierung von Wahrnehmung, der sich mit Karl-Heinz Bohrer als Literatur untersuchen lässt. Noch sind sie genug der dramatischen Form und ihrem Inhalt verpflichtet, um Nietzsches anthropologischem Tragödienmodell zu unterliegen. Es handelt sich bei den hier rezipierten Phänomenen um den Schrecken lebensweltlicher Gewalterfahrungen, die zwar auch einer „Form der Weltvertextung und Wirklichkeitskonstruktion“ (Nieraad: Die Spur der Gewalt, S. 24) unterliegen, aber nicht jener der literarischen oder künstlerischen Gewaltimagination, die zum Gegenstand der ästhetischen Gewaltforschung geworden ist. Daher soll hier vorerst keine „[n]achnietzscheanische Entgrenzung des ästhetischen Modus“ betrieben werden. Bohrer, Karl Heinz: Die Grenzen des Ästhetischen. Wien 1998, S. 148.
} 
selbst: „,It can't be true. It can't“, I said. And yet I knew it must be. No one could invent such a horrifying incident. ,Oh!' I burst out, , why, why, why did it have to happen to Gilka?"“ (62, Hervorheb. im Original). Was Gilka hier geschieht, ist Folgendes:

Gilka, we were told, was sitting peacefully in the afternoon sun, cradling her tiny infant, when Passion suddenly appeared. She stood for a moment, looking at mother and child then charged towards them, hair bristling. Gilka fled, screaming, but she was doubly handicapped - with an infant to support and a crippled wrist. In a flash she was overtaken. Passion leapt upon her and seized hold of little Otta. Gilka tried desperately to save her baby, but she had no chance and after the briefest of struggles Passion succeeded in snatching Otta away. Then, most macabre of all, she pressed the stolen baby to her breast, and Otta clung there desperately while Passion leapt on Gilka. At this moment Pom, an adolescent at the time, rushed to join her mother, and Gilka, outnumbered, turned and fled with Passion in hot pursuit, Otta still clinging tightly to her belly. Confident in her victory, Passion sat on the ground, pulled the terrified infant from her breast, and bit deeply into the front of the little head: death was instantaneous. Slowly, with utmost caution, Gilka returned. When she was close enough to see the limp and bleeding corpse she gave a single loud, bark-like sound - of horror? despair? - then turned and left.

For the next five hours Passion fed on Gilka's baby, sharing the flesh with her family, Pom and juvenile Prof. Between them they consumed it all, every last scrap. (64 f.)

Ein Jahr später, so schildert Goodall im Anschluss, wiederholt sich das Muster, als Gilka wieder ein Neugeborenes hat:

Five minutes later Passion appeared. Pom at once hurried towards her mother and reached to touch her back, a wide grin of excitement on her face. It was the sort of interaction that occurs between mother and daughter when they get close to a tree laden with delicious fruit. As one, Passion and Pom charged Gilka, who, at first sight of Passion, had begun to flee. Gilka screamed and screamed as she ran, but there were no males nearby to respond to her desperate appeal for help.

Pom raced ahead of Gilka who veered to the side, trying to avoid her. At that moment Passion caught up, seized hold of Gilka and threw her to the ground. Gilka did not try to fight, but crouched protectively over her precious baby. Pom then flung herself into the fray, hitting and stamping on Gilka while Passion seized hold of the infant and bit at its head. Gilka vainly hit at her murderous attacker, while with her free hand she clung desperately to Orion [the infant]. Passion bit Gilka's face and blood poured down from deep lacerations on her brow. Then, working as a team, Passion and Pom together turned Gilka onto her back and, while the stronger Passion grappled with the mother, Pom seized the baby and ran off with him. Then she sat and bit deep into the front of his head. And so Orion was killed in the same brutal way as little Otta the year before. (66)

Gilka ist laut Goodall nicht das einzige Opfer: ,[D]uring the four-year period of their depredations a total of six other newborn infants vanished. I suspect that Passion and Pom were responsible for all these deaths“ (67). Erst als Passion und Pom jeweils selbst trächtig sind, kommt das Muster zu seinem Ende: ,[T]he killings stopped“, „the cannibalistic attacks came to an end and mothers, once again, could travel with their newborn infants without fear" (67).

Da diese Geschehnisse sich zu der Zeit ereignen, in der das Forschungscamp unter Angriff der Rebellen gekommen war, entstammen die in diesen Passagen 
geschilderten Vorfälle der Beobachtung durch einheimische Feldassistenten, die durch den Rückzug der westlichen Forschenden nach Daressalam die alleinige Verantwortung für die Feldforschung übernommen hatten $(C G, 601 \mathrm{ff}$.). Diesen Umstand enthüllt Goodall ihrer Leserschaft in Through a Window auch: Bereits der Einstieg mit dem Swahili-Satz kennzeichnet die Information als Mitteilung, die Goodall medial - durch eine Funknachricht (,radio message“, 64) - erreicht, und die wörtlich übersetzt werden muss. Goodall und ihr zweiter Ehemann Derek reisen auf diese Nachricht hin nach Gombe, wo sie sich die Vorfälle erzählen lassen: „we heard the horrific story in gruesome detail“ (64). Sie werden dabei in die passive Rolle von Zuhörer/innen verwiesen: „Gilka, we were told“ (64, Hervorheb. MS). Die kannibalistischen Vorfälle - ebenso wie später der Schimpansenkrieg - werden in Through a Window jedoch sowohl deutlich als Beobachtung anderer ausgewiesen als auch, wie die obigen Passagen zeigen, als lebendiges Geschehen vor Augen geführt.

Einerseits rückt also der Text durch die Re-Konstruktion der Übermittlung und Erzählung ab von einer Augenzeugenschaft der Autorin und Erzählerin Goodall für das Schreckliche und besitzt so das Potenzial, eher emotional neutralisierende Distanz zum Geschehen anzubieten. Goodall weist sich deutlich als nicht anwesend bei dem ersten beobachteten Vorfall aus. Sie verwendet nicht den Zeugenbericht als ,natürliche Zeugenschaft ${ }^{6}$, die laut Lackey epistemologisch statt des ,speech act of testifying“157 die ,domain of testimony as a source of belief [...], the source whereby hearers acquire information from either the spoken or written word of others"158 liefert. Stattdessen bietet Goodall eine Darstellung, die das Geschehen lebhaft vor Augen führt. So entsteht als Erzählstimme gerade nicht jener Zeuge, der ,die Faktizität des Bezeugten allein und emphatisch durch den Rekurs auf die Authentizität des Wahrgenommenen und Erlebten, dem er beigewohnt hat oder dem er entronnen ist“, ${ }^{159}$ bekräftigt. Dabei wäre es durchaus vorstellbar, die Beobachter/innen dieser Szenen im Text als Zeug/innen auftreten und ein emotionales Zeugnis wirken zu lassen. Goodall verschweigt die potenzielle Zeugenschaft nicht einmal, sondern führt die Feldassistenten Hilali Matama und E. Tsolo als ,observer' an anderer Stelle auf (TW, 48 ff.; CG, 354). An dieser Stelle jedoch lässt sich der Zeugenbericht in Georg Meins oben bereits angeführtem Verständnis formal erst wieder geltend machen, wenn das Ich Goodalls erneut auftaucht. Dass Goodall im nächsten Jahr die zweite Attacke auf Gilka und ihr Neugeborenes selbst beobachtet, wird zweifach durch die Einbindung des „I“ ausgewiesen: „I followed once“, „I stayed with her“ (65). Auch ist es hier Goodall als ,Ermittlerin“ selbst, die die Zuschreibung der anderen kannibalisierten

\footnotetext{
${ }^{157}$ Lackey, Jennifer: The Nature of Testimony. In: Pacific Philosophical Quarterly 87 (2006), S. 177-197, hier S. 178, Hervorheb. im Original.

${ }^{158}$ Lackey: The Nature of Testimony, S. 178, Hervorheb. im Original.

${ }^{159}$ Mein, Georg: Narrative der Zeugenschaft. In: Eva Geulen/Kai Kauffmann/Georg Mein (Hg.): Giorgio Agamben und Hannah Arendt. Parallelen, Perspektiven, Kontroversen. München 2008, S. 223-240, hier S. 226.
} 
Neugeborenen an das gleiche Täterinnen-Gespann mit „I suspect“ (67) vornimmt. Das nächste Mal taucht das „I“ in Zusammenhang mit Goodalls Fürsorge für Gilka im Camp auf. Durch diese Verteilung des beobachtenden Erzählerinnen-Ichs im Text ist Goodall weniger Zeugin der Tatsache, dass Schimpans/innen kannibalisch und mörderisch sein können - was als Bezeugung eines normalen Verhaltens gelesen werden könnte - als Zeugin des außerordentlichen Leidens der einen Schimpansin, Gilka. Die starke Hervorhebung des Ichs und seiner Vertrautheit mit der Schimpansin (,Such was my relationship with her, such was the implicit trust in this human who had kown and loved her since the carefree days of her infancy, that she even allowed me to smear antibiotic cream onto the terrible ulcers on her hands“, 67) entspricht an jener Stelle wiederum ganz dem Gestus der Rede des Zeugen, wie ihn Mein beschreibt als Pathoserzeugung aus der eigenen Position. ${ }^{160}$ Goodall bezeugt dabei das „tale of infinite sadness“ (71), das das außergewöhnlich von ,Schicksalschlägen " betroffene Leben Gilkas geworden ist, ebenso wie ihre verständnisvolle Anteilnahme daran. Die kannibalischen Attacken dagegen sind, wie im Anschluss zu zeigen sein wird, dem Verständnis entzogene pathologische Phänomene. Diese Verteilung ist epistemologisch wie rhetorisch nicht zufällig: Georg Mein weist denn auch darauf hin, dass „Zeugnis und Bericht [...] nicht immer kompatibel [sind], sondern [...] sich auch antagonistisch gegenüber stehen" ${ }^{161}$ können, wenn Konstatieren und Verstehen nicht zur Deckung kommen.

Andererseits bietet gerade der Hinweis auf das Sehen Anderer hier den Auftakt für eine rhetorische Strategie des Vor-Augen-Stellens als Augenzeugenschaft der Leser/innen oder Hörer/innen. Für Sybille Schmidt und Ramon Voges ist dies eine besonders geschickte rhetorische Strategie, weil die Darstellung im Modus der Zeugenschaft „im Pathos der Authentizität ihre eigene kunstvolle Natur als strategische Darstellung verbirgt“. ${ }^{162}$ Denn Goodall installiert sich und Derek hier als Hörende, die durch die Rede anderer das Geschehen erschauen. Das Gehörte wird so in ihrem Bericht der Leserschaft vor Augen geführt und dramatisiert, als sei es gemeinsam erlebt. Mit dieser Verkettung von Zeugenschaft und Hörerschaft als, mit Rüdiger Campe, ,personenbezogene Anschaulichkeit“163 tritt Quintilians enargeia auf: Nach ,[w] [wen we got to Gombe we heard the horrific story in gruesome detail“ und „we were told“ entfaltet sich das Geschehen des

\footnotetext{
${ }^{160}$ „Der Gestus seiner [des Zeugen] Rede gewinnt das Pathos somit aus der eigenen Position. Denn bezeichnenderweise negiert die radikale Isolierung der Subjektposition den Wahrheitswert der Aussage nicht, sondern bekräftigt ihn auf Grund ihrer Einmaligkeit“. Mein: Narrative der Zeugenschaft, S. 226.

${ }^{161}$ Mein: Narrative der Zeugenschaft, S. 228.

${ }^{162}$ Schmidt, Sibylle/Voges, Ramon: Einleitung. In: Sibylle Schmidt/Sybille Krämer/Ramon Voges (Hg.): Politik der Zeugenschaft. Zur Kritik einer Wissenspraxis. Bielefeld 2011, S. 7-20, hier S. 14.

${ }^{163}$ Campe, Rüdiger: Vor Augen Stellen. Über den Rahmen rhetorischer Bildgebung. In: Gerhard Neumann (Hg.): Poststrukturalismus. Herausforderung an die Literaturwissenschaft. Stuttgart/ Weimar 1997, S. 208-225, hier S. 218.
} 
kannibalistischen Überfalls auf Gilka durch Passion (und Pom) im Zusammenwirken von Evidenz und Hypotypose als zeigende Rede. Goodalls Texte leben von der (zunächst weitgehend affektlosen) deskriptiven Qualität der Narration, wie sie Campe in Anschluss an Quintilian als Evidenz fasst, als „kategoriale Transposition des Redens zum Zeigen“. ${ }^{164}$ Im Kapitel „Gilka“ aber ist es die „Figur Hypotypose als Affektmittel schlechthin“ ${ }^{165}$ die „,den Erzähltext einen inne-

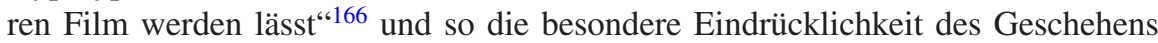
bewirkt. Sie ruft das „Gleichzeitigkeitserlebnis“"167 hervor, mit dem der Redner sich und sein Publikum ,in die Lage des Augenzeugen versetzt“ ${ }^{168}$ Heinrich Lausberg spricht von einer „fingierte[n] Augenzeugenschaft“" ${ }^{169}$ die der/die Redner/ in dem Publikum einrede. Dafür notwendig ist es zunächst, dass der „Redner in seiner eigenen Vorstellungskraft [...] sich zum fiktiven Augenzeugen" macht und dann „das Publikum in diese Augenzeugenschaft hineinzieht“. ${ }^{170}$ Goodalls Verbindung von transparenter Ausstellung der medialen Vermittlung der Nachricht mit ihrer Ankunft in Gombe und der Evozierung einer Erzählsituation schafft in diesem Sinn erst eine fiktive, dann eine fingierte Augenzeugenschaft, mit der sie ihre Leser/innen in das Geschehen zieht: „Gilka, we were told, was sitting peacefully in the afternoon sun, cradling her tiny infant, when Passion suddenly appeared" (64, Hervorheb. MS). Zwar fehlt hier der in der antiken Rhetorik vorgegebene Gebrauch des Präsens. ${ }^{171}$ Doch wird vom Passiv der Hörerschaft für einen Zeugenbericht über die Emphase eines ,course of action“ im Past Progressive Tense bis zur Aktion des Simple Past mit diesem Satz in ein filmisch sich entfaltendes Geschehen geleitet. ${ }^{172}$ Auf das Publikum hat die dann folgende „Detaillierung des Gesamtgegenstandes“ eine potenziell ,,realistische ' $[\ldots]$ und affekterregende [...] Wirkung " ${ }^{173}$ wie sie Lausberg für die fingierte Augenzeugenschaft annimmt.

\footnotetext{
${ }^{164}$ Campe: Vor Augen Stellen, S. 219.

${ }^{165}$ Campe: Vor Augen Stellen, S. 219.

${ }^{166}$ Campe: Vor Augen Stellen, S. 222.

${ }^{167}$ Lausberg, Heinrich: Handbuch der literarischen Rhetorik. Eine Grundlegung der Literaturwissenschaft. München ${ }^{2} 1973$, S. 400.

${ }^{168}$ Lausberg: Handbuch der literarischen Rhetorik, S. 400.

${ }^{169}$ Lausberg: Handbuch der literarischen Rhetorik, S. 401.

${ }^{170}$ Lausberg: Handbuch der literarischen Rhetorik, S. 402.

${ }^{171}$ Vgl. Lausberg: Handbuch der literarischen Rhetorik, S. 402, 404 f. und Campe: Vor Augen Stellen, S. 209.

${ }^{172}$ Albrecht Koschorke bezeichnet in Bezug auf eine solche Dramatisierung des Erzählens, „die das Geschehen wie etwas unmittelbar Erlebtes vor Augen führt“, daher auch als „,szenische Illusion“, die als dramatischer Modus den narrativen des Berichts ablöse. Koschorke: Wahrheit und Erfindung, S. $71 \mathrm{f}$.

${ }^{173}$ Lausberg: Handbuch der literarischen Rhetorik, S. 402.
} 
Ähnlich verfährt Goodall auch in den Passagen, in denen es um den „fouryear war" (TW, 87) geht. Mit dem Begriff bezeichnet Goodall die sich in einem Vierjahres-Rahmen abspielenden Vorfälle, bei denen, nach der graduellen Spaltung ihrer Untersuchungspopulation in Gombe in zwei separate Gemeinschaften - Kasakela und Kahama -, die Mitglieder der Kasakela-Gemeinschaft die Kahama-Gemeinschaft in gezielten tödlichen Überfällen auf einzelne Individuen auslöschen. ${ }^{174}$ Auch hier wird das gewalttätige Geschehen lebhaft vor Augen geführt, auch hier dient die Darstellungsweise der (Augen-)Zeugenschaft als rhetorische Strategie. Dabei wird jedoch die ,natürliche' als epistemische Zeugenschaft stärker zur Unterstützung herangezogen als beim Kannibalismus.

Die Schilderung des ersten Übergriffs der Kasakela-Mitglieder auf ein Kahama-Männchen - ,the first brutal attack“ (88) - wird durch den Hinweis eröffnet, „It was observed by Hilali and one of the other field staff" (88). Dann steigt die Erzählung wieder ein in eine vor Augen gestellte Szene, die die Aufmerksamkeit vollständig bündelt:

The assault began when a Kasakela patrol of six adult males suddenly came upon the young male, Godi, feeding in a tree. So silently had the aggressors approached that Godi was not aware of them until they were almost upon him. And then it was too late. $\mathrm{He}$ leapt down and fled, but Humphrey, Figan and the heavyweight Jomeo were close behind, running shoulder to shoulder, with the others racing after them. Humphrey was the first to grab Godi, seizing one of his legs and throwing him to the ground. Figan, Jomeo, Sherry and Evered pounded and stamped on their victim, while Humphrey pinned him to the ground, sitting on his head and holding his legs with both hands. Godi hat no chance to escape, no chance to defend himself. Rodolf, the oldest of the Kasakela males, hit and bit at the hapless victim whenever he saw an opening and Gigi, who was also present, charged back and forth around the melee. All the chimpanzees were screaming loudly, Godi in terror and pain, the aggressors in a state of enraged frenzy.

After ten minutes Humphrey let go of Godi. The others stopped their attack and left in a noisy, boisterous group. Godi remained motionless for a few moments, lying as his assailants had left him, then slowly got to his feet and, giving weak screams, stood gazing after them. He was badly wounded, with great gashes on his face, one leg and the right side of his chest, and he must have been badly bruised by the tremendous pummelling to which he had been subjected. ( $88 \mathrm{f}$.)

Soweit konstruiert Goodall also wieder eine fingierte Augenzeugenschaft. Doch das ,Schicksal' Godis wird hier mit Hinweis auf das Sehen anderer (oder dessen Ausbleiben) bestimmt: „Undoubtedly he died of his injuries, for he was never seen

\footnotetext{
${ }^{174}$ Goodall benutzt den Begriff , community‘. Im Rahmen der Argumentation übersetze ich mit Hinblick auf den von Helmut Plessner entliehenen Titel des Kapitels unter aller angemessenen Vorsicht der Übertragung von Ideologemen von menschlichen auf nicht-menschliche Tiere ,community“ auch mit ,Gemeinschaft“. Plessners „,blutsmäßige Verbundenheit der Glieder, und darunter [...] sowohl biologische Verwandtschaft als auch geheimnisvollere Gleichgestimmtheit der Seelen“, die „Affektwerte höchsten Grades einschließt“" und eine Rangordnung essenziell bedingt („Den Starken schützt sein Gefolge, es lebt für ihn und aus ihm“), scheint hier zutreffend. Plessner, Helmuth: Grenzen der Gemeinschaft. Eine Kritik des sozialen Radikalismus. Frankfurt a. M. ${ }^{52015}$, S. 43 ff.
} 
again by the field staff and students working in the Kahama community range" (89, Hervorheb. MS). Auf der gleichen Seite wird die Zeugenschaft sogar wörtlich aufgerufen: „Over the next four years, four more assaults of this sort were witnessed" (89, Hervorheb. MS). Im Anschluss an den Überfall auf Goliath, den Goodall als „for me, most tragic of all“ empfindet, wird schließlich ,[o]ne of the students, Emilie“, als Augenzeugin des Vorfalls genannt: Sie „was present during the attack" (89). Nicht nur wird auf ihren emotionalen Zustand verwiesen, um die zuvor geschilderten Vorkommnisse zu bekräftigen: „What shocked her most was the terrifying rage and hostility of the five aggressors" (89). Sie wird sogar wörtlich zitiert: ,,They were definitely trying to kill him.', she told us afterwards. ,Faben even twisted his leg round and round - as though he was trying to dismember an adult colobus after a hunt.““(89). Folgt man Meins Konzeption der aus dem Pathos gespeisten Zeugenschaft, bekräftigt Emilies Aussage nicht nur die Wahrhaftigkeit der Geschehnisse und bietet sich als Garant für die Authentizität der Schilderung und für die zugrunde liegende These eines Vernichtungsschlags an. Ihr bleibt es auch überlassen, das schrecklichste Detail des Angriffs in ,eigene" Worte zu fassen. Die fingierte Augenzeugenschaft der evidenten Schilderung wird somit durch die zitierte Augenzeugenschaft und deren emotionale Aussagekraft ergänzt, um den Eindruck und die Bedeutung der beobachteten Gewalt zu verstärken und zugleich als Lackeys ,testimony as a source of belief' davon zu überzeugen, um was für ein Phänomen es sich hier handelt.

Das Vor-Augen-Stellen scheint bei Goodall als rhetorische Strategie überwiegend der Interaktion von Schimpansen vorbehalten. Augenfällig wird dies, wenn man die Darstellung der kannibalistischen Überfälle und der Kasakela-Angriffe auf die Kahama-Schimpansen mit der Erwähnung des Überfalls auf das Camp vergleicht. Zu Beginn des Kapitels „Change“ wird zwar ,,a sudden night of terror" (55) versprochen, tatsächlich folgt dann jedoch die ,histoire“ als eine sehr knappe Zusammenfassung: „,[F]orty armed men came across the lake from Zaire and kidnapped four of the Gombe students“ (55). Statt den „many confused tales of what had happened“ (55) mit einer genauen Schilderung entgegenzutreten, zählt Goodall schlicht die Fakten auf, unter Hervorhebung der Charakterstärke ihrer einheimischen Mitarbeiter/innen:

My old friend Rashidi was beaten on the head in a vain attempt to make him reveal the whereabouts of the key to the petrol store. He was deaf in one ear for months afterwards. The two young Tanzanian women working at Gombe then, Park Warden Etha Lohay and student Addie Lyaruu, flitted from one student's house to the next, moving quickly throught the dark forest, to warn everyone of the attack. (55)

,[M]oving quickly through the dark forest" dürfte der einzige Ausbruch in Richtung hypotypotischer Darstellungsweise sein. Er wird jedoch gleich eingehegt durch eine andere Form des Erzählens. Womöglich im Versuch, den affektiven Eindruck dieser Zeit als Ganzes abzubilden, schwenkt die Erzählung hier zwischen Perspektiven, Zeiten und Modi hin und her, sodass eher die Verwirrung dieser Episode der Feldforschung als ein genauer, szenischer Ablauf wiedergegeben wird: 
Where had the victims gone? Were they even alive? There were reports of gunshots heard out on the lake, and for days we thought that the hostages might have been killed. It was a time of anguish. Of course we all had to leave Gombe. For a while we stayed at Kigoma, hoping against hope for news of our friends. But none came. A few months before the kidnap I had remarried, and my second husband, Derek Bryceson, had a house in Dar es Salaam. There we all went, $[\ldots]$ and there we waited. Waited, and waited and waited, for what seemed eternity, for news. If it was pure hell for us, those who had not been taken, what of the mental suffering of the victims themselves, and of their parents and other close family? (55)

Goodall schwankt in den mit dem Kidnapping verbundenen Passagen zwischen dem affektiv einschließenden „we“ und ihren persönlichen Gefühlen der Verantwortung: „I shall never forget the relief, the delirious joy, that I experienced on learning that the four were alive and at least physically unharmed" (55). Ganz im Gegensatz zu den anthropomorphisierenden Einsichten in die schimpansische Psyche, die sie gern einsetzt, wird zudem die Unzugänglichkeit der Gefühlswelt anderer Menschen hervorgehoben: „All four eventually recovered from the terrifiying ordeal - at least they seemed to have, judging by their outward appearances" (56).

Obwohl also alle drei Phänomene textuell immer wieder aneinander gekoppelt werden, scheidet die Darstellung der Ereignisse die menschliche deutlich von der schimpansischen Gewalt. Dass diese selbst in der Art der fingierten Augenzeugenschaft noch einmal Differenzen zwischen dem Kannibalismus und dem Vernichtungskrieg zeigt, verweist im Text auf eine eigene Affektlogik der Form.

\subsubsection{Affektlogik der Form}

Dient die rhetorische Strategie der enargeia mittels fingierter Augenzeugenschaft und epistemischem Zeugnis der eindrücklichen Darstellung der Geschehnisse - als hätten auch wir als Leser/innen sie erlebt -, so bietet Goodall in den betreffenden Kapiteln auch Strategien an, diese Geschehnisse ethologisch zu be- und verarbeiten. Als Text bietet Goodalls Through a Window durch verschiedene Genre-Formen eine Einordnung der Vorkommnisse im Spektrum ethologischer Wissensbestände an, die es möglichen machen, sich $z u$ diesen Vorkommnissen angemessen zu verhalten. Mit Helmuth Lethen lässt sich von resultierenden ,Verhaltenslehren' sprechen, die „strategisch angelegte Selbstinszenierungen ein[üben]; ihr Ziel ist das Training eines funktionalen Ich" ${ }^{175} \mathrm{Im}$ Rahmen primatologischer Affektlogiken ist hier das wichtigste Kriterium für die textuelle Selbstinszenierung eines funktionalen Forscher/innen-Ichs angesichts des Neuen die Unterscheidung von ,normalem ' und ,abnormem “ Verhalten. In Through a Window korrespondiert dies, so die These, mit mehr oder minder literarischen Formen wie dem Kriegsbericht auf der einen, der Fallgeschichte und Psychopathologisierung auf der anderen Seite.

\footnotetext{
${ }^{175}$ Lethen, Helmut: Verhaltenslehren der Kälte. Lebensversuche zwischen den Kriegen. Frankfurt a. M. 1994, S. 36.
} 


\subsubsection{Verhaltenslehren des Abnormen: Schimpansischer Kannibalismus als Fallgeschichte und Psychopathologie}

Die primatologischen Forschungsmemoiren verwenden häufig die Struktur von Fallgeschichten, um ihre Daten aufzubereiten. Vor allem der Fokus auf einzelne, aber paradigmatisch eingesetzte weibliche und männliche Individuen und deren ,life stories` zeigt dies. Auch die Sammlung von Verhaltensformen, die an einzelnen Individuen exemplarisch dargestellt werden, gehört dazu. Damit steht die primatologische Feldforschung in der Tradition einer bedeutenden westlichen Wissens- und Erzählpraxis. Susanne Düwell und Nicolas Pethes weisen darauf hin, dass Fälle nicht eine ,mehr oder minder austauschbare Darstellungsweise für ein unabhängig von diesen Darstellungen verhandelbares Wissen“, sondern spätestens ab der zweiten Hälfte des 18. Jahrhunderts in den Humanwissenschaften ,eine zentrale Repräsentations- und Wissensform" ${ }^{176}$ sind. John Forrester geht darüber noch hinaus und sieht den Fall, sein Studium und mit dem 20. Jahrhundert auch terminologisch seine Geschichte - ob als ,Sokratische Methode“ im Recht, als ,Kasuistik $^{6}$ in der Moral ${ }^{177}$ oder in der literarischen Form des Romans und vor allem der Novelle - als ,Denkform sui generis“ ${ }^{178}$ Der Fall zeichnet sich, so fasst Christiane Frey in Anschluss an Forrester zusammen, ,vor allem dadurch aus, dass er das Singuläre, das sich nicht verrechnen lässt, wissenschaftlich relevant macht. " ${ }^{179}$ In diesem Sinne nehmen die primatologischen Forschungsmemoiren eine Repräsentations- und Wissensform und einen Argumentationsstil auf, die beide die ,Wahrnehmung des Menschen als Individuum“ als „weitgehend identisch mit seiner Beobachtung als Fall"180 sehen, wie Marcus Krause mit Bezug auf Michel Foucaults Studien zur Genese der Individualität in der Moderne darlegt. Gerade die Nähe zur und Verwendung der Fallgeschichte in der Literatur und Psychoanalyse macht die Gestaltung des primatologischen Fallberichts interessant. Denn, wie Forrester anführt, im 20. Jahrhundert ist auf (natur-)wissenschaftlicher Ebene

\footnotetext{
${ }^{176}$ Düwell, Susanne/Pethes, Nicolas: Fall, Wissen, Repräsentation - Epistemologie und Darstellungsästhetik von Fallnarrativen in den Wissenschaften vom Menschen. In: Dies. (Hg.): Fall - Fallgeschichte - Fallstudie. Theorie und Geschichte einer Wissensform. Frankfurt a. M./ New York 2014, S. 9-33, hier S. 10. Wie Düwell/Pethes schreiben, ist die veränderte Funktion der Falldarstellungen im Kontext der Anthropologie und der empirischen Humanwissenschaften „die Erforschung neuer Wissensgebiete und der Primat von Beobachtung ohne systematisierende Einordnung" (S. 19). Die primatologische Verhaltensforschung tritt also konsequent nicht nur methodisch, sondern auch formal ihr wissenschaftliches Erbe an.

${ }^{177}$ Vgl. Forrester, John: Wenn p, was dann? In Fällen denken. In: Susanne Düwell/Nicolas Pethes (Hg.): Fall - Fallgeschichte - Fallstudie. Theorie und Geschichte einer Wissensform. Frankfurt a. M./New York 2014, S. 139-168, hier S. 159, 164.

${ }^{178}$ Frey, Christiane: Fallgeschichte. In: Roland Borgards/Harald Neumeyer/Nicolas Pethes/Yvonne Wübben (Hg.): Literatur und Wissen. Ein interdisziplinäres Handbuch. Stuttgart/Weimar 2013, S. 282-287, hier S. 283, Hervorheb. im Original.

${ }^{179}$ Frey: Fallgeschichte.

${ }^{180}$ Krause, Marcus: Zu einer Poetologie literarischer Fallgeschichten. In: Susanne Düwell/Nicolas Pethes (Hg.): Fall - Fallgeschichte - Fallstudie. Theorie und Geschichte einer Wissensform. Frankfurt a. M./New York: Campus 2014, S. 242-273, hier S. 242.
} 
das ,Denken in Fällen“ weitgehend durch einen statistischen Argumentationsstil abgelöst worden. ${ }^{181}$ Die Forschungsmemoiren und ihre Fallgeschichten stellen so auch argumentativ und formal ein Anderes der die Primatologie zunehmend beherrschenden naturwissenschaftlichen Forschungsparadigmen dar.

Die kannibalistischen Angriffe sind in Through a Window in ein Kapitel eingefügt, das nach der Betroffenen der Attacken benannt ist: „Gilka“ (63 ff.). Ähnlich wie in psychoanalytischen Fallgeschichten, geht es in diesem Kapitel nicht allein um die kannibalistischen Vorfälle, sondern zunächst um Gilkas Lebensgeschichte. ${ }^{182}$ Dass diese außergewöhnlich ist, wird bereits mit dem ersten Satz ausgewiesen: „The carefree days of Gilka's life ended when she was about four years old“ (63). Gilkas Leben wird als Kette tragischer Ereignisse geschildert, in der auf jeden kleinen Hoffnungsschimmer nur ein umso tieferer Fall folgt:

How happy for her we were when her infant brother was born. Soon he would be old enough to play with her and her days of loneliness would be over. But then came the grim days of the 1966 polio epidemic when Olly's month-old infant [Gilkas Bruder] became sick and died, and Gilka herself was particularly paralysed in one wrist and hand. Then, as though all this was not enough, two years later Gilka developed a bizarre fungus infection which, by the time she was eleven years old, had hideously disfigured her once elf-like, heart-shaped face. (63, Hervorheb. MS)

Wie sich zeigt, ist Gilkas Lebensgeschichte bereits durch die gesundheitlichen Probleme als medizinische Fallgeschichte ausgewiesen. Zudem vermutet Goodall, wie sie in diesem Kapitel anklingen und in einem anderen Kontext deutlicher werden lässt, ${ }^{183}$ eine Intelligenzminderung (,But Gilka, like Olly before her, was not characterized by great intellectual prowess“, 66), die Gilka besonders anfällig für die Attacken auf ihre Neugeborenen werden lässt. Sie stellt so einen Zusammenhang zwischen diesen Vorfällen und Gilkas Lebensgeschichte her. Ist eine Fallgeschichte mit Krauses Poetologie literarischer Fallgeschichten „eine narrative Darstellung eines Ereignisses im Rahmen einer individuellen Lebensgeschichte, welche in diese Lebensgeschichte in Gestalt einer Krise oder eines Konfliktes eine

\footnotetext{
${ }^{181}$ Forrester verweist hier auf Ian Hackings Untersuchung zur Entstehung des statistischen Denkens. Siehe Forrester: In Fällen denken, S. 140; Hacking, Ian: The Taming of Chance. Cambridge 1990.

${ }^{182}$ Vgl. Bude, Heinz: Freud als Novellist. In: Ulrich Stuhr/Friedrich-Wilhelm Deneke (Hg.): Die Fallgeschichte. Beiträge zu ihrer Bedeutung als Forschungsinstrument. Heidelberg 1993, S. 3-16.

${ }^{183}$ „She [Gilka] is such a sad chimp. That whole history of hers is utterly pathetic. I very much wonder whether the fungus hasn't slightly affected her brain. Because it is so stupid of her to hang around camp“. Goodall, Jane: Dar es Salam Monday [Possibly October 4, 1976]. In: Dies.: Beyond Innocence. An Autobiography in Letters. The Later Years. Hg. von Dale Peterson. Boston/New York 2002, S. 205-207, hier S. 206.
} 
signifikante Zäsur setzt“" ${ }^{184}$ so lässt sich das Kapitel sogar als novellenhaft lesen. Als literarische Fallgeschichte könnte Gilkas Geschichte hier bei Goodall schlicht den Fall eines zwar besonders bemitleidenswerten und geplagten Individuums darbieten, das jedoch exemplarisch für die außergewöhnlichen Vorfälle dieser Epoche der Feldforschung herangezogen wird. Goodall stellt die kannibalistischen Übergriffe in Through a Window jedoch gleichzeitig noch in einen anderen Kontext: jenen der Psychopathologie. Denn während die kannibalistischen Attacken Teil von Gilkas Lebens- als Fallgeschichte sind, sind sie auch die Kulmination der pathologischen Fallgeschichten von Passion und Pom. Und hier wird die Form der Fallgeschichte ambivalent: Zum einen scheint Gilkas Fall exemplarisch zu sein schließlich ist sie, das wird betont, zwar das erzählerisch privilegierte, aber nicht einzige Opfer der Übergriffe. Zum anderen jedoch ist der Kannibalismus eine Anomalie in der schimpansischen Verhaltensnorm, die durch die Pathologisierung der ,Täterinnen ‘ ausgewiesen wird. Die Fallgeschichte wird so von der primatologischen zu einer kriminologischen.

Dass die kannibalistischen Attacken in Gombe außerhalb des ,normalen * schimpansischen Verhaltens liegen, macht Goodall in Through a Window an zwei kurz aufeinander folgenden Stellen im Kapitel „Gilka“ deutlich. Sie unterscheidet erstens diese Attacken von territorialer Gewalt, die auch Kannibalismus beinhaltet. ${ }^{185}$ Der dafür herangezogene Vorfall wird durch eine Reihung von Einwänden - die Kategorisierung des Opfers, die Art und Weise der Gewalt, der Umgang mit der Leiche, sowie der Anteil der Täter am Kannibalismus - als kategorial anderes Phänomen klassifiziert „But that was different for the female was a stranger, an alien, who had aroused the hostility of the males. [...] [H] er infant, it seemed, had been killed almost accidentally. Only a very small portion of the body had been eaten, and only by a couple of the males present" (65, Hervorheb. MS). „By contrast“ (65) zielen Passions und Poms Attacken auf ,the capture of her [Gilka's] baby“ (65) ab, und ,the carcass was consumed in the way that normal prey is consumed, slowly and with relish, each mouthful of meat chewed up with a few green leaves“ (65). Die Kriterien für das spezifische abnorme Verhalten sind die zielgerichtete Erbeutung des Neugeborenen und die Art und Weise des Verspeisens, die Goodall im Rahmen des Jagdverhaltens verortet.

\footnotetext{
${ }^{184}$ Krause: Poetologie literarischer Fallgeschichten, S. 262. Für Krause ist das „Ziel einer solchen Darstellung [...] erstens, einen interpretativen Zusammenhang zwischen Ereignis und Lebensgeschichte herzustellen, in dem einerseits das Ereignis aus biographischen Umständen zumindest teilweise hergeleitet bzw. erklärt werden kann und andererseits das Ereignis generalisierende Aussagen über die Lebensgeschichte erlaubt. Zweites Ziel der Darstellung ist die Herstellung eines Bezugs dieses Ereignis/Lebensgeschichte-Komplexes zu über diesen hinausgehenden Strukturen, Gesetzen, Normen etc. bzw. das Arrangement dieses Komplexes zu einer paradigmatischen Situation, die auf andere Fälle potentiell übertragbar ist" (S. 262 f.).

${ }^{185}$ So merkt sie an: „It was not the first example of cannibalism at Gombe - five years earlier a group of adult males had come upon a female from a neighbouring community, attacked her savagely, and during the fight had seized her baby, killed it, and eaten part of the little body“. $T W$, S. 65, Hervorheb. MS.
} 
Zweitens konstatiert Goodall ein epistemisches Problem: Angesichts eines der sprachlichen Aussage nicht fähigen Personals dieses Kriminalfalls müssen die Motive der beiden Weibchen im Dunkeln bleiben: „Probably we shall never know why Passion and Pom behaved in this gruesome manner" (67). Im Gegensatz zu anderem erstmals beobachteten Verhalten, das Goodall in ihren Texten wiedergibt, unterlässt sie es dabei, sich mit theoriegeleiteten Vermutungen oder empathischen Projektionen ein Verständnis des Neuen anzueignen. Dies ist insofern bemerkenswert, als Goodall im Rahmen ihrer primatologischen Forschungsarbeit stets bemüht scheint, Verhaltensweisen als sinnvoll und evolutionär erklärbar darzustellen. Statt also diese wissenschaftlich indizierten und textuell bereits etablierten Erwartungen zu erfüllen, wendet Goodall ein anderes Verfahren an: In den oben zitierten Passagen selbst wird durch die Wortwahl bereits ausgewiesen, dass das Verhalten und der Gewaltakt in uneindeutiges Terrain fallen. ${ }^{186}$ Halb werden sie in der Art einer militärischen Strategie beschrieben - „,charged towards them“, „, leapt upon her and seized hold“, ,,succeeded in snatching“, ,,rushed to join“, ,,outnumbered, turned and fled“, ,in hot pursuit“, „Confident in her victory“ (alles 64), ,moving silently from the undergrowth", ,working as a team“, ,took the prey“(66) -, halb wird der tödliche Gehalt der Jagd als Mordgeschehen entlarvt: „,her murderous attacker“, ,,attacking her yet again“, „life and death struggle“ (66), „the killings“, „cannibalistic attacks“ (67). Die Einbettung der Passagen in ein Kapitel, das nach der von diesem Gewaltakt betroffenen Schimpansin benannt ist, hebt diese als bedauernswertes Opfer (vor den anderen Betroffenen) hervor. Dies wird dadurch unterstrichen, dass nicht nur wie bereits erwähnt ihre Lebensgeschichte ausgemalt wird, sondern auch die Folgen der Attacken auf Gilka detailliert als Opfer-(Fall-)Geschichte illustriert werden: Zwar hören die Attacken mit den Schwangerschaften der Kannibalinnen auf, doch für Gilka bedeuten sie tatsächlich jene von Krause angemerkte biographische Zäsur, denn ,it was too late. She never really recovered from Passion's murderous attack“ (67). Ihre (körperlichen) Wunden brechen immer wieder auf; „[s]he had been lame before. Now she was truly crippled“ (67). Sie entwickelt ,a chronic diarrhoea which never really left her, and she became increasingly emaciated“ (67). Gilkas Zustand, „her physical condition“, ist so schlecht, dass - im Goodalls Primatologie beherrschenden Reproduktionsnarrativ ein fatales Zeichen - ,[h]er reproductive days were over“ (67). Goodall überträgt die Wirkung

\footnotetext{
${ }^{186}$ Inwiefern der Kannibalismus selbst ein heterogenes Objekt der Faszination ist, zeigen in Bezug auf die Anthropophagie u. a. Foucault, Michel: Die Anormalen. Vorlesungen am Collège de France (1974-1975). Frankfurt a. M. 2003 (frz. 1999), S. 133-177; Košenina, Alexander: Kasualpoetik des Wilden. Fälle von Kannibalismus in Thüringen (1771) und Neuseeland (1773). In: Jörg Robert/Friederike F. Günther (Hg.): Poetik des Wilden. Festschrift für Wolfgang Riedel. Würzburg 2012, S. 247-259; Düwell, Susanne: Populäre Falldarstellungen in Zeitschriften der Spätaufklärung: Der spektakuläre Fall des ,Menschenfressers' Goldschmidt. In: Dies./Nicolas Pethes (Hg.): Fall - Fallgeschichte - Fallstudie. Theorie und Geschichte einer Wissensform. Frankfurt a. M./New York 2014, S. 293-314; sowie zum Kannibalismus als „Ermöglichungsfigur" Hein, Claudia: Die Essbarkeit der Welt. Italo Calvino, Marianne Wiggins, Juan José Saer. Bielefeld 2016, S. 53 ff.
} 
der krisenhaft eingebundenen Attacken auch auf einen seelischen Zustand: „She had been lonely before but she was infinitely more so now" (67). Geselligkeit sucht Gilka nur noch bei anderen Außenseiterinnen, ,the big, sterile Gigi and the immigrant Patti“ (67), die sie jedoch auch bald verlassen: ,When her friends set of for new pastures Gilka was left by herself. [...] She would sit, a small lonely figure, gazing out over the valley, watching and waiting“ (67). Nach einer „long series of misfortunes“ stirbt Gilka als tragische Protagonistin: ${ }^{187}$ „Her life, begun with such promise, had unfolded into a tale of infinite sadness“" (67).

Das abnorme Verhalten der „Passion family“ (67) wird allerdings nicht nur durch die Opferpathologie Gilkas hervorgekehrt. Goodall verfolgt eine Langzeit-Textstrategie, um die beiden Schimpansinnen als Anormale ${ }^{188}$ darzustellen und zugleich die epistemische Lücke zu füllen, die die Frage nach den Motiven für den Kannibalismus aufgetan hat. Dieses Verfahren beginnt bereits einige Kapitel zuvor, unter dem Titel „Mothers and Daughters“(27) mit einer systematischen Zeichnung von Passion und Pom als problematisches Mutter-Tochter-Gespann und verhaltensauffällige Individuen. So ist „Pom’s childhood [...] bleak“, denn:

\begin{abstract}
Passion's personality was as different from Flo's as chalk from cheese. Even when I knew her in the early sixties she was a loner. She had no close female companions, and on those occasions when she was in a group with adult males her relationship with them was typically uneasy and tense. (28)
\end{abstract}

Passion ist eine „cold mother, intolerant and brusque“ (28), die kaum mit ihrem Kind spielt. Pom ist der zeitgenössischen Entwicklungspsychologie folgend ${ }^{189}$ daher ein ,anxious and clinging child“; es ist die Rede von „Pom's depression“ (28). Goodalls Wortwahl bedient sich deutlich bei psychologischer Terminologie, auch wenn es weiter heißt: ,[T]hese signs of improved psychological wellbeing disappeared during the trauma of weaning“ (28). Durch Poms ,inability to cope“ (28) kehrt ihr ,,sense of insecurity“ (29) zurück und und sie ,reverted to the

\footnotetext{
${ }^{187}$ Gilka lässt sich vor allem im Verständnis der jüngeren Aristoteles-Rezeption, wie sie Terry Eagleton wiedergibt, als tragisch lesen. Denn das Tragische ist ,the recognition of a strain of insouciant refractoriness to human agency that is woven into the very fabric of action itself, a recognition of the inability of agents to guarantee their well-being and happiness even when they attempt, correctly, to found that well-being and happiness on the cultivation of moral virtue and deliberation“, wie Eagleton Aryeh Kosman zitiert. Eagleton, Terry: Sweet Violence. The Idea of the Tragic. Malden u. a. 2003, S. 78, Hervorheb. im Original; Kosman, Aryeh: Acting: Drama as the Mimesis of Praxis. In: Amélie Oksenberg Rorty (Hg.): Essays on Aristotle's Poetics. Princeton 1992, S. 51-72, hier S. 66.

${ }^{188}$ Foucault versteht die Anormalen als Individuen, ,die zwar als Kriminelle verurteilt, jedoch in Begriffen des Normalen und Pathologischen beurteilt und kategorisiert werden“. Foucault: Die Anormalen, S. 123.

${ }^{189}$ Goodalls Vokabular verweist auf die ,Refrigerator Mother Theory “ der 1950er Jahre, siehe Leo Kanners Studien des kindlichen Autismus und Bruno Bettelheims psychoanalytische Konzeption der ,kalten Mutter'. Kanner, Leo: Autistic disturbances of affective contact. In: Acta Paedopsychiatrica 35/4 (1968), S. 100-136; Bettelheim, Bruno: The Empty Fortress. Infantile Autism and The Birth of The Self. New York 1967.
} 
depressed state in which she had been“ (30). Pom bleibt „lethargic and listless“ (29), „developed strange, neurotic behaviours“ (30) und ,in a vicious circle, her nervousness and tension increased" (30). Besonders das Verhältnis zwischen Mutter und Tochter wird als auffällig beschrieben, als „unusually close relationship“ (32). Diese unnatürliche Nähe zwischen Mutter und Tochter wird durch Beispiele hervorgehoben, in denen Passion entweder bei der Anbandelung von Sexualakten durch Männchen interveniert oder ihre Tochter bei deren ,consortships ‘ begleitet. Im selben Kapitel wird erzählerisch vorausgegriffen und auch die Zeit nach den kannibalistischen Attacken pathologisiert: Mit ihrem eigenen Nachwuchs weiß Pom besser umzugehen, als Goodall ihr zugetraut hat, aber: ,there was something lacking“ (33). Wenig überraschend stirbt Poms Baby, weil sie es nicht (richtig) beaufsichtigt. Goodalls Unwohlsein an der Figur Pom zeigt sich in einem Vergleich mit der so vorbildhaften wie für schimpansisches Verhalten prototypischen Flo-Familie, der durch die Praeteritio auffällig ist: „It would be unfair to blame Pom entirely for the accident, to accuse her of negligence. [...] Yet I cannot imagine [Flo's daughter] Fifi losing a child in this way" (34, Hervorheb. MS).

Goodall konstruiert in Through a Window somit rund um die kannibalistischen Attacken der Passion-Familie Fallgeschichten von Opfern und Täterinnen. Gerade durch die Pathologisierung des Verhaltens in der Mutter-Tochter-Beziehung wird versucht, die Gründe des kannibalischen Verhaltens, „,[d]as große Außerhalb, die große Andersheit, wie sie seit dem 18. Jahrhundert durch unsere rechtlich-politische Innenwelt festgelegt ist", ${ }^{190}$ als tendenziell unergründliche psychopathische Akte oder als psychogenetisch angelegtes abnormes Verhalten festzuschreiben.

\subsubsection{Verhaltenslehren des Normalen: Annihilation einer Gemeinschaft als Kriegsbericht}

Die Auslöschung der Kahama-Gemeinschaft durch die Kasakela-Gemeinschaft wird dagegen bereits mit dem Titel des betreffenden Kapitels in Through a Window als ein durchaus bekanntes, ,normales“ Phänomen kategorisiert: „War“ (83). Das Kapitel beginnt mit einem szenischen Einstieg, dessen Sprachgebrauch und Anbindung die Vorkommnisse als kriegerisches Verhalten einordnen. Nicht nur ist ,attack“ die häufigste Vokabel, die „Kasakela patrole [...] penetrated ever deeper into the territory of the Mitumba Community“ (83, Hervorheb. MS). Das Männchen Satan ist ,in the lead“ (83), andere „hurled themselves into the fray“ (84). Die ,,aggressors“" (88) rennen „shoulder to shoulder“ (88). Es gibt in den ,,assaults“ (89) ,,seizing“ (88) von Körperteilen und die Hoffnung, dass einzelne Individuen ,„,defected “ to enemy ranks“ (90), dass sie ,could somehow gain admittance to the Kalande ranks“ (91). Die Opfer der Übergriffe sind schließlich wie Kriegsverletzte „,badly wounded“ (89). Zwar zeigt Goodall im Verlauf der Auslöschung der südlichen Kahama-Gemeinschaft zunehmend ein Befremden am Ausmaß der Gewalt, welches in die Darstellung einfließt: Attacken werden „,brutal“ (88) und ,in a vicious way“ (90)

${ }^{190}$ Foucault: Die Anormalen, S. 141. 
ausgeführt, die Opfer „brutally murdered“ (91). Doch die Vorkommnisse werden bereits früh in Zusammenhang mit ,border patrols“ gesetzt. Durch adverbiale oder phrasale ,Marker' wird in dieser Anbindung das Verhalten als ,normal' identifiziert:

At least once a week the Gombe males, usually in groups of not less than three, visit the peripheral areas of their community range. [...] When the males discover some good source of food [...], they often go back [...]. On expeditions of this sort, the chimpanzees typically ascertain the whereabouts of their neighbours [...]. (84, Hervorheb. MS)

Durch das ethologische Präsens, welches sich hier vom erzählenden Imperfekt der Darstellung des gewalttätigen Geschehens abhebt, wird diese Art von Verhalten als für Schimpansen nicht pathologische Form präsentiert. So kann das Phänomen als Teil territorialen Verhaltens gelesen und in der Auflistung der Verhaltensformen in Bezug auf die unterschiedlichen Möglichkeiten der konfliktiven Begegnung an ,primitive' menschliche Kriegsführung, z. B. Grenzkonflikte, angebunden werden. Besonders auffällig ist dies, wenn die Akteur/innen nicht mehr als Schimpansen ausgewiesen werden und die Schilderung Szenen der ritualisierten Kriegsführung aus Robert Garners ethnologischem Dokumentarfilm Dead Birds (1964) aufruft:

\begin{abstract}
If honours are about equal - with similar numbers of males in each patrolling group - the members of both sides, usually keeping several hundred yards apart, hurl threats at each other. First one group, and then the other, performs wild displays, charging through the undergrowth, $[\ldots]$ and all the while uttering loud, fierce calls. Finally, after half an hour or more, each side retreats towards the safe central part of its own home range. This vigorous and noisy behaviour serves to proclaim the presence of the legitimate territory owners and to intimidate the neighbours. Fighting is not necessary. $(85)^{191}$
\end{abstract}

Dass die Vorkommnisse als Krieg gelesen werden können, verdankt sich jedoch vor allem der Darstellung im Modus des Kriegsberichts. ${ }^{192}$ Es wurde bereits darauf hingewiesen, dass der Kriegsbericht eine ethnografische Dimension hat

\footnotetext{
${ }^{191}$ In Gardners auf der Harvard-Peabody-Expedition ins westliche Hochland Neuguineas entstandenen Filmaufnahmen, die die Dani-Ethnie in kriegerischer Auseinandersetzung präsentieren, sieht Randall Collins die Illustration eines Musters, in dem „fighters are mostly fearful and incompetent in their exercise of violence [...] when they are evenly matched". Collins Beschreibung der Szenen aus Gardners Film sind verblüffend nah an Goodalls Beschreibung der Schimpansen: „The fighters consist of all the adult males of two neighboring tribes [...]. They meet at their traditional fighting ground on the border between their territories. The film shows a dozen or so fighters in the forefront, with an individual or two darting forward to shoot an arrow at the enemy side; when this happens, the other side falls back. The battle has a rhythmic pattern of waves, rushing forward and then back, as if there was a magnetic force that keeps even the bravest fighters from going far beyond the dividing line“. Collins: Violence, S. 40; Gardner, Robert: Dead Birds. Documentary Educational Resources 1964.

${ }^{192}$ Der Begriff ,Kriegsbericht' soll hier wörtlich als ,Bericht von einem Krieg‘ gefasst werden. Die Vielfalt der Berichterstattung und Narrativierung von Krieg macht es schwierig, neben dem Thema genrespezifische Merkmale zu isolieren. Vgl. die Vielfalt von Texten, die zur Studie von Kriegsdarstellungen herangezogen werden können, z. B. bei Norris, Margot: Writing War in the Twentieth Century. Charlottesville/London 2000, S. 5 ff.
} 
und ein Extremfall teilnehmender Beobachtung sein kann. ${ }^{193}$ Ein Extremfall ist der Krieg aufgrund seiner radikal affizierenden Wirkung, denn „,[d]ie Gefährdung des Körpers (Tod und Zerstückelung) erlaubt es nicht, eine sichere Beobachterposition einzunehmen. Im Krieg ist für den teilnehmenden Beobachter die Teilnahme bis zum Äußersten gefährdert", ${ }^{194}$ so Daniela Kirschstein. Dabei fungiert der Krieg von Menschen für Menschen in der Interpretation als „Ausdruck und Erfahrung radikaler Fremdheit", ${ }^{195}$ der der Kriegsbericht bzw. die Kriegsliteratur als „Reaktion auf eine radikale Situation der nicht mehr in bekannten Deutungsmuster zu bringenden Erfahrung der modernen kriegerischen Gewaltszenarios und zugleich als Konstruktion einer solchen Situation"196 entgegentritt. Dies gilt gerade dort, wo die Ethnographie angesichts des Kriegs als radikaler Fremdheitserfahrung in der Mitte der eigenen Kultur als Deutungsinstrument ,versagt ‘ ${ }^{197}$

Der Krieg wird jedoch seit Hegels Phänomenologie des Geistes nicht nur als Zusammenbruch zivilisatorischer Ordnung und Einbruch des Unmenschlichen, sondern paradoxerweise auch als krisenhaftes Medium der Hervorbringung des Menschen konzipiert, wie Margot Norris mit Hilfe von Alexandre Kojèves Hegel-Lektüre hervorhebt: ,a theory not that war is the result of humankind's unsuppressed animality or bestiality but, on the contrary, that war is coeval with the moment of becoming human“. 198 Für Hegel bewährt sich das menschliche Selbstbewusstsein durch den Kampf auf Leben und Tod: „Das Individuum, welches das Leben nicht gewagt hat, kann wohl als Person anerkannt werden; aber

\footnotetext{
${ }^{193}$ Vgl. Lubrich, Oliver: Das Schwinden der Differenz. Postkoloniale Poetiken. Bielefeld 2009, S. 151.

${ }^{194}$ Kirschstein, Daniela: Writing War. Kriegsliteratur als Ethnographie bei Ernst Jünger, Louis-Ferdinand Céline und Curzio Malaparte. Würzburg 2014, S. 10. Wie eine solche (affektive) Gefährdung selbst die ausgewiesenen Nicht-Kombattant/innen unter den Berichterstatter/ innen betrifft, schildert eindrücklich Michael Herr in seinem Vietnamkriegsbericht: „Talk of impersonating an identity, about locking into a role, about irony: I went to cover the war and the war covered me; an old story, unless of course you've never heard it. I went there behind the crude but serious belief that you had to be able to look at anything, serious because I acted on it and went, crude because I didn't know, it took the war to teach it, that you were as responsible for everything you saw as you were for everything you did. The problem was that you didn't always know what you were seeing until later, maybe years later, that a lot of it never made it in at all, it just stayed stored there in your eyes. Time and information, rock and roll, life itself, the information isn't frozen, you are“. Herr: Dispatches, S. 19 f.
}

${ }^{195}$ Kirschstein: Writing War, S. 13.

${ }^{196}$ Kirschstein: Writing War, S. $11 \mathrm{f}$.

${ }^{197}$ Der Kriegsbericht ist jedoch nicht nur Ethnographie - oder lässt sich als eine solche lesen, dort wo die Ethnologie die Waffen streckt -, sondern letztlich auch Ethographie, indem er das Verhalten im Krieg beobachtet, beschreibt und zu deuten versucht. Erst wenn sich das Verhalten nicht mehr erklären lässt, das zeigt z. B. wiederum Herrs Dispatches, tritt der Ordnungsbruch ans Tageslicht.

${ }^{198}$ Norris: Writing War, S. 16. „The transition from animal to human required the willingness to risk life, to transcend the survival instinct and set immaterial values above material life", so fasst Norris die ,fiction $[\ldots]$ of war's genesis“ zusammen. 
es hat die Wahrheit dieses Anerkanntseins als eines selbstständigen Selbstbewußtseins nicht erreicht."199 Das eigene Leben zu riskieren, heißt in der Dialektik von Herr und Knecht aber auch und wesentlich, den Tod des Anderen anzustreben:

\begin{abstract}
Ebenso muß jedes auf den Tod des Anderen gehen, wie es sein Leben daransetzt; denn das Andere gilt ihm nicht mehr als es selbst; sein Wesen stellt sich ihm als ein Anderes dar, es ist außer sich, es muß sein Außersichsein aufheben; das Andere ist mannigfaltig befangenes und seiendes Bewußtsein; es muß sein Anderssein als reines Fürsichsein oder als absolute Negation anschauen. ${ }^{200}$
\end{abstract}

Kojèves Hegel-Kommentar hebt stärker noch die Differenzierung des Menschen vom Tier durch die Überwindung ,seine[r] tierische[n] Begierde“ also der „Begierde nach Lebenserhaltung“201 hervor und setzt als ,,jede menschliche, anthropogene, das Selbstbewußtsein, die menschliche Wirklichkeit produzierende Begierde [...] letztlich eine Funktion der Begierde nach Anerkennung an“. ${ }^{202}$ Der Kampf um Anerkennung ist der ,Ursprung' menschlichen Selbstbewusstseins. Dieses menschliche Wesen braucht ein ebenbürtiges Anderes, um sich in einem Kampf auf Leben und Tod zu konstituieren, begegnet jedoch dem Problem, dass einerseits der Tod des Anderen entscheidend für seine Wesenheit ist, andererseits jedoch der Tote keine Anerkennung mehr zollen kann, so also keine Verwirklichung der Menschlichkeit möglich ist. Wie Kojève zusammenfasst:

Wenn sich die menschliche Wirklichkeit als ,anerkannte "Wirklichkeit konstituieren soll, müssen die beiden Gegner nach dem Kampfe am Leben bleiben. Das ist aber nur möglich, wenn sie sich im Kampfe verschieden verhalten. [...] Der eine muß [...] vor dem anderen Angst haben, ihm gegenüber nachgeben und den Einsatz seines Lebens zum Zwecke der Befriedigung seiner Begierde nach Anerkennung ablehnen. Er muß seine Begierde aufgeben und die des anderen befriedigen: er muß ihn anerkennen, ohne von ihm anerkannt zu werden. ${ }^{203}$

Aus dieser Genese von Sieger und Unterworfenem, Herr und Knecht, wird bei Hegel die Grunddisposition der Menschheit hergeleitet.

Wenn Goodall die Vorfälle in Gombe mit dem Begriff ,Krieg ' belegt, verweist sie nicht auf eine Fremdheit, sondern auf etwas Bekanntes, nicht auf die Alterität, sondern auf die Ähnlichkeit von Mensch und Schimpanse. Zugleich scheint sie Hegels Anthropogenese aus der gewalttätigen Auseinandersetzung zu kommentieren, indem sie das ,neue“ Verhalten der Schimpansen als ,precursor of

\footnotetext{
${ }^{199}$ Hegel, Georg Wilhelm Friedrich: Phänomenologie des Geistes. Frankfurt am Main: Suhrkamp 1986 (G.W.F. Hegel Werke, Bd. 3., Hg. von Eva Moldenhauer und Karl Markus Michel), S. 149. Hervorheb. im Original.

${ }^{200}$ Hegel: Phänomenologie des Geistes, S. 149.

${ }^{201}$ Kojève, Alexandre: Hegel. Eine Vergegenwärtigung seines Denkens. Ein Kommentar zur Phänomenologie des Geistes. Hg. von Iring Fetscher. Frankfurt a. M. 1996 (frz. 1947), S. 24.

${ }^{202}$ Kojève: Hegel, S, 24.

${ }^{203}$ Kojève: Hegel, S. 26.
} 
warfare“ ( $C G, 530)$ deutet und als gewalttätige Form beschreibt, die nicht den idealistischen „Prestigekampf auf Leben und Tod“204 zweier ebenbürtiger Wesen darbietet, sondern jene von Collins identifizierte Hauptstrategie illustriert, die (menschliche) emotionale Gewalthemmung (,confrontational tension and fear“205) zu überwinden: „[F]ind a weak victim to attack“. ${ }^{206}$ In Goodalls Passagen des ,four-year war' attackiert eine Gruppe ein einzelnes Individuum oder eine Mutter-Kind-Gruppe. ${ }^{207}$ Theoretisch können diese Opfer die Übermacht - das Selbstbewusstsein - der Angreifer anerkennen, da sie noch lebend aus der gewalttätigen Begegnung herauskommen. Tatsächlich zeigt Goodall diese Opfer häufig in einem symbolischen letzten Bild, wie sie ihren Angreifern hinterherblicken:

Godi remained motionless for a few moments [...], then slowly got to his feet and, giving week screams, stood gazing after them. $(T W, 88 \mathrm{f}$.)

Goliath [...] managed to sit, but with difficulty, and as he gazed after his one-time companions he was trembling violently. $(T W, 89)$

In Goodalls Vermutungen erliegt das Opfer jedoch stets im Anschluss seinen Verletzungen.

Das Genre des Kriegsberichts bewirkt für die Primatologie also vorwiegend die Einordnung der verstörendsten Beobachtungen schimpansischer Gewalt in vertraute Deutungsmuster, die dem neuen Verhalten seine Fremdheit nehmen. Dies gelingt in Through a Window durch ästhetische Mittel, die nicht unbedingt Genre-spezifisch sind, aber gerade im thematischen Zusammenhang mit dem Krieg für beruhigende Ordnung im Text sorgen. Denn sie wirken immersiv: das Evozieren atmosphärischer Bilder, die Figurenzeichnung - das Identifizieren der ,Kombattant/innen“ und die Ausführung ihrer Einzelhandlungen - und die Identifizierung emotionaler Zustände, letztlich das Narrativ eines strategisch geführten Krieges. Um die Atmosphäre des ersten Vorfalls zu etablieren, heißt es bei Goodall: „It was a wind-still day and the forest was silent but for the periodic shrilling chorus of the cicadas. Suddenly a twig snapped, a sharp, brittle sound“ (83). Die Mitglieder der Patrouille, Satan, Gigi, Figan, Evered, Mustard und Goblin, werden im ersten Absatz alle einzeln benannt und differenziert. Die Schimpansen dieser Gruppe lassen sich in der Szene durch individuelle Figurenzeichnung unterscheiden. Satans Grinsen, „his face split by a wide grin“, ist ,, gash of white teeth set in bright pink gums“ (83). Gigi, ,fully pink“, ist beim Angriff auf das unbekannte Weibchen ,getting in a hit whenever opportunity offered“ (84), „obviously determined to have the last word“ (84). Goblin, wagemutiger als die

\footnotetext{
${ }^{204}$ Kojève: Hegel, S. 25.

${ }^{205}$ Collins: Violence, S. 9.

${ }^{206}$ Collins: Violence, S. 9.

207 ,It is when two or more males encounter a lone stranger or a couple of stranger females with infants, that fierce and brutal attacks take place“ (TW, S. 85). Die Angriffslust wird im einführenden Beispiel sogar durch den Ruf eines Schimpansen-Kindes ausgelöst, vgl. TW, S. 83.
} 
anderen, ist, nachdem das Opfer flieht, ,the only male who followed“ (84). Die Schimpansen unterscheiden sich auch in ihrer Angriffslust, mancher ,roaring his defiance, still slapping and stamping on the ground and drumming on the tree trunks“ (87), andere, „chicken-hearted companions“(87), „turned and fled in silence“ (87). Die ,Täter/innen“ bzw. ,Angreifer/innen“ hier stehen in engem und bestätigendem körperlichem Kontakt miteinander, sie sind Figuren einer eng verbundenen Gruppe, „their arms around one another" (83): „Satan turned to the others [...]. Silently he embraced Jomeo who was behind him. Figan and Evered also threw their arms around one another. Mustard reached to touch Goblin. Like Satan, all were grinning hugely“ (83). Der Text stellt über Körperlichkeit eine Gemeinschaft her, die durch extreme körperliche Gewalt ihre Grenzen zieht.

Goodalls Zuschreibungen lassen sich mit Randall Collins mikrosoziologischen Beobachtungen zu Gewalt und ihrem emotionalen Fundament decodieren: Satans Grinsen ist Ausdruck von ,,part fear, part excitement“ (83). Die Kasakela-Schimpansen sind in ,a state of excitement that bordered on frenzy“ (88), ,in a state of enraged frenzy“ (88), nach dem Angriff dann in ,,a noisy and boisterous mood“ (84), „[a]nd all the time they called out, as though in triumph“ (89). Goodall geht soweit, in , , $[\mathrm{t}]$ his sudden sense of purpose, this air of determination“ (84 $\mathrm{f}$.), ein ideologisches Motiv für die Angriffe auszumachen: „It seems, then, that the attacks are an expression of the hatred that is roused in the chimpanzees of one community by the sight of a member of another" (86). ${ }^{208}$

Im Kontext der anderen Vorkommnisse dieser signifikanten Jahre 1974-1977 in Gombe wird zunächst das Novum des Verhaltens hervorgehoben: ,a different kind of violence altogether" (92). Doch anders als beim Kannibalismus arbeiten diese Passagen ein Verständnis des neuen Verhaltens heraus. Sie bedienen sich weniger einer rhetorischen Strategie, die schockierend konstatiert statt versteht, als einer Art des ,Reenactments ${ }^{609}$ als Form eines szenisch nachformenden Verstehens.

\footnotetext{
${ }^{208}$ Goodall verwendet hier einen Baustein für eine Theorie evolutionär wurzelnden genozidalen Verhaltens wieder, den sie bereits in The Chimpanzees of Gombe gesetzt hat. Dort stellt sie aufgrund ihrer Beobachtungen der Konflikte zwischen den Schimpansen-Gemeinschaften die Vermutung auf, dass ,genocide [...] played a major role in group selection“ (CG, S. 530). Sie stellt für die Vorfälle in Gombe fest: „The victims are treated more as though they were prey animals; they are ,dechimpized““ (S. 532). Schließlich wagt sie einen Ausblick auf das Potenzial dieser großen Ähnlichkeit von Menschen und Schimpansen in der Gewalt, die wieder an Hegels Anthropogenese erinnert: „The chimpanzee, as a result of a unique combination of strong affiliative bonds between adult males on the one hand and an unusually hostile and violently aggressive attitude toward nongroup individuals on the other, has clearly reached a stage where he stands at the very threshold of human achievement in destruction, cruelty, and planned intergroup conflict. If ever he develops the power of language - and, as we have seen, he stands close to that threshold, too - might he not push open the door and wage ware with the best of us?" (S. 534).

${ }^{209}$ Zum (historiographischen) Verstehen durch die mentale Rekonstruktion qua Reenactment, ,[to] follow a present performance [...] actively, in that sense performing it again in imagination“, vgl. Dray, William H.: History as Re-enactment: R. G. Collingwood's Idea of History. Oxford 1995, S. 33.
} 
Goodall vollzieht eine Einordnung der schimpansischen Gewalt in die Deutungsmuster eines bekannten Phänomens. Durch das Ableiten von Theorien zum territorialen Verhalten von Schimpansen und die Analogisierung zum menschlichen Krieg (und gar zum Genozid) scheint hier aus dem neuen Verhalten erstens Normalität, zweitens eine bedeutsame charakteristische Verwandtschaft zum Menschen und drittens eine evolutionäre Verankerung menschlicher als primatischer Gewalt gewonnen zu werden.

\subsubsection{Wer zeugt für die Affen? (2): Epistemologisches Trauma und,empathic unsettlement'}

Als Verhaltenslehren dienen die verwendeten Formen in den Kapiteln zu den kannibalistischen Überfällen und dem Vernichtungskrieg der Kasakela-Schimpansen mit ihren prägnanten Passagen vorwiegend in jenem Sinne, als sie es dem erzählenden primatologischen Ich ermöglichen, die Ereignisse im Rahmen vorgeformter Kategorien zu deuten und darzustellen und damit epistemisch zu nutzen. Darin zeigt sich auch eine Verarbeitungsstrategie für den Umgang des primatologischen Subjekts mit dem Phänomen primatischer Gewalt. Dass die Ereignisse eine stark affektive und verstörende Wirkung haben, wird bei Goodall deutlich hervorgekehrt. Diese „newly recorded“ (92) Verhaltensformen „,changed for ever my view of chimpanzee nature“ (92), schreibt Goodall wie bereits erwähnt als Resümee der Vorfälle im Kapitel „War“" in Through a Window:

For so many years I had believed that chimpanzees, while showing uncanny similarities to humans in many ways were, by and large, rather , nicer' than us. Suddenly I found that under certain circumstances they could be just as brutal, that they also had a dark side to their nature. And it hurt. (92)

In diesem „And it hurt“ steckt eine epistemologische Verletzung ebenso wie eine narzisstische Kränkung. Goodalls Forschungsergebnisse hatten zuvor jahrelang ein optimistisches Bild von Schimpansen als Art gezeichnet; nun muss dieses Bild wesentlich revidiert werden, zum Teil durch die Beobachtungen anderer. Zudem sieht sie hier Gewalt zwischen Individuen, die sie 12 Jahre lang in einem engen Beziehungsgeflecht angeordnet und beobachtet hatte. Dies sind Individuen, die Goodall gut kennt, denen sie Namen verliehen hat, die sie offen als ihre ,Freunde ${ }^{210}$ bezeichnet, mit denen sie sich identifizieren kann. Besonders die Überfälle auf Goliath - ,The third victim was, for me, the most tragic of all [...]. It was none other than my old friend Goliath.“ (89) - und Madam Bee - „I was deeply shocked when the next victim was [...] Madam Bee. I suppose I should have been prepared for this, knowing of the brutal attacks on stranger females. But Madam Bee was not a stranger [...].“(90) - gehen Goodall affektiv sehr nahe.

\footnotetext{
${ }^{210}$ Vgl. van Lawick-Goodall, Jane: My Friends, the Wild Chimpanzees. Washington, DC 1967.
} 
Goodall stellt die Verarbeitung des neuen Wissens als inneren Kampf dar: „For several years I struggled to come to terms with this new knowledge" (92). Anzeichen dafür ist ihr das nächtliche Wirken einer Art traumatischen Bildspeichers:

Often when I woke at night, horrific pictures sprang unbidden to my mind - Satan cupping his hand below Sniff's chin to drink the blood that welled from a great wound on his face; old Rudolf, usually so benign, standing upright to hurl a four-pound rock at Godi's prostrate body; Jomeo tearing a strip of skin from Dé's thigh; Figan, charging and hitting, again and again, the stricken, quivering body of Goliath, one of his childhood heroes. And, perhaps worst of all, Passion gorging on the flesh of Gilkas's baby, her mouth smeared with blood like some grotesque vampire from the legends of childhood. (92)

Dies alles sind Bilder, die aus den vorherigen Schilderungen der Ereignisse ausgespart wurden, die jedoch von hier an mehrfach in eben dieser Detailtreue wieder auftauchen, so schon im wissenschaftlichen Werk The Chimpanzees of Gombe von 1986: Unter dem Kapitel „Territoriality“ (503 ff.) werden die Angriffe nicht nur einzeln und ausführlich im Text dargestellt, sondern auch reichhaltig in einem exemplarischen „Word Picture“ (502) vor Augen geführt. Zudem werden sie ergänzt durch eine tabellarische Aufschlüsselung der Überfälle auf „stranger females by Kasakela males" aus dem gleichen Zeitraum: ${ }^{211}$ nach Opfern, anwesenden Tätern und Täterinnen, Dauer, Wunden, einer Beschreibung des Vorfalls unter der Kategorie „Comments“, der Schwere des Übergriffs (,Level') und den Beobachtenden. In dieser Informationsfülle werden noch mehr Details der Beobachtungen zutage gefördert; die Darstellung der obigen Gewaltakte ist ausführlicher als Goodalls Schilderung in Through a Window. Die dort aufgelisteten Bilder des Schreckens finden sich schon hier: „As he crouched, Satan grabbed him by the neck and sucked blood from his nose“ $(C G, 510)$; ,[o]ne or more of the aggressors tore skin from Dé's legs with their teeth" $(C G, 508)$. Goodalls Verstörung lässt sich in The Chimpanzees of Gombe auch am Bildmaterial ablesen. Zwischen Bildern von ,Patrouillen" finden sich hier zwei Bilder, die durch ihre Unterschriften hervorstechen: „Goliath being groomed by Jomeo, one of the males who later helped to kill him“ (509) und „Mike grooms Goliath, who was seen in peaceful association with Kasakela males only five months before their savage assault" (505).

In Through a Window wird die persönliche Dimension dieser Beobachtungen und die Qualität des Wissens um die schimpansische Gewalt thematisiert. Dass dies mit den Forschungsmemoiren von 1990 nicht abgeschlossen ist, zeigt sich daran, dass neun Jahre später in Reason of Hope in einem „The Roots of Evil“ (111 ff.) betitelten Kapitel fast dieselbe Schilderung nächtlicher Albbilder wiederzufinden ist:

${ }^{211} C G$, S. 495 ff.; für die kannibalistischen Überfälle findet sich Entsprechendes unter dem Kapitel „Aggression“, S. $354 \mathrm{f}$. 
For months I struggled to come to terms with this new knowledge. Often I awoke at night with horrific pictures of violence in my mind: Passion, looking up from the tiny dead body of Gilka's infant, blood smearing her lips; Satan cupping his hand to collect and drink the blood that ran from Sniff's wounded face; Faben twisting Godi's broken leg, round and round. And Madam Bee lying hidden under the vegetation, slowly dying of her terrible wounds, while her ten-year-old daughter tried to comfort her, gently grooming her and keeping the flies away. (118)

Durch das Miterleben (oder Erzähltbekommen) der Gewaltakte wird ein neues Subjekt kreiert - nicht mehr allein das Subjekt der Beobachtung in der Feldforschung, sondern eines, das diese Subjektivität mit der (epistemischen, fingierten oder authentischen) Zeugenschaft zu vereinbaren hat. ${ }^{212}$ Es fällt Goodall in den verschiedenen Forschungsmemoiren schwer, von der Bedeutung der neuen Funde zu berichten, ohne auch davon zu berichten, was dieses neue Wissen mit $i h r$ gemacht habe. Diese zwei Dimensionen des Berichts deuten auf jenes Auseinanderfallen von Affekt und Repräsentation hin, das Dominick LaCapra durch Trauma hervorgerufen sieht: „,[O]ne disorientingly feels what one cannot represent; one numbingly represents what one cannot feel." ${ }^{\text {"213 }}$ Goodalls Verfahren gleicht Versuchen einer historiografischen Bearbeitung von traumatischen Erfahrungen: „Working through trauma involves the effort to articulate or rearticulate affect and representation in a manner that may never transcend, but may to some viable extent counteract, a reenactment, or acting out, of that disabling dissociation." ${ }^{\text {"214 }}$ In den Forschungsmemoiren scheint Goodall die Trauma-Bearbeitung durch eine (Re-)Artikulation der affektiven Wirkung, ein Reenactment der Ereignisse, ein , acting out " der Verstörung, aber auch eine genaue Erfassung der Geschehnisse anzustreben. Wie LaCapra in Bezug auf historiografische Repräsentation darlegt, involviert „experience as it bears on understanding [...] affect both in the observed and in the observer." ${ }^{215}$ Für Goodall stellt sich ein ähnliches Problem wie für die historiografische Beschäftigung mit traumatischen Ereignissen. Einerseits nimmt sie die „traumatic experience of others“ ${ }^{\text {‘216 }}$ wahr, deren Gewalterfahrung sie teilweise als Augenzeugin mitansieht. Diese Zeugenschaft erlebt sie ihrerseits als traumatische Erfahrung. Andererseits ist sie in der Verantwortung, das Beobachtete in die Form der wissenschaftlichen Repräsentation zu überführen. LaCapra sieht in dieser Situation der Empfänglichkeit für die traumatische Erfahrung anderer ein „empathic unsettlement“ entstehen, ,which should have stylistic effects or,

\footnotetext{
${ }^{212}$ Dass dies keine leichte Aufgabe ist, zeigt sich, wie Goodall in Reason for Hope angibt, u. a. an dem Widerstand gegen die neuen Ergebnisse innerhalb der Forschungsgemeinde. Goodalls Bericht wird als „merely ,anectodal " and should therefore be disregarded“ aufgefasst. Ihr wird schlicht nicht geglaubt, dass es so gewesen sei, wie sie berichtet. Und dort, wo ihr geglaubt wird, hält man es für ,politisch“ falsch, ,to publish the facts“" (S. 118).

${ }^{213}$ LaCapra, Dominick: Writing History, Writing Trauma. Baltimore/London 2001, S. 42.

${ }^{214}$ LaCapra: Writing History, S. 42.

${ }^{215}$ LaCapra: Writing History, S. 41.

${ }^{216}$ LaCapra: Writing History, S. 41.
} 
more broadly, effects in writing which cannot be reduced to formulas or rules of method." 217 Die Bilder der Gewalt, die Goodall an das Ende ihrer Beschäftigung mit dem Vernichtungszug der Kasakela-Schimpansen stellt, sind ein solcher Überschuss. Er wird produziert durch einen aus dem ,empathic unsettlement' enstehenden Schreibmodus, ,that allows the reader to feel an affective bond with the victim, without allowing for (over)identification with the victim“, 218 wie Emily Koopman ihn beschreibt. Denn in den betreffenden Passagen zum Kannibalismus und zum Schimpansen-Krieg kommen diese Bilder der Gewalt nicht vor. Die Monstrosität der Gewalt wird damit für den ethologischen Bericht eingedämmt, ohne den empathischen epistemologischen Zugang von Goodalls Feldforschung gänzlich zu verstellen. „Empathic unsettlement“, so beschreiben es auch Loren und Mettelmann, „marks distance from what has occurred and, significantly, from what might be termed the victim position, while allowing for empathy in an attempt to understand historical events“. 219

Für Goodall drängen diese bildhaften Pathosformeln der Gewalt als Symptome traumatischer Erfahrung jedoch schließlich gegen die Form des ethologischen Berichts an. Ihre Erwähnung in der Bearbeitung der neuen Wissenserfahrung lässt sich als Prozess eines so therapeutischen wie interpretativen Schreibens deuten: ${ }^{20}$ „I felt strongly it was better to face up to the facts, however unsettling, than to live in a state of denial“" $(R H, 120)$; ,Gradually, however, I learned to accept the new picture" (TW, 92). Wichtig für diesen Prozess scheint wieder eine Strategie im Text: die Relativierung der Gewalt durch eine Verneinung der moralische ,Agency“ der Schimpansen. Der Modus eines ,empathic unsettlement" wird durch die Entlastung der Kasakela-Schimpansen vom Verdacht einer sadistischen Täterschaft unterstützt. Es wird dabei ein entscheidendes Differenzkriterium zwischen Mensch und Schimpanse etabliert: „For although the basic aggressive patterns of the chimpanzees are remarkably similar to some of our own, “ so schreibt Goodall, ,their comprehension of the suffering they inflict on their victims is very different from ours“ (TW, 92). Obwohl sie hier ihre eigenen früheren Befunde zur ,guten“ Seite der Schimpansen-Gemeinschaft - „Chimpanzees, it is true, are able to empathize, to understand at least to some extent the wants and need of their companions"

\footnotetext{
${ }^{217}$ LaCapra: Writing History, S. 41.

${ }^{218}$ Koopman, Emy: Reading the Suffering of Others. The Ethical Possibilities of ,Empathic Unsettlement'. In: Journal of Literary Theory 4/2 (2011), S. 235-251, hier S. 243.

${ }^{219}$ Loren/Metelmann: Irritation of Life, S. 186.

${ }^{220}$ Der Psychoanalytiker Dori Laub konzipiert das Erzählen vom Trauma als epistemischen Schaffensprozess: „The emergence of the narrative which is being listened to - and heard - is therefore, the process and the place wherein the cognizance, the ,knowing ' of the event is given birth to. The listener, therefore, is a party to the creation of knowledge de novo. The testimony to the trauma thus includes its hearer, who is, so to speak, the blank screen on which the event comes to be inscribed for the first time". Laub, Dori: Bearing Witness or the Vicissitudes of Listening. In: Shoshana Felman/Dori Laub. (Hg.): Testimony. Crises of Witnessing in Literature, Psychoanalysis, and History. New York/London 1992, S. 57-74, hier S. 57, Hervorheb. im Original.
} 
(TW, 92) - mit relativieren muss, behauptet sie doch: ,[O]nly humans, I believe, are capable of deliberate cruelty - acting with the intention of causing pain and suffering“" (TW, 92, Hervorheb. im Original). Die auffällige Trennung von ,our und ,their" Verhalten und Gewalt erlaubt eine Distanzierung vom traumatisierenden Schock-Wert der schimpansischen Gewaltszenen. Dass diese Strategie, die Illusion eines arglosen oder unschuldigen, sogar amoralischen Tötens anzubieten, nicht vollständig erfolgreich sein kann, zeigt sich nicht zuletzt daran, dass Goodall in Through a Window ihr Narrativ damit enden lässt, dass die Kasakela-Gemeinschaft nach Auslöschung der Kahama-Gemeinschaft nun ihrerseits von der KalandaGemeinschaft in ihrer Existenz bedroht werden. Hier heißt es bezeichnenderweise durchaus moralisch-rechtlich wertend: ,retribution was at hand“ (92).

\subsubsection{A (His-)Story of Violence: Primatographie der Gewalt}

„,YOU WILL BE KILLED!' [...] But killing was the reason we were in Africa“ (1). So programmatisch beginnen der Primatologe Richard Wrangham und der Wissenschaftsjournalist Dale Peterson ihr Demonic Males. Wrangham war selbst in Gombe zur Zeit der Schimpansenkriege als Nachwuchsforscher zugegen und nimmt dies Jahrzehnte später als Anlass für eine evolutionäranthropologische Theoretisierung menschlicher Gewalt. ${ }^{221}$ In Demonic Males wird diese Anwesenheit in Gombe als Authentizitätsbeleg genutzt, so wenn Wrangham sich nach der Aufzählung von Goodalls Verdiensten selbst ins Spiel bringt: „I arrived at Gombe in 1970, just as the north-south community division was beginning to be recognized“ (12). Von da an wird das Ich der Observation stark betont: „I followed chimpanzees from dawn to dusk [...]. I noted how [...]. I came to see [...]. I learned where $[\ldots]$. I heard them [...]“ (13), und schließlich: „Comparing notes with my colleagues from the hot, exciting days of that year of discovery, I came to share with them a new view of male chimpanzees as defenders of a group territory, a gang committed to the ethnic purity of their own set" (14). In der populärwissenschaftlichen Metastudie ${ }^{22}$ werden anekdotische Beobachtungen in szenischer Darstellung aus primatologischen und sozialanthropologischen Studien und

\footnotetext{
${ }^{221}$ Siehe auch Wrangham, Richard: The Goodness Paradox. The Strange Relationship Between Virtue and Violence in Human Evolution. New York 2019.

${ }^{222}$ Dass es sich hier um ein Buch handelt, das bei allem wissenschaftlichen Anspruch für eine breitere Öffentlichkeit verfasst ist, zeigt sich am deutlichen Reportagestil, der in die theoretischen Überlegungen einführt, und der detailreichen Darstellung von Gewaltakten, die aus den Texten anderer Primatolog/innen übernommen wurden, sowie an zahlreichen epistemischen ,Zeugenaussagen“ (vgl. z. B. DM, S. 3 ff.) und der direkten Anrede der Leserschaft: „Imagine being battered by five heavyweight boxers and you have an idea how Godi might have felt" (DM, S. 5); „Imagine it, then. The larger group, all or nearly all adult males, settles into a journey, stopping now and again to listen and look and rest. [...] They rest. Several climb trees. All are silent. All face the neighboring range. To the west, Lake Tanganyika sparkles through leafless trees“ (S. 15). Teilweise werden auch kursiv ,Gedanken“ der Schimpansenfiguren eingefügt, um die Eindrücklichkeit zu erhöhen.
} 
kultursoziologische Ansätze zusammengeführt. Ziel dieser Zusammenführung ist: „By following the lines of human ancestry back toward our common ancestry with the great apes, we were looking for patterns of behaviour that would offer, so we believed, clues to a profound and disturbing mystery of the human species" (4 f.). Indem Wrangham und Peterson die Ergebnisse zur Gewalt bei Menschenaffen nutzen, um Gewalt nicht nur beim Menschen, sondern explizit und ausschließlich beim männlichen Menschen biologisch herzuleiten, erzählen sie in Roland Borgards' Definition nicht nur eine „Lebenschrift des Menschen als Gattungswesen“" ${ }^{223}$ sondern schreiben mit ihrem „Apes and the Origins of Human Violence" (so der Untertitel der Studie) eine Primatographie männlicher Gewalt.

Der ,ground zero ' für Wranghams und Petersons Theorie biologischer männlicher Gewalt sind die „Kahama killings“ (21) in Gombe: für die Autoren ,a shock and a stimulus to thought" auf dem Weg zur Theorie vom „killer ape“ (21). Dieser ,Killeraffe“ ist ein Männchen: „This notion of the violent male seems reasonable to anyone familiar with crime statistics, and explains why we can't find paradise on earth" (108f.). Der männliche ,Killer" ist bei Wrangham und Peterson Zuspitzung und Ausgangsgedanke zugleich. Mit dieser Maskulinisierung der primatischen Gewalt ebnen Wrangham und Peterson die Vielfalt, die sich in Goodalls Form-Pluralität abbildet, ein. Es ist weder zufällig noch unproblematisch, dass als Auslöser und Paradigma für ,primatische Gewalt die ,Kahama killings“ und nicht etwa die „cannibalistic attacks“ (TW, 67) ausgewählt wurden. Tatsächlich tauchen die kannibalistischen Übergriffe - Gewaltakte, die von Weibchen an Weibchen und deren Nachwuchs ausgeübt werden - in Demonic Males nicht einmal auf. Um eine biologische Fundierung männlicher, primatischer Gewalt - mit Ausnahme und Gegenentwurf der Bonobos - herzustellen, müssen die von Goodall zusammengetragenen Beobachtungen umgeformt bzw. ausgedeutet werden. Dass Gigi, ausgerechnet ein Weibchen im Östrus, bei etlichen der Überfälle auf fremde Weibchen an den Gewalthandlungen aktiv - und in Goodalls Schilderung enthusiastisch - teilnimmt, wird bei Wrangham und Peterson relativiert: „The only female with them was Gigi. Childless and tough, she wouldn't slow them down" (5, Hervorheb. MS). Gigi erscheint hier nicht wie bei Goodall als sexuell aktives (,fully pink“, $T W, 84$; „cycling“, $C G$ 497), emphatisch an der Gewalt partizipierendes Mitglied der ,raids', sondern als abgehärmtes Anhängsel, das zumindest die Gruppe nicht aufhält. Dass teilweise auch andere Weibchen bei den Überfällen zugegen sind, ${ }^{224}$ wird in der Formulierung ,all, or nearly all adult males“ geradezu versteckt. Dass der biologische Bezug primatischer Vorfahren und -formen auf

\footnotetext{
${ }^{223}$ Borgards: Primatographien, S. 364.

${ }^{224} \mathrm{Vgl}$. CG, S. 495 ff. Die neueste primatologische Feldforschung kennt durchaus noch mehr Fälle tödlicher Gewalt unter Schimpansen und anderen Affen, an der Weibchen entweder aktiv beteiligt sind, oder die sogar allein von diesen ausgeübt wird. Vgl. Zuberbühler, Klaus: Die Sprache der Affen. Vortrag im Rahmen der Collegium-generale-Ringvorlesung Menschen und andere Primaten. Universität Bern 2016.
} 
das Kulturwesen Mensch problematisch ist, darauf verweist bereits Borgards in seiner Poetik der Primatographie. Ergänzend betont die neuere Gewalt(literatur) forschung, dass sich die kulturelle und soziale Einbettung von Aggression, Wut und Gewalt gerade in Bezug auf das Geschlecht nicht umgehen lasse. ${ }^{225}$ Wrangham und Peterson sind sich dieser Problematik durchaus bewusst und versuchen, die Einwände aus den Postcolonial Studies, den Gender Studies und der Kulturanthropologie vorwegzunehmen, besonders die eines postmodernen konstruktivistischen Feminismus (111 ff.). Sie befürworten die Biologisierung von Verhalten als Teil einer ,new philosophy [...], an evolutionary brand of feminism that sees the emergence of patriarchy as an intimate part of human biology“ (125). Wrangham und Peterson propagieren damit einen , evolutionären Feminismus ‘, der

would remove our inhibitions about examining animal behaviour as a technique for thinking about human behavior. They [Vertreter/innen dieser Richtung] would insist that people can think about the evolutionary pressures that elicit rape, for example, or rather forms of violence, without necessitating any absurd pronouncement that because rape is ,natural ' it is in any way forgivable. [...] Any behaviors can still be studied as biological phenomena, regardless of how unpleasant they are. (125)

Dies ist keine leichte Aufgabe, wie man am argumentativen Aufwand feststellt, den die Autoren betreiben müssen. Der strategische Umgang mit den von Goodall aufbereiteten und formal unterschiedlich gestalteten Daten wird zu diesem Zweck ergänzt durch eine Temperamentenlehre der Geschlechter: Das Kapitel „A Question of Temperament" (108 ff.) arbeitet sich an den möglichen Einwänden und ihren Erwiderungen ab, um die Grundthesen des Buches noch einmal herauszuarbeiten:

[T]he suggestion that chimpanzees and humans have similar patterns of violence rests on more than the claim of universal human violence. It depends on something more specific - the idea that men in particular are systematically more violent. Violent by temperament. (108, Hervorheb. MS)

Damit ist das Hauptargument des hier angewendeten Ansatzes ein emotionales. Die Leitfrage für Wranghams und Petersons Untersuchung ist: „What gives our own species such temperamentally violent males?" (126, Hervorheb. MS).

In den Forschungsmemoiren Goodalls ist der Begriff ,Temperament" nicht von Bedeutung. In der Entwicklungspsychologie und der Ethologie jedoch ist das Konzept des Temperaments ein Versuch, Emotionalität zu typisieren und in

\footnotetext{
${ }^{225}$ Elana Gomel verweist in ihrer Studie von Narrativen der Täter/innen-Subjektivität und des ,violent sublime“ darauf, dass ,,[v]iolence is a human activity that may be freely chosen by both male and female subjects. [...] Most cultures [...] have circumscribed female physical violence by an array of taboos. The indisputable fact that a majority of violent offenders are men testifies to the cultural gendering of self-transcendence as masculine rather than to any innate proclivity of women to pacifism“. Gomel: Bloodscripts, S. 209. Collins verweist auf einen regulären Anteil an weiblichen Gewaltformen und in ähnlicher Linie wie Gomel auf Beobachtungen zu Gewalt bei Kindern. Vgl. Collins: Violence, S. 467.
} 
speziesübergreifenden Zusammenhängen zu biologisieren. Temperament scheint in diesem Kontext bei vorsprachlichen Wesen als Äquivalent zur Persönlichkeitslehre eingesetzt zu werden. Wrangham und Peterson setzen hier eine eigene Anmerkung:

Temperament, as we define it, is the emotional element of personality. It is a system of emotional systems; in other words, temperament consists of an individual's emotional reactions to situations in the real world. The idea also includes predictability. Individuals may have predictable reactions to a given set of circumstances. Temperament varies between individuals, but it is also possible to think about the general or average temperament of a sex. Likewise, it's possible to talk about the temperament of a species, in the sense of an average of individual temperaments in that species. (108)

Temperament ist in dieser Definition ein Instrument geworden: Als zugleich der emotionale Teil der Persönlichkeit und Übersystem der Emotionalität umfasst das Temperament die individuelle emotionale Interaktion mit der Welt und garantiert gleichzeitig eine Vorhersehbarkeit emotionaler Reaktionen. ${ }^{226}$ In ihrer Reflexion einer emotionalen Beteiligung am Denken und der Entscheidungsfindung, wie sie mittlerweile affektphilosophisch Konsens ist, entwickeln Wrangham und Peterson so unter der Herausformung von individuelle Differenzen einebnenden Spezies-Profilen (Schimpansen, Menschen, Bonobos) eine Interpretation, der sie den Begriff Temperament auferlegen. So schließen sie aus der Zusammenführung von a) „Men come physically armed for aggression“ und b) ,they look emotionally primed to pursue high status“ ein „male temperament“ (193). In diesem Sinne ist das Temperament ideales Hilfskonstrukt, um primatische Gewalt zu maskulinisieren, sie - unter Aussparung vergleichbarer weiblicher Beispiele - zu homogenisieren, und sie - darin zeigt sich der Erfolg von Goodalls Verhaltenslehre - zu normalisieren.

Wo Jane Goodalls Texte auf die primatische Gewalt durch ,irritierende“ Formenvielfalt in der Darstellung und Interpretation reagieren, damit aber die Komplexität des Themas mitabbilden, bieten Wrangham und Peterson ein homogenes Narrativ als Theorie der Gewalt an, das einen verengten, wenn nicht gar

\footnotetext{
${ }^{226}$ In diesem Sinn ist Temperament zugleich ,the inherited, early appearing tendencies that continue throughout life and serve as the foundation of personality" und eine Art Ersatzwort in der Tierforschung, um bei Tieren den Begriff ,Persönlichkeit" zu vermeiden, aber dennoch individuelle Wesensunterschiede herausstellen zu können. Freeman, Hani D./Gosling, Samuel D.: Personality in Nonhuman Primates: A Review and Evaluation of Past Research. In: American Journal of Primatology 72 (2010), S. 653-671, hier S. 654. Dass Temperament auch wie bei Wrangham/Peterson angekündigt auf Spezies und Geschlechter als Ganzes angewendet wird, zeigt die neuere Forschung, die nicht nur ,the temperamental profiles of species in the hominoid clade" zu charakterisieren, sondern auch herauszufinden versucht, ,how species differences in ecology may shape differences in temperament“. Herrmann, Esther/Hare, Brian/Cissewski, Julia/ Tomasello, Michael: A comparison of temperament in nonhuman apes and human infants. In: Developmental Science 14/6 (2011), S. 1393-1405, hier S. 1393.
} 
teilweise ideologischen Fokus auf die Ergebnisse der Feldforschung richtet. ${ }^{27}$ Goodall versucht sich mit der Einsicht zu trösten, dass einerseits die Gewalttäter/ innen unter den Gombe-Schimpansen ihrerseits von der Nachbargemeinde bedroht werden und so Vergeltung erfahren; andererseits hofft sie darauf, dass den Schimpansen das Bewusstsein für das zugefügte Leid fehlt. Wrangham und Peterson bieten dafür mit ihrem Entwurf eines friedlichen Bonobo-Matriarchats eine Versöhnung mit dem von ihnen kreierten biologischen Imperativ männlicher Gewalt an. Der „Gentle Ape“ (200) zeichnet sich bei Wrangham und Peterson durch einen „threefold path to peace“ (204) aus, der sich - immer in Abgrenzung vom Schimpansen - durch ,reduced levels of violence in relations between the sexes, in relations among males, and in relations between communities“ (205) auszeichne. Entscheidend ist für die Autoren dabei „female power“ (207), ein weibliches „,network of support and security“ (210), ,diverse sexuality“ (213) und eine Verringerung von „male coalitionary skills“ (219). Sie kommen in ihrer Betrachtung der Bonobos in Zentralafrika und deren Evolutionsgeschichte zu dem Schluss, auf dem Weg vom Schimpansen zum Bonobo hätten Männchen ,their demonism“ (219) verloren. Daher biete die Spezies ,the message that it didn't have to be this way, that there is room on the earth for a species biologically committed to the moral aspects of what, ironically, we like to call ,humanity ': respect for others, personal restraint, and turning aside from violence as a solution to conflicting interests“ (230). ${ }^{228}$ Dieser Entwurf beinhaltet ein Dreieck, das wieder die menschliche Sonderrolle betont, indem es die Mehrdimensionalität der menschlichen Spezies folgendermaßen umreißt: „Temperament tells us what we care about. Intelligence helps us generate options. And wisdom can bring us to consider outcomes distantly“ (258). Letztlich bestätigt sich hier Hannah Arendts Vorbehalt gegenüber der ,Naturalisierung von Gewalt: „I fear there lurks behind these newest , discoveries" the oldest definition of the nature of man - the definition of man as the animal rationale, according to which we are distinct from other animal species in nothing but the additional attribute of reason". 229

\footnotetext{
${ }^{227}$ Wrangham/Peterson sehen den von ihnen propagierten ,Evolutionsfeminismus “ durch Primatologinnen wie Patricia Adair Gowaty, Sarah Blaffer Hrdy, Meredith Small und Barbara Smuts vertreten, die ebenso wie die Autoren von Demonic Males Anhänger/innen von Richard Dawkins Selfish-Gene-Theorie sind. Das 1996 erschienene Buch von Wrangham/Peterson lässt sich in einem mittlerweile vorherrschenden wissenschaftlichen Paradigma verorten, das kulturelle Erklärungsansätze bedingt zulässt, aber grundsätzlich einen umfassenden biologistischen Ansatz für menschliches Verhalten verfolgt. Zum ideologische Potenzial von Dawkins' Evolutionstheorie vgl. Abschn. 4.2.

${ }^{228} \mathrm{Vgl}$. in ähnlicher Linie de Waal, Frans: The Bonobo and the Atheist: In Search of Humanism Among the Primates. New York 2013.

${ }^{229}$ Arendt: On Violence, S. 62 f.
} 


\subsection{Traurige Tropen: Trauer und Melancholie in primatologischer Form}

After all, if someone is lost, and that person is not someone, then what and where is the loss, and how does mourning take place? ${ }^{230}$

Die Langzeitforschung an Affen bringt ein Phänomen mit sich, das selbst dort, wo nicht, wie im vorangegangenen Abschnitt diskutiert, andere Affen dafür verantwortlich sind, gravierende Einschnitte in der Forschung bedeuten kann. Wie es Christophe Boesch und Hedwige Boesch-Achermann objektivierend ausdrücken: „Each chimpanzee had an expected probability of being attacked by a leopard every 3.3 years and of being killed by a leopard within 18 years". ${ }^{231}$ Dass dem Tod von (Forschungs-)Tieren Bedeutung zukommt, zeigen alle hier untersuchten Texte. Bei den Boeschs ist es eine statistische; bei Toshisada Nishida, dessen weibliche Untersuchungstiere nach und nach alle von einem Löwen gerissen werden, hat der Tod der Tiere auch eine forschungspraktische Bedeutung:

Over two years, I learned of the deaths of three female chimpanzees with whom I had developed relationships over 20 years: Chausiku, Ndilo, and Wantendele. [...] The motivation and power to go on conducting research on female chimpanzees, as I had done since 1979, no longer resided within me. I forever stopped the systematic study of adult female chimpanzees. ${ }^{232}$

Die Bedeutung des primatischen Sterbens kann darüber hinaus auch ganz ,persönlich“ empfunden werden. Robert Sapolskys A Primate's Memoir steuert narrativ auf das letzte Kapitel „The Plague“ (275 ff.) zu, in dem er von einer Tuberkulose-Epidemie unter den Affen berichtet. Diese rafft zahllos Paviane dahin; ein ,problem whose story I am not yet ready to tell“ (221), wie er zuvor schreibt. Als Sapolsky dann in seiner Erzählung soweit ist, auch diese Geschichte erzählen zu können, wird sie mit einiger Gravitas und einem Hinweis auf den Stil und die Form des Erzählens eingeleitet:

I have decided that it is time to tell how my baboons ended. I have tried throughout this book to give some attention to the style of writing, to try to shape some of these stories. Here I will not try. Things unfolded in an odd, unshaped way. [...] These are not a crafted balanced set of events, and the telling of them will not be particularly crafted either. (267)

Sapolsky kündigt mit dieser Präambel für die zu schildernden Ereignisse eine formale Irritation an: Der Bericht vom Sterben seiner Paviane weiche von

\footnotetext{
${ }^{230}$ Butler, Judith: Precarious Life. The Powers of Mourning and Violence. London/New York 2004, S. 32.

${ }^{231}$ Boesch, Christophe/Boesch-Achermann, Hedwige: The Chimpanzees of the Taï Forest: Behavioural Ecology and Evolution. Oxford 2000, S. 27.

${ }^{232}$ Nishida, Toshisada: The Chimpanzees of the Lakeshore. Natural History and Culture at Mahale. Cambridge u. a. 2012, S. 83.
} 
der erzählerischen Gestaltung der in den Forschungsmemoiren unterbreiteten Geschehnisse ab. Die Ankündigung des vermeintlich ,not particularly crafted“ stößt jedoch wenig später im Text auf Sapolskys Auseinandersetzung mit der besonderen Ausgestaltung von Gedenkworten für Verstorbene. Denn der tierliche Tod und die Trauer ${ }^{233}$ werden in A Primate's Memoir dezidiert formal ausgestaltet. Die Form, die Sapolsky für die Beschreibung der Epidemie und die Darstellung seiner Trauer findet, folgt einer Adaption seiner jüdisch-orthodoxen Wurzeln (14 f.), die er bereits in der Benennung der Primaten und seiner Kapitel geltend gemacht hat:

And the plague took David.

And Daniel.

And Gideon.

And Absalom.

And the plague took Manasseh, who died writhing in front of a laughing crowd of staffers at the lodge.

\footnotetext{
${ }^{233}$ Unter Trauer möchte ich hier mit Reiner Sörries gemeinhin die Reaktion auf einen Verlust und mit Petra Strasser „ein[en] schmerzliche[n] Affekt, ein[en] Primäraffekt des Menschen in Bezug zur Welt und in seinem Bezug zum anderen Subjekt“ verstehen, der als „ein Moment seiner Subjektwerdung, seiner Individuation“ wirken kann. Strasser, Petra: Trauer versus Melancholie aus psychoanalytischer Sicht. In: Wolfram Mauser/Joachim Pfeiffer (Hg.): Trauer. Würzburg 2003, S. 35-52, hier S. 39; Sörries, Reiner: Herzliches Beileid. Eine Kulturgeschichte der Trauer. Darmstadt 2012, S. 11. Als kulturelles Gefühl ist dieser Affekt laut Simone Winko ,überkodiert“, d. h. er wird überdurchschnittlich häufig und in einer ungewöhnlich differenzierten Zeichensprache vermittelt und unterliegt stark kulturellen Gefühlsnormen. Vgl. Winko, Simone: Kodierte Gefühle. Zu einer Poetik der Emotionen in lyrischen und poetologischen Texten um 1900. Berlin 2003, S. 354; Anderson, Inga: Bilder guter Trauer. Neue Sichtbarkeiten der Trauer in der Psychologie, Philosophie und Fotografie. München 2018, S. 34 ff., 92 ff.

Wie Sigmund Freud bereits in „Trauer und Melancholie“ diskutiert, muss es sich bei dem den beiden titelgebenden affektiven Phänomenen zugrunde liegenden Verlust nicht immer um eine geliebte Person handeln, auch „Abstraktionen wie Vaterland, Freiheit, ein Ideal“ oder, wie Nishidas Zitat zeigt, eine Forschungsmöglichkeit können betrauert werden. Freud, Sigmund: Trauer und Melancholie. In: Freud, Sigmund: Psychologie des Unbewußten. Frankfurt a. M. 2000 (Studienausgabe, Hg. von Alexander Mitscherlich/Angela Richards/James Strachey, Bd. 3), S. 193-212, hier S. 197. Gesine Krüger hat in ihrer Beschäftigung mit Renato Rosaldos „Grief and a Headhunter's Rage" bereits danach gefragt, was persönliche Trauer für das wissenschaftliche Arbeiten bedeuten und methodisch bewirken kann. Siehe Krüger, Gesine: Kopfjäger: Trauer und andere Gefühle in der Wissenschaft. In: Belinda Davis/Thomas Lindenberger/Michael Wildt (Hg.): Alltag, Erfahrung, Eigensinn. Historisch-anthropologische Erkundungen. Frankfurt a. M./ New York 2008, S. 105-120.

Dass Trauer nicht unbedingt ,als zweifelhaftes Privileg der Menschen“ (Liebsch, Burkhard: Revisionen der Trauer. In philosophischen, geschichtlichen, psychoanalytischen und ästhetischen Perspektiven. Weilerswist 2006, S. 109) gelten muss, impliziert nicht nur bereits Darwin: Darwin: The Expressions of The Emotions, S. 135 f., 167. In der Primatologie gibt es immer wieder Anlass zur Diskussion von Trauer bei Tieren, u. a. auf Basis der Feldforschung. So schreibt z. B. Smuts vom Verhalten einer Primatenmutter nach dem Tod ihres Nachwuchses und schließt ab mit den Worten: „We cannot be sure that baboons mourn the loss of their friends, but I think it would be premature to claim that they do not". Smuts, Barbara B: Sex and Friendship in Baboons. New York 1985, S. 231. Im Folgenden, wenn zur Unterscheidung nötig, als SF geführt; Seitenangaben im Text.
} 
And the plague took Jesse.

And Jonathan.

And Shem.

And Adam.

And Scratch.

And the plague took my Benjamin. (301)

Diese Passage steht formal durch die Versform, die Namensnennung und die in Bezug auf Manasseh genannten Todesumstände dem antiken Epitaph nahe. ${ }^{234}$ Die durch ihren Anaphern-Einsatz biblisch anmutende Auflistung der Opfer der Tuberkulose-Epidemie verdeutlicht jedoch vor allem das Ausmaß an Verlust durch die schiere Häufung, die den Rahmen eines Epitaphs sprengen muss. Sapolskys Auseinandersetzung mit Formen der Trauer verdeutlicht denn auch die Schwierigkeit, über tierliches Sterben zu schreiben und das Ringen darum, tote Tiere angemessen zu würdigen:

I write these words years later and I still have not found a Prayer for the Dead for the baboons. As a child, when I believed in the orthodoxy of my people, I learned the Kaddish. Once I said it in stunned, mechanical obeisance to my tradition at the open grave of my father, but it glorifies the actions and caprices of a god who does not exist for me, so that prayer does not come for these baboons. I have been told that in primate centers in Japan, Shinto prayers are offered to honor the monkeys that have been killed, and that the prayers are hybrids of the prayer for a dead animal offered by the successful hunter and the prayer for a dead enemy offered by a successful soldier. But even though I stalk these animals with my blowgun and I quicken at a darting, I swear that I have never been their hunter and they have never been my enemy. So that prayer does not come for these baboons. In a world already filled with so many words of lamentation, no words have come to me. And instead, these baboons only remain as ashes in my head. With the ashes of my father's dementia and my science that moved too slowly to help him. With the ashes of my ancestors in the death camps. [...] With the ashes of the rats dead in my lab. With the ashes of my depressions and my bad back that aches more each year. With the ashes of the hungry Masai children who watch me now as I type, wondering if they will be fed here today. (301, Hervorheb. MS)

The tidal waves of AIDS in Africa and desertification and war and hunger make my particular little melodrama seem self-indulgent and small potatoes, a tragedy for a whitey comfortable and privileged enough to be sentimental about animals on the other side of the globe. But still, I miss those baboons. (302, Hervorheb. MS)

Sapolsky bewegt sich hier durch ein Spektrum an Traditionen, den Tod und die Toten zu würdigen - verschiedene Totengebete (Kaddisch, shintoistische Gebete), die Lamentatio und sogar die Tragödie. In dieser Reflexion auf das die Form des Trauerausdrucks bestimmende Verhältnis zwischen Toten und Lebenden findet er so doch wieder eine Form des Schreibens und der Trauer über seine toten Paviane: Indem er

\footnotetext{
${ }^{234}$ Sie verzichtet jedoch z. B. auf die dafür typische glorifizierende Lobrede auf den/die Verstorbene/n und hat an dieser Stelle m. E. eine andere Funktion als schlicht epitaphios logos zu sein. Vgl. Hagenbichler, Elfriede: Epitaph. In: Gert Ueding (Hg.): Historisches Wörterbuch der Rhetorik. Bd. 2. Tübingen 1994, S. 1306-1312.
} 
sie problematisiert, kommen die Worte zu ihm. Und diese Worte gleichen durch die formalisierte Wiederholung in der Anapher sowohl einem rhythmisierten TotenGebet $^{235}$ als auch einer als ,Asche' stilisierten Form des Nachrufs ${ }^{236}$ - auf seinen dementen Vater, auf die in den Konzentrations- und Vernichtungslagern ermordeten Juden, auf die Ideale der eigenen Forschung, auf die eigene Gesundheit und auf die Armen dieser Welt. Nicht zuletzt wird eben dies ein Nachruf auf die toten Tiere, jene des Labors und jene unter Sapolskys Freilandpavianen.

Mich interessiert im Folgenden die Form, die die Worte über Verlust annehmen: Welche , literarische(n) ${ }^{6}$ Form(en) nimmt das tierliche Sterben im Genre der primatologischen Forschungsmemoiren an, mehr noch, wie werden tote Forschungstiere und abstraktere Verluste betrauert und wozu dient die formale Darstellung und thematische Einbettung?

Wie sich bereits bei Sapolskys Reflexion von Trauergebeten zeigt, gibt es eine reichhaltige Verbindung von Trauer und Genre. In ihrer Studie zu Trauer und Genre hat Desirée Henderson auf ,the large number of literary genres inspired by loss“ hingewiesen: „[D]eath is an event that calls for ritualistic and formal genres, particularly those that promise to restore order in the face of the rupturing force of loss“. ${ }^{237}$ Im Folgenden soll die These vertreten werden, dass sich in den Forschungsmemoiren der Primatologie die beiden großen, mit Verlust verbundenen Affektkomplexe der Psychoanalyse und der Literaturgeschichte - Trauer und Melancholie - jeweils in einer eigenen Form abbilden: Die Trauer um individuelle Affen als normativer Verarbeitungsprozess des Verlustes formiert sich im Nachruf, bzw. der Eulogie (Abschn. 3.4.2). Der Schwund ihres Habitats und der drohende Verlust der Spezies selbst wird dagegen in einer Art betrauert und angemahnt, die als unabschließbare Trauerarbeit die Form einer melancholischen Klage annimmt (Abschn. 3.4.3). Beides sind in diesem Kontext, so wird dabei aufzuzeigen sein, politische Formen von Trauer und Melancholie. Bevor es um diese beiden Formen im Text geht, soll jedoch ein Blick auf deren chronologische, affektive und strukturelle Voraussetzung geworfen werden: die Darstellung des tierlichen Sterbens in den Forschungsmemoiren (Abschn. 3.4.1).

\subsubsection{Nature Morte: Stillleben des Sterbens}

„Never in my life have I felt closer to drowning in anger, felt more poisoned, more lost in a corrosive sense of betrayal. I [...] withdrew into my fury [...] obsessing

\footnotetext{
${ }^{235}$ Der Anaphern-Einsatz ruft die ,mechanical obeisance to my tradition“ (Hervorheb. MS) auf, die Sapolsky mit dem Kaddish für Trauernde verbindet, und die bereits in der anaphorischen Struktur der vorherigen Passage der Auflistung der Pavian-Toten anklingt.

${ }^{236}$ Das Asche-Motiv lässt hier einerseits eine u. a. mit Paul Celans Gedicht Todesfuge (1948) tradierte Metonymie für die jüdischen Opfer der Shoah mitschwingen, hat aber andererseits durchaus einen materiellen Bezug zu den Pavian-Toden in Kenia (vgl. Abschn. 4.3).

${ }^{237}$ Henderson, Desirée: Grief and Genre in American Literature, 1790-1870. Burlington/Farnham 2011, S. 4.
} 
over fantasies of vengeance“ (294), schreibt Sapolsky in A Primate's Memoir. Der Tod der vertrauten Forschungstiere bietet den primatologischen Autor/innen Anlass, ,legitimerweise' über ihre eigene Emotionalität zu schreiben. ${ }^{238} \mathrm{Wie}$ bereits an anderer Stelle diskutiert, ereilt Dian Fossey angesichts der Ermordung des Gorillas Digit die Erfahrung von Weltverlust (vgl. Abschn. 2.4.2.3). Als Jane Goodalls Schimpansengruppe von einer Polio-Epidemie erfasst wird, schildert sie diese Episode in In the Shadow of Man als Tage mit einer ,nightmare quality“ (219): „[T]hose few months were the darkest I have ever lived through“ (218). Der Tod David Graybeards ist später ,the saddest loss“ (267). Biruté Galdikas ist in Reflections of Eden bis zur Redundanz hin stark affiziert vom Tod einzelner Orang-Utans:

After Barbara died, I cried all night. I tenderly wrapped her in my best sarong before we buried her (215, Hervorheb. MS).

Tony's death, only two months after Barbara's, devastated me. I should have been prepared, but I wasn't. Irrationally, I had kept hoping that he would recover; irrationally, I felt I had failed him. (216, Hervorheb. MS)

Nothing in my life to that point devastated me more than Cara's, Carl's and Cindy's deaths. [...] Cara's loss was traumatic. It was like watching helplessly as a relative slowly dies of cancer. (251, Hervorheb. MS)

Als die Pavianin Peggy an einem gebrochenen, infizierten Bein dahinsiecht, sieht sich auch Shirley C. Strum im affektiven Ausnahmezustand: „At first I did not know if I should try and save her [...]. But this was Peggy, and my emotions dictated my actions. [...] [W]hy should I pretend that life was normal when it wasn't?" (AH, 202, Hervorheb. MS). Hier kommt eine affektive Dringlichkeit zum Ausdruck, die auch formal ein- und umgesetzt wird. ${ }^{239}$

\footnotetext{
${ }^{238}$ Dies mag darin wurzeln, dass Trauer nicht allein als ein ,privativer[r] Zustand des Seins“ gilt. Liebsch, Burkhard: Trauer als Gewissen der Geschichte? In: Ders./Jörn Rüsen (Hg.): Trauer und Geschichte, Köln u. a. 2001, S. 15-62, hier S. 16. Sie ist von derart großer (und vielfältiger) emotionaler Intensität, dass sie, wie Strasser hervorhebt, ,deutliche affektive und somatische Veränderungen“ hervorruft, motivationale Handlungen überlagert und sogar als „traumatische Emotion aufgefasst werden" kann. Strasser: Trauer versus Melancholie, S. 40. Vgl. auch Renato Rosaldos Beschreibung seiner viszeralen Verlusterfahrung angesichts des toten Körpers seiner im Feld verunglückten Frau, Rosaldo: Grief.

${ }^{239}$ Wie die Trauer-Forschung diskutiert, ruft Trauer eine affektive Äußerungsnotwendigkeit hervor: „Die Trauer ringt um Ausdruck, aber auch mit ihm, um nicht von ihm erdrückt oder konterkariert zu werden“. Liebsch: Revisionen der Trauer, S. 110, Hervorheb. im Original. Liebsch fragt in seiner Diskussion des Konnexes von Geschichte und Trauer daher zugespitzt: „Kann es überhaupt Trauer geben, die nicht zum Ausdruck kommt? Bedarf sie nicht, um wirklich Trauer sein zu können, der Äußerung - ohne ihr jemals ganz entsprechen zu können?“'. Liebsch: Trauer, S. 16. Karl Heinz Bohrer nennt Trauer gar „ein Problem des Ausdrucks, und das heißt der Form“. Bohrer, Karl Heinz: Historische Trauer und Poetische Trauer. In: Burkhard Liebsch/Jörn Rüsen (Hg.): Trauer und Geschichte. Köln u. a. 2001, S. 111-127, hier S. 111.
} 
Im Modus des erzählenden Berichts der primatologischen Forschungsmemoiren ist der Fokus auf dieses eine, sterbende Individuum nicht unüblich. An diesen Stellen tritt jedoch eine fühlende Ich-Erzählinstanz verstärkt in den Vordergrund und gewährleistet damit eine Vermittlung des emotionalen Eindrucks, den das Sterben des tierlichen Anderen hervorruft. Es zeigen sich individuelle, Verarbeitungsweisen' des Sterbens der Anderen. So schreibt Goodall in Through a Window vom Sterben Melissas, einer Schimpansin, die nach dem Tod ihres Kindes ihren Lebenswillen verloren zu haben scheint (,After the death of her infant, Melissa seemed to lose the will to live“ 148), wie folgt:

By evening, Melissa was alone. One foot hung down from her nest and every so often her toes moved. I stayed there, sitting on the forest floor below the dying female. Occasionally I spoke. I don't know if she knew I was there or, if she did, whether it made any difference. But I wanted to be with her as night fell. I didn't want her to be completely alone. As I sat there the quick tropical dusk gave place to darkness. The stars increased in number and twinkled ever more brightly through the forest canopy. There was a distant panthoot far across the valley, but Melissa was silent. Never again would I hear her distinctive hoarse call. Never again would I wander with her from one patch of food to the next, waiting, at one with the life of the forest, as she rested or groomed with one of her offspring. The stars were suddenly blurred and I wept for the passing of an old friend.

The next morning I watched as Melissa took her last, laboured breath; her body shuddered, then relaxed. All around, during those last hours, the branches had swayed and rustled as youngsters played while elders fed on the lucious fruits. In the midst of life there is death. This was an appropriate setting for Melissa's passing, allegorical in its portrayal of the inevitable cycles of nature. I was deeply moved, but my tears were over. Melissa had indeed known a hard life, with many misfortunes, but she had lived fully and, for much of that time, had clearly enjoyed living. She had attained a high rank. And, most importantly, she had left a solid succession. (148 f., Hervorheb. im Original)

Jane Goodall nutzt hier erstens die Passage, um ihre Anteilnahme am tierlichen Sterben - als „Melissa's passing“ euphemisiert - zu bekunden und sich selbst in der Rolle einer Sterbebegleiterin darzustellen. ${ }^{240}$ Zweitens wird ihr die Szene wörtlich zur allegorischen Darstellung des ,Kreislauf des Lebens', dem sie mit dem kenntlich gemachten Gebets-Vers ${ }^{241}$ einen Hinweis auf ihre eigene religiöse Prägung

\footnotetext{
${ }^{240}$ Hier ließe sich wohl Jacques Derridas Vorwurf des Narzissmus der Hinterbliebenen anbringen: die Versuchung, sich der Toten zum eigenen Zweck zu bedienen, mit dem Schreiben von und über die Toten politischen oder persönlichen Gebrauch vom Tod des anderen zu machen, und sei es, das eigene Schicksal im Tod der anderen zu reflektieren. Siehe Brault, Pascale-Anne/Naas, Michael: Einleitung: Mit den Toten, den Toden rechnen. Jacques Derrida und die Politik der Trauer. In: Jacques Derrida: Jedes Mal einzigartig, das Ende der Welt. Hg. von Peter Engelmann. Wien: Passagen 2007 (frz. 2001), S. 17-55, hier S. 23 ff.

${ }^{241}$ Media vita in morte sumus - In the midst of life we are in death ist der Beginn eines gregorianischen Chorals und findet sich dank seiner Popularität sowohl als Lutherische Hymne (EG 518, vgl. Herbst, Wolfgang (Hg.): Komponisten und Liederdichter des Evangelischen Gesangbuchs. Göttingen 1999, S. 204 ff.) als auch unter der Rubrik „At the buriall of the dead“ im Book of Common Prayer der Church of England (The Book of Common Prayer And Administration of the Sacraments: And other Rites and Ceremonies of the Church of England, London 1640, ohne Seitenzahlen). Aus diesem findet es seinen Weg in Leichenreden und -musik, z. B. Henry Purcells Music for the Funeral of Queen Mary (1695), und wird weiter popularisiert.
} 
im Umgang mit Natur und Tod vorgibt. Die mediative und wohl auch meditative Charakteristik dieser Art von Erlösung im Religiösen zeigt sich besonders im Vergleich mit Biruté Galdikas, die angesichts des Todes der Cara-Familie ebenfalls mit dem ,cycle of nature" konfrontiert wird. Dies gibt Galdikas Anlass, den Titel ihrer Forschungsmemoiren, Reflections of Eden, einzulösen und das in der Anspielung ,Garden of Eden“ angelegte primatologische Heilsnarrativ ${ }^{242} \mathrm{zu}$ dekonstruieren, indem sie ein Natur-Verständnis entromantisiert:

\begin{abstract}
We would never be quite the same again. Our idyllic Garden of Eden existence had been shattered. We had been living in a delusion brought about by our youth, our isolation, and our North American upbringing. Like so many Westerners, especially the flower children of the 1960 s, I had been partially seduced by the ,naturalistic fallacy“. Nature was pure and noble, beautiful and bright. Nature produced happy endings. In travelling to the tropical rain forest, Rod and I were fulfilling our generation's dream of ,going back to nature', returning to the Garden of Eden. But gardens are made by humans, to please human sensibilities. To maintain a garden, one must keep nature at bay - weeding, pruning, spraying, watering, fencing. A garden is Nature tamed, domesticated, civilized. In the beginning, there was no garden, there was only Eden. Our original home was not a garden but a wild place, where Nature reigned. I was learning that Nature clean and pure was also Nature brutal, ruthless, savage. (252)
\end{abstract}

Der Kontrast mit Galdikas' Reflexion der Naturidealisierung zeigt, wie stark Goodalls Hang zur naturromantischen Allegorisierung den häufig brutalen Tod, den die Affen in den meisten Forschungsmemoiren sterben, formal im religiös inspirierten Stillleben einhegt. Goodalls Naturromantik ist geprägt von ihrer anglikanischen Erziehung und Auffassung. Schon in ihren zweiten Forschungsmemoiren, Through a Window, wird ihr die ästhetische Naturerfahrung in der „trance-like mood“(9) zur spirituellen Erfüllung:

\begin{abstract}
As I stood quietly in the pale sunshine, so much a part of the rainwashed forests and the creatures that lived there, I saw for a brief moment through another window and with other vision. It is an experience that comes, unbidden, to some of us who spend time alone in nature. The air was filled with a feathered symphony, the evensong of birds. I heard new frequencies in their music and, too, in the singing insect voices, notes so high and sweet that I was amazed. I was intensely aware of the shape, the colour, of individual leaves, the varied patterns of the veins that made each one unique. [...] And I was utterly filled with the peace, which passeth all understanding ${ }^{\text {. }}$ (8 f.)
\end{abstract}

Es ist wieder das schließende Zitat aus der King-James-Bibel, das die suggestiv-beruhigende Wirkung von Goodalls religiösem Naturverständnis unterstreicht: „And the peace of God, which passeth all understanding, shall keep your hearts and minds through Christ Jesus“ (Philippians 4:7). In Reason for Hope wird der Konnex von romantischer Natur und religiöser Erfahrung noch expliziter. Dort heißt es zu ihrer Beschäftigung mit Bibelstellen und Naturgedichten in ihrer Kindheit:

\footnotetext{
${ }^{242}$ Vgl. auch Kilchör, Fabienne/Lehmann, Jörg/Liebal, Katja/Lubrich, Oliver: Diesseits von Eden. Paratexte und Bilder in der Primatographie. In: Scientia Poetica 22/1 (2018), S. 151-179.
} 
Clearly at that time, I was starting to feel myself as part of a great unifying power of some kind. Certain things caused feelings of such profound happiness that tears would come to my eyes [...]. At moments such as those, I felt strongly that I was within some great spiritual power - God. $(R H, 30)$

Goodalls Naturbeschreibung greift häufig Allegorien auf, die auf einen Subtext der Trauer verweisen, die wie eingefroren im Naturbild erscheint. So erinnert ihre Beschreibung der Nacht, in der Melissa stirbt, an Matthias Claudius' „Abendlied“"243:

But I wanted to be with her as night fell. I didn't want her to be completely alone. As I sat there the quick tropical dusk gave place to darkness. The stars increased in number and twinkled ever more brightly through the forest canopy. [...] [A]t one with the life of the forest $[\ldots]$. The stars were suddenly blurred and I wept for the passing of an old friend. $(T W, 148)$

Anders als Sapolskys einleitende Verschriftlichungsproblematik und seine, wie noch zu zeigen wird, angesichts des Massensterbens von Pavianen gehegte Rachephantasie (vgl. auch Abschn. 4.3.3), zeigt Goodalls Beispiel eine Verarbeitungsweise auf, in der der erregte emotionale Zustand, der mit Macht um Ausdruck ringt, auf tradierte rhetorische und motivische Formen trifft, den Tod als Teil sowohl des Naturkreislaufs als auch einer spirituellen Rite-de-Passage abzubilden und zu normalisieren.

\subsubsection{Toter Tiere Trauerreden: der primatologische Nachruf}

Was Jane Goodalls Through a Window mit den anderen primatologischen Forschungsmemoiren teilt, ist die Abrundung der Passage durch eine Beurteilung von Melissas Leben, die Nennung ihrer Verdienste und ihrer Hinterbliebenen. Bei den für die Forschungsarbeit ausschlaggebenden Affen arbeitet die Darstellung des Sterbens derart auf eine besondere Form hin: Das tierliche Sterben und die erzählte Existenz dieses Tieres werden mit dem Nachruf, einer Totenrede für die Verstorbenen, vollendet. ${ }^{244}$

\footnotetext{
${ }^{243}$ „Der Mond ist aufgegangen,/ Die goldnen Sternlein prangen/ Am Himmel hell und klar./ Der Wald steht schwarz und schweiget,/ Und aus den Wiesen steiget/ Der weiße Nebel wunderbar. [...]“. Asmus: Abendlied. In: Voss, Johann Heinrich (Hg.): Musen-Almanach für 1779. Hamburg 1778, S. 184-186.

${ }^{244}$ Angesichts der Fülle an unterschiedlichen rhetorischen Trauerkonventionen, die in der Forschung voneinander abgegrenzt werden können - Henderson allein zählt auf: „elegy, funeral sermon, funeral oration, eulogy, obituary, epitaph, tragedy, tribute, lament, dirge, requiem, monody, thenody, encomium, panegyric, osequies, thanatopsis, and memento mori“ - soll hier unter Hinweis auf den modernen Sprachgebrauch und das moderne Genre, dessen sich die primatologischen Texte bedienen, schlicht vom Nachruf (im Verständnis der Begräbnisrede ,eulogy“ oder des schriftlichen Nekrologs ,obituary') die Rede sein, wie ihn Henderson versteht als ,a standard variety of memorial in either spoken or written form, through which the deceased and his or her achievements are remembered and celebrated“. Henderson: Grief and Genre, S. 4, Hervorheb. im Original, S. 47.
} 
Diese primatologischen Nachrufe teilen zentrale Charakteristika des Genres in der Darstellung der Verstorbenen. Diese sind nach Henderson: „The use of biography and historiography to define character, the employment of hyperbole, and the mimetic link between speaker and subject". ${ }^{245}$ Zudem ist das zentrale rhetorische Instrument des Nachrufes die Idealisierung. Am primatologischen Nachruf lassen sich all diese Charakteristika nachvollziehen: Die Biographie der individuellen Tiere wird ebenso genutzt wie die ,Historie" des Forschungszusammenhangs; Persönlichkeit, Verhalten und Verdienste werden hyperbolisch zugespitzt und letztlich sind die derart betrauerten Tiere idealisierte Repräsentant/innen der Spezies sowie Symbole einer besonderen Beziehung zwischen Forschenden und Beforschten. In Fosseys Gorillas in the Mist titt so Digit nach seiner Ermordung vor allem als tapferer Krieger auf. Sein Nachruf ist, wie bereits erläutert (vgl. Abschn. 2.4.2), eine Schilderung seines heroischen Verhaltens und seiner Bedeutung für die Forschende, sowie seiner Symbolkraft für das Projekt Artenschutz. Auch der für Jane Goodall so wichtige Schimpanse David Graybeard wird nach seinem Ableben in In the Shadow of Man entsprechend gewürdigt. Besonders seine Bedeutung für Goodall und ihr Forschungsanliegen im ,Drama of Touch` (vgl. Abschn. 2.2) wird dabei hervorgehoben:

David had been the first chimpanzee to accept my presence and the first who permitted me to approach him closely. Besides providing me with my early exciting observations of meat-eating and tool-using and thus helping to ensure the availability of further funds for my research, he had been the first to visit my camp, the first to take a banana from my hand, the first who permitted a human hand to touch him. (SM, 267, Hervorheb. MS)

Neben der Betonung der instrumentellen Rolle für Goodalls Forschungsprojekt, geht es Goodall jedoch auch darum, David Graybeards Persönlichkeit und ihre Beziehung zu ihm (als Hinterbliebene) zu besprechen:

David, with his gentle disposition, who permitted a strange white ape to touch him. To me it represented a triumph of the sort of relationship man can establish with a wild creature, a creature who has never known captivity. In those early days I spent many days alone with David. [...] Sometimes, I am sure, he waited for me - just as he would wait for Goliath or William - for when I emerged, panting and torn from a mass of thorny undergrowth, I often found him sitting, looking back in my direction [...]. (SM, 268, Hervorheb. MS)

Für Shirley Strum ist Peggys Tod ,a symbol, an indication that the future would be very different from the past“ $(A H, 201)$. Auch ihr geht es darum, die Bedeutung der Verstorbenen für sie als Hinterbliebene aufzuführen: „Throughout the ten years I had studied Peggy and her family, she had been very special to me" $(A H, 201)$. Wie Goodall zeichnet auch Strum ein Bild von Peggys Persönlichkeit und Wesen:

There was so much to remember about Peggy: her way of handling infants, and of grooming, which was special and unique to her because she had only one good eye; her calm, almost regal demeanor, in such striking contrast to that of her daughters and granddaughters. What stood out most in my memory was her social skill, her intelligent sophistication [...]. (AH, 203, Hervorheb. MS)

${ }^{245}$ Henderson: Grief and Genre, S. 4. 
Und auch hier geht es um die Bedeutung der verstorbenen Pavianin für die Forschung bzw. die Forscherin:

Peggy had given me many crucial insights about baboons. Her life had taught me what was important and what was trivial. But the most meaningful lesson was much more personal: Peggy had taught me that you can have strong emotions, such as the special attachment I felt for her, and still do good science. The two were not, as I had once thought, mutually exclusive. In fact, they could be related. Peggy's death was the end of an era. It took place exactly ten years from when I began my first study of Pumphouse. The baboons and I had come a long way, but we still had a long way to go. ( $A H, 203$, Hervorheb. MS)

Peggys Lehre für Strum, so fasst der Nachruf zusammen, ist jene der produktiven Vereinbarkeit von Emotionen und Wissenschaft. Abgerundet wird im Nachruf auf Peggy diese Darstellung von Persönlichkeit und Bedeutung des individuellen Tieres für die Forschung durch die Interpretation des Todes als Symbol: als Wendemarke (,end of an era“, ,,a symbol, an indication“) der Forschungsarbeit.

Diese Textpassagen in den Forschungsmemoiren sind jene „occasional forms, designed to address specific deaths with an immediacy and individuality" ${ }^{246}$ wie sie Desirée Henderson im Hinblick auf die amerikanische Literatur im ,funeral sermon“ und der Eulogie findet: ,[B]oth genres employ complex literary techniques to represent the identity of the deceased, as well as situate him or her within overarching narratives“. ${ }^{247}$ Der Nachruf stellt zudem als literarische Form die rhetorischen Strukturen bereit, ,through which grief could be equally represented and soothed“. ${ }^{248}$ Nachrufe sind also erstens eine literarische Textstrategie für eine Art Trauerarbeit, d. h. für jenen Prozess, der nach Sigmund Freud nach der Realitätsprüfung, welche ergibt, „daß das geliebte Objekt nicht mehr besteht“", „,alle Libido aus ihren Verknüpfungen mit diesem Objekt“ unter dem Sträuben des Ich dagegen abzieht, ,,unterdes die Existenz des verlorenen Objekts psychisch fortgesetzt“ wird, bis nach der Vollendung der Trauerarbeit das Ich ,wieder frei und ungehemmt" ist. ${ }^{249}$ Sie bieten den traditionsreichen und rhetorisch gefestigten Rahmen eines Trauer-Genres, um die eigene Emotionalität der Verlusterfahrung zum Ausdruck zu bringen, indem die individuelle Beziehung zum verstorbenen Anderen benannt und damit der entstandene affektive Verlust für den Vortragenden identifiziert wird. Dass dies in Bezug auf die Trauer um Tiere rechtfertigungsbedürftig erscheint, zeigt Sapolskys Thematisierung des Anrechts auf die Verlusterfahrung durch den Tod , seiner' Paviane: Neben dem verstorbenen Vater, neben

\footnotetext{
${ }^{246}$ Henderson: Grief and Genre, S. 16.

${ }^{247}$ Henderson: Grief and Genre, S. 16.

${ }^{248}$ Henderson: Grief and Genre, S. 4.

${ }^{249}$ Freud: Trauer und Melancholie, S. 198 f.; vgl. auch Strasser: Trauer versus Melancholie.
} 
den ermordeten Vorfahren, neben der Masse an menschlichem Tod scheinen die toten Paviane unwichtig - und doch affiziert ihr Tod den Forscher gewaltvoll. ${ }^{250}$

Zudem ist die von Henderson angesprochene mimetische Verbindung an diesen Nachrufen auf Affen auffällig: als ,sense of connection between the living and the dead [...] [t]his link works to establish an identity for the speaker that builds upon the identity being articulated for the deceased“" ${ }^{251}$ Die Primatolog/innen rufen im Nachruf noch einmal das individuelle Tier als Stellvertreter/in der Art, als exemplarisches Tier der ethologischen Forschung, und sich selbst - durch die Assoziation zwischen dem/der Verstorbenen und dem nachrufenden Ich - als Forscher/in und fühlendes Individuum auf. Diese Assoziation verweist bereits auf den politischen Gehalt der Eulogie: ${ }^{252}$ Mit dem Nachruf spricht die Primatologie sowohl vom toten Tier als auch von der eigenen Trauer um dieses Tier. Der Nachruf als Ausdruck eines Trauerprozesses um ein individuelles Tier dient somit zweitens der Erzeugung einer Betrauerbarkeit des Tieres.

Neben der im Affekt selbst begründeten politische Sprengkraft der Trauer spielt der Nachruf als Instrument des Politischen (ob als Eulogie oder als ,Obituary') besonders in der Ethik Judith Butlers eine große Rolle. ${ }^{253}$ Butler sieht die Trauer selbst bereits nicht als private Emotion, sondern als Grundlage eines „sense of political community“" ${ }^{254}$ Dass Trauer nicht unbedingt einen persönlichen Verlust nur indiziere, darauf weist ähnlich auch Burkard Liebsch mit Emmanuel Levinas hin: „vielmehr bezeugt sie - im Fall des Namenlosen deutlicher noch als im Fall des Nahestehenden - die Weigerung, je einen Tod, den Tod von ,irgendeinem', als schlicht ,bedeutungsloses', d. h. hier: absolut indifferentes Faktum hinzunehmen.“255 Für Butler ist die Möglichkeit oder Unmöglichkeit, zu trauern oder

\footnotetext{
${ }^{250}$ Dass das Affiziertsein vom Tod des Tieres so konstitutionell wie problematisch für die Tier/ Mensch-Differenz ist, verhandelt u. a. Sternad in seiner Kritik des heideggerschen Diktums vom Verenden (statt des Todes) des (weltarmen, da Todes-unbewussten) Tieres. Sternad setzt mit Derrida und Agamben gegen Heidegger: „Der Tod des Tieres spricht unmittelbar an und stellt keineswegs nur ein neutrales Verenden dar. Der Mensch gewahrt und antizipiert den Tod des Tieres, vergleicht den menschlichen mit dem tierischen Tod - und der Tod des Tieres ist in jedem Fall bedeutungshaft. Er geht nicht spurlos vorbei und verstärkt sich ebenso wie der menschliche Tod durch die Nähe und die Geschichte dieser Nähe“. Sternad, Christian: Den Tod als Tod vermögen. Zum Tod des Tieres aus phänomenologischer Sicht. In: Tierstudien 5 (2014), S. 47-58, hier S. 57.

${ }^{251}$ Henderson: Grief and Genre, S. 57.

${ }^{252}$ Vgl. Loraux, Nicole: The Invention of Athens. The Funeral Oration in the Classical City. New York 2006. Liebsch sieht in antiker Tradition (Antigone) die Trauer als genuin politisch, besonders in ihrem Hang zum Exzess: „Keine soziale oder politische Gemeinschaft kann überleben, der die kulturelle Bändigung exzessiver Trauer nicht gelingt“". Liebsch: Trauer, S. 17.

${ }^{253} \mathrm{Vgl}$. von Redecker, Eva: Zur Aktualität von Judith Butler. Einleitung in ihr Werk. Wiesbaden 2011, S. $117 \mathrm{f}$.

${ }^{254}$ Butler: Precarious Life, S. 22.

${ }^{255}$ Liebsch: Trauer, S. 22.
} 
betrauert zu werden, hochpolitisch: Nicht betrauerbar zu sein, heißt auch, verletzt und getötet werden zu können - ohne dass es zählt: ${ }^{256}$

If violence is done against those who are unreal, then, from the perspective of violence, it fails to injure or negate those lives since those lives are already negated. But they have a strange way of remaining animated and so must be negated again (and again). They cannot be mourned because they are always already lost or, rather, never ,were', and they must be killed, since they seem to live on, stubbornly, in this state of deadness. Violence renews itself in the face of the apparent inexhaustibility of its object. ${ }^{257}$

Butler spricht hier von den nicht-amerikanischen Opfern des ,War on Terror", den unbetrauerten, ungezählten ,Casualties of War', ja sogar von Terrorist/innen selbst, deren Tod nicht als Verlust betrauert werden darf. Diese Passage Butlers lässt sich jedoch leicht und mit Adornos Hinweis darauf, dass die Gleichgültigkeit dem Tod des Tieres gegenüber eintrainiert werden müsse, ${ }^{258}$ auf Tiere beziehen: ${ }^{259}$ Auch

\begin{abstract}
${ }^{256}$ Diese Überlegungen fallen in Butlers grundsätzliche, unter dem Fragenkomplex „Who counts as human? Whose lives count as lives? And, finally, what makes for a grievable life?" ausgeführte Kritik an der Rede von der Menschlichkeit. Butler: Precarious Life, S. 20, Hervorheb. im Original. Der Begriff des Menschen ist bei Butler mit einer normativen Verwerfung verschwistert, die ihn allzu leicht in ein Gewaltinstrument umschlagen lässt. Sich in der Kategorie des Menschlichen zu fassen, bedeute immer die Übernahme normativer Abgrenzungen gegen andersartige Leben und Identitäten, somit einer ,schändlichen Unterscheidung'. Vgl. von Redecker, Eva: Zur Aktualität von Judith Butler, S. 113. Verletz- und Tötbarkeit sind entscheidende Kriterien in diesen Abgrenzungsprozessen. Obwohl Butler, wie Keri Weil bereits kritisiert hat, mit den prekären Leben immer mit-menschliche und nicht-tierliche meint, ähnelt ihre Konzeptualisierung jenen Ab- und Ausgrenzungsmechanismen, die Agamben in seiner Skizze der ,anthropologischen Maschine instrumentell macht. Vgl. Weil, Kari: Thinking Animals. Why Animal Studies Now? New York 2012, S. 113 ff.; Agamben, Giorgio: Das Offene. Der Mensch und das Tier. Frankfurt a. M. 2003 (ital. 2002), S. 87 ff. Für eine Kritik von Butlers Konzentration auf ausschließlich menschliches Leben siehe auch Taylor, Chloé: The Precarious Lives of Animals. In: Philosophy Today 52/1 (2008), S. 60-72.
\end{abstract}

${ }^{257}$ Butler: Precarious Life, S. 33.

${ }^{258}$ „Die stets wieder begegnende Aussage, Wilde, Schwarze, Japaner glichen Tieren, etwa Affen, enthält bereits den Schlüssel zum Pogrom. Über dessen Möglichkeit wird entschieden in dem Augenblick, in dem das Auge eines tödlich verwundeten Tiers den Menschen trifft. Der Trotz, mit dem er diesen Blick von sich schiebt - ,es ist ja bloß ein Tier ${ }^{*}-$, wiederholt sich unaufhaltsam in den Grausamkeiten an Menschen, in denen die Täter das ,Nur ein Tier' immer wieder bestätigen müssen, weil sie es schon am Tier nie ganz glauben können“. Adorno, Theodor W.: Minima Moralia. Reflexionen aus dem beschädigten Leben [1951]. Frankfurt a. M. 2003, S. 118.

${ }^{259}$ Vgl. Weil: Thinking Animals, S. 109 ff. Weil versucht in ihrer Butler-Kritik das Problem der Unartikulierbarkeit von ,,[a]nimal death and animal loss“ (S. 110) in der Konstruktion einer Mensch/Tier-Differenz durch eine postmodern-psychoanalytische Differenzierung von Trauer (mourning/grief) und Melancholie zu fassen. In der Folge von Freud und DeCapra sieht Weil mourning/grief als den normativen Verarbeitungsprozess eines Verlustes, melancholia dagegen als das Ergebnis eines Widerstandes gegen den erfolgreichen Vollzug dieses Verarbeitungsprozesses: „a state characterized by an immersion in ambivalence and excess“ (S. 107). Weil stellt fest: ,[N]onhuman animals belong to the constitutive outside of the human, designating the boundary between what or who is and is not grievable according to what or who is or is not 
ihr Tod darf im seltensten Fall offiziell betrauert werden, und da er - gerade beim sogenannten Wildtier - nicht betrauert wird, zählt der Tod nicht, hinterlässt keine Leerstelle und kann unendlich wiederholt und wiederausgeführt werden. ${ }^{260}$

Indem die Primatolog/innen in diesen Texten den toten Schimpansen, Gorillas, Orang-Utans oder Pavianen den Nachruf schreiben, stemmen sie sich gegen diese Bedeutungslosigkeit des individuellen Tieres und seines Todes. „,T]he genre of the obituary“, so Butler, ,where lives are quickly tidied up and summarized, humanized" ${ }^{261}$ setzt das gewesene Leben in ein bestimmtes, der Anerkennung dienendes Narrativ: „If there were to be an obituary, there would have to have been a life, a life worth noting, a life worth valuing and preserving, a life that qualifies for recognition."262 Mit Butlers Überlegungen zu einer Ethik der Trauer(form) lassen sich die primatologischen Nachrufe vor allem sehen als ,the means by which a life becomes, or fails to become, a publicly grievable life, an icon for national self-recognition, the means by which a life becomes note-worthy. "263

Die „power of grief“264 wird in der Textform des Nachrufes als politisches Vermögen sichtbar. Die trauernden Primatolog/innen übernehmen durch den von Henderson als ,association between the speaker and subject [that] enables the

humanized“ (S. 113, Hervorheb. im Original). Als das normativ Nicht-zu-betrauernde (und daher als nicht abschließbarer Verlust) sind Tiere dem Bereich der Melancholie zugewiesen. Aus der Widerständigkeit der Melancholie heraus entsteht dann für Weil wiederum ein subversives Potenzial für den ontologischen Status des Tieres (,,,the animal“ as the living dead“, S. 101) und für eben jene normativen Begriffe von Trauer und Betrauerbarkeit.

${ }^{260}$ Zur Geschichte der Trauer um Tiere vgl. jedoch u. a. Sörries: Herzliches Beileid, S. 182 ff. Dass der Tod von Tieren auch Platz im Rahmen poetologischer Überlegungen hat, zeigt Northrop Frye, wenn er als Gegenstand des Pathos ,,a weakness which appeals to our sympathy“ annimmt und durch die ,inarticulateness of the victim“ verstärkt sieht. Neben Frauen, Kindern und verminderter Intelligenz ist für Frye auch das Tier Pathos-auslösend: „The death of an animal is usually pathetic“. Frye: Anatomy of Criticism, S. 38 ff.

Beispielhaft ist auch Friedrich Hebbels Trauer um das Eichhörnchen Herzi-Lampi-Schatzi, in der es unter anderem heißt: „Wen die Gattung für das Individuum zu entschädigen vermag, der ist gegen jeden Verlust gedeckt; ich kenne keine Surrogate, ich liebe das Individuum, und dies Tier war so einzig, daß es jedermann wie ein Wunder vorkam, und mir wie eine Offenbarung der Natur". Hebbel, Friedrich: Tagebücher 1848-1863. Tagebücher. Vollständige Ausgabe in drei Bänden, Bd. 3. Hg. von Karl Pörnbacher. München 1984, S. 304-309, hier S. 304. Siehe auch Borgards, Roland: Herzi-Lampi-Schatzis Tod und Bobbys Vertreibung. Tierliche Eigennamen bei Friedrich Hebbel und Emmanuel Levinas. In: Michael Rosenberger/Georg Winkler (Hg.): Jedem Tier (s)einen Namen geben? Die Individualität des Tieres und ihre Relevanz für die Wissenschaften. Linz 2014, S. 68-83. Auch Alexander von Humboldt findet den Tod eines Schwarzkopfaffen, den er auf dem Schiff mit sich führt, zumindest bemerkenswert. So notiert er diesen Tod zugleich mit der zoologischen Beschreibung dieses Affen und zeichnet ein Kreuz, wie in Todesanzeigen üblich. Vgl. das „Notizblatt mit einer Beschreibung und der Geschichte des von Humboldt gezeichneten Cacajao-Äffchens, datiert Mai 1800, Carichana am Orinoko“. In: Hahlbrock, Peter: Alexander von Humboldt und seine Welt 1769-1859: Führer durch die Ausstellung im Schloss Charlottenburg, Orangerie. 29. Juni bis 10. August 1969. Berlin 1969, S. 19.

${ }^{261}$ Butler: Precarious Life, S. 32.

${ }^{262}$ Butler: Precarious Life, S. 32.

${ }^{263}$ Butler: Precarious Life, S. 34.

${ }^{264}$ Weil: Thinking Animals, S. 114. 
eulogist to present a narrative of inheritance or replacement, as the eulogist becomes the living figure of the dead" 265 beschriebenen ,mimetic link" gleichsam ein Mandat für die Erinnerung an das individuelle verstorbene Tier. ${ }^{266}$ Dieses Mandat reicht soweit, auch die betreffenden Primatolog/innen zu politischen Repräsentant/innen der Spezies werden zu lassen.

\title{
3.4.3 Anthropozäne Melancholie und primatologische Klage
}

\begin{abstract}
Ein Schleier der Trauer, der tristitia, ist geworfen über die Passage vom homo zum homo sapiens, wie positiv sie auch sein mag. ${ }^{267}$
\end{abstract}

Had I read Lévi-Strauss earlier in my career, I might have been better prepared for what the baboons were doing to my world. $(A H, 158)$

Wenn Shirley Strum schreibt, eine rechtzeitige Lévi-Strauss-Lektüre hätte sie darauf vorbereiten können, wie die Paviane ihre Welt verändern würden, bezieht sie sich auf das letzte Kapitel von Triste Tropique, „Le Retour“. ${ }^{268}$ Darin reflektiert Lévi-Strauss die ethnologische ,déformation professionelle“, „la mutilitation complémentaire à sa vocation“",269 die der Beruf des Ethnographen mit sich bringe: eine doppelte Entfremdungserfahrung. Die Rolle des Ethnographen, so Lévi-Strauss, bestehe schließlich einzig darin, ,de comprendre ces autres au nom dequels il ne saurait agir, puisque le seul fait qu'ils sont autres l'empêche de penser, de vouloir à leur place, ce qui reviendrait à s'identitifier à eux. “270 In seiner eigenen Gesellschaft/Kultur jedoch müsse er, ,renoncera à l'action [...], de peur de prendre position vis-à-vis de valeurs qui risquent de se retrouver dans des sociétés différentes, et donc d'introduire le préjugé dans sa pensée“". ${ }^{271}$ Lévi-Strauss sieht also die Aufgabe darin, die fremden Anderen zu verstehen und verständlich zu machen, ohne jedoch in ihrem Namen - für sie - zu handeln. Denn die ethnologische Fremdheit bietet immer das Problem einer ,falschen Identifikation': An ihrer Stelle denken und handeln zu wollen, verdrängt (ähnlich wie Gayatri Spivak kritisch einwenden

\footnotetext{
${ }^{265}$ Henderson: Grief and Genre, S. 57.

${ }^{266}$ Besonders offensichtlich erscheint das politische Vermögen eines solchen Mandats für die Erinnerung an das individuelle Tier in Fosseys Gorillas in the Mist, wenn Fossey mit Ian Redmond entscheidet, „Digit's slaying“ publik zu machen, und die amerikanische Fernsehjournalismus-Legende Walter Cronkite Digits Tod auf dem Fernsehsender CBS verkünden lässt, siehe $G M$, S. 209.

${ }^{267}$ Steiner, George: Warum Denken traurig macht. Zehn (mögliche) Gründe. Frankfurt a. M. 2006 (engl. 2005), S. 8.

${ }^{268}$ Lévi-Strauss, Claude: Tristes Tropiques. In: Ders.: Euvres. Hg. von Vincent Debaene u. a. Paris 2008, S. 1-445, hier S. 402-445.

${ }^{269}$ Lévi-Strauss: Tristes Tropiques, S. 413.

${ }^{270}$ Lévi-Strauss: Tristes Tropiques, S. 414.

${ }^{271}$ Lévi-Strauss: Tristes Tropiques, S. 414.
} 
würde $)^{272}$ die Anderen aus der Rolle der eigenständigen Akteure. Es kann kein vollständiges, zum Handeln ,für die anderen' ermächtigendes Verständnis geben, denn dies würde die Distinktion von Ich und Anderem einschmelzen: ein fatales ,going native' des Ethnologen, mit dem niemandem gedient sei. Gleichzeitig jedoch entfremdet dieses immer nur annähernde Verstehen der Anderen den Ethnologen von seiner eigenen Gesellschaft, weil er diese und ihre Werte und Strukturen zunehmend relativierend durch die Brille der Ethnologie sehen muss.

Die primatologischen Forschungsmemoiren berichten von vergleichbaren Entfremdungserfahrungen. Strums Bericht von ihren akademischen Konfrontationen ( $A H, 157$ ff.) sprechen Bände über die Entfremdung von der akademischen, viel mehr noch als von der westlichen Kultur. Anders als Lévi-Strauss es hier skizziert, scheuen sich die meisten Primatolog/innen jedoch nicht, im Sinne ihrer Anderen zu handeln, an ihrer Stelle zu denken und zu wollen. Ganz im Gegenteil: Die primatologische Feldforschung ist immer mehr mit der Bedrohung ihres Untersuchungsgegenstandes und dessen Lebensraums konfrontiert, und wie Fosseys hypertrophes Beispiel zeigt, kann die Primatologie kaum anders, als dieser Bedrohung selbst entgegenzutreten. ${ }^{273}$ Die Primatolog/innen ergreifen daher intraund extratextuell nicht nur ein Mandat für die Erinnerung an das individuelle Tier, sondern auch ein Mandat für die Rettung der ,sterbenden Natur'. Es äußert sich im Text in Form einer besonderen Klage, die getrieben scheint von anthropozäner Melancholie: ,[T]rying to hold back the tears, I realized, that I wasn't weeping for Dian or for the mountain gorillas. I was also weeping for chimpanzees, for orangutans, and for a world that is rapidly disappearing" $(R E, 396)$, wie Biruté Galdikas in Reaktion auf die Ermordung Dian Fosseys schreibt.

\subsubsection{Primatologie und Anthropozän}

Schon lang vor der Begriffsprägung des Anthropozäns durch den Atmosphärenchemiker und Meteorologen Paul Crutzer und den Biologen Eugene F. Stoermer fallen in der Primatologie das Bewusstsein von der Zerstörungskraft, die Menschen auf ihre Umwelt und auf andere Arten ausüben, der Abschied von (der Idee) einer ,unberührten' Natur und der Aufruf zum Engagement zusammen. ${ }^{274}$ Überwiegend steuern die Forschungsmemoiren auf Passagen zu, die von der Forschungserinnerung und der Diskussion primatischer Charakteristika und ethologischer Methoden hin zu einer Textform schwenken, die im Dienste des Artenschutzes steht

\footnotetext{
${ }^{272}$ Siehe Spivak, Gayatri: Can the Subaltern Speak? In: Cary Nelson/Lawrence Grossberg (Hg.): Marxism and the Interpretation of Culture. Basingstoke 1988, S. 271-313.

${ }^{273}$ Dies zeigen auch die Beispiele von Robert Sapolsky und Shirley Strum, die sich für ihre Paviangruppen einsetzen. Sapolskys Kampf gegen die menscheninduzierte Tuberkulose-Epidemie droht immer wieder in die Vendetta abzurutschen (vgl. PM, S. 293 ff. sowie Abschn. 4.3.3); Strum siedelt ihre Paviane sogar in ein anderes Gebiet um, damit die Konflikte zwischen Mensch und ,Natur' umgangen werden können, deren Opfer meist die Affen sind. Vgl. AH, S. 205 ff.

${ }^{274}$ Vgl. Crutzen, Paul J./Stoermer, Eugene F.: The Anthropocene. In: Global Change Newsletter 41 (2000), S. 17-18. Die Primatologie partizipiert mit dem Folgenden an einem ökologischen Diskurs, den bereits Heise und Bühler besprechen, vgl. Bühler: Ecocriticism; Heise: Nach der Natur.
} 
und die Form einer Klage annimmt. Ob in George Schallers The Year of the Gorilla, Jane Goodalls zweiten Forschungsmemoiren Through A Window oder in Biruté Galdikas' Reflections of Eden: Trotz der jeweiligen Unterschiede der Forschungsarbeit, der Forschungstiere und des Schreibstils der Autor/innen ähneln sich diese Passagen, auf die sich die Forschungsmemoiren im Zeichen des Artenschutzes zubewegen. Die (An-)Klage im Zeitalter des Anthropozäns beinhaltet jeweils eine Art (Evolutions-)Geschichte der menschlichen Zivilisation, die in den Naturraum drängt, und eine Schilderung von deren Schrecken und deren Auswirkung auf den Untersuchungsgegenstand; sie betrauert das Sterben der belebten und unbelebten Natur und sie schließt mit einem Appell, das Ruder auch im Dienste der eigenen Spezies herumzureißen.

George Schaller befasst sich in The Year of the Gorilla auf mehreren Seiten mit dem Stand der Wildpopulationen in den Gegenden, die er bereist, und kommt zu dem Zwischenfazit: „Whenever man occupies land, the game decreases, for the animals are hunted and their habitat is destroyed. [...] The African population is expanding, pushing farther into the remaining wild-life areas. What can be done to save a remnant?" (73). Das Problem ist für Schaller zunächst ein lokales, das er im Geist seiner Zeit auch als zivilisatorisches betrachtet. Es bedarf für ihn der Schulung Afrikas durch Europa und Amerika, denn: „The preservation of wild life ultimately depedends, of course, on the African himself“ (73); ,one urgent need is to teach the African the value of wild life. We in America can speak from experience of the fate of the game“" (74). Dann wird Schaller jedoch universeller und hebt an zu einer Darstellung dessen, was den Kern der Denkweise vom Anthropozän ausmacht: ${ }^{275}$

\footnotetext{
The whole problem is one of human ecology. Man is conquering the diseases that once kept his population in check, and he is spreading his sway, exterminating other animals and exhausting the soil. With the same mentality that once enabled him to vanquish the lion and the bear, he is trying to subdue nature, sacrificing the eternal for the expedient. The destruction of the earth lies at his whim and cunning, yet he does not realize, does not feel, that he is not separate from but one with plants and animals, rock and water. He is as dependent on them as the protozoan, the tsetse, and the gorilla. By setting himself apart from the ecological community man has become a tyrant of the earth, but a tyrant who surely will fall if he succeeds in winning the struggle for existence. (74)
}

Jane Goodall entwickelt in Through a Window ihre Gedanken anhand einer idyllischen Szene spielender Schimpansen im Wald. Sie betreffen zunächst die Evolution des Menschen aus einer solchen Szene heraus:

In the richness of such a lush environment lived the chimpanzee-like creatures that became the first men. Slowly they evolved. Some became more adventurous and left the forest on excursions into the surrounding savanna, in search of food and new territory.

\footnotetext{
${ }^{275}$ Steffen et al. definieren das Anthropozän ausgehend von einem ,quantitative shift in the relationship between humans and the global environment“. Verantwortlich dafür ist, ,that humankind, our own species, has become so large and active that it now rivals some of the great forces of nature in its impact on the functioning of the Earth system“, zugespitzt sogar: „humankind has become a global geological force in its own right“. Steffen/Grinevald/McNeill: The Anthropocene, S. 843.
} 
[...] They found caves and fire, learned to build dwellings, to hunt with weapons, to talk. And then they became bold and arrogant. They began to hack at the outskirts of the forest itself, bending to their will that which for so long had nurtured them. Today, striding the face of the globe, humans clear the trees, lay waste the land, cover mile upon mile of rich earth with concrete. Humans tame the wilderness and plunder its riches. We believe ourselves all-powerful. But it is not so. (203)

\section{Von dieser Entwicklungsgeschichte der Spezies geht Goodall über zu einer Bilanz der Zerstörung:}

Relentlessly the desert inches forward, gradually replacing the life-sustaining forests with barren und uncompromising harshness. Plant and animal species vanish, lost to the world before we have learned their value, their place in the great scheme of things. World temperatures soar, the ozone layer is depleted. All around we see destruction and pollution, war and misery, maimed bodies and distorted minds, human and non-human alike. (203)

Wie Schaller geht auch Goodall über zur Warnung: „If we allow this desecration to continue we shall, ourselves, be doomed. We cannot meddle so greatly in the master plan and hope to survive“ (203). Zunächst folgt hierauf Hoffnungslosigkeit: „Thinking of this whole terrible picture, the magnitude of our sin against nature, against our fellow creatures, I was overwhelmed. How could I - or anyone - make a difference in the face of such vast and mindless destruction?" (203). Hoffnung gewinnt Goodall, wie in anderen Texten, aus dem Anblick, ihrer' Schimpansen, denn:

Here, at least, was perfect trust between humans and animals, perfect harmony between creatures and their wild environment. [...] Trust. And freedom. I thought of the countless chimpanzees who have lost their forest homes, and of the prisoners in zoos and labs around the world. [...] The will to fight, to fight to the bitter end, flared up. (203 f.)

\section{Goodalls Text endet mit dem Appell:}

The chimpanzees need help now more than before, and we can only help if we each do our bit, no matter how small it may seem. If we don't, we are betraying not only the chimpanzees but also our own humanity. And we must never forget that, insurmountable as the environmental problems facing the world may seem, if we all pull together we have a good chance of bringing about change. We must. It is as simple as that! (204)

Biruté Galdikas schließlich entwickelt am Ende ihres Reflections of Eden und in Zusammenhang mit Fosseys Ermordung ähnliche Gedanken. Die ihren fallen jedoch zu Beginn bereits düsterer aus als Goodalls:

At the heart of Dian's dilemma [Forschung vs. Artenschutz] is the inescapable truth that the battle to save endangered species and their habitats never ends. On the front lines of conservation there are no time-outs, no short-cuts, and few final victories. [...] [S]adly, many conservation , success stories" are Pyrrhic victories, which may lull us into complacency and blind us to the truth of what is actually happening to animal populations and ecosystems in far-off corners of the globe. Or even close to home. (396)

Galdikas führt kurz den aktuellen Stand ihrer Forschungsspezies an: „Once wild orangutans numbered in the hundreds or thousands and roamed throughout Asia. 
Now fewer than thirty thousand remain in the rapidly disappearing tropical rain forests of Borneo and northern Sumatra“ (369). Sie setzt dies in Zusammenhang mit den menschlichen Eingriffen in die Umwelt:

\begin{abstract}
Orangutans face extinction because the tropical rain forests where they live are being cut down for timber, plantations, roads, and permanent agriculture. Every day, between forty thousand and one hundred acres of rain forest are destroyed around the world. The murder of orangutan mothers and capture of orangutan infants for the pet trade is a direct consequence of habitat destruction. Once forest is destroyed, once orangutans become ,homeless', their death warrants are signed.

Along with other endangered species, orangutans are the innocent victims of human population growth, of development schemes, and power struggles, of an insatiable global economy that creates greed but not satisfaction, desire but not happiness. The same global forces that ensnare the mountain gorilla have cost thousands of orangutans their lives. (397, Hervorheb. MS)
\end{abstract}

Auch Galdikas kommt anschließend zur Warnung, dass die Vernichtung der Orang-Utans als stellvertretend für die menschliche Selbstvernichtung betrachtet werden müsse:

With the other great apes, orangutans are among our closest living relatives. More than other species, great apes remind us of our unity with nature. Because we are so closely related to the great apes, scientists often use them as stand-ins for humans in experiments. But we ignore the ,natural experiment" taking place in tropical forests right now. As we watch the great apes slip toward extinction, we are witnessing our own future on an increasingly inhospitable planet. (397)

Galdikas' Appell lautet knapp: „If we take action to save our nearest relatives and their tropical habitats, we are taking the first step toward saving ourselves“ (397).

Das Thema aller drei Autor/innen ist das Artensterben und der Tod der Natur als nicht-modifizierter Lebensraum. Dabei zeigen sich zwar motivische Unterschiede in der Ausgestaltung: Schallers Augenmerk liegt auf dem Wildtier- und Naturverständnis of „the African“ ( $Y G, 73)$ als Zivilisationsfrage und auf dem Menschen als einzigartigem Wesen mit einer ausgeprägten Schattenseite (,sway“, „mentality“, „whim and cunning“, ,a tyrant of the earth“, $Y G$, 74). Jane Goodall sieht ihrer religiösen Grundempfindung folgend in der menschlichen ,,arrogance“ vis-àvis ,the great scheme of things“ und dem „master plan“ eine Sünde (,sin against nature“, „desecration“, TW, 203). Biruté Galdikas’ Wortwahl fällt in den semantischen Bereich des Kampfes und Krieges (,the battle“, „front lines“, „,final victories“, „Pyrrhic victories“, $R E, 396)$ und des gewaltvollen Todes (,murder“, „,death warrants“, $R E, 397)$. Ihre Ausführungen sind dabei entlang einer Kapitalismuskritik gestaltet, die anders als Schaller nicht vom naiven Farmer oder Viehtreiber (,the agriculturalist", ,[t]he pastoralist“, $Y G, 74$ ) her denkt oder mit Goodall die Spezies als ganze der Hybris verfallen sieht. Bei Galdikas sind es globale Entwicklungskonzepte (,development schemes and power struggles“, $R E, 397)$ und ein kapitalistisches System, das Gier erzeugt, ohne Befriedigung zu liefern (,,an insatiable global economy that creates greed but not satisfaction, desire but not happiness“, $R E$, 397). In den ,global forces“ schwingt zwar die Formulierung von der Menschheit als 
,global force' im Anthropozän-Diskurs mit. Bei genauer Betrachtung scheint Galdikas hier aber eher ein globalisiertes Netz der Ressourcen-Ausbeutung im Blick zu haben. Diese Passagen ähneln sich in ihrer Form, Intention und Funktion innerhalb einer Rhetorik des Artenschutzes jedoch so sehr, dass sich von einem eigenen Genre sprechen lässt: der primatologischen Klage.

\subsubsection{Klage und Melancholie}

Diese Passagen sind zunächst in jenem Sinne formal eine Klage, als sie etwas - einen Missstand - beklagen und Ausdruck einer leidvollen Reflexion über einen Verlust sind. ${ }^{276}$ Zwar wird eine Form der Trauerklage, das Lamento, als sprachliche Form diskutiert, in der sich die leidenschaftliche, affekthafte Seite der Trauer artikulieren kann. ${ }^{277} \mathrm{Im}$ antiken Athen ist dies die weibliche Klage als subversives Gegenstück zur institutionalisierten, staatstragenden Form der ,immer stärker zur eulogischen Lobrede tendierenden Leichenrede". ${ }^{278}$ Die primatologische Klage ist aber eben nicht jene vermeintlich dem Affekt ausgelieferte, ,weibliche' und subversive Klage. ${ }^{279}$ Sie ist im Gegenteil kalkuliertes rhetorisches Instrument eines politischen Anliegens: Die Leserschaft soll mittels dieser Klage zum einen durch wohlgewählte Argumentationslinien und katastrophische Bildlichkeit belehrt, zum anderen - und durchaus mithilfe affektiver Ansprache und emotionaler Selbstdarstellung (,I was overwhelmed“, TW, 203; ,trying to hold back the tears“, $R E$, 396; „weeping“, $R E$, 396) - zum Handeln bewegt werden. Die primatologische Klage steht also unter dem Zeichen von Northrop Fryes ,,rhetoric of persuasion to action itself“280 und jener „,großen Geste“

\footnotetext{
${ }^{276}$ Zur Klage als Ausdrucksform eines ,reflexiven Leidens‘ siehe Schmidt, Jochen: Klage. Überlegungen zur Linderung reflexiven Leidens. Tübingen 2011. Schmidt versteht darunter ein Leiden „,nicht allein [...] im Medium der Fähigkeit des Menschen, sich denkend auf Gegenstände im Allgemeinen zu beziehen, sondern näherhin Leiden im Medium der Fähigkeit des Menschen, sich denkend auf sich selbst zu beziehen" (S. 5).
}

${ }^{277} \mathrm{Vgl}$. Anderson: Bilder guter Trauer, S. 109.

${ }^{278}$ Anderson: Bilder guter Trauer, S. 109.

${ }^{279}$ Vgl. auch Marcel Leppers Hinweis auf die ,Gemachtheit‘ dieser Charakterisierung durch die „Disziplinierung der Klage“ u. a. durch Platon, der im Staat unter einem pädagogischen Männlichkeitsideal die „Klagerhetorik aus der politischen Öffentlichkeit in den weiblichen Aufgabenbereich“ verschiebe. Lepper, Marcel: Lamento. Zur Affektdarstellung in der Frühen Neuzeit. Frankfurt a. M. 2008, S. 110. Nicole Loraux weist bereits auf kulturelle Unterschiede der Klagepraktiken auch in der griechischen Antike hin, indem sie die oratorische Toten- und Staatsverherrlichung Athens mit der Trauerpartizipation ganz Spartas im Lamento vergleicht, vgl. Loraux: The Invention of Athens, S. 80.

${ }^{280}$ Frye: Anatomy of Criticism, S. 327. Die primatologische Klage passt sogar genau zu jener Charakterisierung des Pamphlets oder der Rede bei Frye, ,that catches the rhythm of history, that seizes on a crucial event or phase of action, interprets it, articulates the emotions concerned with it, or in some means employs a verbal structure to insulate and conduct the current of history“. Denn ,,[h]ere the repetitions are hypnotic and incantatory, aimed at breaking down customary associations of ideas and habitual responses, and at excluding any alternative line of action“; ,such rhetoric must follow the dialectic of rhetoric: it must have either a rallying point or a point of attack, or both". Die primatologische Klage hat sowohl den ,point of attack ' in ihrer Kritik der menschlichen Zerstörungstendenz als auch den ,rallying point ‘ in ihrem Aufruf zum gemeinsamen Handeln.

${ }^{281}$ Lepper: Lamento, S. 15. 
die auf rhetorische Verfasstheit und Funktionalisierung hinweist, d. h. sie bedienen sich des gebührenden Pathos. ${ }^{282}$ Die primatologische Klage ist - in ihrer Verwandtschaft mit der Totenklage - sowohl den Ausdrucksformen der Trauer wie jenen der Politik verbunden.

Sie ist zudem ohne die spezifische Melancholie des Anthropozäns nicht denkbar: 283 Was die Primatolog/innen hier in den Forschungsmemoiren in der Form der primatologischen Klage darstellen, ist das Bewusstsein eines Verlusts, das sich jedoch dem Abschied vom Verlorenen verweigert. Sigmund Freuds Unterscheidung von Trauer und Melancholie betrachtet Melancholie wie die Trauer als Reaktion auf einen Verlust. ${ }^{284}$ Sie ist jedoch eine Reaktion, in der keine erfolgreiche Trauerarbeit geleistet werden kann. Im Fall des Verlusts der Natur und der Artenvielfalt, die in der primatologischen Klage thematisiert werden, ist das verlorene Objekt zum einen ideell und kann nicht libidinös ersetzt werden - denn das Anliegen der freudschen Klage ist es gerade, den offensiv beklagten Verlust in seinem Prozess aufzuhalten, wenn nicht sogar rückgängig zu machen. Das Subjekt muss so - folgt man Freuds Differenzierung - in Melancholie ,verharren“. Diese Melancholie ist in der Primatologie gepaart mit jenem politischen Gestus des Willens zu utopischen Entwürfen, wie ihn Wolf Lepenies ,den Intellektuellen“ unterstellt: „Der Intellektuelle klagt über die Welt, und aus dieser Klage entsteht das utopische Denken, das eine bessere Welt entwirft und damit die Melancholie vertreiben soll“" ${ }^{285}$ Sie lässt sich zudem auch mit Karl Heinz Bohrers Figur des Abschieds als Reflexionsfigur interpretieren. Denn Bohrer konzipiert den Abschied als „Bewusstseinsform moderner Trauer“, betont darin jedoch weniger „das schmerzliche oder elegische Gefühl angesichts eines erfahrenen Verlustes",286 sondern die Erkenntnis von dessen „strukturell angelegter Vorgegebenheit"“287 Die primatologische Klage offenbart diese strukturell angelegte Vorgegebenheit in ihrer Erzählung einer unweigerlich sich von der ,Natur' emanzipierenden Menschheit. Sie folgt jedoch darüber hinaus Lepenies' Verklammerung von Melancholie und Utopie auch insofern, als sie

\footnotetext{
${ }^{282}$ Lepper sieht in seiner Diskussion der Affektdarstellung in der Frühen Neuzeit die Klage als lamentatio in der Tradition der rhetorischen Struktur der antiken Totenklage (laudatio, lamentatio, consolatio). Sie ist zudem auch durch effektverstärkende Pathosformeln in ihrer Wandlung zu Formen der ,sozialen' oder ,politischen' Klage stark in rhetorischen Handlungszusammenhängen verwurzelt. Vgl. Lepper: Lamento, $108 \mathrm{f}$.

${ }^{283}$ Zur melancholischen Haltung des Anthropozäns siehe auch Bühler: Ecocriticism, S. 154 f. Auch hier ist noch einmal Fryes Schema gültig (wenn auch mit seinem Tadel der „tantrum prose“ voreingenommen), das dieser Form rhetorischen Engagements auch eine zunehmende emotionale Involviertheit mit ihrem Sujet bescheinigt, „,so that what he [the author] exhorts us to embrace or avoid is in part a projection from his own emotional life“. Frye: Anatomy of Criticism, S. 328.

${ }^{284} \mathrm{Vgl}$. Freud: Trauer und Melancholie, S. $199 \mathrm{ff}$.

${ }^{285}$ Lepenies, Wolf: Melancholie und Gesellschaft. Mit einer neuen Einleitung: Das Ende der Utopie und die Wiederkehr der Melancholie. Frankfurt a. M. ${ }^{3} 1998$, S. XXI.

${ }^{286}$ Bohrer, Karl Heinz: Der Abschied. Theorie der Trauer: Baudelaire, Goethe, Nietzsche, Benjamin. Berlin 2014, S. 10.

${ }^{287}$ Bohrer: Der Abschied, S. 10.
} 
den Verlust widersprüchlicherweise gerade in der Hoffnung beklagt, diese Zwangsläufigkeit abzuwenden. ${ }^{288}$ Sie entwirft Szenarien des Untergangs: „If we allow this desecration to continue, we shall, ourselves, be doomed“ (TW, 203); „As we watch the great apes slip toward extinction, we are witnessing our own future on an increasingly inhospitable planet“" $(R E, 397)$. Sie entwirft zugleich aber auch Szenarien utopischer Hoffnung: ,If we take action to save our nearest relatives [...], we are taking the first step toward saving ourselves“ (RE, 397); ,,if we pull together we have a good chance of bringing about change" (TW, 204).

Die primatologische Klage ist damit ein emotionales Instrument der Kritik. ${ }^{289}$ Schon Freud hebt in seiner Betrachtung der Melancholie hervor, Melancholiker/ innen fielen durch die gegen sich selbst gerichtete Kritik auf. Hinter dieser gegen das Ich gerichteten Kritik stecke allerdings Kritik am Anderen, „Vorwürfe gegen ein Liebesobjekt" ${ }^{290}$ oder - eine sehr passende Formulierung für diese Passagen in den Forschungsmemoiren: „Ihre Klagen sind Anklagen“. ${ }^{291}$ Das Liebesobjekt der primatologischen (An-)Klage sind bei näherer Betrachtung aber nicht die vehement verteidigten Affen (denn diese und ihre ,Unschuld' können im Nachruf genuin betrauert werden) - es ist die Menschheit. Die anthropozäne Melancholie ist in viel umfänglicheren Sinn, als Freud dies in seinem Aufsatz konzipiert, eine narzisstische. ${ }^{292}$ Das Konzept des das Holozän als Erdzeitalter ablösenden Anthropozäns ist ein ambivalentes: Ursprünglich zur Klassifizierung im Rahmen der geologische Chronologie entwickelt, wird es nun herangezogen, um den Zustand des Planeten anzumahnen und menschliches Verhalten dafür in die Pflicht zu nehmen. Es baut aber ganz wesentlich zunächst auf einem Ausbau menschlichen Distinktionsbedürfnisses auf: ,Wir' sind die einzige Spezies, die es geschafft hat, den Planeten nicht nur als Schallers ,tyrant of the earth“ $(Y G, 74)$ zu beherrschen, sondern als ,global geological force in its own right ${ }^{“ 293}$ auch umfassend und nachhaltig zu gestalten.

\footnotetext{
${ }^{288}$ Die primatologische Klage bewegt sich im Spektrum von Melancholie und Gesellschaft damit in der Konzeption, die Lepenies anhand von Burton vornimmt - vor dem Einsetzen von Handlungshemmung, Resignation, Weltflucht und Innerlichkeit, wie er es an der bürgerlichen Kultur des 18. und 19. Jahrhunderts nachweist. Sie ist eher dem verschrieben, was Lepenies als das durchaus paradoxe ,melancholische Klima“ der Anthropologie Arnold Gehlens herausarbeitet. Zwar operiert sie mit dem Handlungsaufruf, sie bleibt jedoch „,im Klima der Melancholie die traurige Wissenschaft“. Lepenies: Melancholie und Gesellschaft, S. 253. Vgl. auch, S. 229 ff.

${ }^{289}$ Wie Melancholie als Instrument der Kritik verwendet werden kann, zeigt bereits Judith Butler in ihrer Untersuchung von Subjektwerdung, vgl. von Redecker, Eva: Zur Aktualität von Judith Butler, S. $87 \mathrm{ff}$.

${ }^{290}$ Freud: Trauer und Melancholie, S. 202.

${ }^{291}$ Freud: Trauer und Melancholie, S. 202, Hervorheb. im Original.

${ }^{292}$ Sie nähert sich insofern auch wieder dem Lamento an: „, [T]ears are shed over the dead, of course, but above all they are weeping over themselves“, wie Loraux anhand der Darstellung von Trauerpraktiken in Euripides' Hiketiden feststellt. Loraux: The Invention of Athens, S. $82 \mathrm{f}$. Auf die ego- als anthropozentrische Perspektive der ,Artenelegie' verweist Heise: Nach der Natur, S. 75 .

${ }^{293}$ Steffen/Grinevald/McNeill: The Anthropocene, S. 843.
} 
In diesem Sinn läuft die Rhetorik des Anthropozäns Gefahr, Teil jener Haltung zu sein, die der Primatologe Frans de Waal als ,Anthropodenial ${ }^{\circ 294}$ bezeichnet: Der unbedingte Wille, ,uns' aus der Gesamtheit und Gemeinsamkeit von Lebensformen zu entheben, als Menschheit weiter aufzuwerten, ,uns ' herauszustellen aus der Gemeinsamkeit des Ökosystems, und zum Leitbegriff desselben zu machen. ${ }^{295}$ Das verlorene Liebesobjekt der Primatologie ist das Ideal einer Menschheit, die sich in Einheit mit Natur und Kreatur (,our unity with nature“, $R E, 397$ ) begreift:296 ,perfect trust between humans and animals, perfect harmony between creatures and their wild environment" (TW, 203), wie Goodall schreibt; ein vor-reflektives Zeitalter, ,an Eden that once was ours“, ohne ,seperation between ourselves and nature“ $(R E, 403)$, „our humanity“ (TW, 203) als Teil einer ,ecological community“ $(Y G, 74)$.

Damit partizipiert diese primatologische Klage an einem elegischen Narrativ, wie es Ursula Heise für die vergangenen 200 Jahre in Anschlag bringt: „Die Gesellschaft der Gegenwart, so die Vorstellung, zerstört die Natur, die ein oder zwei Generationen vorher noch intakter, idyllischer und schöner war".297 Die primatologische Klage kann daher auch als Unterform des von Heise identifizierten „Genre[s] der Artenelegie“ ${ }^{298}$ betrachtet werden. In der Artenelegie wird das Artensterben zum „Grundbestandteil des Nachdenkens über die kulturelle Entwicklung, insbesondere über Modernisierungsprozesse“ ${ }^{299}$ in dem sich ein „Unbehagen an der Moderne“300 äußert, und in dem ,charismatische“ Tierarten, die ,synekdochisch für das Ganze stehen und auf umfassendere Krisen im

\footnotetext{
${ }^{294}$ Siehe de Waal, Frans: Are we in anthropodenial? In: Discover 18/7 (1997), S. 50-53.

${ }^{295}$ Konsequenterweise wurde in Vorläufern des Anthropozän-Konzeptes, wie der Idee des „Anthropozoikum“, diese gestalterische Allmacht der Menschheit durchaus positiv bewertet, vgl. Schuster, Jana: Sanftmütige Gärtner, oder: postkatastrophische Kulturation im frühen Anthropozän. Geo- und Bionarrative bei Stifter. In: Gabriele Dürbeck/Jonas Nessehauf (Hg.): Repräsentationsweisen des Anthropozän in Literatur und Medien. Berlin 2019, S. 29-47; sowie Trischler, Helmuth: The Anthropocene. A Challenge for the History of Science, Technology, and the Environment. In: NTM Zeitschrift für Geschichte der Wissenschaften, Technik und Medizin 24 (2016), S. 309-335, hier S. 311.

${ }^{296}$ Zudem nähert sich die Primatologie hier auch Lévi-Strauss' spezifisch ethnologischer Melancholie an. In seiner Rousseau-Lektüre fördert Lévi-Strauss ein Ideal der Menschheit im Naturzustand als theoretisches Modell und Orientierungstypus menschlicher Gesellschaften zutage. An dieser Lektüre von Rousseaus ,L'homme naturel' des Discours sur l'origine et les fondemens de l'inégalité par les hommes zeigen sich Lévi-Strauss' Reformierungsgedanken. Aus der ethnologischen Melancholie wird der Appell geformt, an der Untersuchung der ,Anderen “ ein zukunftsweisendes Menschheitsmodell (wieder-)zufinden. Vgl. Lévi-Strauss: Tristes Tropiques, S. 419 ff.

${ }^{297}$ Heise: Nach der Natur, S. 18.

${ }^{298}$ Heise: Nach der Natur, S. 73.

${ }^{299}$ Heise: Nach der Natur, S. 10.

${ }^{300}$ Heise: Nach der Natur, S. 54.
} 
Umgang mit der Natur hinweisen“, 301 als Symbole fungieren: „Letzten Endes wird also das Tierschicksal durch anthropozentrische Erzählverfahren kulturell bedeutsam, man könnte sagen, es werde zur Metapher für kulturgeschichtliche Veränderungen im menschlichen Selbstverständnis““. 302

Die Primatologie verlängert den Zeitraum in die Tiefe der Evolution hinein, verwendet jedoch ähnliche rhetorische ,traurige Tropen' und hält sich an das elegische Erzählmuster und seine unerfüllbare Sehnsucht nach einem ursprünglichen Zustand. Darin, ein solches ,Goldenes Zeitalter' zu betrauern und des einstigen Glücks in der rückerinnernden Darstellung noch einmal habhaft zu werden, ist die Klage denn auch vollständig elegisch: ${ }^{303}$

[O]nce wild orangutans [...] roamed throughout Asia [...]. $(R E, 396)$

[...] the world of nature as it was before the coming of modern man. [...] African wild life in all its abundance and variety, living as it has always lived [...]. Africa as a land teeming with vast herds of big game $[\ldots] .(Y G, 71)$

In the richness of such a lush environment lived the chimpanzee-like creatures that became the first men. $(T W, 203)$

Die primatologische Klage ist getränkt von dem Bewusstsein, dass dieses Ideal unwiederbringlich der Vergangenheit angehören muss; in dem Sinne gehört sie zu Bohrers „Trauerrede als Reflexionsfigur des je schon Gewesenen: Das präsentische Bewußtsein von dem bevorstehenden Verschwinden eines eben noch gewesenen Augenblicks des Glücks sowie der Erinnerung an schon stattgehabte Abschiede“. ${ }^{304}$ Diese den geschichtsphilosophischen schillerschen Versöhnungsversuch des Sentimentalen untergrabende und insofern subversive, kritische Trauer sieht Bohrer als eben nicht erfolgreiche Trauerarbeit: „Trauer wird nicht

\footnotetext{
${ }^{301}$ Heise: Nach der Natur, S. 48.

${ }^{302}$ Heise: Nach der Natur, S. 76.

${ }^{303}$ Vgl. Bohrer: Historische Trauer und Poetische Trauer, S. 118 f. Wie Burkhard Meyer-Sickendiek aufzeigt, ist die Elegie gerade in Bezug auf die darin verhandelte Emotionsfülle sehr wandelbar. Das Elegische sei mit Schillers Über naive und sentimentalische Dichtung nicht mehr „als melancholische Vergegenwärtigung eines vergangenen, also erlebten Glücks zu begreifen, sondern als Bruch zwischen Natur und Ideal“". Der Elegiker trauert bei Meyer-Sickendiek bzw. Schiller über „die verlorene Natur oder über die Unerreichbarkeit des Ideals, welches aber zugleich elegisch erinnert“ wird. „Wirklich trauern“, so Meyer-Sickendiek, lasse sich „also erst in jenem Moment, in dem sich die Elegie der literarischen Moderne mit Schiller von den antiken Wurzeln löst“. Für ihn ist daher die geschichtsphilosophische Elegie in der Folge Schillers - im Gegensatz zu ihren Vorläufern - als „erste Präfiguration genuiner Trauerarbeit“ zu betrachten. Meyer-Sickendiek: Affektpoetik, S. $122 \mathrm{f}$.

${ }^{304}$ Bohrer: Historische Trauer und Poetische Trauer, S. 120.
} 
aufgehoben, sondern intensiviert." 305 Sie wird also, lässt sich im Sinne der freudschen Trauer/Melancholie-Differenzierung behaupten, zur Melancholie.

\subsubsection{Selfing the Ape}

Der Naturzustand - oder wahlweise das ,Paradies“ -, so sei noch einmal rekapituliert, ist für die primatologische Klage unwiederbringlich verloren, und doch lässt sich davon nicht Abschied nehmen, weil dies das perpetuierte primatische Sterben bedeuten würde. Durch ihre melancholische Warnung ruft die primatologische Klage dazu auf, mit einem gemeinsamen Akt des Handelns zumindest mit einem „remnant“ ( $Y G, 73)$ des Verlorenen, wie Schaller es nennt, die Affen und damit ,uns' zu retten. Darin steckt nicht nur die utopische Vorstellung, ein solcher Akt des Handelns sei auch ein Akt der Stiftung (universeller) Gemeinschaft. Es steckt darin auch ein entscheidender Unterschied zur freudschen Melancholie-Konzeption und auch zu Lévi-Strauss' Rousseau-Lektüre, die das Goldene Zeitalter als Vorstellung einer ,fraternité humaine“306 in ,uns“ allen als verwirklichbar sieht: ${ }^{307}$ Hier wird nicht das verlorene Gegenüber erhalten, in dem es im Selbst eingekapselt wird. Es reicht nicht, uns Menschen als Primaten zu betrachten und Überreste der aussterbenden Affen in uns selbst nachzuweisen. Es wird auch nicht das Ich zum Anderen, an dessen Stelle es schließlich kritisiert werden kann. Stattdessen wird ein Anderes zum Ich und als solches idealisiert: Das verlorene Liebesobjekt wird in die Affen verschoben. In diesen findet man das verlorene Ideal der Einheit (mit) der Schöpfung wieder: „Looking into the calm, unblinking eye of an orangutan we see, as through a series of mirrors, not only the image of our own creation but also a reflection of our own souls and an Eden that once was ours“ (RE, 403), wie Galdikas schreibt. Nur im Überleben der großen Affen, so die Argumentation der primatologischen Klage, überlebt ein Ideal von ,uns“. Statt des , going ape ‘ - der selbst-auflösenden Identifikation mit dem Affen als Anderen - findet hier eine Erweiterung jenes von Lajos Brons wiederum in Rückgriff auf Hegels Dialektik von Identifikation und Verfremdung definierten , sophisticated Othering 'statt. Das ,implicit, and largely unconscious, modeling of the other as self by assuming that what's true for the self is true for the encountered other as well“'308 wird zum , selfing the ape': zu einer melancholischen Vereinnahmung des Affen im Dienste der Rekonstitution eines verlorenen Ich-Ideals.

\footnotetext{
${ }^{305}$ Bohrer: Historische Trauer und Poetische Trauer, S. 120. Bohrer sieht Schillers „Trauer um den Verlust einer zivilisatorischen Epochenidee und deren Rettung“ von Goethes „Trauer um einen Augenblick des Subjekts, der nicht mehr rettbar ist" abgelöst; man könne auch sagen, dass damit eine unauflösbare Melancholie in die Elegie einkehrt. Bohrer: Der Abschied, S. V.

${ }^{306}$ Lévi-Strauss: Tristes Tropiques, S. 421.

${ }^{307}$ „Rien n'est joué; nous pouvons tout reprende. Cequi fut fait et manqué peut être refait: ,L'âge d'or qu'une aveugle superstition avait placé derrière [ou devant] nous, est en nous ". Lévi-Strauss zitiert hier die Devise des ,journal saint-simonien Le Producteur“. Lévi-Strauss: Tristes Tropiques, S. 421, 1770 f., Hervorheb. im Original.

${ }^{308}$ Brons, Lajos: Othering, an Analysis. In: Transcience 6/1 (2015), S. 69-90, hier S. 71.
} 


\subsection{Jammer und Schaudern: Fiktion als kathartischer Raum für Forschungsemotionalität}

\subsubsection{Rekapitulation: Affektive (Ver-)Formungen}

Ob melodramatischer Modus, Fallgeschichte, Zeugen- oder Kriegsbericht, Nachruf oder primatologische Klage - die Form, welche Primatolog/innen in ihren Forschungsmemoiren wählen, um von herausfordernden Beobachtungen und Erlebnissen zu erzählen, steht im Zusammenhang mit deren affektivem, epistemologischem, evaluativem und - wie zuletzt an den ,traurigen Tropen' gezeigt - auch politischem Potenzial. Denn die Erzählung, so Albrecht Koschorke,

verknüpft Ereignisse und Aktionen zu Episoden, den Untereinheiten der Erzählsequenz, und lässt durch deren Kombination Handlungsmuster entstehen, die ihrerseits auf die Weltorientierung und das Selbstverständnis der Akteure zurückwirken. Zu den kognitiven treten emotive und evaluative Funktionen, denn immer spielen sowohl Affektbesetzungen als auch die Verständigung über Werte in die erzählten Vorgänge hinein. ${ }^{309}$

Unterschiedliche Formen des Erzählens, im Vorangegangenen als Genres und Textformen bezeichnet, korrespondieren mit unterschiedlichen Erfahrungssituationen und liefern Muster für eine solche narrative und darin eben vor allem auch affektive und epistemische Bearbeitung, Anordnung, Darstellung und Vermittlung dieser Erfahrungen. Koschorke bezeichnet Erzählen aus diesem Grund auch als Problemlösungsstrategie und Komplexitätsreduzierung: „Das Erzählen dient also der Bearbeitung und Auflösung einer , mich betreffenden Sorge “".310 Die vorangegangenen Kapitel haben anhand des Sexualverhaltens, der Gewalt und des Todes bzw. des Sterbens erörtert, wie die Autor/innen ihre ethologischen ,Sorgen ‘ durch die verschiedenen Formen in ihren primatologischen Forschungsmemoiren bearbeiten - und in diesen konventionalisierten Genres aufzulösen versuchen.

Im spezifischen Fall der herausfordernden Beobachtungen der Langzeitforschung mit Affen entstehen durch die Genre-Auswahl der Primatolog/innen, wie etwa am melodramatischen Modus oder den Normalisierungs- bzw. Pathologisierungsstrategien von Kriegsbericht vs. Fallgeschichte gezeigt wurde, zudem unterschiedliche epistemologische Rückkopplungen, indem verschiedene Formen der Erzählung ihren Gegenstand unterschiedlich affektiv besetzen und evaluativ verhandeln. Daraus kann nicht nur im Rahmen wissenschaftlicher Paradigmen eine ontologische Normalisierung zunächst affektlogisch problematischen Verhaltens erfolgen, wie bspw. beim Vernichtungskrieg unter den Gombe-Schimpansen oder angesichts des problematischen Sexualverhalten der Orangutans. Es kann, wie man am Beispiel des primatologischen Nachrufs und der melancholischen Klage sehen kann, auch ein (gesellschafts-)politisches und ethisches Potenzial des Genre-Einsatzes erwachsen.

\footnotetext{
${ }^{309}$ Koschorke: Wahrheit und Erfindung, S. 20.

${ }^{310}$ Koschorke: Wahrheit und Erfindung, S. 69.
} 


\subsubsection{Formen des Erzählens: William Boyds Brazzaville Beach (1990)}

Dieser Zusammenhang von Erfahrung, Erzählung und Genre sowie der Intention des Berichtens, macht wiederum die Primatologie für fiktionale Erzählungen interessant: „Another problem: how do I begin? How do I tell you what happened to me? [...] Which voice do I use? I was different then; and I am different now. “311 Mit diesen Worten im Prolog seines Romans Brazzaville Beach lässt William Boyd die Protagonistin und - teils - Erzählerin Hope ein Problem benennen, das auch in den vorangegangenen Abschnitten erörtert wurde: Wie lässt sich von den Beobachtungen und Erfahrungen adäquat erzählen, die Hope zu dem Punkt in der Gegenwart gebracht haben, von dem aus sie erläutern möchte, warum sie ist, wo und wie sie ist?

Die Biologin Hope entschließt sich, ihre Erzählung autodiegetisch ${ }^{312}$ mit einer ethologischen Beobachtungsszene im mosambikanischen Wald beginnen zu lassen. Als Affenforscherin an der Grosso Avore Research Site hat sie Kannibalismus und einen Vernichtungskrieg unter Schimpansen beobachtet. Um zu berichten, ,what happened to me“", muss Hope jedoch weiter ausholen. So wird uns auch im Modus der internen Fokalisierung auf die Protagonistin durch eine heterodiegetische Erzählinstanz von Hopes davor liegender Ehe mit dem Mathematiker John Clearwater erzählt. Reflektierende, in der Perspektive zwischen homodiegetischer und heterodiegetischer Erzählposition wechselnde, aber weiterhin intern fokalisierte, kursiv gesetzte Passagen diskutieren dazwischen meist (aber nicht ausschließlich) mathematische Konzepte in ihrer Anwendbarkeit oder Übertragbarkeit auf Elemente der ,histoire‘. ${ }^{313}$ Und immer wieder berichtet die Erzählerin Hope autodiegetisch auch aus der Gegenwart des Erzählens, die sie in einem Strandhaus am titelgebenden Brazzaville Beach, ,on the edge of Africa“ (xi), verbringt.

Die neuen Erkenntnisse der primatologische Feldforschung der 1970er Jahre nehmen in Brazzaville Beach eine prominente Stellung ein. Die Übereinstimmung in der Schilderung des Kannibalismus und der tödlichen Gewalt unter den Schimpansen im Roman mit den Passagen aus Jane Goodalls Through a Window und die explizite Kenntnis von Wissensbeständen und Personal der Schimpansenforschung verweist auf die fundierte Beschäftigung Boyds mit dem ,Feld' primatologischen Wissens und Forschens sowie mit der affektiven Valenz der Feldforschung und ihren epistemologischen Umwälzungen. ${ }^{314}$ Als Zeugin verzeichnet die Figur

\footnotetext{
${ }^{311}$ Boyd, William: Brazzaville Beach. London 1990, S. xiii. Im Folgenden, wenn zur Unterscheidung nötig, als $B B$ geführt; Seitenangaben im Text.

${ }^{312}$ Vgl. Genette, Gérard: Die Erzählung. München 1994, S. 176 ff.

${ }^{313} \mathrm{Vgl}$. Genette: Die Erzählung, S. 16 f.; $132 \mathrm{ff}$.

${ }^{314}$ Auch bei Boyd entwendet ein Mutter-Tochter-Gespann (Rita-Lu und Rita-Mae) einer Schimpansin ihr Baby und beißt ihm in den Kopf. Vgl. BB, S. 98 f. Die im Roman geschilderten tödlichen Angriffe der Schimpansen aus dem Norden gegen die durch Abwanderung entstandene südliche Gruppe ruft jene traumatischen Details der Gewalt auf, die Goodall bis in ihre späteren Schriften verfolgen. Vgl. z. B. BB, S. 135 f. und Abschn. 3.3.4 der vorliegenden Studie.
} 
und Erzählerin Hope nicht nur den Schrecken über die beiden Formen von intraspezifischer Aggression, sondern auch die Schwierigkeit, ihre Beobachtungen und Schlüsse zu teilen und ihre Kolleg/innen und vor allem den Leiter des Forschungscamps davon zu überzeugen, dass Schimpansen keine inhärent friedliche Spezies sind, sondern sich bereitwillig gegenseitig töten. Diese Erkenntnis wird anders als bei Goodall, die sich in ihren Verschriftlichungen der Vorkommnisse damit zu trösten versucht, dass sich die Schimpansen ihres Tuns nicht bewusst sein können, kritischer ausgewertet: In einer reflektierenden, kursiven Passage, die auf die erste der tödlichen Attacken folgt und nach dem Schimpansen, der Mr Jebs Bein so lange verdreht, bis es bricht, mit „PULUL“ betitelt ist, zitiert Hope scheinbar aus einem Wörterbuch: „Cruelty. (Kru.elti). n. (1) The quality of being cruel; disposition to inflict suffering; delight in, or indifference to, another's pain; mercilessness, hard-heartedness“ (139). Wie in den anderen dieser reflektierenden Passagen wird anschließend ein Konzept - jenes der Grausamkeit - hinsichtlich seiner begrifflichen Anwendbarkeit exploriert:

\footnotetext{
When Hope thinks of Mr Jeb's slow death, she remembers most vividly the way Pulul sat on the old chimp's back, twisted his leg until it broke, and then tried to bite his toes off. It was a cruel act; it looked cruel. But did he know what he was doing? ,delight in, or indifference to, another's pain. 'If it was cruel, then it was deliberately done. If it was deliberately done, then blind instinct has to be ruled out, some level of cognitive awareness must be involved.

Hope knows (how do you know?) that this was the evil in the chimpanzee. Pulul wanted to inflict pain, as much as possible. (139)
}

Mit dieser Passage verweigert sich die Erzählerin Hope der goodallschen Versöhnung mit dem Schrecklichen und fragt gleichzeitig nach dem Entstehen und der Beschaffenheit von Wissen und Erkenntnis: How do you know?

Diese Frage und die prominente Stellung der Primatologie im Roman, ebenso wie die Vielfalt an Erzählperspektiven und -ebenen haben Julika Griem dazu veranlasst, für diesen Text im Rahmen ihrer Studie zu Affen als Figuren anthropologischer und ästhetischer Reflexion primär unterschiedliche Problematisierungsvermögen geltend $\mathrm{zu}$ machen. In seinen ,primatologischen Abschnitten, in denen die Szenen des Kannibalismus und Totschlags aus Jane Goodalls Memoiren nacherzählt werden, geht es für Griem um „das Problem der hermeneutischen Bewältigung kontingenter Verhaltensweisen bei Primaten", 315 wie es auch in den vorangegangenen Kapiteln an der Erzählform in den Forschungsmemoiren untersucht wurde. In der erzählformalen Vielfalt des Romans, die mit ihren aus der Mathematik entliehenen Begriffen, Konzepten und Modellen explizit die „Analogie als Erkenntnis- und Darstellungsmodus“316 verhandle, aber auch weiter gefasst in Bezug auf die Narrative der anthropomorphisierenden Primaten-Forschung, betreibe der Roman laut Griem eine

\footnotetext{
${ }^{315}$ Griem: Monkey Business, S. 244.

${ }^{316}$ Griem: Monkey Business, S. 245.
} 
„,intradiegetische Problematisierung von Analogiebildungen“317 und damit eine „Problematisierung des Zusammenhangs von Beobachtung und Spekulation“.318 Der Perspektivenwechsel von Passage zu Passage ermögliche es zudem der Erzählerin, eine objektivierende Distanz zu sich selbst herzustellen, sich damit als Primatologin selbst zum Forschungsobjekt zu machen und $\mathrm{zu}$ beobachten, darin Selbsterkenntnis und in deren Verlängerung Erkenntnis über die Primatologie zu gewinnen. ${ }^{319}$ Griem spitzt diese Thesen zu einem Leitmotiv ihrer knappen Interpretation des Romans zu: Durch diese Ebenen der Problematisierung betone Boyds Roman die „Kontingenz und Inkompatibilität sowohl menschlicher als auch tierischer Kommunikationsversuche“.320

Griems schlüssige Interpretation des Romans als Problematisierungsmedium für primatologische Forschung und Erkenntnis soll im Folgenden um einen vernachlässigten Analyseansatz ergänzt werden. ${ }^{321}$ Zwar erwähnt Griem knapp eine Rolle vom Emotionalität zumindest auf der Darstellungsebene des Schreibens über Affen, wenn sie Boyd konstatiert, durch Distanznahme einer Schilderung der Schimpansen in ,moralisierender oder sentimentaler Weise“"322 ausgewichen zu sein, wie sie z. B. in Michael Crichtons Congo Anwendung finde. Ihre Konzentration auf die Kommunikationsschwierigkeiten und die Problematisierung von Analogieschlüssen in der Wissensgenerierung der Forschung mit Affen scheint mir jedoch William Boyds emphatischen Fokus auf Emotionalität in Brazzaville Beach auszublenden.

„I was different then; and I am different now“: Die von Boyds Erzählerin Hope formulierte Schwierigkeit, sich auf eine adäquate Erzählform für das Geschehene festzulegen, basiert in diesem Roman auf der Einsicht, dass die zu erzählenden Ereignisse die Erzählerin fundamental verändert haben. Die Hope, welche dem Mathematiker John Clearwater mit einer Bestimmtheit nachstellt, die sie sich kaum noch erklären kann (,she could not say why her instinct was so empathic, but she was sure that this was her man“, 14), ist eine andere als

\footnotetext{
${ }^{317}$ Griem: Monkey Business, S. 245.

${ }^{318}$ Griem: Monkey Business, S. 250.

${ }^{319}$ Griem: Monkey Business, S. 247.

${ }^{320}$ Griem: Monkey Business, S. 252.

${ }^{321}$ Griems Analyse fällt knapp aus, da sie sich für ein vor dem Hintergrund des Aufbaus ihrer Studie nachvollziehbares aber $\mathrm{m}$. E. für die Lektüre des Romans auch einengendes komparatistisches Verfahren entscheidet und Brazzaville Beach alleinig im Vergleich mit Michael Crichtons Congo liest. Griem sieht zudem die Problematik scheiternder menschlicher und tierlicher Kommunikationsversuche als ausschlaggebend für Boyds Porträt der Primatologie. Diese Problematik sei symbolisch in einer Fabel verdeutlicht, die Hope von einem ihrer mosambikanischen Feldassistenten erzählt bekommt. Diese Fabel, so lässt sich einwenden, zielt weniger auf Kommunikationsschwierigkeiten ab als auf das in dieser Arbeit bereits angeführte, von Thomas Nagel erörterte Problem von der epistemologischen Unzugänglichkeit des anderen (tierlichen) Bewusstseins. Nagel: What It Is Like to Be a Bat? In Brazzaville Beach wird dieses erkenntnistheoretische Problem ironisch weitergedacht als die Unzugänglichkeit jedes anderen als des eigenen Bewusstseins: „,You are not me', Ntiono said [to Iko]. ,How do you know that I do not know the happiness of the chimpanzee “ " $(B B, \mathrm{~S} .125)$.

${ }^{322}$ Griem: Monkey Business, S. 249.
} 
die selbstbewusste Botanikerin Hope, die eine Heckendatierungsstudie in Nordengland vornimmt (,Her mind was calm and full of her task and all her senses were stimulated as she crouched at the foot of a hawthorn hedge in a landscape she had come to know as intimately as any in her life", 106). Sie ist auch eine andere als die furchtlose Ethologin Hope, die von mosambikanischen Rebellen gefangen genommen wird (,My shoulder was still sore, but I was not frightened. I was tense, certainly not at ease, but these lanky boys with their rationed guns and the diminutive Dr Amilcar did not frighten me“, 221), und eine andere als die Erzählerin Hope, die scheinbar affektlos aus der Gegenwart des Strandes heraus ihre Geschichte(n) erzählt. Boyds Griff zu unterschiedlichen Erzählperspektiven und Zeitebenen korrespondiert, so lässt sich wiederum zugespitzt feststellen, mit der in den vergangenen Kapiteln thematisierten Suche der Forschungsmemoiren nach der adäquaten Textform für affektiv hochvalente Beobachtungen und Phänomene. Aber Boyds Roman geht darüber noch hinaus und setzt einen Fokus auf das Wesen, die Bedeutung und die richtige Form von Emotionalität.

\subsubsection{Kathartische Implikationen}

Etwa mittig in Boyds Brazzaville Beach findet sich die Schilderung eines bemerkenswert intensiven Gefühlsausbruchs. Zu diesem Zeitpunkt ist im Roman durch Hopes Bericht etabliert, dass John Clearwaters „eccentricities were becoming problems, and that quirks of behaviour were becoming warning signs" (124). Später werden diese Exzentrizitäten und Verhaltensauffälligkeiten zur Diagnose einer manisch-depressiven Erkrankung beitragen (216 f.). John Clearwaters Stimmungsschwankungen kulminieren an dieser Stelle in einem Gefühlsausbruch, dessen Auslöser Johns Erläuterung eines auffälligen und für seine Umgebung disruptiven Verhaltens ist. Es handelt sich um eine manuelle Tätigkeit, welche ihm als Erkenntnisinstrument dienen sollte und eine Reflexion über verschiedene Denkweisen hervorgerufen hat: ${ }^{323}$

She [Hope] saw that his eyes were brimful of tears. [...] He made a strange sound - half grunt, half retch - in the back of his throat. [...] His entire body seemed to give a shudder and his face went bright red. He closed his eyes and pressed his chin to his chest. For a moment, Hope thought he was going to vomit. But he wept. He put his hands on the table, hunched forward and let the sobs blurt from him. He made a strange, panting, wailing noise, his mouth hanging open, tears, snot and saliva dripping from his face. [...] She was terrified by the sound he was making, as if it were a prototype form of weeping, unfamiliar and unrecognizable. ,Johnny, darling, stop! Please!

\footnotetext{
${ }^{323}$ Bei einem Besuch Johns an Hopes Arbeitsort in Knap, wo sie auf einem Gelände Hecken datiert, beginnt John tiefe, das Gelände beschädigende Löcher zu graben. Der Gefühlsausbruch schließt sich direkt an die Reflektion dieser Grabe- als Denktätigkeit und ihrer Flüchtigkeit an „it's going again, getting weak [...]. I had the gift for a few months. On loan“ (BB, S. 142). John realisiert im Restaurant mit Hope, „how terrible it is, to have something, that kind of power, and then have it taken away from you" " $B B$, S. 143).
} 
The whole restaurant had fallen immediately silent, disturbed and unsettled. Beneath her hands, Hope could feel the muscles of his shoulders locked and quivering. He was letting his sadness run from him, she thought. She could almost sense it coiling about her - a thin and ethereal flux - like a gas, a turbulent gas, flowing from his mouth, nose and eyes.

[...] As she led John through the restaurant, she was aware of the rapt and troubled faces of the other diners staring at them both. What was this abject misery, they seemed to be demanding? This was a man: why was he so afflicted? What shocking tragedy had reduced him so? To her shame, Hope felt a hot embarrassment envelop her like a shawl. (143, Hervorheb. im Original)

Diese Passage des Romans ist zentral für Boyds/Hopes Verständnis von Emotionalität: Erstens bricht der Affekt hier anhand eines forschungsrelevanten Denk- und Erkenntnisproblems aus. Zweitens wird dieser Ausbruch mit genauem Blick für somatische Details der affektiven Reaktion beschrieben: das Weinen, das in seiner stärksten Form an das Erbrechen erinnert, an dem der ganze Körper konvulsiv beteiligt ist, und das mit einem Geräuschspektrum von Grunzen, Ausstoßen, Keuchen und Heulen (,grunt“, „,blurt“, „panting“, „,wailing“) dem Weinenden das menschliche Antlitz zu nehmen scheint. Was John hier produziert, wirkt als ,prototype form of weeping“, also noch nicht ausgeformt, zivilisiert und lesbar, sondern: „unfamiliar and unrecognizable“, „disturb[ing] and unsettl[ing]“. Es löst weniger Mitgefühl als Schrecken aus (,She was terrified by the sound“). Drittens wird diese überwältigende, durch ihre Urform entstellende Emotion hier in Bildern manifest; manifest in jenem Sinn, in dem es sich nicht mehr allein um psychische Affektphänomene handelt, sondern um Analogien fassbarer oder fühlbarer - Entitäten im Raum: Johns Traurigkeit (,sadness“) wirkt auf Hope wie ,,a thin and ethereal flux“, ,a turbulent gas, flowing from his mouth, nose, and eyes“, schlimmer noch, ,she could almost sense it coiling about her". Vergleichbar fühlt sich Hopes Beschämung (,embarrassment“) nicht nur bekannt-somatisch ,heiß“ an, sondern „envelop[ed] her like a shawl“. Viertens werden anhand der Reaktionen von Hope und den anderen Restaurant-Gästen die sozialen Grenzen des Verständnisses für derart fundamentale, essenzielle Gefühlsäußerungen aufgezeigt. Diese unvermittelte Form des Affekts ist so erschreckend wie faszinierend und verstörend; sie ruft „rapt and troubled faces“ hervor. Gerade darin zeigt sich, wie nah Boyds Konzeptualisierung von Gefühl in Brazzaville Beach an einem aristotelischen Emotionsbegriff orientiert scheint: „This was a man; why was he so afflicted?“. Die Antwort kann bei ,abject misery“ und „,shocking tragedy“ gesucht werden, bei einer kathartischen Aufführung von Emotionalität und der Verhandlung ihres ,rechten Maßes'.324

\footnotetext{
${ }^{324}$ Mit dem ,rechten Maß` soll hier Aristoteles’ in der Nikomachischen Ethik anhand des modellbildenden Zorns verhandelte Mesotes-Lehre, d. h. die Frage nach der tugendhaften Haltung in Bezug auf Affektausprägungen, aufgegriffen werden. In Buch IV heißt es dort: „Dies ist immerhin klar, daß die Mitte lobenswert ist [...]. Übermaß und Mangel dagegen sind verwerflich, in kleinem Umfang wenig, in größerem mehr, in ganz großem außerordentlich. Man muß also offensichtlich die mittlere Haltung einnehmen“. Aristoteles: Die Nikomachische Ethik: griechisch/deutsch. Neu Hg. von Rainer Nickel. Düsseldorf/Zürich 2001, S. 173.
} 
In ihrer Analyse der Ugly Feelings spricht Sianne Ngai davon, die von ihr in der Literatur untersuchten Gefühle seien ,,noncathartic feelings“",325 die ,give rise to a noncathartic aesthetic: art that produces and foregrounds a failure of emotional release (another form of suspended ,action") and does so as a kind of politics". ${ }^{326}$ Diese These wird durch das Gefühl der Irritation illustriert, wie es sich unauf- und unerlösbar durch den diesbezüglich von Ngai betrachteten Roman ${ }^{327}$ zieht und hier bereits erörtert wurde (vgl. Abschn. 3.1.1). Obwohl der Begriff ,Katharsis wegen seiner genitivbedingt so vielfältigen wie unterschiedlichen Auslegungen ein schwieriger ist, lässt sich doch an Brazzaville Beach beobachten, dass William Boyd nicht nur einen ,viszeralen ${ }^{6328}$ Emotionsbegriff ausbildet und den epistemologischen Prozess der Ver-Emotionalisierung des Affekts konzeptualisiert, sondern auch im Gegensatz zu der von Ngai untersuchten Literatur Fiktion gerade als kathartischen Raum von Emotionalität für die Leserschaft anbietet. ${ }^{329}$ Führt die Irritation in jener von Griem identifizierten hermeneutischen Bewältigung kontingenter Verhaltensweisen bei Primaten in den Forschungsmemoiren zu einer affektiv konnotierbaren Formen- und Genrevielfalt, so scheint Boyd durch die erzählerische Vielfalt eine affektive Bewältigung jener Verhaltensweisen nicht nur zur Aufführung zu bringen. Vor dem Hintergrund von Boyds viszeralem Verständnis von Gefühlen und seiner Nähe zur postmodernen Emotionsphilosophie, die zum Teil psychoanalytisch informiert in der Versprachlichung von Affekten einen

\footnotetext{
${ }^{325}$ Ngai: Ugly Feelings, S. 9.

${ }^{326}$ Ngai: Ugly Feelings.

${ }^{327}$ Larsens, Nella: Quicksand. New York/London 1928; vgl. Ngai: Ugly Feelings, S. 174 ff.

${ }^{328}$ Mit der Verwendung des Begriffs des ,Viszeralen“ knüpfe ich an ein neueres neurowissenschaftliches Emotionsverständnis an, welches eine komplexe Interaktion zwischen kognitiven und sozialen Prozessen und physiologischem Feedback annimmt, aber physiologische Maße als Indikatoren für emotionale Prozesse betrachtet. Damit grenzt es sich ab von der James-LangSchule, die physiologische Veränderungen als Auslöser emotionaler Prozesse betrachtet. Vgl. auch Feldman Barrett, Lisa: Emotions Are Real. In: Emotion 12/3 (2012), S. 413-429.

${ }^{329}$ Die Problematik der ,richtigen ‘ Deutung des Katharsis-Begriffs wird von Bernd Seidensticker anhand vier grundsätzlich verschiedener, um die Mehrdeutigkeit des griechischen Genitivs der aristotelischen Formulierung kreisender Erklärungen der Katharsis verhandelt: 1. Katharsis als emotionale purificatio, d. h. als quantitative oder qualitative Reinigung tragischer Emotionen; 2. Katharsis als purgatio, d. h. als Abfuhr bzw. reinigende und erleichternde Befreiung von Emotionen; 3. Katharsis als clarificatio, d. h. ,als intellektuelle Klärung der tragischen Ereignisse, die der Zuschauer im Durchgang durch die Tragödie als signifikant und allgemeingültig für die menschliche Existenz zu begreifen lernt"; und 4. Katharsis als intellektuelle purificatio, „als Reinigung der tragischen Ereignisse durch die Demonstration, daß der Held unschuldig“, sein Handeln also nicht abscheulich sei. Obwohl Seidensticker die Entscheidung zwischen den beiden Versionen, die Katharsis als emotionale Erfahrung verstehen (1. und 2.), als schwierig bezeichnet, befürwortet er eine medizinisch-therapeutische Deutung des aristotelischen Katharsis-Begriffs. Vgl. Seidensticker, Bernd: Die Grenzen der Katharsis. In: Martin Vöhler/ Dirck Linck (Hg.): Grenzen der Katharsis in den modernen Künsten. Transformationen des aristotelischen Modells seit Bernays, Nietzsche und Freud. Berlin/New York 2009, S. 3-20, hier S. $15 \mathrm{ff}$.
} 
kathartischen Prozess sieht, der Energie (Brennan) oder Intensität (Massumi) freisetzt, lässt sich sogar an die medizinisch-pathologische Katharsis-Vorstellung von Jacob Bernays und in Folge der Psychotherapeutik anknüpfen. ${ }^{330}$

Um es jedoch gleich vorweg zu nehmen: Brazzaville Beach ist kein Tragödienstoff. Es geht aber sehr deutlich bei Boyd - anhand des Mathematikers John Clearwater und des Primatologen Eugene Mallabar - um übermäßige, nicht adäquate, oder pathologische Emotionalität, wie sie Jacob Bernays' Katharsis-Interpretation zugrunde liegt. Bernays rückt durch den Bezug zur aristotelischen Politik die Katharsis nicht unter einem moralischen oder hedonistischen, sondern unter einem ausgewiesen ,pathologischen Gesichtspunkt ${ }^{\star 331}$ in den Blick. Katharsis ist ihm

eine von Köperlichem auf Gemüthliches übertragene Bezeichnung für solche Behandlung eines Beklommenen, welches das ihn beklemmende Element nicht zu verwandeln oder zurückzudrängen sucht, sondern es aufregen, hervortreiben und dadurch Erleichterung des Beklommenen bewirken will. ${ }^{332}$

Durch die „Solliciation des störenden Stoffes“, in der Tragödie also das ,,zur Aeusserung [H]ervordrängen“ der beklemmenden, aufgestauten Emotionen, soll „das verlorene Gleichgewicht wiedergewonnen“"333 werden. Die Psychoanalyse greift dieses Modell in der ,kathartischen Analyse“ auf, die zunächst statt des Vorführens des Theatermodells ein ,Wegerzählen“ im Narrativ verfolgt. ${ }^{334}$ Boyds Fokus auf

\footnotetext{
${ }^{330}$ Siehe Brennan, Teresa: The Transmission of Affect. Ithaca/London 2004; Massumi, Brian: The Autonomy of Affect. In: Cultural Critique 31 (1995), S. 83-109; Bernays, Jacob: Grundzüge der verlorenen Abhandlung des Aristoteles über Wirkung der Tragödie. Breslau 1857.

${ }^{331}$ Bernays: Grundzüge, S. 141. Linck/Vöhler weisen darauf hin, dass bereits die rein/unreinOpposition bei Aristoteles eine derartige ,pathologische“ Deutung nahelege: „Die Zuschauer der Tragödie durchlaufen den Prozeß einer emotionalen Anspannung, der angesichts der folgenden Katharsis als eine Phase der Verunreinigung zu verstehen ist, auf die eine gegenläufige Phase der Entspannung und Befreiung folgt. Zum Gegenstand des Reinigungsvorgangs werden die im Zuschauer erregten (nach der Logik der eingesetzten Metaphorik: verunreinigten) Gefühle“. Linck, Dirck/Vöhler, Martin: Zur Einführung. In: Dies. (Hg.): Grenzen der Katharsis in den modernen Künsten. Transformationen des aristotelischen Modells seit Bernays, Nietzsche und Freud. Berlin/New York 2009, S. IX-XIV, hier S. X.

${ }^{332}$ Bernays: Grundzüge, S. 144.

${ }^{333}$ Bernays: Grundzüge, S. 176.

${ }^{334}$ Gödde verweist darauf, wie Joseph Breuer in seinem Kreuzlinger Krankenbericht im Fall von Anna O. (Bertha Pappenheim) als Heilfaktor das „Wegerzählen“ bzw. „die Sache heruntersprechen" benennt; Gödde, Günter: Therapeutik und Ästhetik - Verbindungen zwischen Breuers und Freuds kathartischer Therapie und der Katharsis-Konzeption von Jacob Bernays. In: Martin Vöhler/Dirck Linck (Hg.): Grenzen der Katharsis in den modernen Künsten. Transformationen des aristotelischen Modells seit Bernays, Nietzsche und Freud. Berlin/New York 2009, S. 63-92, hier S. 65 f. Gödde zitiert aus Breuer/Freud: ,in der Sprache findet der Mensch ein Surrogat für die Tat, mit dessen Hilfe der Affekt nahezu ebenso ,abreagiert “ werden kann“ (S. 68, Hervorheb. im Original), und nennt diese ,Psychokatharsis“ eine „emotional korrigierende Erfahrung“ (S. 73). Vgl. Breuer, Josef/Freud, Sigmund: Über den psychischen Mechanismus hysterischer Phänomene. Vorläufige Mitteilung [1893]. In: Freud, Sigmund: Gesammelte Werke. Bd. 1. Hg. von Anna Freud u. a. Frankfurt a. M. 1999, S. 81-89.
} 
Emotionalität im Forschungskontext und ihre ausführliche somatische Darstellung im Fall einer katastrophischen Explosion dort, wo sie vorab nicht ,rechtmäßig ' ausgeübt werden konnte, lässt sich als solche Artikulation des Beklemmenden deuten, als ,Wegerzählung' der problematischen ,pathologischen' Forschendenemotionalität und einer Verhandlung der ,guten' epistemologisch nutzbaren Emotionalität im ,rechten $\mathrm{Ma}^{3}$ ‘. Dafür erstellt Boyd zunächst einen Konnex von Forschung und Emotionalität. Anhand des Hauptpersonals des Romans werden mit analytischem Blick auf das Erleben und Beschreiben von Emotionen dieselben als viszerale und epistemische Phänomene begriffen. Unter Zuhilfenahme von aus den Naturwissenschaften entliehenen Modellen konzeptualisiert der Roman zudem Emotionalität in einem Sinn, der mit einer von Arbogast Schmitt herausgearbeiteten rationalen Kultur des Gefühls bei Aristoteles ${ }^{335}$ korrespondiert und Ideen neumaterialistischer Emotionstheorien der Postmoderne aufgreift. Zentral scheint dabei die Frage danach, wie Emotionalität im rechten Maß als Erkenntnismedium dienen kann.

\subsection{4 "systems in flux ${ }^{\prime 336}$ : Emotionalität und Forschung}

Neben John, bei dem explizit in der psychischen Erkrankung Emotionalität und Forschung zusammengedacht werden, nutzt Boyd zwei weitere Figuren, um Emotionalität anschaulich werden zu lassen: Zentral ist die Protagonistin Hope, bei der durch ihre privilegierte Position als Erzählerin und die verschiedenen Erzählebenen Forschungs- und ,private' Gefühle zugleich betrachtet werden können. Aber auch Hopes Vorgesetzter, der etablierte Schimpansenforscher Eugene Mallabar, dient als Affektträger. An allen drei Figuren wirken epistemische Prozesse affektiv. Dabei stellt der Roman die beiden Männer und Hope einander dadurch gegenüber, wie sie den Bereich ihrer Emotionalität beherrschen oder nicht beherrschen können. Eine Buchbesprechung aus dem Jahr 1991 setzte den Fokus des „superior suspense yarn“ denn auch auf die Frage: „Will our heroine, who is no crusader but merely following scientific principles, prevail against the murderous plots of an evil genius defending his golden poppycock eggs?““.337 Die beiden männlichen Figuren erscheinen als ,,scientists who become so obsessed with their theories that they lose their grip on real life“, 338 Hope dagegen ist eine „capable, active, admirably pragmatic person “. ${ }^{339}$ Die Literaturkritik bringt damit nicht nur eine Figurenkonstellation auf den Punkt, sondern hebt auch Boyds Schachzug hervor, eine exzessive bis pathologische Forschendenemotionalität in den männlichen Figuren zu verorten und die weibliche Protagonistin Hope entgegen der Figuration

\footnotetext{
${ }^{335} \mathrm{Vgl}$. Schmitt: Kommentar.

${ }^{336} \mathrm{BB}$, S. 45.

${ }^{337}$ Duffy, Martha: Monkeys in a Jungle - Brazzaville Beach by William Boyd. In: Time 137/25 (1991), S. 64.

${ }^{338}$ Duffy: Monkeys in a Jungle, S. 64.

${ }^{339}$ Duffy: Monkeys in a Jungle, S. 64.
} 
der Primatologin als ,crusader" und ,ape-hugger' als Beispiel einer ,vernünftigen ‘ Emotionalität zu gestalten. Doch ganz so einfach oder schematisch - hier die obsessiv von ihrer Forschung affizierten Männer, dort die pragmatische, nüchterne Frau - geht Boyd natürlich nicht vor, wie das Folgende zeigen wird.

Eugene Mallabar ist bei Weitem kein ,evil genius', sondern eine männliche Version Jane Goodalls: „,A] handsome man“ (9) mit einer „dauntingly positive air“ (9), ,he was quite good company and it was fascinatingly instructive to observe the chimpanzees with him“ (202). Mallabar hat die Forschungsstation Grosso Avore aufgebaut und das Buch zu den Schimpansen geschrieben. Ein weiteres soll in der Erzählebene, auf der Hope die Schimpansengewalt beobachtet, unmittelbar folgen. Hope gesteht bereitwillig ein: „I saw that he had an understanding of these apes that was profound and, there was no other word for it, full of love" (202, Hervorheb. MS). Doch Mallabars Vision der Schimpansen und sein affektives Verhältnis sowohl zur Art als auch zu seinen eigenen Forschungsbemühungen beeinflussen seine Wahrnehmung bzw. seine Erkenntnisfähigkeit. So muss auch die Reaktion auf neue Erkenntnisse, wenn sie schließlich durch die Schutzschicht seines Dogmas von der friedliebenden Spezies durchbrechen, problematisch ausfallen: Als Hope ein totes, angefressenes Schimpansenbaby zur Obduktion ins Camp bringt, deutet Mallabar es schlicht zum Pavian um und lässt die Leiche ebenso wie Hopes Aufzeichnungen verschwinden (26 ff., 86 ff.). Auf Hopes Hinweise und Anmerkungen zur Schimpansengewalt reagiert er herablassend (,Observe and note. Observe and note. Leave the interpretation to me“, 184) und mit dem Vorwurf der Unprofessionalität: „These ... these allegations you've made are pure speculation. You are jumping to conclusions based on the patchiest data. Bad. Bad science, Hope. Whatever you may think is happening is wrong. You are wrong, Hope“ (184, Hervorheb. im Original). Doch die vermeintliche fachliche Gewissheit und das Überlegenheitsbewusstsein sowie sein Vertrauen in das von ihm geformte Bild der friedlichen Schimpansen lassen sich schließlich nicht aufrechterhalten. Hope manipuliert ihn dahingehend, sie einige Tage im Feld zu begleiten, damit er das Morden unter den Schimpansen mit eigenen Augen an- und somit endlich einsehen kann. Als er einen tödlichen Angriff unter den Schimpansen miterlebt, bricht für Mallabar sichtbar eine Welt zusammen:

The fight had only lasted a few minutes. I felt myself begin to unfreeze. I looked around at Mallabar. His face was sallow, bloodless; his beard looked suddenly black and coarse. He was biting his bottom lip, staring in front of him as if he were in terrible shock. I touched his shoulder; I could feel it shuddering beneath my fingers.

,Jesus', he said. ,Jesus Christ.' He kept on repeating this.

[...] He stared, clearly horrified, at Lester's body. [...] I felt enormously sorry for the man. (205 f.) 
Eugene Mallabars Reaktion fällt jedoch, sobald er erfährt, dass dies nicht das erste Vorkommnis ist, vom Schock in die Aggression. Er macht Hope für das Geschehen verantwortlich und greift sie tätlich an:

He raised his fist to shoulder level, arm bent and took a step towards me. [...] ,I blame myself, ' he said. He looked up. ,I should have had you supervised. 'Then he screamed at me, madly: ,WHAT HAVE YOU DONE? WHAT HAVE YOU DONE?'

I took a step back. I had felt his spit on my face. [...] He raised his fist again. His eyes were wide. [...] He tried to hit me. He hurled a punch, full force, at my open face. If he had connected he would have broken my nose. Sqashed it flat. Crushed bones and shattered teeth. But somehow I managed to jerk my head away and down and the punch hit me on the shoulder. I heard, distinctly, the knuckle bones in his fist crunch and break as the force of the blow spun me round and right off my feet. [...] Mallabar was some distance off, scrabbling in the underground looking for something [...] He stood up, he had a stick in his left hand. He ran over towards me. [...] He hit me across the back. (206 f.)

Hope fürchtet um ihr Leben und rennt davon. Sie nutzt die Versorgungsfahrt ihres Kollegen Ian in die nächste Stadt zur Flucht aus dem Camp. Auf der Fahrt wird an Ian, der in den vergangenen Monaten oder Jahren die ,Täter-Gruppe` der Schimpansen, die ,northerners', beobachtet, aber von den Attacken nichts bemerkt hatte, das affektive Ausmaß einer solchen epistemologischen Umwälzung, wie sie Mallabar erfahren haben muss, noch einmal als unmittelbare Reaktion auf Hopes Erzählung nachvollzogen: „,Good Christ', Ian said, with a tone of shocked awe in his voice. ,Good God Almighty.' [...] Ian exhaled. ,Ooh God', he said, worriedly. ,Ooooh God.““ (209). „[S]tunned, sandbagged“ konstatiert Ian: „There’s too much to take in. I keep thinking of other things. Jesus. [...] The chimps. Pretty earth-shattering“ (209). „, [T]errible shock“, „,shocked awe“, „too much to take in“, „earth-shattering“: Das Beobachtete und das Begreifen des Beobachteten sind hier eine gravierende affektive Erfahrung.

Es gibt im Roman nach dem tätlichen Angriff im Wald keinen Kontakt mehr zwischen Mallabar und Hope. Mallabars Frau Ginga tritt als Vermittlerin auf, die der Erkenntnis-Katalystin Hope kündigt und dafür sorgt, dass es Hope mit der erzwungenen Auflösung ihres Forschungsvertrages nicht mehr erlaubt ist, ihre Ergebnisse selbst zu publizieren. Mallabar eignet sich also das neue Wissen umfänglich an und scheint sich von dieser Erfahrung zumindest insofern zu erholen, als er Hopes Beobachtungen und Schlüsse in sein fast fertiges neues Buch einarbeitet (vgl. 294). Im Sprechen der anderen Figuren über seinen affektiven Zustand zeigen sich jedoch die höflichen Formeln für den Nervenzusammenbruch: „Not very well. [...] something of a, a nervous collapse“ (298, Hauser); ,,[h]e went mad“ (292, Hope), ,[h]e's not well [....] [.] Very depressed. It's difficult“ (293, Ginga); „I wondered seriously, for the first time, about the true extent of Mallabar's nervous exhaustion“" (294, Hope).

Wie Mallabar wird auch Hopes Ehemann John Clearwater von seinen Emotionen im Erkenntnisprozess überwältigt. Während Mallabar durch die Einsicht in die Schimpansengewalt die Beherrschung so weit verliert, dass er nervös kollabiert, ist John Clearwaters Emotionalität im Roman explizit eine Verbindung 
seiner manisch-depressiven Disposition mit der Forschungsarbeit in der theoretischen Mathematik. John ist dort am besten gelaunt (,,almost in a mood of quiet self-congratulation“, 193), wo seine Manie in Bezug auf seine Forschungsüberlegungen freien Lauf hat, und dort am stärksten von den Gemütsschwankungen in Mitleidenschaft gezogen, wo diese Spekulationen ins Leere laufen oder gar von jemand anderem gleichzeitig gedacht werden. ${ }^{340}$ Dabei wird von Boyd via Hopes Beobachtungen herausgestellt, dass Johns mathematische Art und Weise zu denken und seine affektive psychische Erkrankung nicht mehr zu trennen sind. Eingeführt wird John mit der Zusammenfassung: „John Clearwater was a mathematician. It seemed an innocuous statement to make but, as far as Hope was concerned, that was both the root cause of his allure for her and the source of all his enormous problems“ (29). Forschungsthemen sind ihm Leidenschaften, gar Liebesobjekte: ,Turbulence is John Clearwater's new passion. Hope knows that his old passion, his old love for many years, was Game Theory“ (32). ,The victim, the catalyst, the guilty party“ seiner Persönlichkeitsveränderung, die Hope zunächst ,,subtly, indubitably“ (47) bemerkt, ist „,his work“: „,[H]er rival was mathematics“ (48). Wenn Hope feststellt, dass John fundamental , anders“ als sie ist (,the gap between them was not intellectual so much as conceptual - his brain operated on a different level and in a different sphere from hers", 48), betrifft dies sein spezifisch mathematisches Denken ebenso wie die zugrunde liegende Gehirnchemie. Der Preis seiner Genialität sind enorme und letztlich tödliche Gefühlsschwankungen:

John had knowledge that was denied to virtually everyone on earth. [...] He was at home in a world that was banned to all but a handful of initiates. You gained entrance if you possessed the necessary knowledge, but she knew that it was knowledge that was impossible for her to acquire. That was what made it special. It was magic in a way. [...] If you don't have the right kind of brain then all the effort and study in the world can't help you. [...] To enter the secret mathematical world John Clearwater inhabited, you had to have a rare and special gift: a particular way of thinking, a particular cast of mind. (53)

Dazu gehören auch jene Kopplungen von körperlichen Tätigkeiten und intellektueller Arbeit: Um eine geeignete Denkweise (wieder-)zu finden, gräbt John manisch tiefe Löcher, denn bei seiner Arbeit an Turbulenz verändert sich sein Denken von dem eines ,figure man“ zu ,seeing answers, solutions, in shapes“ (142, Hervorheb. im Original). Diese Denkweise in Formen droht ihm immer wieder zu entgleiten und so ,the digging he had done - for some inexplicable reason - had rejuvenated the imaging power": „Just the exertion, maybe. The physical effort.

\footnotetext{
${ }^{340}$ Der Roman bringt hier gleich an zwei Stellen, in zwei unterschiedlichen Fachrichtungen das Problem auf, wie affektiv damit umzugehen sei, dass die eigene Forschungsarbeit jemand anderem Erfolg einbringt: Nicht nur verwendet Mallabar Hopes Ergebnisse und Beobachtungsnotizen, um das Narrativ von der Entdeckung der Schimpansengewalt selbst zu erstellen und zu beherrschen und seine eigene Forschungslinie zu revolutionieren; John muss einsehen, dass seine Überlegungen, „The Clearwater Set“ ( $B B$, S. 275), bis zur Formel genau zeitgleich von jemand anderem gedacht wurden (,„Unreal', he said. ,Dazzling. But someone else thought it up.“”, $B B$, S. 276).
} 
[....] Or maybe just the fact that I was cutting a shape in the ground. A square, a rectangle“ (142). Wenn diese Gehirnformation bei John affektiv zuschlägt, in der Erkenntnis vom Mangel an Erkenntnismöglichkeiten, dann auf fundamentale Weise, wie die oben zitierte Passage seines Nervenzusammenbruchs zeigt.

Im Gegensatz zu diesen beiden Figuren, an denen der Zusammenhang von Emotionalität und Forschungsarbeit die Implikation der beklemmenden Aufstauung und des katastrophalen Ausbruchs mit sich führt, wird Hope Clearwater von Beginn ihrer Erzählung an als affektiv robuste und kontrollierte Persönlichkeit dargestellt. Sie selbst führt sich mit einer Exposition in ihre Erzählung von der Feldforschung ein, die sogleich ihr affektives Verhältnis zu ihrem Forschungsgegenstand bestimmt:

I never really warmed to Clovis, he was far too stupid to inspire real affection, but he always claimed a corner of my heart, largely - I suppose - because of the way he instinctively and unconsciously cupped his genitals whenever he was alarmed or nervous. It was rather endearing, I thought, and it showed a natural vulnerability, in strong contrast to his usual moods: raffish arrogance or total and single-minded self-absorption. (3)

Die Boyd von Griem diagnostizierte Unsentimentalität gegenüber den Affen zeigt sich hier wohl am deutlichsten: Hope ist durchaus disponiert, ein affektives Verhältnis zu ihren Forschungstieren zu unterhalten, kennt diese „as well as I knew my family“ (20). Dies weitet sich jedoch nicht zu einem unkritischen allumfassenden Gefühl für die Art aus, wie es Mallabar unterhält. Tiere sind durch ihre jeweiligen Eigenarten liebenswert (,endearing“), werden aber als Individuen auch aufgrund ihrer eigenen emotionalen Kennung und ihrer Persönlichkeitszüge (,raffish arrogance or total and single-minded self-absorption“) wahrgenommen.

Dem Roman ist das sokratische Motto „An unexamined life is not worth living“" (o. S.) vorangesetzt. Dieses Motto ist ebenso kennzeichnend für die ethologische Praxis der ,examined life stories ' der Affen wie für Hopes Selbst-Verhältnis. In Brazzaville Beach zeichnet sich die Protagonistin, wie Griem bereits festgestellt hat, dadurch aus, dass sie ihren analytischen Blick vor allem auch auf sich selbst wirft. Dies betrifft auch ihr Gefühlserleben: „I considered myself; analysed how I felt" (221). Durch die unterschiedlichen Ebenen und Formen der Erzählung hindurch lässt sich aus Boyds Figurenzeichnung und Hopes Selbstauskunft eine Wissenschaftlerinnenfigur herausarbeiten, die überwiegend durch eine nüchterne aber im Rahmen des Romans bestimmt positiv evaluierte Forschungsemotionalität charakterisiert ist und die vor allem Vertrauen in die eigenen Fähigkeiten und Freude an der wissenschaftlichen Arbeit hat. Als Hope die Arbeit als botanische Gutachterin übernimmt, stellt sie fest, dass

[s] he had forgotten this facet of her personality: the dogged application, and exultation in, her expertise. This was what she had trained herself to do; this was why she was educated. Problems were presented to her and she found a way to solve them. [...] [S]he enjoyed and savoured the unrelenting rigour of her approach to her task, the unswerving persistence of her routine and the evident success of her experimentation. In her work 
she was achieving something irrefutably concrete. However recondite, however parochial, she was adding a few grains of sand to that vast hill that was the sum of human knowledge. $(104 \mathrm{f} .)^{341}$

Hope besitzt „a quiet but strong pride in her abilities“: „latent self-confidence never far below the surface“ (105). Wenn sie arbeitet, ist sie „,calm and full of her task and all her senses were stimulated“ (106). Diese Versenkung kann durch erregte Gefühle abgelöst werden, etwa wenn sie etwas Neues erkennt, doch diese unterliegen ihrer Selbstbeherrschung: Als sie erstmals Kannibalismus unter den Schimpansen vermutet, heißt es: „I tried to keep my thoughts calm and rational. [...] I checked my natural excitement: softly, softly, I thought“" (23). Auch wird Hope nicht vom Grauen oder Ekel überkommen, als sie die Schimpansengewalt beobachtet, wie sie retrospektiv herausarbeitet: „She can admit to herself now that [...] [a]longside her alarm and her shock had been another sensation: excitement. She felt lucky, almost blessed. It was Hope Clearwater who was witnessing these extraordinary events" (189). Eine ganz besondere, komplexe Forschungsemotion wird hier in einer reflektierenden Passage unter dem Titel „FAME“ zusammengefasst: Des Schrecklichen erstmals gewahr zu werden, bedeutet, als erste Zeugin Erkenntnis zu generieren, Ruhm zu erlangen, der wissenschaftliche Eitelkeit auf eine unpräzidierte Art Genüge zu tun.

Hope hat im Feld jedoch vorwiegend ein Regime ausgearbeitet, um ihre Gefühle ,in check` zu halten:

I lay down on my camp bed, closed my eyes and, as always when I returned home at the end of the day, tried not to let my feelings overwhelm me. I arranged my day and my routine in such a manner as not to leave myself with much time alone and little to do, but this moment of the early evening, [...] always brought in its train a familiar melancholy and cafard and, in my particular case, an awful self-pity. I forced myelf to sit up, took some deep breaths, inveighed powerfully against the name of John Clearwater, and went to sit at the little trestle desk where I worked. There, I poured myself a glass of scotch whiskey and wrote up my field notes. (7, Hervorheb. im Original)

Wo sie sich tatsächlich von ihren Gefühlen eingenommen fühlt, verläuft Hopes Bestandsaufnahme ihrer Affektivität ungewöhnlich prozesshaft: „I sat on my bed and allowed my swiftly alternating moods to dominate me, unchecked. I felt by turns apathetic, sullen, hard-done-by, frustrated, baffled, hurt and, finally, contemptuous and independent" (292). Hier liegt die Emphase bezeichnenderweise darauf, dass dieser Prozess sie nicht überkommt, sondern sie ihn zulässt (,allowed my $[\ldots]$ moods to dominate me"), weil er dialektisch auf seine eigene Auflösung zuläuft.

\footnotetext{
${ }^{341}$ Hope grenzt diese Feststellung über eine vergessene, geschätzte Selbstqualität von einem ,privaten“ Bereich ab: „It was a feature of her character which, when it was not required or employed, she somehow forgot. It did not feature in her private conception of herself. The fanciful, wishful version of Hope Clearwater tended to downplay the professional scientist in her" (BB, S. 104).
} 
Erst als Hope aus dem Forschungskontext gefallen ist und uns aus dem Strandhaus heraus ihre Geschichte(n) erzählt, scheint dieses affektive Muster der beherrschten und examinierten Emotionalität sich zugunsten einer Art Gleichmütigkeit umzuformen. Doch dies ist deutlich ein Zwischenstadium, eine ,Auszeit", für Hope: „I am here because two sets of strange and extraordinary events happened to me", heißt es im Prolog, ,,and I needed some time to weigh them up, evaluate them. I have to make sense of what has taken place, before I can restart my life in the world, as it were. Do you know that feeling? The urge to call a temporary halt, to say: enough, slow down, give me a break" (xiii). Das Ende der Analyse braucht eine Zwischenzeit, einen temporären Raum des Abwägens und Evaluierens, bevor eine Handlungsimplikation aus dem Gefühl entstehen kann. Bis dahin hat sie „taken new comfort and refuge in the doctrine that advises one not to seek tranquillity in certainty, but in permanently suspended judgement", „enjoying my indecision, my moral limbo“(313).

\subsection{5 „In science, so in life ${ }^{\mu 342}$ : Emotionsphilosophie}

Hopes analytischer Blick ist nicht nur ethologisch grundiert, sondern fast schon anatomisch sezierend darin, wie er Emotionalität daraufhin befragt, was sie ist, und wozu sie dient. Dabei arbeitet der Roman an einem Emotionsverständnis, in dem Emotionen als viszeral spürbare Empfindungen und zugleich als Phänomene erscheinen, die mithilfe konzeptueller Anleihen begriffen werden können.

\subsubsection{Die Logik der Affekte:,gut feelings'}

In Ians Reaktion auf Hopes Bericht von der Gewalt der Schimpansen und Mallabars gewaltvoller Reaktion darauf - betäubt (,stunned“) und niedergeschlagen (,sandbagged") - scheint es bereits auf, ebenso wie in Johns Gefühlsausbruch, seinem konvulsiven Weinanfall mit dem Würgen, Grunzen, Heulen, Ausstoßen: Für Boyds Erzählerin erschließen sich emotionale Phänomene über ihren somatischen Ausdruck: „I had the sensation of a sagging, a falling inside me [...] [,] I felt tears smart my eyes“ (44), heißt es angesichts der Erleichterung, dass die Schimpansin Lena mit ihrem Baby gesichtet werden konnte. Als sie den verwesenden Körper von Muffin findet, „I gagged and felt saliva swirl into my mouth. I felt faint and shocked" (181). Auf die Nachricht hin, dass ihr Liebhaber Usman Shoukry nicht von seiner letzten Flugmission zurückgekehrt sei, „I blushed. My eyeballs felt hot in their sockets“" (281). Als der Rebellen-Anführer Dr. Almicar vor ihren Augen von einer Kugel tödlich getroffen wird, fühlt sich Hope dagegen ,lightheaded, absurdly fit, as if I were being blown along by a stiff, following breeze“, "quivering with huge reserves of energy suddenly unleashed, as if there had been hidden stockpiles of adrenalin in my body, now bequesthed me to do whatever I wanted“ (268). Wut lässt sie „almost tearful with frustrated anger“ (285) werden

${ }^{342}$ BB, S. 49. 
oder sie fühlt ,her anger pinch her nostrils“ (285); sie ist ,rigid with disappointed anger" oder ,[s]till trembling, positively vibrating with anger at him“ (303). Angesichts der Enthüllung, dass Mallabar die Entdeckung der Schimpansenkriege als die seine ausgibt, heißt es: „I felt a tightening in my head, as if a belt were being cinched around my skull. [...] A sense of frustration was building inside me that was making my shoulders hunch and my scalp crawl“" (291). Als sie realisiert, dass ihre Forschungsergebnisse nicht wird publizieren können, dass der „FAME“ ihr versagt bleibt, heißt es: „I felt an associated amusement - an oddly tearful, resigned amusement - shake my body in a weak chuckle“" (294). Besonders bemerkenswert sind die Empfindungen, die als , gut feelings“ umschrieben werden: ,, an odd subsidence in my gut, a hollow feeling that made me want to laugh and cry and the same time“ (225), oder ,a burning, like indigestion, in my oesophagus“, ,a cold agitation“ (199); ,an agreeable shifting in her gut, an excited tightening of her sphincter" (110).

In Hopes selbstreflexivem Beobachtungsverfahren wird immer zunächst die körperliche Symptomatik (,the symptoms“, 225) eines Fühlens registriert. Dann wird diese Symptomatik hinsichtlich des zugehörigen affektiven Phänomens analysiert - es heißt wörtlich ,[a]nalysing the feeling further“ (86) -, um eine Bedeutung herzustellen: Das ,excited tightening of her sphincter" bedeutet ihr „,she was glad to be back [in the cottage in Knap]“ (110); ,, a distinct and unusual sensation of pleasure“, dass sie ,[was] actually looking forward to getting back to work“ (86). Das „burning [...] in my oesophagus“ jedoch ,was the physical correlative of a crucial indecision, a growing panic of inertia“ (199).

Doch diese ,gut feelings“ verweisen nicht schlicht auf einen sich im Viszeralen manifestierenden biologischen Instinktanteil menschlicher Emotionalität. Sie besitzen einen epistemologischen Gehalt, der aus dem Prozess der Bedeutungsproduktion entsteht. Boyd erinnert mit Hopes affektiver Introspektion an Teresa Brennans Betonung einer ,logic of the flesh': Für Brennan sind die Empfindungen registrierenden Sinne ,vehicles of attention“, welche die ,cognitive faculty of linguistic thought with the fleshly knowledge of codes of the body“ 343 verbinden, nicht als Emotionen selbst, sondern als ,vehicles for their discernment". ${ }^{344}$ Brennan entwickelt in ihrer Beschäftigung mit der Affektübertragung eine Vorstellung von Verbildlichung als Medium der Verknüpfung von körperlichen Empfindungen und sprachlichem Bewusstsein: ,[T]he gateway between (linguistic) consciousness and the codes of bodily sensation is manned by visual images. That is to say, to make itself conscious, a

\footnotetext{
${ }^{343}$ Brennan: Transmission of Affect, S. 136. Vor psychoanalytischem, emotionshistorischem und sozialpsychologischem Hintergrund beschäftigt sich Brennan mit der Affektübertragung als so sozialem wie biologisch-physischem Prozess: „The origin of transmitted affects is social in that these affects do not only arise within a particular person but also come [...] via an interaction with other people and an environment. But they have a physiological impact"; mit den körperlichen Veränderungen, die eine Affizierung auslöst, verändert ,the transmission of affect [...] the biochemistry and neurology of the subject" (S. 3).
}

${ }^{344}$ Brennan: Transmission of Affect, S. 137. 
bodily process has to be imagined - given an image." ${ }^{345}$ Boyds Roman liefert uns ein solches bildgebendes Verfahren, Emotionalität zu fassen und bewusst zu machen. Sei es die sich wie ein strömendes, schweres Gas um die Figuren windende Traurigkeit oder die schalartig umhüllende Verlegenheit: Erst in der bildlichen Hervorbringung wird das körperliche Befinden begreif- und deutbar. Dies ist es, was Brennan unter Einsicht (,discernment ${ }^{\circ}$ ) versteht: Gefühle sind ihr Empfindungen, ,that have found a match in words". 346 Daher sind sie auch „sensory states produced by thought“":347 „Feelings are meant to be information about whether a state is pleasurable or painful, whether one is attracted to something or averse to it." 348

Gerade Hopes Analyseverfahren macht diese Einsicht in einem dreiteiligen Prozess deutlich: Die körperliche, sinnliche, organische Empfindung wird sprachlich ausgedrückt, sodass ein Bild entsteht. Anhand dieses Bildes kann auf einen affektiven Zustand geschlossen und aus diesem wiederum dann letztlich eine Handlungsimplikation abgeleitet werden. Die „odd subsidence in my gut“ ist ein „hollow feeling that made me want to laugh and cry at the same time" und indiziert ihr, dass sie sich auf die absurde Situation, inmitten der Endphase eines Bürgerkrieges von einem jugendlichen Volleyball-Team namens ,Atomique Boom ' entführt worden zu sein, einlassen kann. Das „burning feeling“ dagegen ist das physische Korrelat „of a crucial indecision“ und betrifft das Verhalten vis-à-vis der Schimpansenattacke: „What should I do here?“ (199). Das „excited tightening in her sphincter" bedeutet nicht nur die Freude, wieder zurück in ihrem Arbeitsbereich in Knap zu sein, sondern führt auch zu einer Reflektion über den Stand ihrer Ehe und die Einsicht, dass diese ein Fehler gewesen sein mag (110 f.). Emotionalität ist in Brazzaville Beach somit nicht nur an eine somatische Indikatorfunktion (Brennans ,logic of the flesh') gebunden, sondern Emotionen werden auf Basis des körperlichen Empfindens erschlossen, decodiert und handlungsrelevant gedeutet.

\footnotetext{
${ }^{345}$ Brennan: Transmission of Affect, S. 150, Hervorheb. MS.

${ }^{346}$ Brennan: Transmission of Affect, S. 116.

${ }^{347}$ Brennan: Transmission of Affect, S. 116.

${ }^{348}$ Brennan: Transmission of Affect, S. 116. Diese kantische Position ist für Brennan auch ,the classic and only basis for distinguishing feelings and affects" (S. 116). Für Brennans Ansatz, in dem Affekte verglichen mit den Gefühlen ,thoughtless' sind, ist nicht nur ihre Teilhabe an einer postmodernistischen Abgrenzung von der ,expressive hypothesis' wichtig, in der, wie Rei Terada hervorhebt, „expression is the dominant trope of thought about emotion“ Terada, Rei: Feeling in Theory. Emotion after the „Death of the Subject“. Cambridge, Mass./London 2001, S. 11. Brennan setzt gegen das Affekt-Verständnis der James-Lange-Theorie, ,that bodily responses give rise to affective states“, ihr Verständnis von Affekt als ,the physiological shift accompanying a judgment". Brennan: Transmission of Affect, S. 4 f. Wesentlich ist auch Brennans Verwurzelung in psychoanalytischer Theorie, vor allem der Vorstellung einer kathartischen Gesprächstherapie: „Language releases us from the affects (through genuine psychoanalysis or other practices of discernment) via words that express something occluded and thereby releases the energy deployed in the occlusion" (S. 140).
} 


\subsubsection{Katastrophentheorie der Emotionalität}

In Brazzaville Beach wird zudem Emotionalität mittels Anleihen aus dem Bereich mathematisch-physikalischen Denkens erschlossen. Dies beginnt bei der Frage danach, ob Emotionalität „NOISE OR SIGNAL?“ (44) sei. In der so betitelten, kursiv gesetzten reflexiven Passage geht es vordergründig um das Problem, bei Geräuschen - z. B. als Schwerhöriger - ,noise from signal“ (44) zu unterscheiden. Hope sieht dies als allgegenwärtiges Problem:

I think: join the club. Learning to listen is like any process of education. You have to sift through a mass of phenomena and discard what is unimportant. You have to distinguish the signal from the noise. When you find the signals a pattern might emerge, and so on. (44)

Hopes Bemerkungen lassen sich jedoch auch als Ergebnis einer ,éducation sentimentale ' deuten, die es ihr ermöglicht zu unterscheiden, welche affektiven Phänomene als Begleiterscheinungen ,Geräusch' und welche als Gefühle ,Signal' sind: welche einfach durchlebt, und welche als handlungsweisend befolgt werden müssen. Dass dies keine einfache Aufgabe ist, zeigt ihr Verweis auf Johns neues Augenmerk für Varianz, für „systems [...] erratic and discontinuous“ (45). Wie wir fühlen, ist bedingt von der „,unruly world we live in“, von der „happenstance“ (45), die uns ereilt. ${ }^{349}$ An anderer Stelle erinnert sich Hope daran, wie ihr John von seinen Überlegungen zu turbulenten Strömungen berichtete, und wie ,people tried to understand turbulence by writing endless and ever more complicated differential equations for the flow of fluids. As the equations became more involved and detailed, so their connection to the basic phenomenon grew more tenuous" (55). John dagegen nähert sich der Sache durch Formen: „He decided to look at the shapes of turbulence and, immediately, he began to understand it“ (55). Unter dem Titel „DIVERGENCE SYNDROMS“ geht es hier um „forms of erratic behaviour“: „,[S]omething you expect to be positive will turn out to be negative. Something you assume will be constant, becomes finite. Something you take confidently for granted, suddenly vanishes" (55). Betrachtet man den Roman durch die Linse seines Emotionsdiskurses, lassen sich diese Ausführungen auf Emotionstheorien ausweiten, die durch ihre abstrahierende Modellbildung das Phänomen selbst aus dem Blick zu verlieren drohen. Boyd schlägt vor, durch eine Konzentration auf die zugrunde liegenden Formen, die Emotionalität auf basaler, somatischer Ebene einnimmt, das Ganze zu verstehen - und zwar als immer potenziell erratisches System. So kehrt auch an der oben zitierten Stelle der Affekt selbst in Johns Erläuterungen ein:

This sort of erratic behaviour terrifies mathematicians, John said [...]. But people were learning, now, that the key response to a divergence syndrome was not to be startled, or confounded, but to attempt to explain it through a new method of thought. Then, often, what seemed at first shocking, or bizarre, can become quite acceptable. (55)

\footnotetext{
${ }^{349}$ Auch hier nimmt Boyds Fiktion wieder Teresa Brennans Konzeption von Affektivität vorweg, die gegenüber der Vorstellung vom autonomen Individuum und seinem ,affective self-containment“ neben der sozialen Komponente vor allem das weiter gefasste, ,environment“ als Interaktions- und Affektationsquelle betont. Brennan: Transmission of Affect, S. 2 ff.
} 
Dieser Hinweis scheint im Kontext des Romans allgemeingültig ausweitbar auf einen Umgang mit erschreckenden Phänomenen oder irritierenden Herausforderungen, wenn auch, wie Hope anmerkt, nicht ,so easy to apply in a moment of crisis“" (55).

In einer weiteren reflektierenden Passage geht es später um Katastrophentheorie, ,the study of abrupt change, the catalogue of discontinuity“ (282). Unter den ,seven types of catastrophe“ (282) interessieren Hope die Faltungs-Katastrophe (,fold catastrophe“) und die Spitzen-Katastrophe (,cusp catastrophe“). Erstere ist ,the most simple paradigm of change. The balloon bursts, the catastrophe has occured. There is no going back“ (282). Für Hope ist dies die irreversible chronologische Struktur des Lebens: „Fold catastrophes cannot be reversed“ (282). Aber:

Cusp catastrophes are different. In a cusp catastrophe there is always the chance of recovery, a possibility of return to the pre-catastrophic state. Being knocked unconscious would qualify as a cusp catastrophe, so would a nervous breakdown or an epileptic fit, or a boiling cattle of water. (282)

Wie Hopes Beispiele zeigen, bietet sich die Kategorie der Spitzen-Katastrophe an, um Gefühlsausbrüche, wie jenen ,nervous breakdown“ Johns zu fassen. ${ }^{350}$ Die epistemologische affektive Erfahrung, die Mallabar mit den Schimpansen macht, dürfte dagegen eine Faltungs-Katastrophe sein, eine Erfahrung, die keinerlei Rückkehr zum vorkatastrophischen Zustand verspricht, und ihn unwiederbringlich verändert hat.

Boyds Roman greift hier nicht nur mathematische Theoriebildung und ihre Verwendung in der Verhaltensforschung auf, sondern scheint auch an spezifisch postmoderner Emotionsphilosophie zu partizipieren: In seiner Beschäftigung mit der ,Autonomy of Affect‘ sieht Brian Massumi Affekt oder ,intensity“ als ,akin to what is called a critical point, or a bifurcation point, or a singular point, in chaos theory and the theory of dissipative structures".351 Massumi sieht in der Folge Baruch Spinozas Affekt und Emotion als unterscheidbare Phänomene eines Prozesses: ,[E]motion and affect [...] follow different logics and pertain to different order“. ${ }^{352}$ Affekt ist „unqualified“, „,not ownable or recognizable“, 353 eine ,,affection of (in other words an impingement upon) the body, and at the same time the

\footnotetext{
${ }^{350}$ Die Katastrophentheorie selbst bietet phänomenologische Modelle an, die in der Verhaltensforschung Anwendung finden, z. B. bei der Berechnung von affektbedingtem Aggressionsverhalten; vgl. Zeeman, Eric C.: Catastrophe Theory. In: Scientific American (April 1976), S. 65-70, 75-83. Interessanterweise heißt es bei Zeeman: „Another human behaviour that can be described by the cusp model is self-pity and the catharsis that sometimes relieves it" (S. 69). Dabei geht es um im Selbstmitleid angestaute Frustration, die sich in einem kataklysmischen Moment freisetzt, dem der Übergang in einem ausgeglicheneren emotionalen Zustand folgt.
}

${ }^{351}$ Massumi: The Autonomy of Affect, S. 93.

${ }^{352}$ Massumi: The Autonomy of Affect, S. 88.

${ }^{353}$ Massumi: The Autonomy of Affect, S. 88. 
idea of the affection" ${ }^{354}$ Erst wenn sich eine Idee der Idee der Affektation bildet, setzt bewusste Reflexion ein, d. h. bildet sich das Phänomen zur Emotion um:

An emotion is a subjective content, the socio-lingustic fixing of the quality of an experience which is from that point onward defined as personal. Emotion is qualified intensity, the conventional, consensual point of insertion of intensity into semantically and semiotically formed progressions, into narrativizable action-reaction circuits, into function and meaning. It is intensity owned and recognized. ${ }^{355}$

Obwohl sie hinsichtlich der Affekt/Emotions-Unterscheidung unterschiedlich argumentieren, finden Brennan und Massumi in einer Logik des Somatischen wieder zusammen. Obwohl Massumi bei seiner Beschreibung auf der Hautoberfläche verbleibt (affektive Intensität des Gefühls ,,is embodied in purely autonomic reactions mostly directly manifested in the skin - at the surface of the body, at its interface with things “356), ähnelt Hopes selbstanalytische Verfahrensweise auch dieser Differenzierung und zugleich Prozessualisierung von Affekt und/zu Emotion: von der zutiefst körperlichen Empfindung des ,Zusammenstoßes“ (bei Massumi: ,impingement") von Welt und Bewusstsein, wie sie für Hope vor allem in der Tiefe des Bauches und am Kopf vollzogen wird, zu einer Semantisierung und Semiotisierung dieser Erfahrung von Intensität als interpretier- und übersetzbares emotionales Phänomen - verbunden mit der Handlungsimplikation, die aus den narrativierbaren , action-reaction circuits' erwächst. ${ }^{357}$

Johns mathematische Leidenschaft wechselt im Roman schließlich von der Turbulenz zur Topologie, weil ihn hier interessiert, ,what remained constant in an object, regardless of the force or scale of its transformation. When something is bent, stretched, or twisted, he said, certain features of it resist deformation. He wanted to investigate these unchanging features“ (286 f.). Ob als „permanence“

\footnotetext{
${ }^{354}$ Massumi: The Autonomy of Affect, S. 91, Hervorheb. im Original.

${ }^{355}$ Massumi: The Autonomy of Affect, S. 88.

${ }^{356}$ Massumi: The Autonomy of Affect, S. 85.

${ }^{357}$ Hopes Selbstanalyse auf dem Gebiet ihres affektiven Erlebens unterstreicht die Bedeutung von Selbstreflexivität, wie sie Patricia Ticineto Clough im ,Affective Turn“ in einer Linie mit Deleuze und Guattari, Spinoza und Bergson verortet. Affekt wird hier konzeptualisiert als „bodily capacities to affect and be affected or the augmentation or diminution of a body's capacity to act, to engage, and to connect, such that autoaffection is linked to the self-feeling of being alive“. Clough betont - vermeintlich in Abgrenzung von Massumi - die „nonlinear complexity out of which the narration of conscious states such as emotion are substracted", denn sie sieht einen ,reflux back from conscious experience to affect, which is registered, however, as affect“. Clough, Patricia Ticineto: Introduction. In: Dies./Jean Halley: The Affective Turn. Theorizing the Social. Durham/London 2007, S. 1-33, hier S. 2. So setzt sie eine Konservierung und Wiederholung von Aktionen und Kontexten an, die jedoch nie bewusst werden kann. Sianne Ngai sieht die Aufgabe von Emotionen mit Rei Terada in ihrer Funktion als „,interpretations of predicaments“. Ngai: Ugly Feelings, S. 3. Massumis, Teradas, Ngais und auch Cloughs Überlegungen zeigen, wie die postmoderne Emotionsphilosophie somit aus einer derartigen neumaterialistischen Konzeptionalisierung des Affektbegriffs ein kritisches theoretisches Potenzial schöpft. Vgl. dazu auch kritisch: Plamper: Geschichte und Gefühl, S. 274.
} 
(286), ,constancy“ (287) oder - in Johns Wortprägung - „topological invariants“ (287), es geht um ,things that endure [...] even though everything else about them is changing" (287). Auch hier lässt sich wieder, mit Blick auf die Geschichte(n), die uns Hope berichtet, etwas Anderes als nur das mathematische Thema ins Auge fassen: Ist nicht Hopes Erzählung eine Erforschung dessen, was sich verändert hat und dessen, was durch all die Erzählebenen hindurch konstant geblieben ist? Wenn ja, wäre dies nicht genau jenes analytisch-emotionale Verfahren, das sie immer wieder auf ihre Empfindungen und Gefühle als Indikatoren verweisen lässt? Einen letzten Hinweis hierzu gibt es unter dem Titel „FINESSE“, wenn es um Blaise Pascals Verteidigung des Calculus geht:

\footnotetext{
The formal demands of logic, he said, cannot always have the last word. [...] It seemed right, even if it could not be fully or pedantically justified [...]. On this sort of occasion, Pascal said, your intuition rates higher than rigorous proof. Rely on your heart to tell you if this is the right mathematical step to take. In cases like these the correct mental attitude to apply to the task in hand was one of ,finesse " rather than ,logic', finesse being employed here in its original sense, meaning, delicacy of discrimination ‘. (300 f.)
}

Boyds Emotionsbegriffsbildung entwirft in diesem Sinne eine epistemologische Emotionalität als Äquivalent der ,Finesse', eine Nutzbarmachung des Emotionalen als ergänzendes Unterscheidungs- und daher Erkenntnismedium und Handlungsinstrument. Hope ist die Vertreterin dieser ,mental attitude', wie sie freimütig eingesteht: „I like the idea of finessing my way to a correct answer, rather than relying on the power of logical argument. Perhaps I shall finesse my way through the rest of my life?" (301).

\subsubsection{Fiktion als kathartischer Raum einer Rationalität des Gefühls}

In Brazzaville Beach spielt William Boyd durch, wie die Forschungsarbeit affizieren, welches Ausmaß in diesem Sinne epistemologisch relevante Emotionalität annehmen, wie sie im Gleichgewicht gehalten und unter Zuhilfenahme eines analytischen Verfahrens nutzbar gemacht werden kann. Brazzaville Beach thematisiert die körperliche Intensität des Fühlens, aber auch den intellektuellen Gehalt von Emotionalität - und kommt damit dem von Arbogast Schmitt umrissenen Emotionsbegriff Aristoteles', seiner „Kultur des Gefühls“358 nahe: Schmitt bescheinigt Aristoteles einen ,grundlegend anderen Begriff von Kognition [als er uns vertraut ist]“, der es ermögliche,

${ }^{358}$ Schmitt: Kommentar, S. 334. 
anstelle des Neben- und Gegeneinanders einer bewussten und einer emotionalen Intelligenz an der Vorstellung der Einheit einer Intelligenz im Menschen festzuhalten, aber einer Intelligenz, die in ganz unterschiedlicher Weise im Menschen wirksam ist, wenn er rational urteilt und bewertet und wenn er emotional denkt und bewertet. ${ }^{359}$

Wie in der postmodernen Affekttheorie sind auch in Schmitts Aristoteles-Kommentar Gefühle „Mischformen aus Erkenntnisakten, Lustgefühlen und Strebemomenten". ${ }^{360}$ Sie entstehen aus einem komplexen Zusammenwirken mehrerer psychischer Akte. Es ist gerade Schmitts Umriss der ,Rationalität der Gefühle“ bei Aristoteles, der für Boyds Roman und vor allem seine Protagonistin Hope wegweisend scheint:

Die mögliche Rationalität der Gefühle besteht nicht darin, dass wir sie bewusst erzeugen, sondern [...] darin, dass man angemessene Gefühle hat, d. h. eine Unterscheidungsfähigkeit für das, was ein Gefühl - etwa der Angst - erzeugt, und so z. B. fürchtet, was wirklich für einen eine Bedrohung ist, und dass man so fürchtet, wie es dieser Bedrohung angemessen ist, wann es angemessen ist, usw. ${ }^{361}$

Die den Gefühlen zugehörige Kognition entstehe nicht aus der nachträglichen Bewertung von Gefühlen, sondern aus der Art, wie ihre Auslöser aufgefasst werden. Dadurch lassen sich jene Gefühle erlangen, ,durch die man sich intellektuell und emotional in ein den jeweiligen, Gegenständen' gegenüber angemessenes Verhältnis bringt und ihnen dadurch auch handelnd optimal begegnen kann". ${ }^{362}$ Wie sehr es bei Aristoteles um das ,rechte $\mathrm{Ma} \beta^{`}$ gehe, zeige sich daran, dass sich die Rationalität der Gefühle darin finden lasse,

zu wissen und eine konkrete Erfahrung davon zu haben, was man fürchtet oder bemitleidet, in welchem psychischen Zustand man für diese Gefühle empfänglich ist, in welcher Weise, zu welcher Zeit, in welchem Ausmaß dieser Gefühle ihrem Gegenstand angemessen sind. ${ }^{363}$

In Hinblick auf Aristoteles' Katharsis-Verständnis, das sich besser an seiner Rhetorik und - wie Bernays deutlich gemacht hat - Politik nachvollziehen lässt als an der Poetik, aus der die Stammsätze zur Katharsis der Tragödie entlehnt sind, sieht Schmitt viel eher einen Fokus auf dem ,angemessene[n] Begreifen und Mitempfinden eines tragischen Handlungverlaufs“"364 liegen, als jenem „Vorgang, in

\footnotetext{
${ }^{359}$ Schmitt: Kommentar, S. 335. Siehe auch de Sousa, Ronald: Die Rationalität des Gefühls. Frankfurt a. M. 1997 (engl. 1987), S. 12 f.

${ }^{360}$ Schmitt: Kommentar, S. 339.

${ }^{361}$ Schmitt: Kommentar, S. 483.

${ }^{362}$ Schmitt: Kommentar, S. 483.

${ }^{363}$ Schmitt: Kommentar, S. 486. Besonders Aristoteles' Ausführungen zum Zorn in der Nikomachischen Ethik (IV.11.) machen dies deutlich. Vgl. Aristoteles: Nikomachische Ethik, S. 169.

${ }^{364}$ Schmitt: Kommentar, S. 478.
} 
dem die Tragödie ihr Werk auch für den Zuschauer erfüllt, d. h. es ist der Vorgang der Katharsis, in dem die Gefühle des Mitleids und der Furcht ,gereinigt ${ }^{*}$ werden“. 365 Mitleid und Furcht als ,die einer tragischen Handlung gemäßen Gefühle“366 entstehen, wenn ,,jemand ein tragisches Scheitern richtig begreift “ 367 nicht etwa, wie traditionell angenommen, durch Identifikation. Der Zuschauer hat ,an der Handlung nur durch Erkennen und Verstehen teil, er ist nicht selbst handelnd in sie verwickelt“. ${ }^{368}$ Für Schmitt ist Katharsis kultivierte Lust als direkter Ausdruck einer konkreten Erkenntnisweise:

Eine Tragödie mit Mitleid und Furcht Zu begleiten, heißt im Sinn der Aristotelischen Konzeption nicht, seine Fähigkeit, fremde Gefühle, Affekte, Leidenschaften mitzuempfinden, zu steigern, und auch nicht, sich gegen sie - mit der Kraft der Reflexion zu immunisieren oder sich von ihnen zu lösen, sondern: die ganz besonderen Gefahren, denen diese Handlung ausgesetzt ist, und die ganz besondere [sic] Gründe, warum diese Gefährdungen nicht genug beachtet worden sind, zu erkennen und das darin enthaltene Furchtbare und Mitleidverdienende mitzuempfinden. Der Zuschauer vieler Tragödien gewinnt auf diese Weise einen Erfahrungsschatz über die Gründe des Scheiterns menschlichen Handelns, und zwar einen im Gefühl selbst erlebten Erfahrungsschatz. ${ }^{369}$

Die Tragödie ist also für Aristoteles in Schmitts Kommentar ein Instrument, „den Erfahrungsschatz des ,Gefühlswissens “"370 zu erweitern. Als solches Instrument dient auch Boyds Roman Brazzaville Beach. Auf der Figurenebene lässt sich mithilfe der katastrophentheoretischen Konzeption von emotionalen Ausbrüchen eine kathartische Reinigung vollziehen - aber es muss dahingestellt bleiben, ob diese in jedem Fall zu einem dauerhaft ausgeglichenen Zustand führt. Mallabars Verschwinden als handelnde Figur im Rahmen der Erzählung ebenso wie Johns Suizid sind hier wohl als Indizien gegen die Möglichkeit der Harmonie zu werten. Kathartisch ist der Roman vor allem in jenem Sinn, als die Leserschaft an ihm und seiner narrativen Aufführung eines Spektrums an affektiven Phänomenen und ausgeübter Emotionalität ein rechtes $\mathrm{Maß}$ an Forschungsemotionalität erlernen kann. Der Roman bietet sich als Raum an, in dem anhand seiner Erzählperspektiven kathartisch verhandelt wird, unter welchen Voraussetzungen welche Emotionalität in welchem Maße in der Ethologie (und darüber hinaus) sinnvoll epistemologisch genutzt werden kann.

\footnotetext{
${ }^{365}$ Schmitt: Kommentar, S. 478, Hervorheb. im Original.

${ }^{366}$ Schmitt: Kommentar, S. 478.

${ }^{367}$ Schmitt: Kommentar, S. 478, Hervorheb. MS.

${ }^{368}$ Schmitt: Kommentar, S. 480.

${ }^{369}$ Schmitt: Kommentar, S. 500 f.

${ }^{370}$ Schmitt: Kommentar, S. 501.
} 
Open Access Dieses Kapitel wird unter der Creative Commons Namensnennung 4.0 International Lizenz (http://creativecommons.org/licenses/by/4.0/deed.de) veröffentlicht, welche die Nutzung, Vervielfältigung, Bearbeitung, Verbreitung und Wiedergabe in jeglichem Medium und Format erlaubt, sofern Sie den/die ursprünglichen Autor(en) und die Quelle ordnungsgemäß nennen, einen Link zur Creative Commons Lizenz beifügen und angeben, ob Änderungen vorgenommen wurden.

Die in diesem Kapitel enthaltenen Bilder und sonstiges Drittmaterial unterliegen ebenfalls der genannten Creative Commons Lizenz, sofern sich aus der Abbildungslegende nichts anderes ergibt. Sofern das betreffende Material nicht unter der genannten Creative Commons Lizenz steht und die betreffende Handlung nicht nach gesetzlichen Vorschriften erlaubt ist, ist für die oben aufgeführten Weiterverwendungen des Materials die Einwilligung des jeweiligen Rechteinhabers einzuholen. 